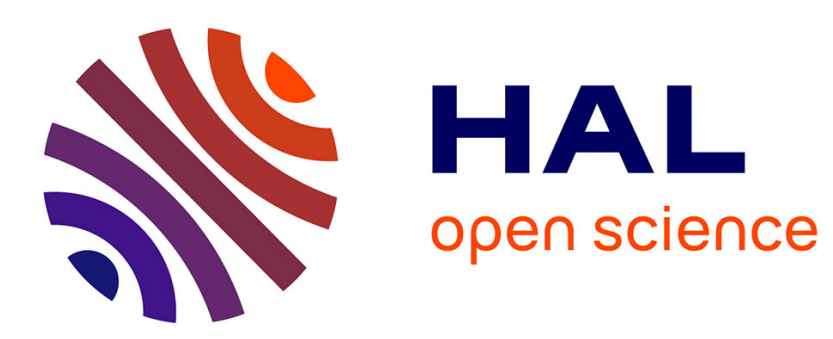

\title{
Prediction of user action in moving-target selection tasks
}

Juan Sebastian Casallas Suarez

\section{To cite this version:}

Juan Sebastian Casallas Suarez. Prediction of user action in moving-target selection tasks. Signal and Image processing. Ecole nationale supérieure d'arts et métiers - ENSAM; Iowa State University, 2015. English. NNT : 2015ENAM0018 . tel-01294975

HAL Id: tel-01294975

https://pastel.archives-ouvertes.fr/tel-01294975

Submitted on 30 Mar 2016

HAL is a multi-disciplinary open access archive for the deposit and dissemination of scientific research documents, whether they are published or not. The documents may come from teaching and research institutions in France or abroad, or from public or private research centers.
L'archive ouverte pluridisciplinaire HAL, est destinée au dépôt et à la diffusion de documents scientifiques de niveau recherche, publiés ou non, émanant des établissements d'enseignement et de recherche français ou étrangers, des laboratoires publics ou privés. 
École doctorale n ${ }^{\circ} 432$ : Science des Métiers de l'Ingénieur

\title{
Doctorat ParisTech
}

\section{THÈSE}

pour obtenir le grade de docteur délivré par

\section{l'École Nationale Supérieure d'Arts et Métiers \\ Spécialité "Informatique-traitement du signal"}

\author{
présentée et soutenue publiquement par
}

\section{Juan Sebastián Casallas}

le 26 juin 2015

Prediction of user action in moving-target selection tasks

Directeurs de thèse : Frédéric Mérienne et James Oliver

Co-encadrement de la thèse : Samir Garbaya

Jury

M. Jonathan W. Kelly, Associate Professor, Iowa State University

Président

M. Vincent Hugel, Professeur, Université de Toulon

Rapporteur

M. Samir Otmane, Professeur, Université d'Évry Val d'Essonne

Rapporteur

M. Michael C. Dorneich, Associate Professor, Iowa State University

M. Stephen B. Gilbert, Assistant Professor, Iowa State University

Examinateur

M. Jin Tian, Associate Professor, Iowa State University

Examinateur

M. Samir Garbaya, Maître de Conférences, Arts et Métiers ParisTech

Examinateur

M. Frédéric Mérienne, Professeur, Arts et Métiers ParisTech

Examinateur

M. James H. Oliver, University Professor, Iowa State University

Examinateur

Examinateur 
À Catherine.

Para Gaël Elías e Inés Sofía. 
... all models are approximations. Essentially all models are wrong, but some are useful. However, the approximate nature of the model must always be borne in mind. [Box and Draper 1987, p. 424] 


\section{TABLE OF CONTENTS}

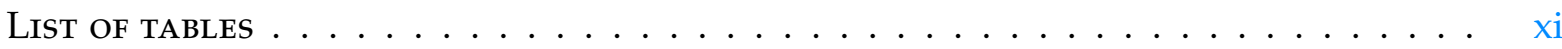

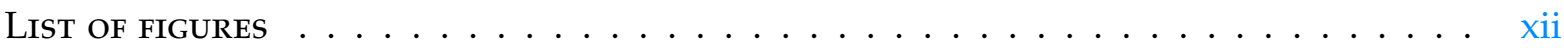

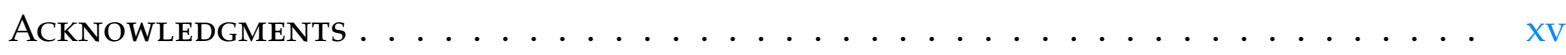

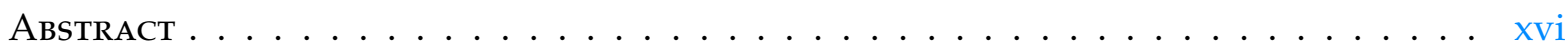

Chapter 1. Introduction $\ldots \ldots \ldots \ldots \ldots \ldots \ldots \ldots \ldots$

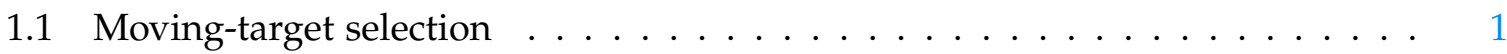

1.1.1 Moving-target selection challenges . . . . . . . . . . . . . 1

1.1.2 Moving-target selection enhancements . . . . . . . . . . . 2

1.1.3 Control strategies in moving-target selection . . . . . . . . . . . . 3

1.2 Research Layout . . . . . . . . . . . . . . . . . 5

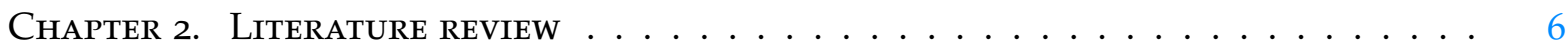

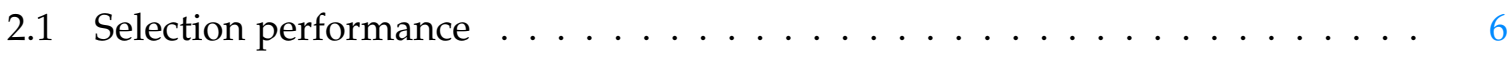

2.1.1 The Hick-Hyman Law . . . . . . . . . . . . . . . . . . 6

2.1 .2 Fits' Law . . . . . . . . . . . . . . . . . 6

2.1.2.1 Two-part formulations of Fitts' Law and gain . . . . . . . . 7

2.1.2.2 Fitts' Law formulations for 2-D static-target pointing tasks . . 9

2.1.2.3 Fitts' Law formulations for 3-D static-target pointing tasks . . 10

2.1.2.4 Fitts' Law formulations for 1-D moving-target pointing tasks . 11

2.1.2.5 Fitts' Law formulations for 2-D moving-target pointing tasks . 13

2.2 Subjective difficulty . . . . . . . . . . . . . . . . . . . 14

2.2.1 The importance of the rating method . . . . . . . . . . . . . . 14

2.2.2 Subjective difficulty in moving-target selection . . . . . . . . . . . . 14

2.3 Prediction of intention . . . . . . . . . . . . . . . . 15

2.3 .1 Scoring functions . . . . . . . . . . . . . . 15

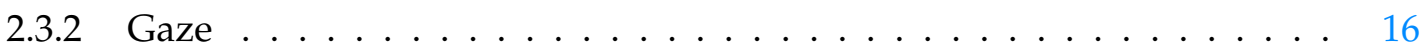

Chapter 3. Intention in UndiRected 3-D MOVING-TARget SELECTION $\ldots \ldots \ldots$

3.1 Hypotheses . . . . . . . . . . . . . . . . . . . . 19

3.1.1 Task-specific features for intention prediction in undirected movingtarget selection . . . . . . . . . . . . . . . . 19 
3.1.2 Generalizable features for intention prediction in undirected movingtarget selection . . . . . . . . . . . . . . . . . . 19

3.2 Pilot study . . . . . . . . . . . . . . . . . . . . . . . 19

3.3 Methods ................................ 19

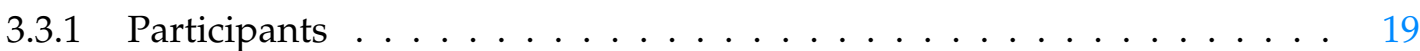

3.3 .2 Apparatus . . . . . . . . . . . . . . . . . . 20

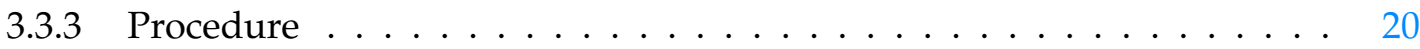

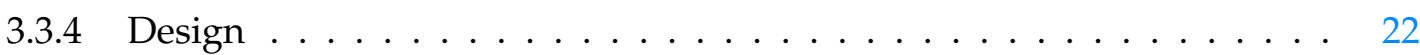

3.3 .5 Data integrity . . . . . . . . . . . . . . . 23

3.3.6 Predictive methods . . . . . . . . . . . . . . . . 23

3.3.6.1 Model comparison . . . . . . . . . . . . . . . 24

3.4 Task-specific feature analysis . . . . . . . . . . . . . . . . . 24

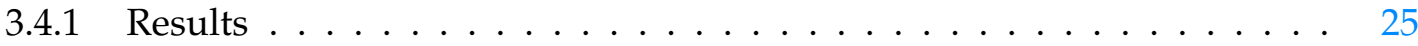

3.5 Generalizable feature analysis . . . . . . . . . . . . . . . . 26

3.5.1 Relative user-target features . . . . . . . . . . . . . . . . . . 27

3.5.1.1 Distance score feature . . . . . . . . . . . . . . . . 27

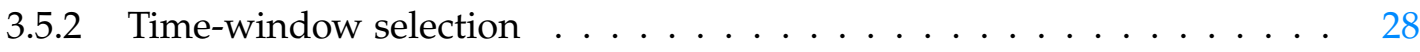

3.5 .3 Evaluation . . . . . . . . . . . . . . . . . . 30

3.5.4 Results and discussion . . . . . . . . . . . . . . . . 30

3.5.4.1 Generalizable user-target features . . . . . . . . . . . 30

3.5.5 Combined task-specific and generalizable features . . . . . . . . . . 31

3.6 Discussion . . . . . . . . . . . . . . . . . . . . 33

3.6.1 Task-specific features for intention prediction in undirected movingtarget selection . . . . . . . . . . . . . . . . 33

3.6.2 Generalizable features for intention prediction in undirected moving-

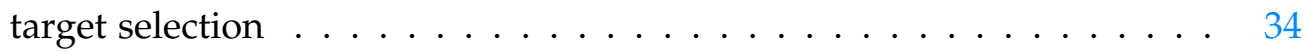

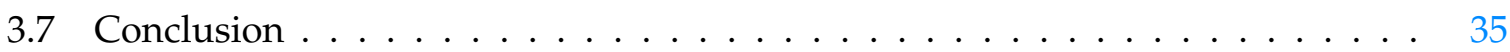

Chapter 4. Prospective difficulty of 2-D Static-target and moving-Target Selec-

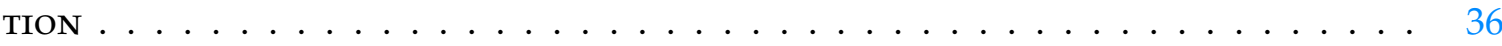

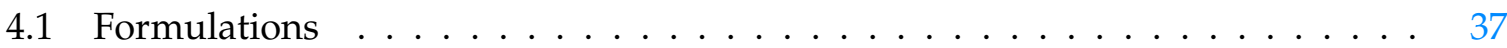

4.1.1 $P D$ formulation for 2-D static-target pointing tasks $\ldots \ldots \ldots \ldots$

4.1.2 $P D$ formulation for $1-D$ moving-target pointing tasks . . . . . . . . 37

4.1.3 PD formulation for 2-D moving-target pointing tasks . . . . . . . . . 37

4.2 Modeling . . . . . . . . . . . . . . . . . . . . 38

4.2 .1 Choice of $I D$ formulation $\ldots \ldots \ldots \ldots$

4.2.2 Formulations of prospective difficulty in 2-D static-target pointing tasks 39

4.2.3 Formulations of prospective difficulty in 1-D moving-target pointing tasks 41

4.2.4 Formulations of prospective difficulty in 2-D moving-target pointing tasks 42 
4.3 Methods . . . . . . . . . . . . . . . . . . . . . 43

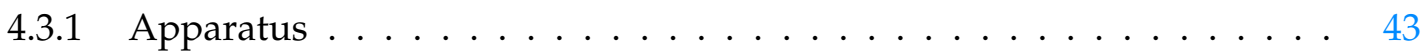

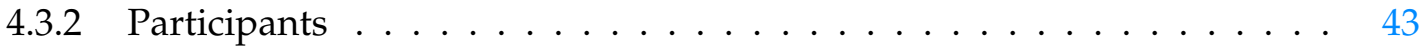

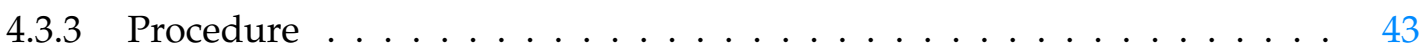

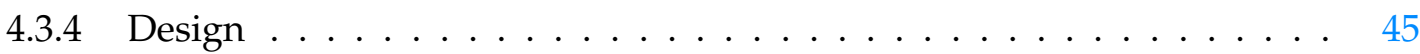

4.3 .5 Data integrity . . . . . . . . . . . . . . . . 46

4.3 .6 Statistical methods . . . . . . . . . . . . . . . 46

4.3.6.1 Models for inferential statistics . . . . . . . . . . . . . 46

4.3.6.2 Models for Regression Analysis . . . . . . . . . . . . . . 47

4.3 .7 Hypotheses . . . . . . . . . . . . . . . . . . . . 48

4.3.7.1 Static-target selection . . . . . . . . . . . . . . 48

4.3.7.2 Moving-target selection . . . . . . . . . . . . . . 48

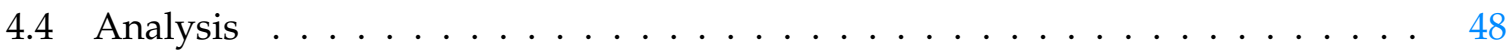

4.4 .1 Static-target block . . . . . . . . . . . . . . . 48

4.4.1.1 Inferential statistics . . . . . . . . . . . . . . 48

4.4.1.2 Regression analysis . . . . . . . . . . . . . . 50

4.4.1.3 Participant performance and self assessment . . . . . . . 52

4.4 .2 Moving-target block . . . . . . . . . . . . . . . 52

4.4.2.1 Inferential statistics . . . . . . . . . . . . . 52

4.4.2.2 Regression analysis . . . . . . . . . . . . . 55

4.4.2.3 Participant performance and self assessment . . . . . . . . 62

4.5 Discussion . . . . . . . . . . . . . . . . . . . . . 62

4.5.1 Prospective difficulty of static-target selection . . . . . . . . . . 63

4.5.2 Prospective difficulty of moving-target selection . . . . . . . . . . 64

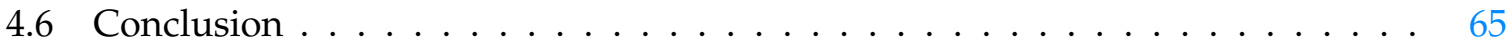

Chapter 5. Performance and intention in directed 3-D Moving-target Selection 67

5.1 Extending the 2-D formulae to 3 -D $\ldots \ldots \ldots \ldots \ldots \ldots$

5.2 Subjective difficulty and performance . . . . . . . . . . . . . 69

5.3 Predicting user intention in directed tasks . . . . . . . . . . . . . . . 70

5.4 Hypotheses . . . . . . . . . . . . . . . . . . 70

5.4.1 Moving-target selection performance in 3-D . . . . . . . . . . 70

5.4.2 Predicting user intention in 3-D moving-target directed-selection tasks . 71

5.5 Methods . . . . . . . . . . . . . . . . . . . . . 71

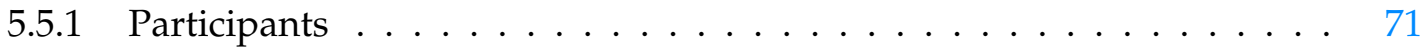

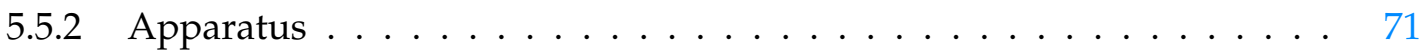

5.5 .3 Procedure ............................ 71

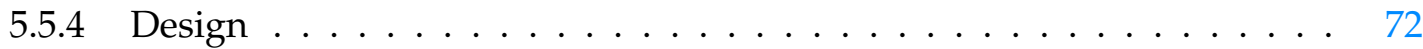

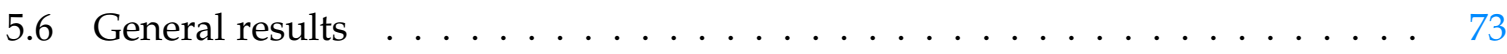


5.6 .1 Experimental issues . . . . . . . . . . . . . . . 73

5.6.1.1 Technical issues . . . . . . . . . . . . . . . . 74

5.6.1.2 Participant-wellness issues . . . . . . . . . . . . . . 74

5.6.1.3 Simulator sickness questionnaire . . . . . . . . . 75

5.7 Performance analysis . . . . . . . . . . . . . . . 76

5.7 .1 Exploratory data analysis $\ldots \ldots \ldots \ldots \ldots \ldots$

5.7.1.1 Between-block trial performance . . . . . . . . . . 76

5.7.1.2 Effects on MT for the successful trials in the 1-sphere block . . 77

5.7 .2 Regression analysis . . . . . . . . . . . . . . . 81

5.7.2.1 Change of coefficients per $V \ldots \ldots \ldots$. . . . . . 82

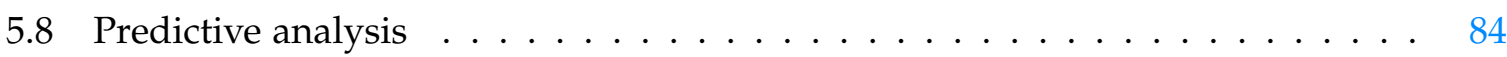

5.8 .1 Three-sphere trials . . . . . . . . . . . . . . . . . 85

5.8.1.1 Wand positions at the highlight frame . . . . . . . . 85

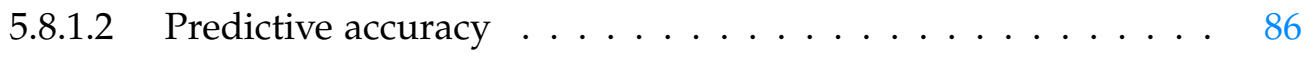

5.8 .2 Six-sphere trials . . . . . . . . . . . . . . . 86

5.8.2.1 Wand positions at the highlight frame . . . . . . . . . 86

5.8.2.2 Predictive accuracy ................ 86

5.9 Discussion . . . . . . . . . . . . . . . . . . . . . . 90

5.9.1 Moving-target selection performance in 3-D . . . . . . . . . . . 90

5.9.2 Predicting user intention in 3-D moving-target directed-selection tasks . 90

5.10 Conclusion . . . . . . . . . . . . . . . . . . . . . . . . . . . . . 91

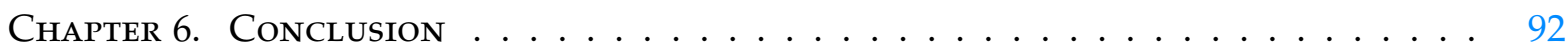

6.1 Limitations and future work . . . . . . . . . . . . . . . 93

6.1 .1 Intention prediction models . . . . . . . . . . . . . . . . . 93

$6.1 .2 P D$ prediction models . . . . . . . . . . . . . . . . 93

6.1.2.1 Two-dimensional static-target tasks . . . . . . . . . . . . . 94

6.1.2.2 Moving-target tasks . . . . . . . . . . . . . . . . . 94

6.1.2.3 Methods for assessing $P D \ldots \ldots$. . . . . . . . 95

6.1.3 Performance prediction models . . . . . . . . . . . . 95

6.1.3.1 Target velocity . . . . . . . . . . . . . . 95

6.1 .3 .2 Target distance . . . . . . . . . . . . . . . . 95

6.1.3.3 Additional measures of performance . . . . . . . . . . . 95

6.1 .4 General issues . . . . . . . . . . . . . . . . . . . . . 96

6.1.4.1 Target shape . . . . . . . . . . . . . . 96

6.1 .4 Target acceleration ..................... 96

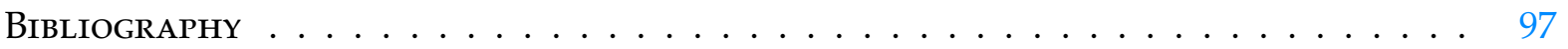


ANNEXE A. RÉSUMÉ ÉTENDU EN FRANÇAIS $\ldots \ldots \ldots \ldots \ldots \ldots$ A1

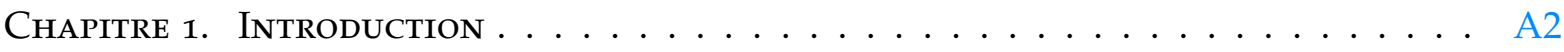

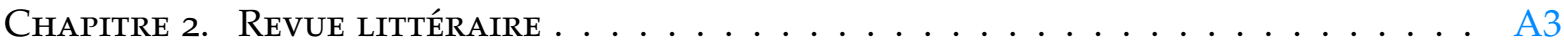

2.1 Performance des tâches de sélection $\ldots \ldots \ldots \ldots \ldots \ldots \ldots \ldots$ A3

2.1.1 La loi de Hick-Hyman . . . . . . . . . . . . . . . A3

2.1 .2 La loi de Fitts . . . . . . . . . . . . . . . . . . . A3

2.1.2.1 Formules en deux parties de la loi de Fitts et le gain contrôle-

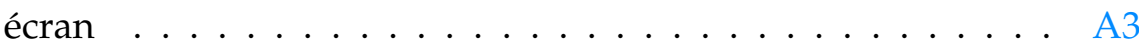

2.1.2.2 Formules 2-D de la loi de Fitts pour des tâches de pointage

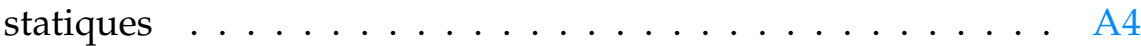

2.1.2.3 Formules 3-D de la loi de Fitts pour des tâches de pointage

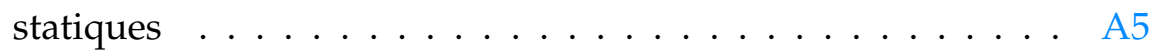

2.1.2.4 Formules de la loi de Fitts pour des tâches de pointage sur des cibles en mouvement 1-D . . . . . . . . . . . A A5

2.1.2.5 Formules de la loi de Fitts pour des tâches de pointage sur des cibles en mouvement 2-D . . . . . . . . . . . . . . . A5

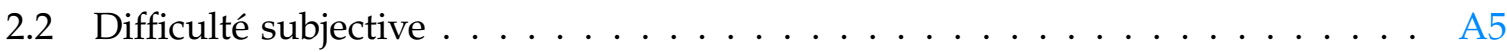

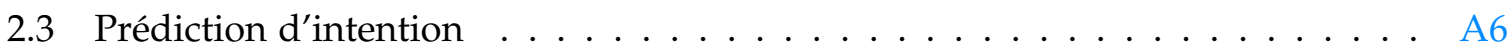

2.3 .1 Les fonctions de scoring $\ldots \ldots \ldots \ldots \ldots \ldots \ldots \ldots \ldots$ A6

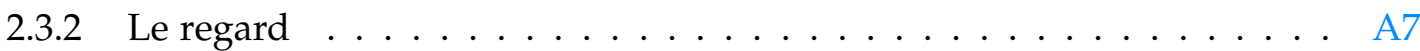

ChAPITRE 3. L'INTENTION DANS LA SÉLeCTION NON-DiRigéE DE CIBLES EN MOUVEMENT

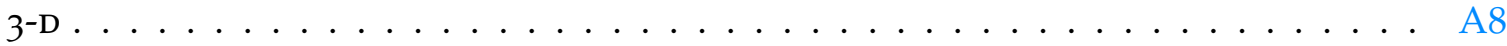

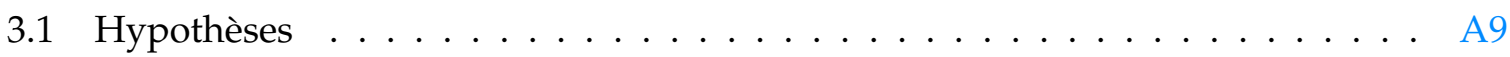

3.1.1 Caractéristiques spécifiques à la tâche pour la prédiction d'intention dans des tâches de sélection non-dirigée de cibles en mouvement $\ldots$ A9

3.1.2 Caractéristiques généralisables pour la prédiction d'intention dans des tâches de sélection non-dirigée de cibles en mouvement . . . . . . . A9

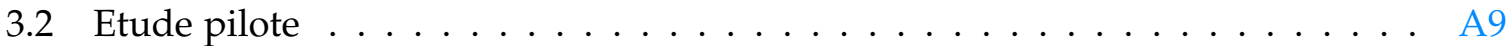

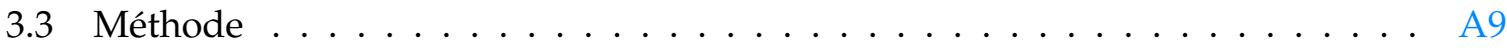

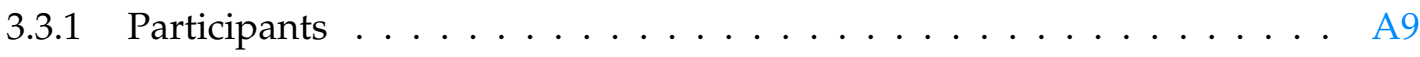

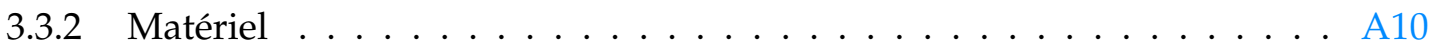

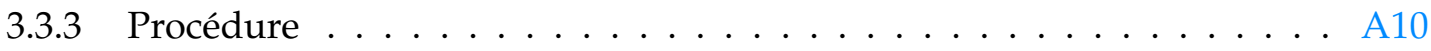

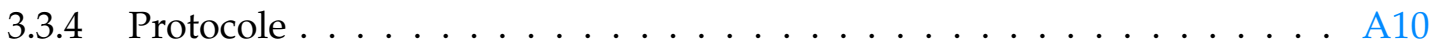

3.3 .5 Discussion . . . . . . . . . . . . . . . . . . . . A11

3.3.6 Caractéristiques spécifiques à la tâche pour la prédiction d'intention dans des tâches de sélection non-dirigée de cibles en mouvement . . . A11 
3.3.7 Caractéristiques généralisables pour la prédiction d'intention dans des tâches de sélection non-dirigée de cibles en mouvement . . . . . . . . . A11

Chapitre 4. Difficulté prospective de tÂChes 2-D de Cibles Statiques ET EN

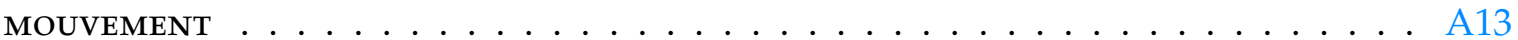

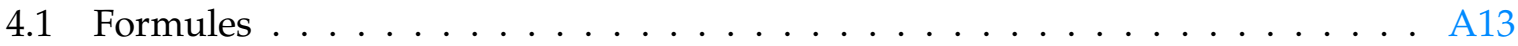

4.1.1 Formule de DP pour des tâches 2-D de sélection de cibles statiques . . A13

4.1.2 Formule de DP pour des tâches 1-D de sélection de cibles en mouvement A14

4.1.3 Formule de DP pour des tâches 2-D de sélection de cibles en mouvement A14

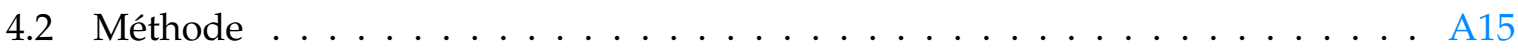

4.2 .1 Matériel . . . . . . . . . . . . . . . . . A15

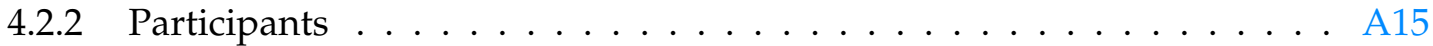

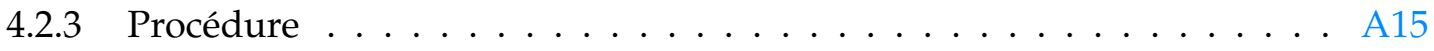

4.2 .4 Protocole . . . . . . . . . . . . . . . . . A16

4.3 Discussion . . . . . . . . . . . . . . . . . A16

4.3.1 Prospective difficulty of static-target selection . . . . . . . . . . A17

4.3.2 Difficulté prospective de la sélection de cibles en mouvement . . . . . . A17

Chapitre 5. Performance et intention dans la sélection dirigée de Cibles en MOUVEMENT EN 3 -D . . . . . . . . . . . . . . . . . . A19

5.1 L'extension des modèles 2-D en 3 -D . . . . . . . . . . . . . . . . . . . A19

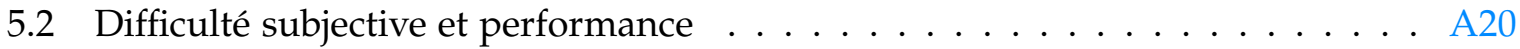

5.3 Prédiction de l'intention de l'utilisateur dans de tâches dirigées . . . . . . . . . A21

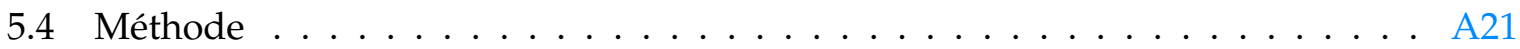

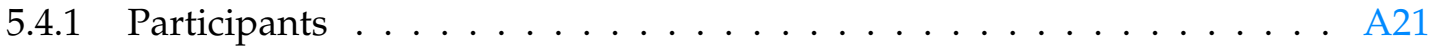

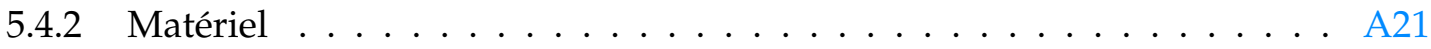

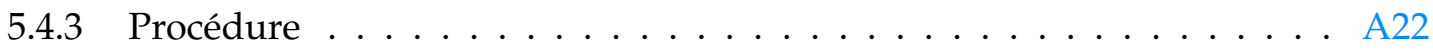

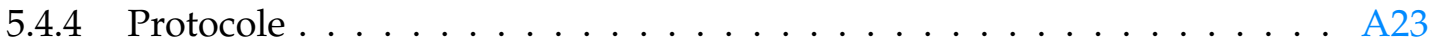

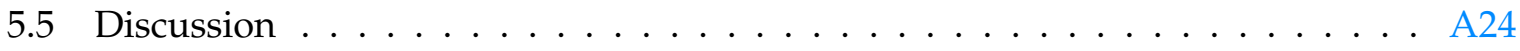

5.5.1 Performance de la sélection 3-D de cibles en mouvement . . . . . . . . A24

5.5.2 Prédiction d'intention dans des tâches 3-D de sélection dirigée de cibles en mouvement . . . . . . . . . . . . . . . . . A24

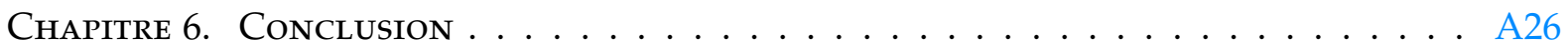




\section{LIST OF TABLES}

Table 3.1 Accuracy and 95\% confidence intervals for the evaluated feature-sets . 25

Table 3.2 Tree size, number of leaves, accuracy and 95\% confidence intervals for the evaluated generalizable moving-target feature-sets . . . . . . . . 30

Table 3.3 Accuracy difference and 95\% confidence intervals for the evaluated generalizable moving-target feature-sets. Asterisks $\left({ }^{*}\right)$ denote a significant difference $(\alpha=0.05) \ldots \ldots \ldots$. . . . . . . . . . . 31

Table 3.4 Tree size, number of leaves, accuracy and $95 \%$ confidence intervals for the evaluated target-based feature-sets. . . . . . . . . . . . 33

Table 3.5 Accuracy difference and 95\% confidence intervals for the target-based feature-sets. Asterisks $\left.{ }^{*}\right)$ denote a significant difference $(\alpha=0.05)$, dots $($.$) denote a marginal difference (\alpha=0.1) \ldots \ldots 33$

Table $4.1 \quad$ Regression estimates for $\overline{P D}=a+b I D \ldots \ldots \ldots \ldots$

Table $4.2 \quad$ Regression estimates for $\overline{P D}=a_{\theta}+b I D \ldots \ldots \ldots \ldots 2$

Table $4.3 \quad$ Regression estimates for $\overline{P D}=a_{V}+b_{V} \sqrt{D}+c_{V} I D \ldots \ldots \ldots 7$

Table 4.4 Least-squares estimates for the regressions of $\hat{a}_{V}, \hat{b}_{V}$, and $\hat{c}_{V}$, from Table 4.3 , on $V \ldots \ldots \ldots \ldots \ldots \ldots \ldots \ldots$

Table 4.5 Hierarchical regression $\overline{P D}_{i} \sim \mathcal{N}\left(a_{V[i]}+b_{V[i]} \sqrt{D_{i}}+c_{V[i]} I D_{i}, \sigma^{2}\right) \ldots \quad 58$

Table 4.6 Regression estimates for $\overline{P D}=a_{V}+b_{V} \sqrt{D_{s}}+c_{V} \sqrt{D_{m}}+d_{V} I D_{m} \ldots \ldots 9$

Table $4.7 \quad$ Regression estimates for $\overline{P D}=a_{V}+b \sqrt{D_{s}}+c_{V} \sqrt{D_{m}}+d I D_{m} \ldots \ldots 0$

Table 4.8 Least-squares estimates for the regressions of $\hat{a}_{V}$ and $\hat{c}_{V}$, from Table 4.7,

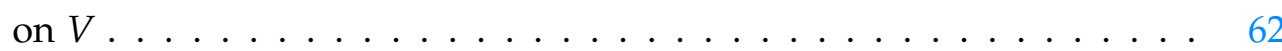

Table 4.9 Hierarchical regression $\overline{P D}_{i} \sim \mathcal{N}\left(a_{V[i]}+b \sqrt{D_{s i}}+c_{V[i]} \sqrt{D_{m i}}+d I D_{m i}, \sigma^{2}\right) 63$

Table 5.1 Regression estimates for $\overline{P D}=a_{V}+b_{V} \sqrt{D_{s}}+c_{V} \sqrt{D_{m}}+d_{V} I D_{m} \ldots . \quad$. 83

Table 5.2 Least-squares estimates for the regressions of the point estimates from Table 5.1- $\hat{a}_{V}=\operatorname{Mdn}\left(a_{V}\right), \hat{b}_{V}=\operatorname{Mdn}\left(b_{V}\right), \hat{c}_{V}=\operatorname{Mdn}\left(c_{V}\right)$, and $\hat{d}_{V}=$ $M d n\left(d_{V}\right)$-on target speed $V . \ldots \ldots \ldots$

Table 5.3 Hierarchical regression

$\overline{M T}_{i} \sim \mathcal{N}\left(a_{V[i]}+b_{V[i]} \sqrt{D_{s i}}+c_{V[i]} \sqrt{D_{m i}}+d_{V[i]} I D_{m i}, \sigma^{2}\right) \ldots \ldots 84$ 


\section{LIST OF FIGURES}

Figure 2.1 Two-dimensional task with a bivariate target. The red disc indicates the cursor; the green rectangle represents a target with width $W$, and height $H$. $D$ is the cursor-target distance, and $\theta$ is the target angle. .

Figure 2.2 Three-dimensional trivariate target. The red disc indicates the cursor; the green rectangular cuboid represents a target with width $W$, height $H$, and depth $\not D$. $D$ is the cursor-target distance, $\alpha$ is the $z-x$ azimuth angle, and $\theta$ is the altitude angle. . . . . . . . . . . 11

Figure $3.1 \quad$ Experimental setup of the pilot study. . . . . . . . . . . . . . 20

Figure 3.2 Experimental setup with an array two spheres . . . . . . . . . . . 21

Figure 3.3 Possible row positions-left, center and right-with respect to the user in the two-sphere block . . . . . . . . . . . . . . . 22

Figure 3.4 Decision tree for feature-set 4, suggesting that participants based their decisions only on sphere size, with a preference for the right sphere. Leaves represent prediction outcomes $\left(\mathrm{sph}_{1}\right.$ is the left sphere, and $\mathrm{sph}_{2}$ is the right sphere), while the other nodes represent tested attributes $\left(r_{1}\right.$ or $\left.r_{2}\right)$. The numbers in parenthesis within the leaves represent the total number of instances that fall into that leaf, over the number of incorrectly predicted instances among these instances. . . . 26

Figure $3.5 \Delta$ dot vs. time. Each line corresponds to a trial, colored according to the selected sphere. The plot has been trimmed to the $5^{\text {th }}$ percentile of the selection times $(2.35 \mathrm{~s}) \ldots \ldots \ldots \ldots \ldots$

Figure 3.6 Generated decision tree for feature-set $\{\overline{\Delta d o t}, \overline{\Delta D}\}$. The numbers in parenthesis within the leaves represent the total number of instances that fall into that leaf, over the number of incorrectly predicted instances among these instances. . . . . . . . . . . . . 32

Figure 4.1 Measurements relevant to the target-selection tasks considered in this

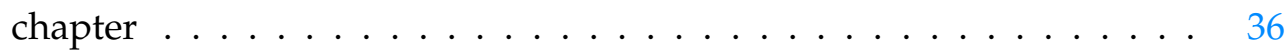

Figure 4.2 Two-dimensional moving-target model . . . . . . . . . . . . . . 42

Figure 4.3 Screenshot of a moving-target question with $\phi=45^{\circ} \ldots \ldots$ 
Figure 4.4 Static-target task . . . . . . . . . . . . . . . . 45

Figure 4.5 Moving-target task . . . . . . . . . . . . . . . . . 45

Figure 4.6 Main-effect coefficient plot for the fitted ordered probit model on statictarget $P D$ ratings . . . . . . . . . . . . . . . . 49

Figure 4.7 Two-way interaction coefficient plot for the fitted ordered probit model

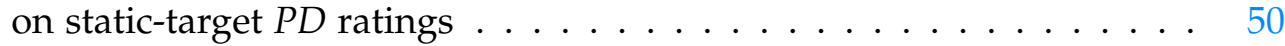

Figure $4.8 \quad$ Regression for $\overline{P D}=a+b I D \ldots \ldots \ldots \ldots \ldots \ldots$

Figure 4.9 Regression for $\overline{P D}=a_{\theta}+b I D \ldots \ldots \ldots \ldots$. . . . . . . 52

Figure 4.10 Main-effect coefficient plot for the fitted ordered probit model on moving-target $P D$ ratings $\ldots \ldots \ldots 53$

Figure 4.11 Two-way interaction coefficient plot for the fitted ordered probit model on moving-target $P D$ ratings $\ldots \ldots \ldots$. . . . . . . . . . . 54

Figure 4.12 Regression for $\overline{P D}=a_{V}+b_{V} \sqrt{D}+c_{V} I D \ldots \ldots \ldots \ldots$

Figure 4.13 Regression for $\overline{P D}=a_{V}+b \sqrt{D_{s}}+c_{V} \sqrt{D_{m}}+d I D_{m} \ldots \ldots \ldots 1$

Figure 5.1 Three-dimensional moving-target model . . . . . . . . . . . . 68

Figure 5.2 Identical model parameters $V, D_{m}$, and $D_{s}$ for three different $\gamma$ rotations of $\mathbf{V}$ around $\mathbf{D} \ldots \ldots \ldots \ldots$. . . . . . . . . . . . 68

Figure 5.3 Experimental setup with six spheres. Left, the sphere starting positions, middle the spheres approximately at their controlled positions, right the spheres after the goal sphere gets highlighted. The green sphere represents $s p h_{\underline{0}} . \ldots \ldots \ldots \ldots \ldots \ldots \ldots \ldots \ldots \ldots \ldots \ldots \ldots \ldots \ldots$

Figure 5.4 Distributions of the pre- and post-experiment assessment scores of the sse by scale. Lower and upper lines of the boxes represent the first and third quantiles, their distance called the inter-quantile range (IQR), thick box lines represent the median values, upper and lower whiskers represent values that are within 1.5IQR of the box hinges. Points represent values that are greater than the third quantile plus

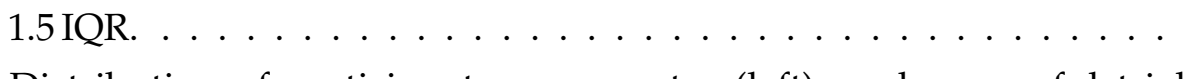

Figure 5.5 Distribution of participant success rates (left), and successful trial completion times (right) per experimental block . . . . . . . . . 76

Figure 5.6 Distributions of movement times for the successful trials per experimental factor in the 1 -sphere block . . . . . . . . . . . . 78

Figure 5.7 Movement times per $\gamma$ angle for the successful trials in the 1-sphere block. . . . . . . . . . . . . . . . 79

Figure 5.8 Front and right views of the distribution of wand positions at the frame where the sphere was highlighted in the 1 -sphere trials . . . . . . . . 
Figure 5.9 Movement times for each of the distances between the target sphere and the wand at the highlight frame $\left\|P_{w}-P\right\|$, for the successful trials in the 1-sphere block. The top bars show the distribution of $\left\|P_{w}-P\right\|$ binned every $0.025 \mathrm{~m}$. The orange lines represent the distance between the target sphere and $P_{w, 0} \pm 0.05 \mathrm{~m} \ldots \ldots \ldots$. . . . . . . . 81

Figure 5.10 Residuals $(\overline{M T}-\widehat{M T})$ vs. fitted values $(\widehat{M T})$ for the $\overline{M T} \sim \mathcal{N}\left(a_{V}+\right.$ $\left.b_{V} \sqrt{D_{s}}+c_{V} \sqrt{D_{m}}+d_{V} I D_{m}, \sigma^{2}\right)$ regression. The dotted lines repre-

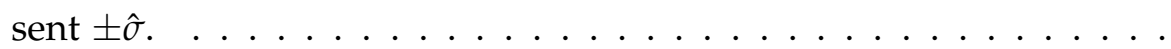

Figure 5.11 Front and right views of the distribution of wand positions at the frame where the goal sphere was highlighted in the 3 -sphere trials . . . . . .

Figure 5.12 Three-sphere accuracy comparison for the $\widehat{T} S$ core, and $d S$ core predictors for trial percentages $0.1 T, 0.2 T, \cdots, 0.9 T, T$, using different $N$, and decay parameters. The dotted lines represent the accuracy given by chance, i.e. $1 / 3 \ldots \ldots \ldots \ldots \ldots \ldots$

Figure 5.13 Front and right views of the distribution of wand positions at the frame where the goal sphere was highlighted in the 6-sphere trials . . . . . .

Figure 5.14 Three-sphere accuracy comparison for the $\widehat{T} S$ core, and $d S c o r e$ predictors for trial percentages $0.1 T, 0.2 T, \cdots, 0.9 T, T$, using different $N$, and decay parameters. The dotted lines represent the accuracy given by chance, i.e. $1 / 3 \ldots \ldots \ldots \ldots \ldots \ldots$ 


\section{ACKNOWLEDGMENTS}

I wish to express my gratitude to my advisers, James Oliver, Frédéric Mérienne, and Samir Garbaya, for their trust and guidance throughout this joint PhD program. I am especially thankful to Dr. Oliver who met with me well-nigh every week during these years, and was always supportive and enthusiastic about my research, writing, and personal accomplishments.

I am also grateful to my international committee for having shared their expertise in their domains throughout my doctoral studies. In particular, I want to thank Michael Dorneich who mentored me in academic life in general, and Jonathan Kelly who also actively collaborated with me and mentored me in research. I also want to thank Vincent Hugel and Samir Otmane for reviewing this dissertation.

A venture like this one would not have been possible without all my family, friends, colleagues, and personnel in France, the USA, and Colombia. Thanks to you, these five years have been an incredibly enriching experience. I am afraid that no proper acknowledgment can be given in such a short space.

I must also acknowledge the profound role that my parents have played in this achievement, by preparing me, perhaps unknowingly, for this accomplishment. I also want to thank my sister, Laura, future real doctor, for her love, and her active and tacit encouragement.

Last, but never least, I am obliged to my wife, Catherine, who blissfully followed me in this endeavor, and gave me the most significant result of these years-Gaël and Inés. 


\section{AbStRACt}

Selection of moving targets is a common task in human-computer interaction (HCI), and more specifically in virtual reality (VR). In spite of the increased number of applications involving moving-target selection, HCI and VR studies have largely focused on static-target selection. Compared to its static-target counterpart, however, moving-target selection poses special challenges, including the need to continuously and simultaneously track the target and plan to reach for it, which may be difficult depending on the user's reactiveness and the target's movement. Action prediction has proven to be the most comprehensive enhancement to address moving-target selection challenges. Current predictive techniques, however, heavily rely on continuous tracking of user actions, without considering the possibility that targetreaching actions may have a dominant pre-programmed component-this theory is known as the pre-programmed control theory.

Thus, based on the pre-programmed control theory, this research explores the possibility of predicting moving-target selection prior to action execution. Specifically, three levels of action prediction are investigated: action performance, prospective action difficulty, and intention. The proposed performance models predict the movement time (MT) required to reach for a moving target in 2-D and 3-D space, and are useful to compare users and interfaces objectively. The prospective difficulty $(P D)$ models predict the subjective effort required to reach for a moving target, without actually executing the action, and can therefore be measured when performance can not. Finally, the intention models predict the target that the user plans to select, and can therefore be used to facilitate the selection of the intended target.

Intention prediction models are developed using decision trees and scoring functions, and evaluated in two vR studies: the first investigates undirected selection (i.e., tasks in which the users are free to select an object among multiple others), and the second directed selection (i.e., the more common experimental task in which users are instructed to select a specific object). PD models for 1-D, and 2-D moving-target selection tasks are developed based on Fitts' Law, and evaluated in an online experiment. Finally, MT models with the same structural form of the aforementioned $P D$ models are evaluated in a 3-D moving-target selection experiment deployed in VR. Aside from intention predictions on directed selection, all of the explored models yield relatively high accuracies-up to $\sim 78 \%$ predicting intended targets in undirected tasks, $\hat{R}^{2}=.97$ predicting $P D$, and $\hat{R}^{2}=.93$ predicting $M T$. 


\section{CHAPTER 1}

\section{INTRODUCTION}

\subsection{Moving-target selection}

Selection of moving targets is a common task in human-computer interaction (HCI) and more specifically in virtual reality (VR). Targets may move independently from user input, as in interactive, or "clickable," video [Ilich 2009; Hasan et al. 2011; Silva et al. 2012], and air traffic control displays [Mould and Gutwin 2004; Hasan et al. 2011]. Targets may also move relative to the user, a case in point being navigation in VR [Mould and Gutwin 2004] and augmented reality (AR) [You et al. 2012]. In some applications, including video games [Mould and Gutwin 2004; Pavlovych and Gutwin 2012] and interactive 3-D simulations [Mould and Gutwin 2004; Hasan et al. 2011], both kinds of movements are present.

In spite of the increased number of applications involving moving-target selection, HCI and VR studies have largely focused on static-target selection. In the taxonomy for VR manipulation tasks presented by Poupyrev et al. [1997], selection parameters such as target size and distance are given some level of detail, including measurement variables, whereas target movement is classified under "other parameters," without any further description. In the more recent taxonomies of 3-D interaction techniques by Bowman et al. [2004], and 3-D selection techniques by Argelaguet and Andujar [2013], target motion is not included as a parameter for target selection. This example is, perhaps, reflective of the numerous HCI studies on static-target selection based on Fitts' Law [Fitts 1954], whose inputs are target size and distance only.

\subsubsection{Moving-target selection challenges}

Moving-target selection poses special challenges compared to its static-target counterpart. Unlike static-target selection, which can be executed without ongoing visual control under certain conditions [Hoffmann and Chan 2012], the nature of moving-target selection requires the user to continuously and simultaneously track targets and plan to reach for them [Hasan et al. 2011], which may be difficult considering the inherent speed and precision limitations of the human sensory-motor system [Shadmehr et al. 2010]. In some applications targets may move along unpredictable paths [Ilich 2009; Hasan et al. 2011; Pavlovych and Gutwin 2012; 
You et al. 2012; Ortega 2013], with changing speeds [Pavlovych and Gutwin 2012; You et al. 2012], making the task more challenging. Additionally, certain combinations of target and viewport motions may cause targets to fall out of the user's field of view, become occluded, or change in visible size, this problem is illustrated in interactive sport-videos in which players can be selected while moving [Ilich 2009]. Even in cases in which target visibility, size, and velocity are constant, there is a critical speed beyond which selecting the target becomes impossible [Hoffmann 1991].

Finally, common HCI challenges are exacerbated in VR moving-target selection. End-to-end latency (the delay between user input and system output), for example, is often revealed to users when tracking a moving target [Reddy 1994]. More importantly, the presence of latency sharply affects moving-target reaching accuracy and, to a lesser degree, moving-target tracking [Pavlovych and Gutwin 2012]. Similarly, vR induced symptoms such as sensory conflict, simulator sickness, confusion, and frustration [Cobb et al. 1999] are likely worsened in moving-target selection.

\subsubsection{Moving-target selection enhancements}

Ilich [2009] identifies two major categories for moving-target selection enhancement: pointer enhancement, and target enhancement. Ilich also suggests the general "task simplification" category, but the strategies classified in this category are not described since they can also be considered as pointer and target enhancements.

According to Ilich, pointer enhancement is possible through an increase in the speed or area of the pointer, as well as an added affinity to certain targets. The sole increase in pointer size extends the time window for selection when targets are to be intercepted [Tresilian 2005], and may reduce the effective target distance on target-chase tasks [Ilich 2009], but it may also affect target visibility and acquisition accuracy, especially when targets are small-this issue is known in touch screens as the "fat finger" problem [Holz and Baudisch 2010]. The increase in pointer speed in the form of velocity control has been shown to increase the selection accuracy in tasks with a single moving-target [Jagacinski et al. 1980], but such control techniques, also available in static-target selection, are known to suffer from a decrease in positioning precision as the reaching distance increases [Bowman et al. 2004, p. 162]. The last type of pointer enhancement, pointer-target affinity techniques, has been shown to be successful in increasing selection accuracy in tasks with multiple targets by linking the pointer to targets whose angular distances [de Haan et al. 2005] and euclidean distances [Ortega 2013] to the pointer decrease more rapidly, these techniques are described in depth in Section 2.3.1.

Concerning target enhancement, Ilich describes three possible techniques: target expansion, target repositioning, and target speed decrease. Target expansion strategies, such as the Comet [Gunn et al. 2009; Hasan et al. 2011], and AttachedShock [You et al. 2012, 2014], enhance pointing by extending the selectable target area to include a movement trail left behind 
each target, but suffer from clutter and overlap when the number of selectable targets is increased. Target repositioning techniques, such as target Ghost [Hasan et al. 2011], which creates static proxies for each target upon activation, exhibit the same trade-off between enhanced performance but increased clutter and overlap. The last target enhancement, speed decrease, has been successfully implemented in techniques that completely stop targets [Ilich 2009; Al Hajri et al. 2011], thus reducing the task to static selection. In spite of their benefits, these target enhancements may be undesirable in certain applications, especially those that strive for realism.

Apart from pointer-target affinity techniques, the enhancements proposed by Ilich provide only partial solutions to the challenges in moving-target selection, while aggravating other existing challenges, or introducing new ones. The reason pointer-target affinity stands out is that it provides a way to anticipate the intended target and enhance the pointer only with respect to that target. Such a principle is not restricted to pointer enhancement, prediction of targets and motion endpoints has been suggested to address clutter and overlap in target enhancement techniques for both static [McGuffin and Balakrishnan 2005; Lank et al. 2007; Wonner et al. 2011] and moving-target [Hasan et al. 2011] selection tasks.

\subsubsection{Control strategies in moving-target selection}

The pointer-target affinity techniques described above are based on the principle that the user is constantly following their intended target. According to Tresilian [2005], however, the motion required to reach a moving-target has a pre-programmed control component that may be dominant over on-line control, especially when the motion must be executed rapidly. In other words, such a motion is minimally influenced by external sensory information once the motor commands are issued.

Thus, based on the pre-programmed control theory, this research explores the possibility of predicting moving-target selection prior to action execution. More specifically, three levels of action prediction are investigated: action performance, prospective action difficulty, and intention. These levels of prediction are subsequently described with respect to existing action models, including the seven stages of action [Norman 1986, 2002], and the aforementioned pre-programmed control theory.

Among these predictions, performance is assessed during action execution, the lowest cognitive stage of action. It refers to quantitative measures of task execution, which can be used to evaluate users, interfaces, and interaction techniques objectively. In particular, in accordance with the main HCI body of static-target selection studies, the focus is on predicting the movement time $(M T)$ required to reach the target. For static-target selection, MT has been shown to be positively and linearly related to the index of difficulty, $I D=\log _{2}(2 D / W)$, where $D$ and $W$ are the target's size and distance, this relation is known as Fitts' law [Fitts 1954]. Unfortunately, as shown in Chapter 2, the existing formulae for MT in moving target 
selection are limited to 1-D (i.e., target velocity directly toward or away from the cursor) and lack a simple expression of difficulty similar to the aforementioned ID. One of the aims of this research is to extend the $M T-I D$ paradigm to 2-D, and 3-D moving-target selection.

Prospective difficulty $(P D)$, refers to subjective assessments of difficulty evaluated prior to action execution, which typically occurs during the higher level stage of action specification. Since prospective judgments do not require action execution, they may be measured even when performance can not, due to factors such as task feasibility, low occurrence, or even practicality. Unfortunately, direct assessment of $P D$ requires either task interruption, or the usage of sensors such as EEG [Kourtis et al. 2012], which may be invasive and are generally not available in all VR setups. Nonetheless, since $P D$ is assessed during action preparation, its value is probably related to the task parameters that also affect performance. In particular, it has been shown that $P D$, and ID are related for 1-D static-target selection tasks [Slifkin and Grilli 2006; Grilli 2011], but so far this relation has not been explored in moving-target selection. Given the usefulness of $P D$, this research also aims to extend the $P D-I D$ paradigm to moving-target selection.

Finally, intention, a general action that a user plans to execute to achieve a goal, precedes action specification and is therefore at the highest cognitive level among these three measurements. In this work, the scope of such intentions is limited to the target the user plans to select. ${ }^{1}$ Similar to $P D$, direct assessment of intention requires task interruptions, or inference via proxy measures that relate user actions to targets (user-target states), such as gaze. Based on the principle of pre-programmed action, however, it is hypothesized that users form their intentions by minimizing their prospective effort.

Minimizing prospective effort to form intentions can be observed in undirected selection tasks, i.e., tasks in which users are free to choose an object among multiple others, as opposed to the more (experimentally) common directed tasks, in which users are instructed to select a specific target. In static-target selection, given the correlation between $P D$ and $I D$, minimizing prospective effort is hypothetically equivalent to minimizing $I D$.

Therefore, in terms of intention, this research aims to a) test the hypothesis that users form their intentions by minimizing their prospective effort, and b) evaluate the predictive accuracy of different user-target states as proxies for intention.

\footnotetext{
${ }^{1}$ This scope contrasts with some of the previous work in which intention refers to the usage given to a target following selection [Mandryk and Lough 2011; Song et al. 2013; Ruiz and Lank 2014].
} 


\subsection{Research Layout}

The remainder of this dissertation is structured as follows,

1. Chapter 2 presents the relevant literature related to static-target and moving-target selection, prospective difficulty, and the usage of user-target states as prediction inputs.

2. Chapter 3 presents the results of the first user study. This study evaluates the hypothesis that users form their intentions by minimizing their prospective effort as described by $I D$, in undirected moving-target selection tasks in VR. Since ID is a task-specific feature, limited in usefulness to undirected tasks, it was complemented with generalizable user-target features that can also be used in directed tasks.

3. Chapter 4 develops $P D$ models for 2-D static-target and moving-target selection. These models are validated with the results of an online user study.

4. Chapter 5 extends the $P D$ models developed in Chapter 4 to 3 -D, and evaluates their usefulness as predictors of $M T$, and intention.

5. Chapter 6 presents the contributions, future work and conclusions of the dissertation. 


\section{CHAPTER 2}

\section{LITERATURE REVIEW}

\subsection{Selection performance}

Target-selection performance in HCI is usually studied using information-theoretic models, including Fitts' Law [Fitts 1954] and, to a lesser degree, the Hick-Hyman Law [Hick 1952; Hyman 1953]. Card, English, and Burr [1978] were the first to use Fitts' Law in HCI to compare the movement times (MT) of different input devices, and Card, Moran, and Newell [1983] presented both laws as part of the operating principles of the Model Human Processor.

\subsubsection{The Hick-Hyman Law}

The Hick-Hyman Law relates the reaction time $(R T)$ required to make a choice to the number $(n)$ and probability $(p)$ of each of the possible choices such that,

$$
R T=a+b \sum_{i}^{n} p_{i} \log _{2}\left(\frac{1}{p_{i}}+1\right)
$$

where $a$ and $b$ are empirically determined coefficients. If all choices have equal probability $(p=1 / n)$, Equation (2.1) is reduced to

$$
R T=a+b \log _{2}(n+1)
$$

Notice that the $R T$ describes only the time to make the choice, and not the $M T$ required to execute the selection.

\subsubsection{Fitts' Law}

Fitts' Law relates the mean movement time $(\overline{M T})$ required to reach a target to the target's index of difficulty (ID), such that

$$
M T=a+b I D,
$$


where $a$ and $b$ are empirically determined coefficients. ID gives an objective measure of the difficulty involved in pointing at a target as a function of the ratio between the target's distance $(D),{ }^{1}$ and its size $(W)$. The two most common ID formulations are Fitts' original formulation [1954]

$$
I D_{F}=\log _{2}\left(\frac{2 D}{W}\right),
$$

and the so-called Shannon formulation, suggested by MacKenzie [1989],

$$
I D_{S h}=\log _{2}\left(\frac{D}{W}+1\right)
$$

Fitts' Law has been extensively used in HCI, psychology, and related fields to model human performance (for compendia see MacKenzie [1992], and Guiard and Beaudouin-Lafon [2004]), but there is an ongoing debate concerning the correctness and usefulness of these two ID formulations [Drewes 2010; Hoffmann 2013; MacKenzie 2013]. Regardless of these issues, as noted by Drewes [2010], the use of both formulae should lead to similar results and, as noted in a personal communication with Hoffmann [2014], the differences may be of statistical but not practical significance. In any case, it is important to consider that both models represent an approximation of a more complex reality [Drewes 2010]. Citing a popular phrase of the late statistician George Box, "essentially all models are wrong, but some are useful" [Box and Draper 1987, p. 424].

\subsubsection{Two-part formulations of Fitts' Law and gain}

Welford et al. [1969] suggested that pointing could be separated in two control processes: a motor, or distance-covering process, and a visual, or homing-in process. To account for these two processes, Welford and colleagues proposed the following variation of Fitts' Law:

$$
M T=a+b \log _{2}(D)-c \log _{2}(W) .
$$

Inspired by Kopper et al. [2010], Shoemaker et al. [2012] reformulate Welford's model by introducing the ratio $k=c / b$, which encapsulates the relative impact of the two-parts of Welford's formula, yielding a model similar in form to Equation (2.3), such that

$$
M T=a+b \log _{2}\left(\frac{D}{W^{k}}\right) .
$$

In their analyses, Shoemaker et al. [2012] found that two-part formulations described MT better than one-part models when pointing at targets with different levels of control-display gain $(G$, the ratio of the cursor speed to the input speed). Additionally, based on different

\footnotetext{
${ }^{1}$ Traditionally researchers refer to this quantity as amplitude $(A)$, referring to the movement amplitude required to reach for the target, in this work, the term distance $(D)$ is preferred, particularly because in moving-target selection $D$, but not $A$, is known beforehand.
} 
regression estimates, they also observed that $k$ increased linearly with $G$. Shoemaker and colleagues also noted that $c$ "increases quite consistently with gain," while $b$ "stays relatively constant," but, contrary to the $k-G$ relation, no comment on the possible form of the $b-G$ and $c-G$ relations, or on the evident increase of $a$ with $G$, was given.

Even though the understanding of the $k-G$ relation may be important to characterize the relative impact of each part of Welford's model per $G$ level, and gauge how far is the interaction from being modeled by Fitts' original formulation (which is essentially Equation (2.7) with $k=1$ ), understanding the $a-G, b-G$, and $c-G$ relations allow comparing MT between $G$ levels, as well as generalizing the results to other experimental conditions. Regression analyses for $a-G, b-G$, and $c-G$ could generalize such contributions for arbitrary levels of $G$.

Alternative two-part formulation. Hoffmann and Chan [2012] reformulate Welford's twopart model (Equation (2.6)) to separate the effect of movement amplitude from that of ID, as

$$
M T=a^{\prime}+b^{\prime} \log _{2}(D)+c I D,
$$

where $a^{\prime}=a-c$, and $b^{\prime}=b-c$.

However, Hoffmann and Chan [2012] mention that the problem with Equations (2.6), and (2.8), is that taking logarithms of either $D$ or $W$ is invalid as these quantities are not unitless. Shoemaker et al. [2012] mention that Welford was aware of this problem and formulated normalizing constants $D_{0}$ and $W_{0}$, but these are not explicit. To avoid this problem, and based on the near-linear relation between $\log _{2}(D)$ and $\sqrt{D}$ for wide ranges of $D$ values, Hoffmann and Chan [2012] reformulate Equation (2.8), by replacing the $\log _{2}(D)$ term, by $\sqrt{D}$, such that

$$
M T=a^{\prime \prime}+b^{\prime \prime} \sqrt{D}+c I D,
$$

where the approximate values of $a^{\prime \prime}$, and $b^{\prime \prime}$ are given by the regression estimates of $\log _{2}(D)$ on $\sqrt{D}$ for the range of $D$ in the experiment, such that

$$
\begin{aligned}
a^{\prime \prime} & \approx a^{\prime}+b^{\prime} \beta_{1} \\
b^{\prime \prime} & \approx b^{\prime} \beta_{2} \\
\log _{2}(D) & \sim \mathcal{N}\left(\beta_{1}+\beta_{2} \sqrt{D}, \sigma^{2}\right) .
\end{aligned}
$$

These approximations allow prediction of the change in coefficients for regressions of the two-part Welford model [Hoffmann and Chan 2012]. The square-root of the distance had been previously shown to be linearly related to $M T$ on purely ballistic movements [Gan and Hoffmann 1988], therefore, under this model, the distance covering phase is assumed to be mostly ballistic, whereas the homing-in phase is assumed to follow to Fitts' ID [Hoffmann and Chan 2012]. 


\subsubsection{Fitts' Law formulations for 2-D static-target pointing tasks}

Fitts' Law is inherently 1-D, but in practice it is used on 2-D tasks, effectively ignoring the effects of both target angle $(\theta)$, and target shape on movement time. A 2-D target selection with a bivariate target (i.e., with different width $W$, and height $H$ ) is shown in Figure 2.1.

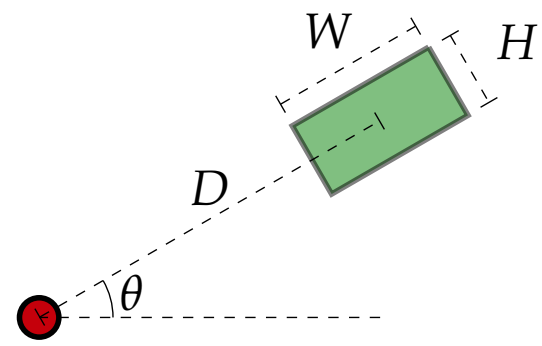

Figure 2.1. Two-dimensional task with a bivariate target. The red disc indicates the cursor; the green rectangle represents a target with width $W$, and height $H . D$ is the cursor-target distance, and $\theta$ is the target angle.

Nonetheless, several works have studied the effect of $\theta$ on MT, including the early work of Card et al. [1978] and Jagacinski and Monk [1985], the later work of Boritz et al. [1991], MacKenzie and Buxton [1992], and Whisenand and Emurian [1995; 1996; 1999], as well as the more recent work of Appert et al. [2008], Grossman and Balakrishnan [2004; 2005], Hancock and Booth [2004], Murata and Iwase [2001], Phillips and Triggs [2001], and Zhang et al. [2012]. Overall, it appears that MT is longer for diagonal targets, than for horizontal and vertical ones (the most notable exceptions are the results of Murata and Iwase [2001], Whisenand and Emurian [1999], and Zhang et al. [2012], who found that vertical targets were slower than diagonal targets).

To the author's knowledge, the only modification of Fitts' ID that exclusively models the effect of $\theta$, is that of Murata and Iwase [2001], which they derive empirically,

$$
I D_{3}=\log _{2}\left(\frac{D}{W}+1\right)+c \sin \theta,
$$

where $c$ is an empirically determined constant. A desirable characteristic of this model, is that it separates the effect of $\theta$ from the $\log _{2}$ term.

In addition to $\theta$, researchers have also studied targets of multiple dimensions and shapes. In these studies, the first category of extensions to Fitts' $I D$, which includes the $I D_{\text {min }}$ model by MacKenzie and Buxton [1992] and Hoffmann and Sheikh [1994], and the one-weight euclidean model by Accot and Zhai [2003], accounts for bivariate targets, but not explicitly for $\theta$. A similar yet more general category, which includes the models by Sheikh and Hoffmann [1994], Murata [1999], and Grossman and Balakrishnan [2005], allows modeling for arbitrary target shapes through the use of probabilistic models, but not explicitly for $\theta$. A third category, 
which includes the $I D_{W^{\prime}}$ model by MacKenzie and Buxton [1992], and the $I D_{\theta}$ model by Zhang et al. [2012] accounts for both bivariate targets and $\theta$, but cannot account for $\theta$ when targets are univariate (e.g., circles). Finally, the most relevant category for the current research allows modeling for $\theta$ and multiple target dimensions separately; two models in this category are presented below.

The model by Appert et al. [2008] introduces an overall angle effect as a cosine that is added within the log term of the $I D$,

$$
I D_{\theta}=\log _{2}\left(\frac{D}{W}+\frac{D}{H}+0.6 \cos (\theta) \frac{D}{\min (W, H)}+1\right),
$$

where $H$ is the target's height.

The weighted-euclidean ID formulation suggested by Grossman and Balakrishnan [2004] is an extension to the one-weight euclidean model of Accot and Zhai [2003] that allows modeling the angle effect separately for each of the target's dimensions (formulated and tested in 3-D, but reduced here to 2-D),

$$
I D_{\text {WtEuc } \theta}=\log _{2}\left(\sqrt{f_{W}(\theta)\left(\frac{D}{W}\right)^{2}+f_{H}(\theta)\left(\frac{D}{H}\right)^{2}}+1\right),
$$

where $f_{W}(\theta)$ and $f_{H}(\theta)$ are empirically determined weights per angle.

Equations (2.10)-(2.12) represent the contributions of $\theta$ in three distinct ways. Since the formulae are derived empirically, or by analogy, this raises the question of the true form of the contribution of $\theta$, which is probably why Grossman and Balakrishnan [2004] simply suggest to derive $f(\theta)$ empirically.

In terms of ease of use, Equations (2.10) and (2.11) are the simplest to solve, as a ubiquitous linear least-squares method may suffice, whereas Equation (2.12) requires a more specialized method, such as non-linear least-squares. In the case of the latter model, the fit in terms of $R^{2}$ may be artificially higher than the former due to the additional $2 \times \#(\theta)$ terms represented in $f_{W}(\theta)$, and $f_{H}(\theta)$. More importantly, the usage of $R^{2}$ may be inadequate to evaluate the goodness-of-fit of non-linear models [Spiess and Neumeyer 2010]. These last two points are important in the status quo of the Fitts literature, where model fit is customarily assessed using $R^{2}$.

Aside from the arbitrary shape models [Sheikh and Hoffmann 1994; Murata 1999; Grossman and Balakrishnan 2005], an important limitation of the aforementioned models is that they do not account for different target orientations.

\subsubsection{Fitts' Law formulations for 3-D static-target pointing tasks}

To the authors knowledge, the only work to formulate ID models specific for 3 -D is that of Grossman and Balakrishnan [2004]. These models, which are simple extensions to the 2-D 
models from the previous section, fail to account for all of the spatial parameters of a 3-D task, shown in Figure 2.2.

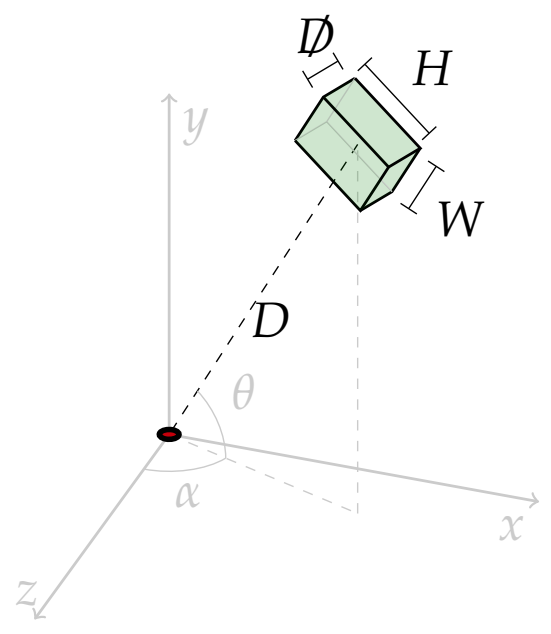

Figure 2.2. Three-dimensional trivariate target. The red disc indicates the cursor; the green rectangular cuboid represents a target with width $W$, height $H$, and depth $\not D . D$ is the cursor-target distance, $\alpha$ is the $z-x$ azimuth angle, and $\theta$ is the altitude angle.

For instance, the weighted-euclidean ID formulation was presented in Equation (2.12) as a 2-D formula, but in fact it was studied with trivariate targets placed in a horizontal 2-D plane. Its complete form is

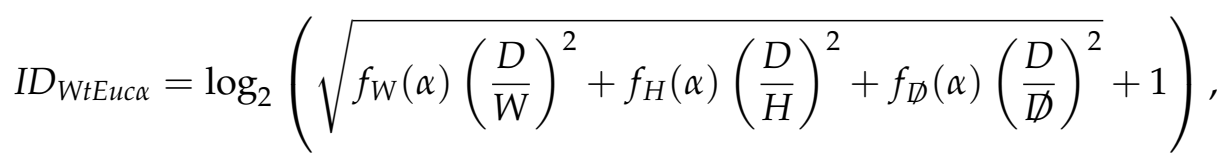

where $\not D$ is the target's depth, and $\alpha$ is the $z-x$ azimuth angle. Notice that this formulation does not account for the full angular position $(\alpha, \theta)$ of the target, nor does it account for different target orientations.

\subsubsection{Fitts' Law formulations for 1-D moving-target pointing tasks}

In comparison to static target selection, the research on Fitts' Law for 1-D and 2-D movingtarget selection is very scarce. After the early studies of Jagacinski et al. [1980] on the applicability of Fitts' Law for moving-targets in 1-D, only two studies have evaluated and extended Fitts' ID in moving-target selection tasks [Hoffmann 1991; Al Hajri et al. 2011].

Jagacinski et al. [1980] found that Fitts ID was a poor predictor of MT for moving targets with position control. Instead, they suggested an alternative formulation with analogous characteristics to two-part formulations, such as predicting longer MT for larger $D$ and smaller $W$, but also taking in account the interaction between $W$ and speed $(V)$. Their proposed formulation is 


$$
M T=a+b D+c(V+1)\left(\frac{1}{W}-1\right),
$$

where the $V+1$ term represents the $W \times V$ interaction, minimized for the widest target in their experimental design $\left(W=0.92^{\circ}\right)$ by subtracting 1 from $1 / W$ (since $\left.1 / 0.92 \approx 1\right)$. In Jagacinski's data, this model yielded a good fit in terms of $R^{2}=0.96$.

As discussed by Jagacinski and colleagues, the " -1 " term can be considered as a fourth parameter, e.g., $q$, thus a possible reformulation of Equation (2.14) is

$$
M T=a+b D+c(V+1) \frac{1}{W}-d(V+1),
$$

where $d=c q$. With this fourth parameter, the formulation becomes more general, and extensible to other experimental designs. Compared to the other formulations presented in this work, this one stands out for not including any logarithmic, or square-root terms.

In the Jagacinski study, trials were considered successful only after the cursor remained within the target for at least $350 \mathrm{~ms}$. This capture time duration $\left(T_{c}\right)$ was probably appropriate for Jagacinski's input device (a joystick), but may be different for other input methods, such as a mouse click, which takes about $200 \mathrm{~ms}$ for the average user according to the Keystroke-Level Model (KLM) [Card et al. 1980], or pointing on a touch screen, which theoretically entails a $0 \mathrm{~ms}$ capture time. To take the $T_{c}$ in consideration, Jagacinski et al. [1980] propose an alternative model,

$$
M T=a+b \log _{2}\left(\frac{2 D}{W}\right)+c \log _{2}\left(\frac{V}{W / T_{c}}+1\right) .
$$

Notice that this model simply reduces to Fitts' Law when $V=0$, or $T_{c}=0$ (e.g., in a touch screen). Unfortunately, the fit of this model on their data was low in terms of $R^{2}=0.71$, compared to the de facto standard in the Fitts literature, where $R^{2}>0.81$ [MacKenzie 1992, p. 101]. A third model was also proposed by Jagacinski and colleagues, but it contained the MT term on both sides of the equation and it did not, reportedly, result in a better fit in terms of $R^{2}$; therefore, it will not be taken in consideration.

Using a first-order control system, Hoffmann [1991] formally derived an ID for movingtarget selection closer in form to Fitts original ID,

$$
I D_{H}=\log _{2}\left(\frac{D \pm \frac{V}{K}}{\frac{W}{2}-\frac{V}{K}}\right),
$$

where $K$, described as the person's gain, is determined empirically, and the \pm on the numerator is determined by the direction of $V$ relative to the starting position: "+" for approaching targets, and "-" for distancing targets. ${ }^{2}$ Similarly to Welford et al. [1969], Hoffmann also suggested a two-part formulation for his own model,

\footnotetext{
${ }^{2}$ Distancing targets are those moving away from the cursor.
} 


$$
M T=a+b \log _{2}\left(D \pm \frac{V}{K}\right)+c \log _{2}\left(\frac{W}{2}-\frac{V}{K}\right) .
$$

Reusing the experimental data from Jagacinski et al. [1980], Hoffmann [1991] validated these two models empirically, yielding fits with $R^{2}$ values of 0.84 for Equation (2.17), and 0.94 for Equation (2.18). Interestingly, neither model, although theoretically sound, was able to surpass Jagacinski's empirical model (Equation (2.14)). The $R^{2}$ fit of the models in Equations (2.14) and (2.18) are practically equivalent, but the latter includes an additional term $(K)$ that also contributes to the coefficient of multiple determination.

Aside from the goodness-of-fit issues, Hoffmann's moving-target models require special considerations regarding the calculation and interpretation of the $K$ term that are not explicitly addressed in the literature. According to Hoffmann [1991], the coefficient $K$ serves to determine the critical speed $\left(V_{\text {crit }}\right)$ beyond which target capture is not possible,

$$
V_{\text {crit }}=\frac{W K}{2} \text {. }
$$

Hoffmann [1991] calculated $K$ in two distinct ways: using regression, and observing the $(V, W)$ condition in which capture occurred less than $\rho=50 \%$ of the time. The regression approach requires a specialized technique such as non-linear least-squares (which involves the additional issues explained at the end of Section 2.1.2.2) and yields values of $K$ whose domain is dependent on the experimental design. ${ }^{3}$ The alternative approach results in values of $K$ that are dependent on the percentage of successful captures $(\rho)$ per $(D, W, V)$ condition, thus, its precision depends on the span and resolution on the conditions in the experimental design, as well as the device-specific time of capture. Using either method, once $K$ is calculated, the set of experimental conditions $(D, W, V)$ that can be mathematically modeled by Equations (2.17) and (2.18) is restricted to

$$
\left\{(D, W, V) \mid \frac{V}{W / 2}<K\right\}
$$

for approaching targets, and

$$
\left\{(D, W, V) \mid \frac{V}{W / 2}<K \Longleftrightarrow \frac{V}{D}<K\right\}
$$

for distancing targets.

\subsubsection{Fitts' Law formulations for 2-D moving-target pointing tasks}

Al Hajri et al. [2011] also derived the $I D_{H}$ formulation (Equation (2.17)) by applying the human processor model [Card et al. 1980] to describe moving-target selection. Subsequently, they suggested three extensions for $I D_{H}$ by combining it with the $I D_{W^{\prime}}$ and $I D_{\min }$ formulations

\footnotetext{
${ }^{3}$ For approaching targets, $K>\frac{\max (V)}{\min (W / 2)}$; for distancing targets, $\left(\frac{\max (V)}{\min (W / 2, D)}<K\right) \oplus\left(\frac{\min (V)}{\max (W / 2, D)}>K\right)$. Otherwise, Equations (2.17) and (2.18) are not defined for all $(D, W, V)$ conditions.
} 
by MacKenzie and Buxton [1992], and the $I D_{W t E u c \theta}$ model (Equation (2.12)) by Grossman and Balakrishnan [2004], respectively. As discussed in Section 2.1.2.2, ID ${ }_{W t E u c \theta}$ is the most relevant among these three models, therefore only the extension $I D_{H W t E u c \theta}$ is considered,

$$
I D_{H W t E u c \theta}=\log _{2}\left(\sqrt{f_{W}(\theta)\left(\frac{D \pm \frac{V_{x}}{K}}{\frac{W}{2}-\frac{V_{x}}{K}}\right)^{2}+f_{H}(\theta)\left(\frac{D \pm \frac{V_{y}}{K}}{\frac{H}{2}-\frac{V_{y}}{K}}\right)^{2}}+1\right),
$$

where $V_{x}$ and $V_{y}$ are the magnitudes of the $x$ and $y$ components of the velocity, respectively.

Even though Equation (2.21) can model pointing at bivariate targets moving in 2-D, the studies of Al Hajri et al. [2011] only included conditions in which targets were moving directly towards or directly away from the cursor, even if the initial target angle $\theta \in[0,360)^{\circ}$.

\subsection{Subjective difficulty}

In addition to describing $M T$, ID has also been shown to be correlated to subjective difficulty [Delignières and Famose 1992; Slifkin and Grilli 2006; Grilli 2011; Chan and Hoffmann 2013]. There are two major types of subjective difficulty measurements in the literature:

1. Prospective, or estimated difficulty refers to ratings of subjective difficulty assessed without executing a task, e.g., in the works of Delignières [1990], Slifkin and Grilli [2006], and Grilli [2011]

2. Perceived difficulty refers to ratings of subjective difficulty assessed after executing a task, e.g., in the works of Delignières and Famose [1992], Shoemaker et al. [2012], and Chan and Hoffmann [2013].

There is some evidence that both measurements are correlated with each other [Delignières 1990; Grilli 2011], and with MT [Chan and Hoffmann 2013; Delignières 1993; Grilli 2011].

\subsubsection{The importance of the rating method}

The works of Delignières, described in his dissertation [1993], show that the best type of fit relating subjective difficulty to $I D$ is heavily influenced by the rating method. In his studies, the best fit between ID and subjective difficulty was linear when the latter was measured using a 15-point scale; when using a ratio-rating technique, the best fit was exponential.

\subsubsection{Subjective difficulty in moving-target selection}

To the author's knowledge, there is no study directly relating subjective difficulty and ID for moving-target selection. The closest work, is that of Famose et al. [1991], in which perceived difficulty ratings in a 15-point scale were shown to be negatively and linearly related to the scores in a dart throwing task on a moving-target. 


\subsection{Prediction of intention}

Predicting intended targets has been proposed as a solution to clutter and overlap in statictarget selection techniques. Current static-target prediction techniques are based on the trajectory and velocity profiles of the pointer [Lank et al. 2007; Noy 2001; Wonner et al. 2011; McGuffin and Balakrishnan 2005]. The peak accuracy rates for prediction using these techniques require a wide window of user input-at least $80 \%$ of the pointing movementbut some of them are intended to predict endpoints [Lank et al. 2007; Wonner et al. 2011], rather than intended targets [Noy 2001; McGuffin and Balakrishnan 2005]. These techniques, however, are not adapted for moving-target prediction, in particular due to the apparent dependency of the users' velocity profiles on the targets' movement [Carnahan and McFadyen 1996], and the fact that the peak hand velocity is attained upon target selection [Tresilian 2005].

\subsubsection{Scoring functions}

In the context of moving-target selection, the studies from de Haan et al. [2005] and Ortega [2013] demonstrated the feasibility of predicting intended targets in complex VR scenes using scoring functions. Overall, the stages of their algorithms can be described as follows,

1. Target filtering: A subset of the targets is chosen based on a given criterion.

2. Target scoring: Scores for targets that meet the criterion are incremented, and scores of targets that do not are decremented.

3. Target highlighting: The target with the highest score gets highlighted.

4. Target selection: The user completes the selection on the highlighted target by executing a button action, or continues moving the cursor until the intended target is highlighted.

In IntenSelect, the de Haan model [2005], the filtering criterion is given by an infinite cone with aperture $\beta_{\text {cone, }}$ apex located at the wand position, and orientation corresponding to wand vector $\mathbf{W}$. At each frame $t$, the wand-target angular distance $\alpha_{i}$ is calculated for each of the $I$ targets. Targets whose $\alpha_{i}<\beta_{\text {cone }}$, i.e., those inside the cone, get their scores increased, whereas the rest get their scores decreased. The score for each target $i$ is calculated following

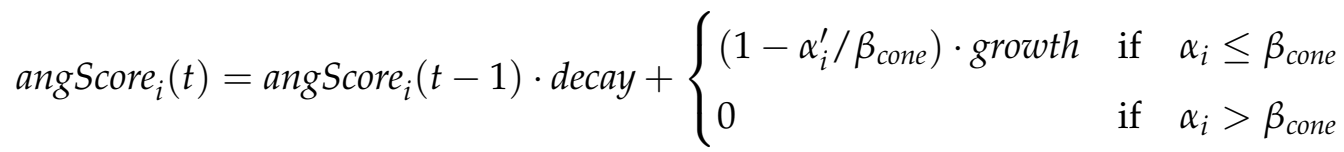

$$
\begin{aligned}
& \alpha_{i}^{\prime}=\arctan \left(\frac{D_{i, p e r p}}{D_{i, p r o j}^{k}}\right)
\end{aligned}
$$


where $D_{i, p e r p}$, and $D_{i, p r o j}$, correspond to the perpendicular and projected distances between the wand vector $\mathbf{W}$, and target $i$. Notice that $\alpha_{i}^{\prime}=\alpha_{i}$ when $k=1$; in the de Haan experiments, however, $k$ was heuristically selected as $4 / 5$, since $k=1$ led to distant targets being easier to reach than nearby ones. Further note that when decay $=0$, and growth $=1$, no score accumulation occurs, resulting in the flashlight, or conical selection technique [Bowman et al. 2004, 153-154]; de Haan et al. [2005] describe balancing these parameters to obtain "a comfortable response," but do not report the actual values.

In Hook, the Ortega model [2013], the filtering criterion is given by the wand-target distance $D_{i}$. At each frame $t$, the wand-target distance $D_{i}$ is calculated for each of the $I$ targets. Targets are then ordered ascendingly by $D$, their order given by $j_{i}=0, \cdots, I-1$. The score for each target $i$ is calculated following

$$
\begin{aligned}
& \operatorname{dScore}_{i}(t)=d \operatorname{Score}_{i}(t-1)+ \begin{cases}\left(N-j_{i}\right) \Delta t & \text { if } \quad j_{i}<N \\
-(\text { decay } \cdot N) \Delta t & \text { if } \quad j_{i} \geq N\end{cases} \\
& d \operatorname{Score}_{i}(t) \geq 0,
\end{aligned}
$$

where $N$ is an arbitrary number of closest targets, and decay is the rate with which scores decrease when a target $j_{i} \geq N$. In Ortega's studies with $I=100$ targets, $d \operatorname{dScore}_{i}(t)$ was used with $N=20$, and decay $=0.5$.

A disadvantage of de Haan's scoring function (2.22), compared to Ortega's scoring function, Equation (2.23), is that the former does not account for variable frame-rates. Scores are increased or decreased uniformly between frames regardless of the time difference $\Delta t$ between the frames. This is a major drawback, given that variable framerates are common in VR applications

These functions are easy to implement and their performance is enhanced as the user follows each target with the pointer; however, as it happens with some of the tasks in the present work, users may not always follow the intended target with their pointer. Additionally, there is no data on the predictive accuracy (i.e., the percentage of correctly predicted targets) of such functions, or how such accuracy is affected by the target distance-it is possible that users may have made their decision before starting their pointer movement, so the prediction could be done in advance.

\subsubsection{Gaze}

Knowing where a person is looking is considered an indicator of what is at the "top of the stack" of a cognitive process [Just and Carpenter 1976]. With respect to object manipulation, research has shown that gaze leads hand motions [Johansson et al. 2001]. Gaze is composed of head orientation and eye orientation relative to the head [Wilson et al. 2000]. 
In the context of target selection, eye gaze has proven to be beneficial in assisting users during static-target selection tasks, concurrently with more traditional input devices, such as mice [Zhai et al. 1999; Blanch and Ortega 2009]. Eye-trackers, however, are expensive and may be technically challenging to integrate in CAVE-like immersive vR systems [Murray et al. 2007], like the ones considered in the current work. Furthermore, this integration may be cumbersome due to the complex calibration procedures required or the cabling limitations of certain eye trackers [Murray et al. 2007]. Some modern solutions address these problems and allow eye-tracking in VR, but their adoption is still limited and costly. Head tracking, on the other hand, is readily available in most CAVE-like systems and has been successfully integrated in large-display [Nancel et al. 2013], video-conference [Stiefelhagen 2002], mobile [Spindler et al. 2012], surface [Francone and Nigay 2011], and floor-projected [Pierard et al. 2012] interactive systems.

In a series of studies based on video recordings, Stiefelhagen and colleagues demonstrated the potential of using head orientation to detect gaze and pointing. In a meeting scenario, Stiefelhagen and Zhu [2002] showed that, on average, head orientation represents $68.9 \%$ of the overall gaze direction, and could predict the observed person, among 3 people, with $88.7 \%$ accuracy. In a subsequent set of four meeting scenarios, once again with one observer and 3 possible observed people, the usage of head pose yielded an average accuracy of $72.9 \%$, which increased to $75.6 \%$ when combined with audio information that indicated who was speaking at each point in time Stiefelhagen [2002]. Finally, Nickel and Stiefelhagen [2003] found that head orientation was predictive of pointing direction, with a mean error angle of $22^{\circ}$, as well as intended target among eight targets, with $75 \%$ average accuracy. 


\section{CHAPTER 3}

\section{INTENTION IN UNDIRECTED 3-D MOVING-TARGET SELECTION}

Based on the premise that users form their intentions by minimizing their prospective effort, as described by $I D$, Chapter 1 introduced the hypothesis that intention can be predicted using ID in undirected selection tasks.

Unfortunately, as shown in Chapter 2, the existing ID measures formulated specifically for moving-target selection are not compatible with this PD-ID framework. The Hoffmann [1991] moving-target model, presented in Equation (2.17), and its 2-D extension by Al Hajri et al. [2011], presented in Equation (2.21), require the percentage of actual captures, which is unknown in prospective action, otherwise resulting in potentially harder and unreliable calculations.

On the other hand, a review of Jagacinski's studies [1980] reveals that even if Fitts' ID yielded poor correlations with MT for moving targets, the results were marginally better among conditions with equal target speeds. Additionally, low speed conditions resulted in slightly larger MT-ID correlations than high speed conditions. Mindful of these limitations, this Chapter evaluates the hypothesis that ID may be predictive of user intention in movingtarget selection tasks that have a single, relatively low $V$. Analogous to the way Jagacinksi's model [1980], Equation 2.14, uses $D$ and $W$ separately to predict $M T$, the predictive accuracy of $I D$ is compared to that of its separate $D$ and $W$ components.

The potential usefulness of $I D$ and $W$, however, relies on the assumption that the user is free to choose their intended target, in other words, it is limited to undirected tasks. Therefore, these task-specific measures are complemented with generalizable features that can also be used in directed tasks. Specifically, given their success at predicting user intention in directed tasks as reported in the related-work chapter, the proposed generalizable features consist of user-target states.

The contents of this chapter are based on papers published by the author and some of the committee members. Specifically, the $I D, D$, and $W$ analysis appeared on Casallas et al. [2013], and the generalizable feature extensions appeared on Casallas et al. [2014]. 


\subsection{Hypotheses}

The following hypotheses motivated the current study,

\subsubsection{Task-specific features for intention prediction in undirected moving-target selec- tion}

H1. ID can accurately predicting intention in undirected moving-target selection tasks.

\subsubsection{Generalizable features for intention prediction in undirected moving-target se- lection}

H2. Relative user-target features can accurately predict intention in undirected moving-target selection tasks.

H3. Combining task-specific, and generalizable features yields better predictive accuracy than using either separately.

\subsection{Pilot study}

Prior to the execution of the main experiment, a pilot study was conducted as a class project for the Machine Learning course (CS 573) at Iowa State University during the Spring of 2012, with classmates Ashwin S. Natarajan, and Keji Hu. The goal of the pilot study was to determine the best learning algorithm between Naïve Bayes [Mitchell 1997, 177-180], Neural Networks [Bishop 2006, 225-290], and C4.5 decision trees [Quinlan 1993], as well as the optimal time to measure the $D$, and $W$ features to predict the intended target.

Ten unpaid students participated in the experiment, which was deployed in METaL, a 3-surface CAVE-like vE. The experimental setup is depicted in Figure 3.1.

The results favored the usage of the 4.5 algorithm, which was second to Neural Networks in terms of accuracy but, contrary to the latter, produced easy-to-understand rules. Additionally, the results supported the usage of the $D$, and $W$ features measured at the beginning of each trial over features measured at one-third, and half of the total trial time. Due to restrictions of the Iowa State University Institutional Review Board (IRB) for in-class projects, the full extent of the results is not published.

\subsection{Methods}

\subsubsection{Participants}

Twenty-six unpaid participants, from the city of Chalon-sur-Saône, France aged 23 to 47, participated in the study. There were eighteen males and eight females; only two participants 


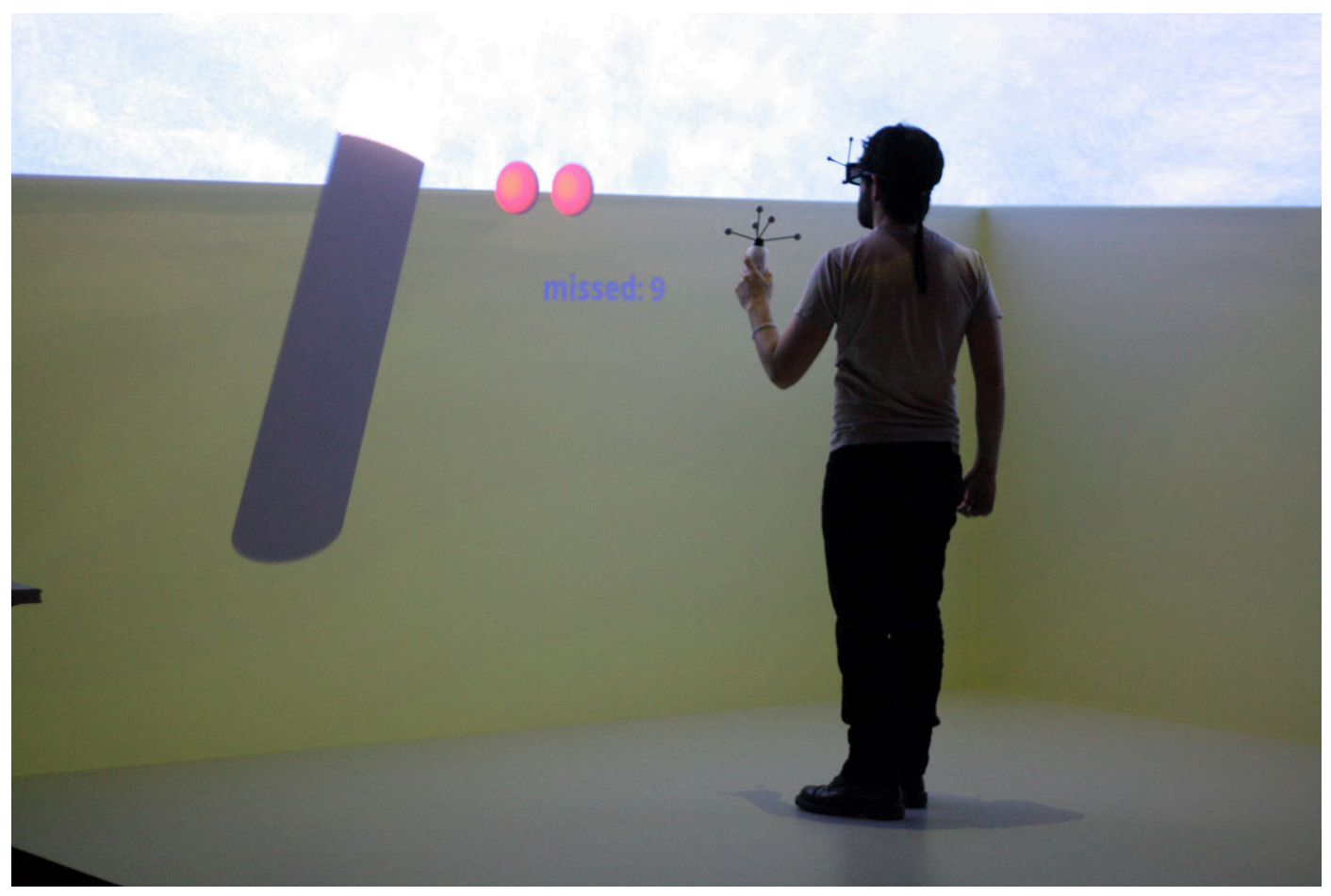

Figure 3.1. Experimental setup of the pilot study.

were left-handed.

\subsubsection{Apparatus}

The experiment was developed in VR Jugglua [Pavlik and Vance 2012], a Lua wrapper for vR Juggler and openscenegraph (OSG). The application was deployed in the MovE, a 4-surface CAvE-like virtual environment with three walls and a floor. The $3 \times 3 \times 2.67 \mathrm{~m}$ environment was projected using passive Infitec stereo [Jorke et al. 2008] at $1160 \times 1050$ pixels per face. Four infrared ART cameras tracked the pose (position, $P$, and orientation, $Q$ ) of the participant's head and wand, using reflective markers mounted on Infitec stereo glasses and an ART Flystick2, respectively. This allowed the participant to have an adequate 3-D perception and interact with the virtual world.

A $y$-up coordinate system was used, with its origin placed at ground level in the middle of the $\mathrm{VE}, z$ decreasing towards the front wall, and $x$ increasing towards the right wall.

\subsubsection{Procedure}

After filling a short survey, the participant was asked to enter the MOVE, face the front wall, and stay on a circular landmark $(r=0.25 \mathrm{~m})$ located in the middle of the vE $(0,0,0)$, while completing a series of target selection tasks. In each trial, the participant was presented with 
a horizontal array of virtual spheres of different sizes, starting in front of them and flying towards them in $z$. All of the spheres had the same texture, scaled accordingly to the sphere's size. The participant was instructed to touch each sphere by extending their arm only to reach the spheres; as opposed to wait for the spheres with their arm already extended. If a sphere was touched, or if it got $0.5 \mathrm{~m}$ past the participant's head in $z$, it disappeared. Each trial ended when the participant had touched all of the spheres, or when the remaining spheres got past their head.

Visual and auditory feedback were used to engage with the participant and indicate their performance. A virtual counter was placed at ground level, $5 \mathrm{~m}$ in front of the participant at $(0,0,-5)$, which would show the number of missed spheres during each block; the counter would be reset to zero at the beginning of each block of trials. When the participant hit a sphere, a spatialized sound, co-localized with the wand position, would be played; when the spheres got past the participant's head, a different spatialized sound, co-localized with the overall centroid of the remaining spheres, would be played. Compared to the pilot study, the recreated virtual world had enhanced depth cues, such as a grid floor, better lighting, and textures for both the terrain and the spheres, some of these differences can be identified by comparing Figures 3.1 and 3.2.

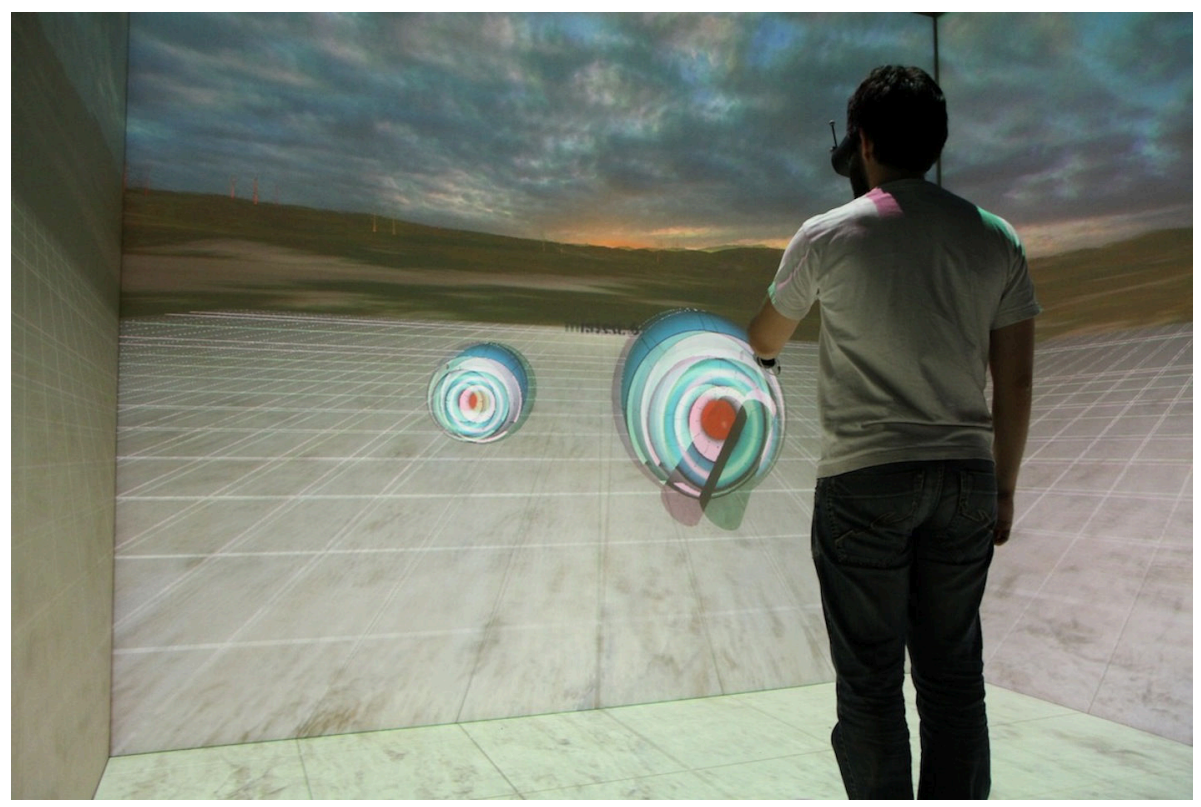

Figure 3.2. Experimental setup with an array two spheres

During each trial, at each application frame, the elapsed time $(t)$, head pose $\left(P_{h}, Q_{h}\right)$, wand pose $\left(P_{w}, Q_{w}\right)$, sphere positions $\left(P_{i}\right)$ and possible collisions between the wand and the spheres were recorded in a log file. 


\subsubsection{Design}

A within-subjects, factorial design was used, with three blocks of trials, each with a different number of conditions presented in a random order. In every trial, all of the spheres appeared $0.3 \mathrm{~m}$ below the participant's head and $5 \mathrm{~m}$ in front of them $\left(P_{i, y}=P_{h, y}-0.3, P_{i, z}=-5\right)$.

The first block each trial had only one sphere, moving at a constant speed of $2.5 \mathrm{~m} / \mathrm{s}$ in $z$. Factors were sphere radius $\left(r_{1} \in\{0.1,0.2\}\right)$ and sphere position (left: $P_{1, x}=0.5$, center: $P_{1, x}=0$, and right: $\left.P_{1, x}=0.5\right)$. Each of the six conditions was presented to the participant in a random order until completing five trials per condition (30 total). The first block was intended only for training, so that users could become familiar with the environment and the task.

After completing the first block, the number of spheres was increased to two and velocity was decremented to $1.5 \mathrm{~m} / \mathrm{s}$ in $z$. The spheres were positioned $0.5 \mathrm{~m}$ apart in $x$ but the pair could appear offset to the right $\left(P_{1, x}=-0.5, P_{2, x}=0\right)$, or left $\left(P_{1, x}=-0.5, P_{2, x}=0\right)$, or appear centered $\left(P_{1, x}=-0.25, P_{2, x}=0.25\right)$ with respect to the user (see Figure 3.3 ). Factors were sphere radius $\left(r_{i} \in\{0.1,0.2\}\right)$ and row position (left, center and right). Each of the 12 conditions was presented to the participant in a random order until completing five trials per condition (60 total).
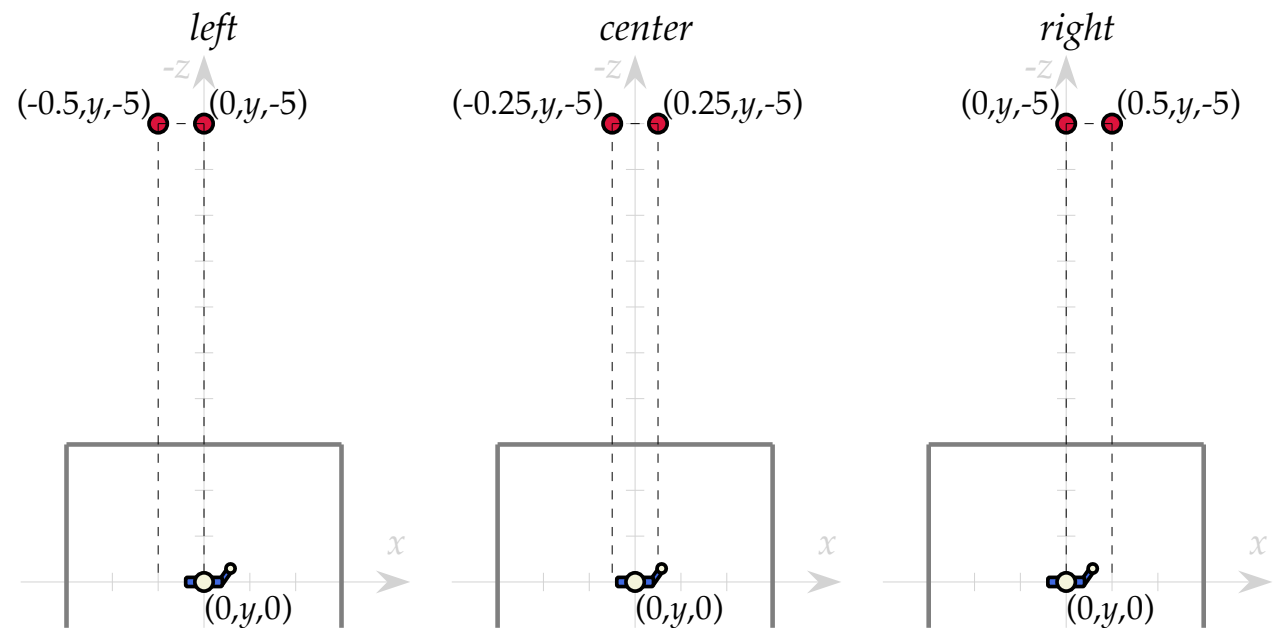

Figure 3.3. Possible row positions-left, center and right-with respect to the user in the twosphere block

In the last block, there were 40 trials with three spheres per row moving with the same velocity. Nevertheless, to simplify the presentation of predictive methods and results, the scope of the analyses covered in this chapter only include the 2-sphere case. Instead, the analysis of a more complex task with one, three, and six spheres, and different velocities is presented in Chapter 5. 


\subsubsection{Data integrity}

Since the goal was to predict intended targets, and not trial performance, trials in which a participant did not touch any sphere were discarded.

\subsubsection{Predictive methods}

Based on the results from the pilot study, all the feature-sets were evaluated using the J48 classifier, the Weka [Hall et al. 2009] open source implementation of $\mathrm{c} 4.5$. The algorithm is briefly described below.

$\mathrm{C}_{4} .5$ chooses its decision nodes recursively, based on the feature $(A)$ that yields the greatest information gain $(\mathcal{I})$, such that

$$
\mathcal{I}(S, A)=\mathcal{H}(S)-\mathcal{H}(S \mid A),
$$

where $\mathcal{H}(S)$ is the entropy of the training set $S$, given by

$$
\mathcal{H}(S)=-\sum_{i=1}^{I} p_{i} \log _{2} p_{i}
$$

where $p_{i}$ is the proportion of elements in $S$ that belong to class $i$, which corresponds in this experiment to the ratio of the number of trials in which sphere $s p h_{i}$ was chosen to the number of trials in the training set, i.e.,

$$
p_{i}=\frac{n_{i}}{\#(S)} \text {. }
$$

Finally, $\mathcal{H}(S \mid A)$ is the entropy of the training set $(S)$ split by the values of feature $A$, its value corresponds to

$$
\mathcal{H}(S \mid A)=\sum_{v \in \operatorname{Values}(A)} \frac{\#\left(S_{v}\right)}{\#(S)} \mathcal{H}\left(S_{v}\right),
$$

where $S_{v}$ corresponds to the subset obtained by splitting $S$ by the value $v$ of feature $A$. In addition to using $I$ to choose its decision nodes, the $\mathrm{C} 4.5$ algorithm uses additional rules to simplify the resulting tree and reduce overfitting. ${ }^{1}$

The advantage of $\mathbf{C 4 . 5}$, as stated in Section 3.2, is that it produces easy to interpret rules and a relatively high accuracy. In this study's scope, the decision trees allowed representation and analysis of the possible participant strategies to solve each task. To further avoid tree over-fitting to the experimental data, 10-fold cross validation was used on the generated tree models.

\footnotetext{
${ }^{1}$ The sole usage of $\mathcal{I}$ for decision-node selection is characteristic of the ID3 algorithm [Quinlan 1986], a predecessor of $\mathrm{C} 4 \cdot 5$.
} 
Due to the undirected nature of the studied task, participants may exhibit an overall preference for the left or right sphere. Therefore, the results obtained from $\mathrm{C} 4.5$ are compared to a zero-rule classifier that always predicts the most frequently chosen sphere. Such a classifier is equivalent to a 1-node decision tree generated from an empty feature-set $(\varnothing)$.

\subsubsection{Model comparison}

Models are compared based on their accuracy (acc), or percentage of correct predictions. To give a rough sense of the uncertainty around the accuracy $(a c c)$ measurements of each model, the $95 \% \mathrm{CI}$ of acc is approximated using

$$
a c c \pm z .95 \sqrt{\frac{a c c(1-a c c)}{\text { numTrials }}}
$$

where $z_{.95} \approx 1.96$ is the $97.5^{\text {th }}$ percentile of the normal distribution. According to Mitchell [1997, p. 141], this 95\% CI approximation using the normal distribution is adequate when numTrials $\geq 30$, which is the case of this study.

In cases where the confidence interval comparison is not sufficient to elucidate "significant" model differences, an additional test is warranted to gauge the uncertainty in the difference of accuracies. Based on Equation (5.13) from [Mitchell 1997, p. 144] the 95\% CI for the difference between the accuracies of two classifiers is given by

$$
\left(a c c_{a}-a c c_{b}\right) \pm z .95 \sqrt{\frac{a c c_{a}\left(1-a c c_{a}\right)}{\text { numTrials }}+\frac{a c c_{b}\left(1-a c c_{b}\right)}{\text { numTrials }}}
$$

Note, however, that all feature-sets are tested on the same trials, thus, the confidence intervals given by Equation (3.6) may be too conservative [Mitchell 1997, p. 144].

\subsection{Task-specific feature analysis}

Based on the initial wand position $\left(P_{w}\right)$, sphere diameter $\left(W_{1}, W_{2}\right)$ and initial sphere position $\left(P_{1}, P_{2}\right)$, different values were calculated, including wand-sphere distances,

$$
\begin{aligned}
& D_{1}=\left|P_{w}-P_{1}\right|, \\
& D_{2}=\left|P_{w}-P_{2}\right|,
\end{aligned}
$$

wand-sphere indices of difficulty using Shannon's formulation (Equation (2.5)),

$$
\begin{aligned}
& I D_{1}=\log _{2}\left(\frac{D_{1}}{W_{1}}+1\right), \\
& I D_{2}=\log _{2}\left(\frac{D_{2}}{W_{2}}+1\right),
\end{aligned}
$$

inter-sphere distance, 


$$
D_{\text {sph }}=\left|P_{2}-P_{1}\right|
$$

inter-sphere indices of difficulty,

$$
\begin{gathered}
I D_{1,2}=\log _{2}\left(\frac{D_{s p h}}{W_{2}}+1\right) \\
I D_{2,1}=I D_{1,2}=\log _{2}\left(\frac{D_{s p h}}{W_{1}}+1\right),
\end{gathered}
$$

and total indices of difficulty

$$
\begin{aligned}
& I D_{T 1}=I D_{1}+I D_{1,2}, \\
& I D_{T 2}=I D_{2}+I D_{2,1},
\end{aligned}
$$

Using the C4.5 algorithm, feature-sets $\left\{I D_{T 1}, I D_{T 2}\right\},\left\{I D_{1,2}, I D_{2,1}\right\},\left\{I D_{1}, I D_{2}\right\}$ and $\left\{D_{1}, D_{2}, r_{1}, r_{2}\right\}$ were evaluated to predict the first selected sphere.

\subsubsection{Results}

Participants showed an overall preference for the right sphere $(\sim 64 \%)$. Thus, as shown in the last row of Table 3.1, the decision tree generated using the zero-rule approach always predicted $\mathrm{sph}_{2}$ as the selected sphere with an accuracy of $63.81 \%, 95 \% \mathrm{CI}[61.41 \%, 66.21 \%]$.

Decision trees generated with the $\mathrm{c} 4.5$ algorithm from feature-sets 1-4 (see Table 3.1) yielded approximately $71 \% \pm 2.26 \%$ accuracy on predicting the selected sphere, with a $95 \%$ confidence level, which is significantly better than both chance $(50 \%)$, and the aforementioned zero-rule predictor $(64 \% \pm 2.4 \%)$. Even though all of the feature-sets yielded similar accuracies, the generated tree for feature-set 1 was more complex than those generated for feature-sets 2-4, making it less practical and perhaps over-fitted to the data [Mitchell 1997], especially considering that its 4 non-leaf nodes were generated from only 2 attributes.

Table 3.1. Accuracy and 95\% confidence intervals for the evaluated feature-sets

\begin{tabular}{cccccc} 
& Feature-set & Size & Leaves & acc & $95 \% \mathrm{CI}$ \\
\hline 1 & $I D_{T 1}, I D_{T 2}$ & 9 & 5 & $70.56 \%$ & {$[68.28 \%, 72.83 \%]$} \\
2 & $I D_{1,2}, I D_{2,1}$ & 5 & 3 & $71.21 \%$ & {$[68.95 \%, 73.47 \%]$} \\
3 & $I D_{1}, I D_{2}$ & 5 & 3 & $70.95 \%$ & {$[68.68 \%, 73.21 \%]$} \\
4 & $D_{1}, D_{2}, r_{1}, r_{2}$ & 5 & 3 & $71.21 \%$ & {$[68.95 \%, 73.47 \%]$} \\
5 & $\varnothing$ & 1 & 1 & $63.81 \%$ & {$[61.41 \%, 66.21 \%]$}
\end{tabular}

Interestingly, the fact that feature-sets 2 and 4 had the same accuracy, 95\% CI, and a similar tree configuration (3 leaves out of 5 nodes) implies that they are equivalent. This may seem surprising, given that the only relevant factors in the inter-sphere indices of difficulty $\left(I D_{1,2}\right.$, 
$\left.I D_{2,1}\right)$ which compose feature-set 2 , are sphere diameters $\left(W_{1}, W_{2}\right){ }^{2}$, whereas feature-set 4 is composed not only of sphere radii $\left(r_{1}, r_{2}\right)$, but also of wand-sphere distances $\left(D_{1}, D_{2}\right)$. A closer look at the generated decision tree for feature-set 4 (Figure 3.4), however, reveals that the resulting tree included only sphere radii; wand-sphere distances $\left(D_{1}, D_{2}\right)$ were probably ignored by the 4.5 algorithm on the basis of low information gain. Thus, it is reasonable to conjecture that the radii provide an equivalent information gain to feature-sets 2 and 3 , since their generated trees had similar configurations and yielded an equivalent accuracy.

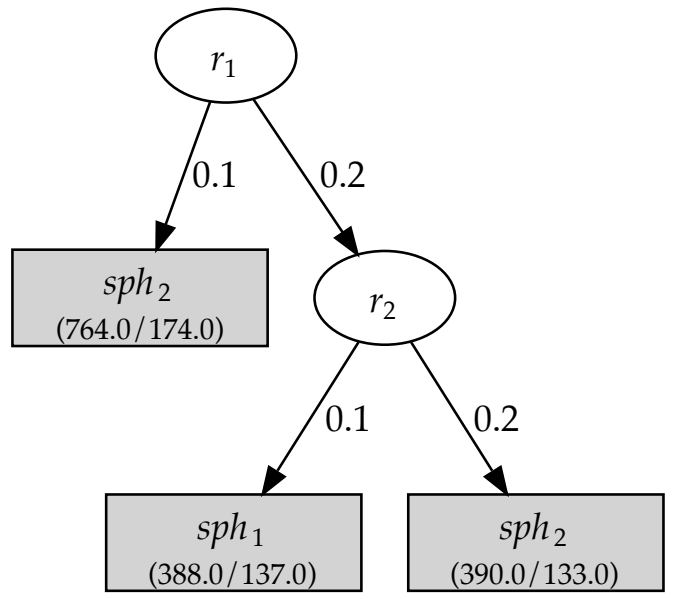

Figure 3.4. Decision tree for feature-set 4, suggesting that participants based their decisions only on sphere size, with a preference for the right sphere. Leaves represent prediction outcomes $\left(\mathrm{sph}_{1}\right.$ is the left sphere, and $\mathrm{sph}_{2}$ is the right sphere), while the other nodes represent tested attributes $\left(r_{1}\right.$ or $\left.r_{2}\right)$. The numbers in parenthesis within the leaves represent the total number of instances that fall into that leaf, over the number of incorrectly predicted instances among these instances.

The overall tendency for choosing the right sphere $\left(s p h_{2}\right)$ first is likely due to the majority of the participants being right-handed; unfortunately, there weren't enough left-handed participants to evaluate the effects of handedness on the generated models. According to the decision tree generated from feature-set 4 presented in Figure 3.4, participants would only choose the left sphere $\left(s p h_{1}\right)$ first if the right one $\left(s p h_{2}\right)$ was smaller; $s p h_{2}$ would be selected first if its radius was greater or equal than that of $s p h_{1}$.

\subsection{Generalizable feature analysis}

At each frame, measurements that could relate the target positions to the participant's head pose $\left(P_{h}, Q_{h}\right)$, and wand position $\left(P_{w}\right)$ were calculated. Subsequently, these measurements

\footnotetext{
${ }^{2}$ Inter-sphere distances are equal for all of the trials $\left(D_{\text {sph }}=0.5\right)$, annulling their influence on $I D_{1,2}$ and $I D_{2,1}$ and, thus, on feature-set 2, see Equations (3.12) and (3.13).
} 
were averaged in a time window, and different feature-sets were evaluated to predict the intended sphere.

\subsubsection{Relative user-target features}

First, the head-sphere vectors

$$
\mathbf{P}_{i h}=P_{i}-P_{h},
$$

are calculated, where $P_{i}$ corresponds to the absolute position of sphere $i \in\{1,2\}$.

Subsequently, the dot products between the normalized head $(\mathbf{H})$, and $\mathbf{P}_{i h}$ vectors

$$
\operatorname{dot}_{i}=\hat{\mathbf{H}} \cdot \hat{\mathbf{P}}_{i h} .
$$

are calculated. The head-target dot product, $d_{0} t_{i}$, has the advantage of being an easy to interpret, normalized scalar: the closer dot $_{i}$ gets to 1 , the more the user's head orientation is aligned with $s p h_{i}$. Since the spheres do not overlap in the user's field of view, the dot-product difference

$$
\Delta d o t=d o t_{1}-d o t_{2}
$$

is calculated. This quantity serves to determine the relative pose of the user's head with respect to the spheres. The closer $\Delta$ dot gets to 1, the more the user's head is aligned with $s p h_{1}$; the closer the quantity gets to -1 , the more the user's head is aligned with $s p h_{2}$; a value of 0 implies that the user's head is oriented right in the middle of both spheres.

Finally, the difference between wand-sphere distances

$$
\Delta D=D_{1}-D_{2}
$$

is calculated. Where $D_{1}$, and $D_{2}$ are the wand-sphere distances given by Equations (3.7) and (3.8). Similar to $\Delta d o t, \Delta D$ serves to determine the relative position of the user's wand with respect to the spheres. A positive $\Delta D$ implies that the wand is farther from $s p h_{1}$; a negative quantity implies that the wand is farther from $s p h_{2} ; 0$ implies that the wand is equidistant from both spheres.

\subsubsection{Distance score feature}

To validate the usefulness of the proposed user-target features, their predictive accuracy is compared to the distance scoring function proposed by Ortega [2013], presented in Equation (2.23). Since the experimental environment was composed of two targets, $N=1$ is chosen, such that only the closest target's score is increased. Compared to the decay $=0.5$ value used by Ortega, a higher decay $=0.9$ value is chosen to account for the fact that most of the movement happened late in each trial; a low decay rate would not have permitted to revert 
the possible target-score accumulation that occurs while participants are waiting for targets to become reachable. The resulting scoring function for each target $i$ at frame $t$ is therefore

$$
\begin{aligned}
& \operatorname{dScore}_{i}(t)=d \operatorname{Score}_{i}(t-1)+\left\{\begin{array}{lll}
\left(1-j_{i}\right) \Delta t & \text { if } & j_{i}<1 \\
-0.9 \Delta t & \text { if } & j_{i} \geq 1
\end{array}\right. \\
& \text { dScore }_{i}(t) \geq 0,
\end{aligned}
$$

where $j_{i}=0, \cdots, I-1$ is the ascending target order based on the wand-target distance $D_{i}$.

\subsubsection{Time-window selection}

To cope with issues of instability and inaccuracy that affect human movement [Shadmehr et al. 2010], the values of $\Delta d o t$ and $\Delta D$ are averaged in a time window, rather than using their instant values.

In interactive usage contexts, both the feature averaging and the scoring function start running upon user activation. The present analysis, however, is done post hoc, so the functions are applied to the data during a graphically determined time window. Ideally, the time window would start before the beginning of the reaching action, while the user is specifying their intentions and actions [Norman 2002], and end before the target is reached. In the scope of this study, the $\Delta d o t$ profile was analyzed graphically over time, to determine an appropriate window heuristically, as shown in Figure 3.5. Other possible approaches are discussed in the future work section.

Because there is no time between trials, the starting non-zero $\Delta$ dot values in Figure 3.5 are likely due to participants fixating the last sphere they touched on the previous trial. The subsequent convergence towards zero, between $0 \mathrm{~s}$ and $1 \mathrm{~s}$ suggests that their gaze is shared between both spheres, probably while making their decision. After $1 \mathrm{~s}, \Delta$ dot starts diverging again, suggesting that participants' heads are oriented towards one of the two spheres. If this is the case, the increased divergence could be related to the increased separation of the spheres in the participant's field of view, as they get closer to them. Furthermore, after $1 \mathrm{~s}$, the red- and blue-colored selection labels of $s p h_{1}$, and $s p h_{2}$, are more clearly clustered above and below zero, respectively.

This graphical evidence suggests that roughly $1 \mathrm{~s}$, and $1.5 \mathrm{~s}$ are good start, and end times for the window. These times roughly correspond to $42.5 \%$ and $63.8 \%$ of the $5^{\text {th }}$ percentile of the selection times $(2.35 \mathrm{~s})$. Within this window, both the mean dot product difference $(\overline{\Delta d o t})$ and the mean wand-target distance $(\overline{\Delta D})$ are calculated. 


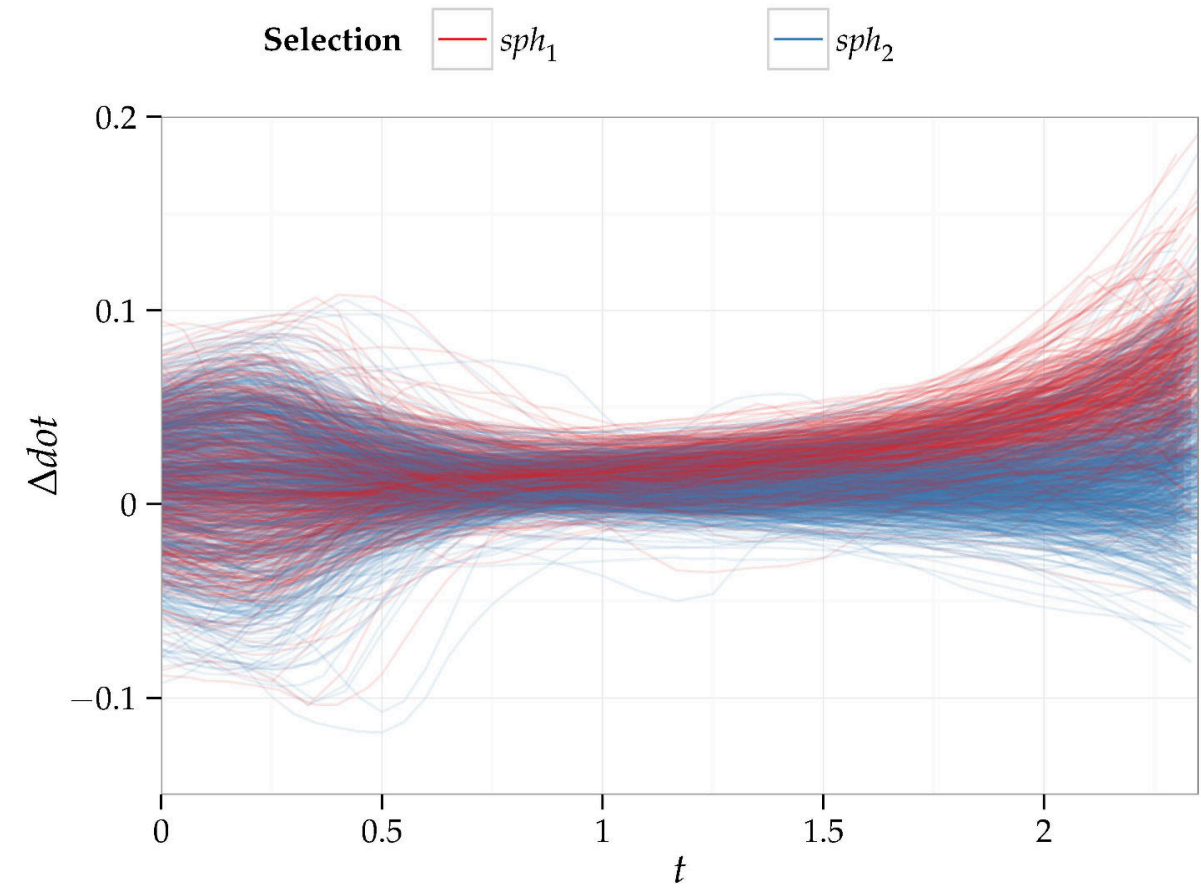

Figure 3.5. $\Delta$ dot vs. time. Each line corresponds to a trial, colored according to the selected sphere. The plot has been trimmed to the $5^{\text {th }}$ percentile of the selection times (2.35s). 


\subsubsection{Evaluation}

Generalizable user-target feature-sets $\{\overline{\Delta d o t}\},\{\overline{\Delta D}\},\{\overline{\Delta d o t}, \overline{\Delta D}\}$, and $\{\overline{\Delta d o t}$, as well as their combinations with the sphere radii, $\left\{\overline{\Delta d o t}, r_{1}, r_{2}\right\},\left\{\overline{\Delta D}, r_{1}, r_{2}\right\}$, and $\left., \overline{\Delta D}, r_{1}, r_{2}\right\}$ are evaluated using the 4.5 classifier to predict the first sphere selected by the user $\left(s p h_{i}\right)$.

Models generated from generalizable user-target features, $\overline{\Delta d o t}$ and $\overline{\Delta D}$, are compared to the scoring classifier bestDRank, which always predicts the chosen sphere as the one with the best $d$ Score, given by Equation (3.20).

In the case of feature-sets combining generalizable features, $\overline{\Delta d o t}$ and $\overline{\Delta D}$, and task-specific features, $r_{1}$ and $r_{2}$, the baseline classifier is the decision tree generated from the best feature-set from Section 3.4, i.e., $\left\{r_{1}, r_{2}\right\}$.

The performance of bestDRank is simply evaluated by calculating its predictive accuracy, i.e. the ratio of correct predictions to the number of trials. The performance of the remaining features is evaluated based on the 10-fold cross validation accuracy of the decision-trees generated from each feature-set.

\subsubsection{Results and discussion}

\subsubsection{Generalizable user-target features}

As shown in Table 3.2, all feature-sets performed better than chance and a zero-rule predictor. On average, all of the proposed feature-sets performed better than the bestDRank baseline classifier. Among the individual features, $\overline{\Delta D}$ yielded less average accuracy than $\overline{\Delta d o t}$ with a more complex tree, making it less practical and perhaps over-fitted to the data [Mitchell 1997, p. 67]. Furthermore, since the $\overline{\Delta D}$ accuracy was only marginally higher than that of the bestDRank baseline, no further analyses on the former are carried out.

Table 3.2. Tree size, number of leaves, accuracy and 95\% confidence intervals for the evaluated generalizable moving-target feature-sets

\begin{tabular}{ccccc} 
Feature-set & Size & Leaves & acc & $95 \%$ CI \\
\hline bestDRank & 1 & 1 & $68.09 \%$ & {$[65.77 \%, 70.42 \%]$} \\
$\overline{\Delta d o t}$ & 3 & 2 & $70.69 \%$ & {$[68.42 \%, 72.96 \%]$} \\
$\overline{\Delta D}$ & 5 & 3 & $68.42 \%$ & {$[66.1 \%, 70.74 \%]$} \\
$\overline{\Delta d o t}, \overline{\Delta D}$ & 11 & 6 & $71.73 \%$ & {$[69.48 \%, 73.97 \%]$}
\end{tabular}

Given that their $95 \%$ CI overlap, the difference between accuracies of bestDRank, $\overline{\Delta d o t}$, and $\overline{\Delta d o t}, \overline{\Delta D}$ is calculated, as well as the $95 \% \mathrm{CI}$ of this difference. Results are presented in Table 3.3.

The fact that feature-set $\{\overline{\Delta d o t}, \overline{\Delta D}\}$ yielded the greatest average accuracy, which was significantly better than both the baseline bestDRank and feature $\overline{\Delta D}$ confirms the value of using head-target and wand-target relative features to predict intention in moving-target 
Table 3.3. Accuracy difference and $95 \%$ confidence intervals for the evaluated generalizable moving-target feature-sets. Asterisks $\left(^{*}\right)$ denote a significant difference $(\alpha=0.05)$.

\begin{tabular}{cccc} 
Feature-set $_{a}$ & Feature-set $_{b}$ & $\Delta a c c$ & $95 \% \mathrm{CI}$ \\
\hline bestDRank & $\overline{\Delta d o t}$ & $-2.59 \%$ & {$[-5.96 \%, 0.67 \%]$} \\
bestDRank $^{\overline{\Delta d o t}}$ & $\overline{\Delta d o t}, \overline{\Delta D}$ & $-3.63 \%$ & {$[-7.01 \%,-0.41 \%]^{*}$} \\
$\overline{\Delta D}$ & $\overline{\Delta d o t}, \overline{\Delta D}$ & $-1.04 \%$ & {$[-4.33 \%, 2.2 \%]$} \\
$\overline{\Delta d o t}, \overline{\Delta D}$ & $-3.31 \%$ & {$[-6.68 \%,-0.08 \%]^{*}$}
\end{tabular}

selection. As previously stated, this is likely due to the inherent visuomotor nature of the moving-target selection tasks, where users need to fixate on the chosen target while moving their hands towards them. This model is presented in Figure 3.6.

Due to the task and evaluation differences with previous work on intention prediction in target selection, the results are not directly comparable to the latter, but suggest the potential of the presented approach. The time-window limits are likely to change according to the task (e.g., if the user has to search for their intended target in a cluttered environment), but it may be possible to detect patterns similar to Figure 3.5 when the intended target is fixated upon, which is more efficient than previous approaches that require large portions of the entire hand trajectory as predictive inputs [McGuffin and Balakrishnan 2005; Lank et al. 2007; Wonner et al. 2011].

Furthermore, in other tasks the generated tree nodes will likely have different split values than those presented in Figure 3.6. It is possible, however, that the split values for trees in other binary selection tasks will also be close to zero.

Finally, using a single relative head-target parameter, such as $\overline{\Delta d o t}$, and a single wand-target relative parameter, such as $\overline{\Delta D}$, may not be useful or viable in tasks with more and differently positioned targets. A solution could be to create similar features for every possible pair of targets.

\subsubsection{Combined task-specific and generalizable features}

Tables 3.4 and 3.5 show that Feature-set $\left\{\overline{\Delta d o t}, \overline{\Delta D}, r_{1}, r_{2}\right\}$ performed significantly better than all of the other feature-sets, surpassing the best task-specific feature-set, $\left\{r_{1}, r_{2}\right\}$, by almost $7 \%$, and the best generalizable feature-set, $\overline{\Delta d o t}, \overline{\Delta D}$, by about $6 \%$. Unfortunately, the generated tree was too big ( 21 nodes) to fit in this paper.

Surprisingly, and contrary to the results from the previous section, combining the $\overline{\Delta d o t}$ relative feature with the sphere radii $\left(r_{1}, r_{2}\right)$ did not yield better accuracy than feature-set $\left\{\overline{\Delta D}, r_{1}, r_{2}\right\}$.

The fact that feature-set $\left\{\overline{\Delta D}, r_{1}, r_{2}\right\}$ performed marginally better than the baseline, $\left\{r_{1}, r_{2}\right\}$, suggests that a function of target size and distance, albeit different from $I D$, can adequately predict the selected sphere in this type of task. In the results from Section 3.4, however, the 


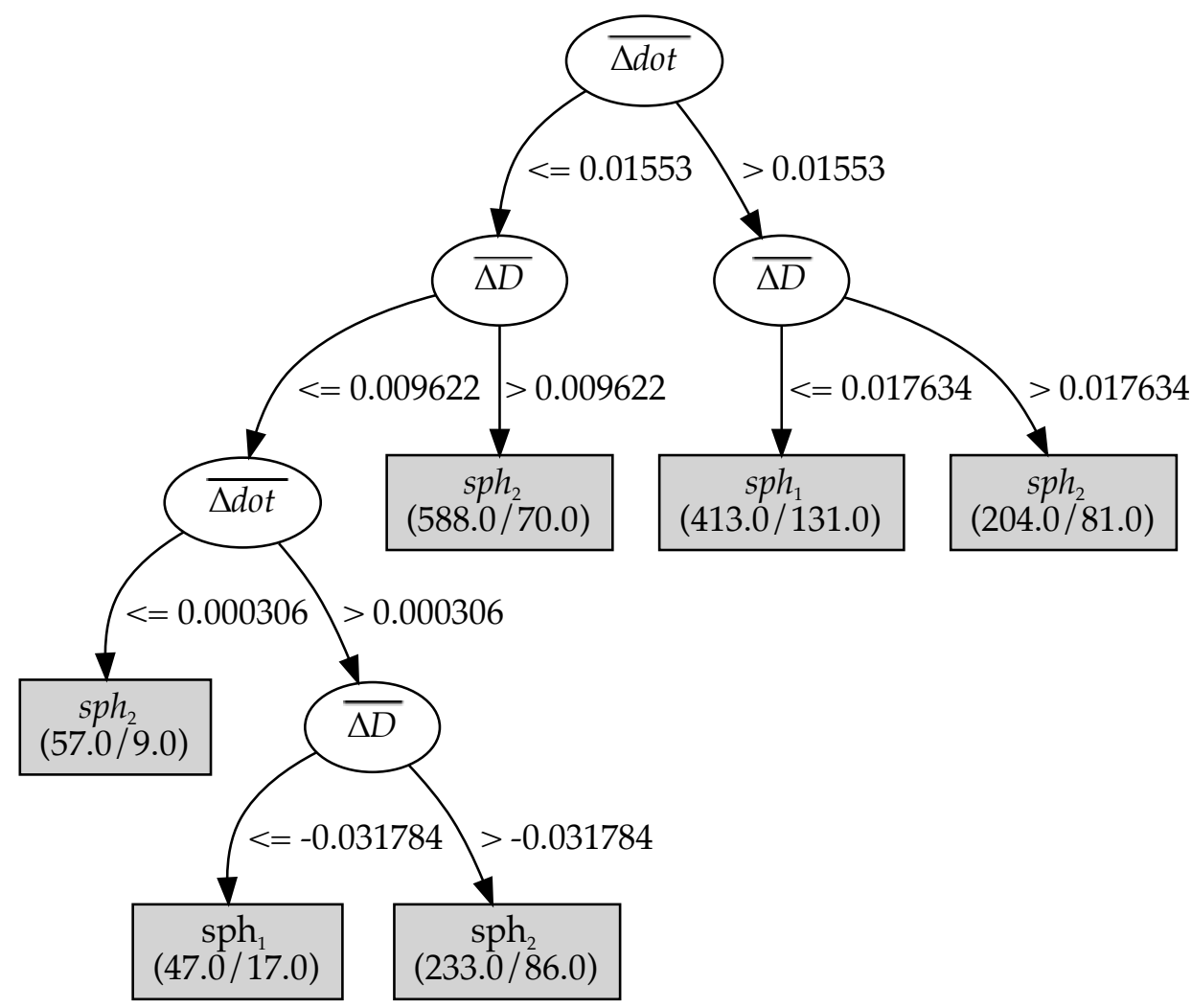

Figure 3.6. Generated decision tree for feature-set $\{\overline{\Delta d o t}, \overline{\Delta D}\}$. The numbers in parenthesis within the leaves represent the total number of instances that fall into that leaf, over the number of incorrectly predicted instances among these instances. 
distance $D_{0}$-measured at the beginning of each trial—was deemed to yield less information gain than the sphere radii. The apparent increase in information gain by integrating $\overline{\Delta D}$, observed in the present work, reflects a correlation between wand and object position, as previously suggested by Ortega [2013], but only after a certain preparation time [Nieuwenhuizen et al. 2009].

Table 3.4. Tree size, number of leaves, accuracy and 95\% confidence intervals for the evaluated target-based feature-sets.

\begin{tabular}{ccccc} 
Feature-set & Size & Leaves & $a c c$ & $95 \%$ CI \\
\hline$r_{1}, r_{2}$ & 5 & 3 & $71.21 \%$ & {$[68.95 \%, 73.47 \%]$} \\
$\overline{\Delta d o t}, r_{1}, r_{2}$ & 7 & 4 & $73.35 \%$ & {$[71.14 \%, 75.55 \%]$} \\
$\overline{\Delta D}, r_{1}, r_{2}$ & 27 & 14 & $74.19 \%$ & {$[72.01 \%, 76.37 \%]$} \\
$\overline{\Delta d o t}, \overline{\Delta D}, r_{1}, r_{2}$ & 21 & 11 & $78.02 \%$ & {$[75.95 \%, 80.08 \%]$}
\end{tabular}

Table 3.5. Accuracy difference and $95 \%$ confidence intervals for the target-based feature-sets. Asterisks $\left(^{*}\right)$ denote a significant difference $(\alpha=0.05)$, dots (.) denote a marginal difference $(\alpha=0.1)$.

\begin{tabular}{cccc} 
Feature-set $_{a}$ & Feature-set $_{b}$ & $\Delta a c c$ & $95 \%$ CI \\
\hline$r_{1}, r_{2}$ & $\overline{\Delta d o t}, r_{1}, r_{2}$ & $-2.14 \%$ & {$[-5.43 \%, 1.04 \%]$} \\
$r_{1}, r_{2}$ & $\overline{\Delta D}, r_{1}, r_{2}$ & $-2.98 \%$ & {$[-6.28 \%, 0.16 \%]$.} \\
$\overline{\Delta d o t}, r_{1}, r_{2}$ & $\overline{\Delta D}, r_{1}, r_{2}$ & $-0.84 \%$ & {$[-4.05 \%, 2.32 \%]$} \\
$\overline{\Delta D}, r_{1}, r_{2}$ & $\overline{\Delta d o t}, \overline{\Delta D}, r_{1}, r_{2}$ & $-3.83 \%$ & {$[-7.04 \%,-0.85 \%] *$}
\end{tabular}

\subsection{Discussion}

Results are summarized according to the study hypotheses, each followed by a discussion.

\subsubsection{Task-specific features for intention prediction in undirected moving-target selec- tion}

Considering that task-specific decision trees were built based only on the initial position of the user's wand and the initial size and position of the spheres, predictions bore a relatively high accuracy, compared to both chance and a zero-rule predictor. It is likely, however, that the accuracy will decrease if the number of targets is increased, but it is expected that the accuracy will still be better than chance and a zero-rule predictor.

H1. ID can accurately predicting intention in undirected moving-target selection tasks. Partially supported. 
Even though $I D$ served as a good task-specific feature for predicting the intended moving target, using solely sphere radii $\left(r_{1}, r_{2}\right)$ yielded an equivalent accuracy $(\sim 72 \%)$. Recall that all of the evaluated ID measures, presented in Equations (3.9)-(3.15), are based on target radii. This suggests that target size yields more information gain than the initial target distance, and indicates a very basic strategy from the users in which distance does not play an important role for choosing targets.

This may be due to the fact that the spheres get closer to the user throughout each trial, eventually annulling the $z$ component of the target's distance Additionally, since there was some waiting time before the targets were reachable, it is possible that users prepared the starting horizontal position of their wands prior to executing the pointing task, even if instructed otherwise.

\subsubsection{Generalizable features for intention prediction in undirected moving-target se- lection}

H2. Relative user-target features can accurately predict intention in undirected moving-target selection tasks. Supported.

The relative head-target and wand-target features, $\overline{\Delta d o t}$ and $\overline{\Delta D}$, respectively, proved successful in predicting intended targets in the studied undirected moving-target selection tasks. Combined, the features yielded a $\sim 72 \%$ accuracy on predicting intended targets, which was significantly better than the isolated $\overline{\Delta D}$ feature $(\Delta a c c=3.31 \%)$, and the Ortega [2013] scoring function $(\triangle a c c=3.63 \%)$.

The relative head-target feature, $\Delta d o t$, proved to be useful not only for prediction, but also for establishing the adequate time window. Currently, the window is established empirically, from the $\Delta$ dot vs. $t$ plot (Figure 3.5).

H3. Combining task-specific, and generalizable features yields better predictive accuracy than using either separately. Supported.

The integration of features $\overline{\Delta d o t}$, and $\overline{\Delta D}$, with $r_{1}$ and $r_{2}$, improved the predictive accuracy of intended targets in undirected moving-target selection. The combined feature-set performed significantly better than all of the other feature-sets, surpassing the best task-specific featureset, $\left\{r_{1}, r_{2}\right\}$, by almost $7 \%$, and the best generalizable feature-set, $\overline{\Delta d o t}, \overline{\Delta D}$, by about $6 \%$.

As opposed to the task-specific features analysis, the results revealed that combining target radii with target distances yielded better accuracies than using each feature separately, but only if the distance was measured after the trial start, within the chosen time window. 


\subsection{Conclusion}

This chapter evaluated the usage of Fitts' $I D$ and its separable components, $D$ and $W$, as task specific features, as well as $\overline{\Delta d o t}$ and $\overline{\Delta D}$, as generalizable features to predict intended targets in undirected moving-target selection tasks. All of the evaluated feature-sets performed better than chance, and a zero-rule predictor, and the combination of both types of features yielded the best accuracy $(\sim 78 \%)$.

The generalizable features were calculated within a time-window ending at about twothirds of the selection time, heuristically selected based on participant gaze. Future work could explore automating this process by finding the optimal start and end window limits, by measuring different inputs. Furthermore, these times could be related to existing models, such as the Hick-Hyman Law [Hick 1952; Hyman 1953], presented in Equation (2.1). Notice, however, that in interactive contexts, the time window may not be necessary, since users may select their start and end times directly.

These results should be generalizable to different moving-target selection tasks, provided that additional factors, such as a greater number of spheres with different starting positions and different velocities, are taken into consideration. The potential of using other measures of difficulty (either objective or subjective) formulated specifically for moving-target selection to predict user intention should also be explored. 


\section{CHAPTER 4}

\section{Prospective Difficulty OF 2-D StATIC-tARGet AND MOVING-tARGet SELECTION}

Chapter 3 evaluated the hypothesis presented in Chapter 1 that $I D$ was predictive of user intention in undirected moving target selection, based on the premise that users form their intentions by minimizing their prospective effort, as described by ID. Due to the inadequacy of existing ID measures formulated specifically for moving-target selection, Chapter 3 resorted to using a static-target formulation of $I D$, specifically the Shannon model (Equation (2.5)) and its separate $D$ and $W$ components. Consistently with the results of Jagacinski et al. [1980] for the MT of moving-targets, the results revealed that such ID was limited in usefulness for predicting intention in moving-targets, thus, hinting at its inadequacy as a $P D$ measure for moving-target selection as well.

In order to address these limitations, this Chapter attempts to extend the $P D-I D$ paradigm to moving-target selection tasks. The proposed model is initially formulated and evaluated in 2-D tasks to avoid issues inherent to 3-D interaction, such as incorrect depth-perception, but bearing its extensibility to 3-D in mind. A general 2-D moving-target pointing task is presented in Figure 4.1, along with its relevant attributes.

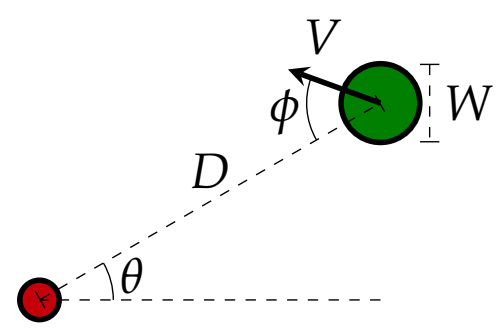

Figure 4.1. Measurements relevant to the target-selection tasks considered in this chapter. The red and the green circles indicate the starting positions of the cursor and the target, respectively. $D$ is the initial distance between the cursor and the target, $W$ is target width, $\theta$ is the initial angle between the target and the cursor, $V$ is the target speed, and $\phi$ is the target's movement direction relative to the initial cursor-target vector. 
To achieve this goal, the current chapter introduces three models based on Fitts' ID for the $P D$ of pointing at 2-D static targets, and 1-D and 2-D moving targets. The model formulae are presented below, in Sections 4.1.1-4.1.3.

\subsection{Formulations}

\subsubsection{PD formulation for 2-D static-target pointing tasks}

Two-dimensional static-target pointing tasks refer to tasks with initial target angle $\theta \in[0,360)^{\circ}$. For this type of task, the model proposed in this chapter separates the target-angle $(\theta)$ effect from the $I D=\log _{2}(2 D / W)$ term, such that

$$
P D=a_{\theta}+b \log _{2}\left(\frac{2 D}{W}\right)
$$

where $a_{\theta}$ and $b$ are empirically derived coefficients; $a_{\theta}$ is the per-angle $\theta$ intercept. This formulation is based on Fitts original formulation for $M T,{ }^{1}$ and the 2-D ID extensions by Murata and Iwase [2001], Appert et al. [2008], and Grossman and Balakrishnan [2004]. This equation is developed in Section 4.2.2.

\subsubsection{PD formulation for 1-D moving-target pointing tasks}

One-dimensional moving-target pointing tasks refer to tasks with initial target angle $\theta \in$ $\{0,180\}^{\circ}$, and target moving-direction $\phi \in\{0,180\}^{\circ}$. For this type of task, the proposed model is based on the two-part model by Hoffmann and Chan [2012] with per-speed $(V)$ regression coefficients, such that

$$
P D=a_{V}+b_{V} \sqrt{D}+c_{V} \log _{2}\left(\frac{2 D}{W}\right),
$$

where $a_{V}, b_{V}$, and $c_{V}$ are empirically derived coefficients. Based on an analogy between control-display gain $(G)$ and $V$, this formulation is inspired by the work of Shoemaker et al. [2012], who found that two-part models can be used to describe static-target pointing with different levels of $G$.

This formula is developed in Section 4.2.3. Additionally, as shown in Section 4.4.2.2, coefficients $a_{V}, b_{V}$, and $c_{V}$ appear to be linearly related to the speed $V$ of the target.

\subsubsection{PD formulation for 2-D moving-target pointing tasks}

Two-dimensional moving-target pointing tasks refer to tasks where the target's moving direction $\phi \in[0,360)^{\circ}$, regardless of the initial target angle $\theta$. For this type of task, the

\footnotetext{
${ }^{1}$ The choice of Fitts formulation for ID is explained in Section 4.2.1.
} 
proposed model is an extension of the 1-D moving-target model, separating the initial target distance $D$ into two components, such that

$$
\begin{gathered}
P D=a_{V}+b \sqrt{D_{s}}+c_{V} \sqrt{D_{m}}+d \log _{2}\left(\frac{2 D_{m}}{W}\right) \\
D_{s}=|D \sin \phi| \\
D_{m}=|D \cos \phi|,
\end{gathered}
$$

where $a_{V}, b, c_{V}$, and $d$ are empirically derived coefficients; $a_{V}$ and $c_{V}$ are per-speed $V$ coefficients. The development of this formula is both analytical, as explored in Section 4.2.4, and empirical, as explored in Section 4.4.2.2. Additionally, as shown in Section 4.4.2.2, $a_{V}$ and $c_{V}$ appear to be linearly related to the speed $V$ of the target.

\subsection{Modeling}

Based on the literature review presented in Chapter 2, and motivated by the goal of extending the $P D-I D$ paradigm to 2-D moving-target pointing tasks, this section presents an incremental development of such a model. First, the choice of the ID formula for the PD of 1-D static-target tasks is presented, followed by the extension to 2-D static-target tasks, 1-D moving-target tasks, and, finally, 2-D moving-target tasks.

\subsubsection{Choice of ID formulation}

In contrast with the majority of the post-1990's Fitts-related literature in HCI, Fitts' ID formulation is chosen over the Shannon formulation. The most important reason for this choice is the simplicity in deriving the formulae proposed to model prospective difficulty, notably the 2-D model presented in Section 4.2.2. Another important reason for this choice is the compatibility of Fitts' ID with prospective pointing tasks: if there were a prospective task in which the pointer started at the boundary of a static target (i.e., $D=W / 2$ ), the expected difficulty for an imagined movement should be 0 , allowing one to hypothesize the intercept $a=0$ in Equation (4.1) The latter is not the case for the Shannon $I D$, since the intercept represents a situation in which the pointer starts at the center of the target. Tasks in which $D<W / 2$, however, are out of the scope of this study as they may be hard to interpret prospectively, besides not being adequately modeled in the Fitts paradigm [Soukoreff and MacKenzie 2004, p. 768]. Lastly, choosing the Fitts $I D$ is consistent with the subjective difficulty literature, allowing comparison of the current results to previous work in the area.

Therefore, following the previous work in subjective difficulty and Fitts' Law [Delignières and Famose 1992; Slifkin and Grilli 2006; Grilli 2011; Chan and Hoffmann 2013], the model for the $P D$ of pointing at a $1-D$ static target is 


$$
\begin{aligned}
& P D=a+b I D \\
& I D=\log _{2}\left(\frac{2 D}{W}\right) .
\end{aligned}
$$

\subsubsection{Formulations of prospective difficulty in 2-D static-target pointing tasks}

To the author's knowledge, there is no extension to Fitts' original ID that explicitly accounts for the target angle $\theta$. Indeed, the 2-D extensions to Fitts' ID presented in Equations (2.10)-(2.12) are based on the Shannon formulation. However, analogous models based on Fitts original ID can be formulated as well. ${ }^{2}$ For example, Equation (2.10), by Murata and Iwase [2001], is analogous to

$$
I D_{M I}=\log _{2}\left(\frac{2 D}{W}\right)+c \sin \theta,
$$

Equation (2.11), by Appert et al. [2008], is analogous to

$$
I D_{A C B}=\log _{2}\left(\frac{2 D}{W}+\frac{2 D}{H}+0.6 \cos (\theta) \frac{2 D}{\min (W, H)}\right),
$$

and Equation (2.12), by Grossman and Balakrishnan [2004], is analogous to

$$
I D_{G B}=\log _{2}\left(\sqrt{f_{W}(\theta)\left(\frac{2 D}{W}\right)^{2}+f_{H}(\theta)\left(\frac{2 D}{H}\right)^{2}}\right) .
$$

These three formulations are easier to manipulate than their Shannon counterparts. For example, by isolating ID from the angle and shape effects in Equations (4.3) and (4.4), the three equations can be expressed in a similar form,

$$
I D_{2-\mathrm{D}}=\log _{2}\left(\frac{2 D}{W}\right)+f\left(\theta, \frac{W}{H}\right) .
$$

For Equation (4.2) there is no shape effect, only an angle effect expressed as

$$
f\left(\theta, \frac{W}{H}\right)=f(\theta)=c \sin \theta
$$

For Equation (4.3), the angle and shape effects are expressed as

$$
f\left(\theta, \frac{W}{H}\right)=\log _{2}\left(\frac{W}{H}+0.6 \cos (\theta) \frac{W}{\min (W, H)}+1\right) .
$$

\footnotetext{
${ }^{2}$ This is done by multiplying $D$ by 2 , and removing the " +1 " terms from the Shannon-derived formulae. A similar approach was taken by Shoemaker et al. [2012] to reformulate the distant pointing model by Kopper et al. [2010].
} 
Finally, the angle and shape effects for Equation (4.4) are expressed as

$$
f\left(\theta, \frac{W}{H}\right)=\log _{2}\left(\sqrt{f_{W}(\theta)+f_{H}(\theta)\left(\frac{W}{H}\right)^{2}}\right) .
$$

With these reformulations, Fitts' ID becomes nested within $I D_{2-\mathrm{D}}$ (i.e., $I D_{2-\mathrm{D}}=I D$, given $f(\theta, W / H)=0$ ). Based on this equivalency, the linear relation between $P D$ and $I D$ found in the literature also extends to $I D_{2-\mathrm{D}}$, thus

$$
P D=a+b I D_{2-\mathrm{D}}
$$

which can also be formulated as a model with one intercept per $(\theta, W / H)$ condition, such that

$$
P D=a_{\theta \times W / H}+b I D,
$$

where $a_{\theta \times W / H}=a+b f(\theta, W / H)$. For univariate targets, where $W=H$, the formula is further simplified to

$$
P D=a_{\theta}+b I D,
$$

where $a_{\theta}=a+b f(\theta)$. If no specific form of $f(\theta, W / H)$, or $f(\theta)$ is assumed, Equations (4.10) and (4.11) can be solved using the ubiquitous linear least-squares method, which does not suffer from the shortcomings of its non-linear counterpart described at the end of Section 2.1.2.2. For example, by using indicators, or dummy variables $\left(x_{i}\right)$ for the $i$ levels of $\theta$, Equation (4.11) can be represented as

$$
P D=\alpha_{1}+\alpha_{2} x_{2}+\alpha_{3} x_{3}+\ldots+\alpha_{i-1} x_{i-1}+\alpha_{i} x_{i}+b I D,
$$

such that

$$
\left(x_{2}, x_{3}, \ldots, x_{i-1}, x_{i}\right)=\left\{\begin{array}{lll}
(0,0, \ldots, 0,0) & \text { if } \theta=\theta_{1} \\
(1,0, \ldots, 0,0) & \text { if } \theta=\theta_{2} \\
(0,1, \ldots, 0,0) & \text { if } \theta=\theta_{3} \\
\ldots & & \\
(0,0, \ldots, 1,0) & \text { if } \theta=\theta_{i-1} \\
(0,0, \ldots, 0,1) & \text { if } \theta=\theta_{i},
\end{array}\right.
$$

where $\theta_{1}, \ldots, \theta_{i}$, are the different levels of $\theta$. 


\subsubsection{Formulations of prospective difficulty in 1-D moving-target pointing tasks}

As discussed in the Related Work section, the existing formulations for predicting $M T$ in moving-target selection are not compatible with the current $P D-I D$ framework. Equation (2.15), by Jagacinski et al. [1980], is simple but cannot be reduced to Fitts' Law, so its use would imply modeling static and moving targets differently; Equations (2.17) and (2.18), by Hoffmann [1991], are derived from Fitts' Law, but require the percentage of actual captures (unknown in prospective action), otherwise resulting in potentially harder and unreliable calculations. Therefore, it seems necessary to introduce another formulation to model the $P D$ of movingtarget selection, that is both simple and compatible with Fitts' ID.

To this end, an analogy between static-target selection under the effect of control-display gain, and moving target selection without gain is given. Under control-display gain,

$$
G=\frac{V_{\text {display }}}{V_{\text {control }}}
$$

the movement time (MT) required to reach for a static target located at position $x$ is

$$
\begin{aligned}
M T & =\frac{x}{V_{\text {display }}} \\
& =\frac{x}{G \times V_{\text {control }}} .
\end{aligned}
$$

On the other hand, with $G=1$ (i.e., $V_{\text {control }}=V_{\text {display }}$ ) the $M T$ required to reach for a target located at position $x$, and moving with velocity $V_{\text {target }}$ is

$$
M T=\frac{x}{V_{\text {control }}-V_{\text {target }}} .
$$

Combining (4.15) and (4.16) gives the equivalency

$$
G=1-\frac{V_{\text {target }}}{V_{\text {control }}}
$$

Thus, reaching for a static target with $G>0$ is equivalent to reaching for a moving target with $\left|V_{\text {control }}\right|>\left|V_{\text {target }}\right|$. Reaching for a static target with $G=0$ (i.e., when the display does not react to the control input) is equivalent to reaching for a moving target with $V_{\text {control }}=V_{\text {target }}-$ in both cases the target always remains at the same distance and pointing at it is impossible. Likewise, reaching for a static target with $G<0$ (i.e., when the display's velocity is opposite to the control input) is equivalent to reaching for a moving target with $\left|V_{\text {control }}\right|<\left|V_{\text {target }}\right|$-in both cases the target gets farther from the cursor as time elapses. The major shortcoming of this analogy is that it assumes that the user's control velocity is constant.

This is the basis for hypothesizing that two-part formulations model 1-D moving-target selection, analogous to the way Shoemaker et al. [2012] apply a two-part model to I-D static-

target selection with gain. Furthermore, based on the approximate equivalency between the 
two-part formulations of Welford and Hoffmann, described in Section 2.1.2.1, the latter should also model moving-target selection with the added benefit of mathematical correctness and, more importantly, the apparent linear relation between the regression coefficients and $V .^{3}$

For the prospective difficulty of I-D moving-target selection tasks, the proposed relationship is

$$
P D=a_{V}+b_{V} \sqrt{D}+c_{V} \log _{2}\left(\frac{2 D}{W}\right),
$$

where $V$ is the target velocity. The form of this model is similar to that of Equation (2.15), by Jagacinski et al. [1980], with $a_{V}=a-d(V+1)$, and $c_{V}=c(V+1)$. Aside from the $\log$ and square-root terms, the biggest difference in both models is that Jagacinski's model does not allow the effect of $D$ to be different for each $V$. However, it seems logical that higher target speeds will require faster reaction times, hence more ballistic-type motions, which are represented by the $\sqrt{D}$ term in Hoffmann's two-part model.

\subsubsection{Formulations of prospective difficulty in 2-D moving-target pointing tasks}

The motion required to point at a target moving in 2-D can be divided in two components, one perpendicular to the target's velocity, modeled by Equation (4.11), and one parallel to the target's velocity, modeled by Equation (4.18). Under these assumptions, the corresponding model is hypothesized as

$$
P D=a_{V \times \phi}+b_{V} \log _{2}\left(\frac{2 D_{s}}{W}\right)+c_{V} \sqrt{D_{m}}+d_{V} \log _{2}\left(\frac{2 D_{m}}{W}\right),
$$

where $D_{s}$ is the perpendicular, or "static distance," and $D_{m}$ is the parallel, or "moving distance." Since the true $D_{s}$ and $D_{m}$ are unknown in prospective movements, it is assumed that $D_{s}, D_{m}$, and $D$ form a right triangle as shown in Figure 4.2, so that $D_{s}=|D \sin \phi|$, and $D_{m}=|D \cos \phi|$, where $\phi$ is the target's movement direction relative to the initial cursor-target vector. ${ }^{4}$

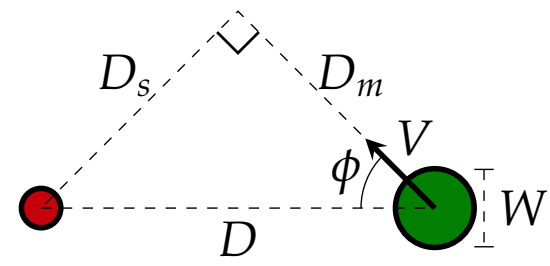

Figure 4.2. Two-dimensional moving-target model. The red circle is the starting point, the dark green circle is the moving target.

\footnotetext{
${ }^{3}$ It can be shown that the "Welford" coefficients in [Shoemaker et al. 2012, Table XI], with the corresponding transformations described in Equations (2.8)-(2.1.2.1), vary linearly with $G$.

${ }^{4}$ In the more general case, when $\theta$ and $\phi$ are known, $D_{s}=D \frac{\sin \phi}{\sin (\phi+\theta)}$ and $D_{m}=D \frac{\sin \theta}{\sin (\phi+\theta)}$.
} 
This simplification is compensated by having different $b_{V}, c_{V}$, and $d_{V}$ parameters per speed, and a different intercept $a_{V \times \phi}$ per velocity $(V, \phi)$, allowing $D_{s}$ and $D_{m}$ to be rescaled, which approximates the more general case in which $D_{s}, D_{m}$, and $D$ do not form a right triangle. The per-velocity intercept also allows for asymmetry on the effect of $\phi$.

Dropping the denominator within the $\log _{2}\left(2 D_{s} / W\right)$ term, and using the approximate log-square-root equivalence suggested by Hoffmann and Chan [2012] results in,

$$
P D=a_{V \times \phi}+b_{V} \sqrt{D_{s}}+c_{V} \sqrt{D_{m}}+d_{V} \log _{2}\left(\frac{2 D_{m}}{W}\right) .
$$

Under this model the complete motion would be described in three parts-two ballistic motions, one to align the cursor with the target's movement axis and one in-line with the target's movement axis, and a homing in motion in-line with the target's movement axis.

\subsection{Methods}

\subsubsection{Apparatus}

To evaluate the proposed models, an experiment was implemented using Scalable Vector Graphics (svG) [W3C 2011] and deployed in an anonymous, online Qualtrics survey.

\subsubsection{Participants}

Participants were recruited by distributing the survey link using social media, and yielded 49 respondents who answered the entire survey, out of 83 partial attempts-only the responses from the full attempts were retained. Twenty-one participants reported being students, and it was determined via timestamps that 25 participants were in the Americas, one in India, and the rest in either Europe or Africa.

Participants were aged 20 to 48 years old $(M=29.65, M d n=29)$; there were 14 females and 35 males. Most respondents reported being right handed (45), no respondents reported being ambidextrous.

\subsubsection{Procedure}

Upon opening the survey website, each participant was asked to accept a study consent form. If agreed, an animation of a moving object was presented. After playing the animation, the participant was asked if a moving object was visible on the screen-this question served as a browser check. After successfully completing the browser check, the participant was asked to fill in a short questionnaire concerning background and demographic information.

Subsequently, the participant was presented with two blocks of questions. In each question, the participant was asked to assess the prospective difficulty of touching a circular target from a starting point as fast as possible, without actually touching the screen or making any finger 
movements. The participant was advised to maximize the survey window prior to the start of each block, to avoid distractions and reduce scrolling. In the first block each target was static; in the second block each target was moving.

Each question was presented on a separate page and rated using radio buttons, as suggested by Van Schaik and Ling [2007]. In each question the participant judged pointing difficulty using a 9-point "Likert-type" scale, where 1 was labeled as "Very easy" and 9 was labeled as "Very difficult."

To give a scoring reference, a sample image (the anchor) corresponding to the middle difficulty (5), was given both before each block and in the header of each question. The same static-target anchor was given for both static and moving targets.

To prevent the participant from trying to execute the pointing task, the mouse pointer disappeared when hovering over each image. There was no time limit for each answer and, in the case of moving targets, the animations could be replayed at the participant's discretion. A screenshot of one of the moving-target questions is presented in Figure 4.3.

Given the following image, consider that the difficulty of touching the blue target starting from the red point is 5 .

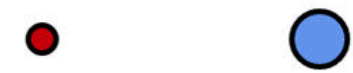

What is the difficulty of touching the green target as fast as possible starting from the red point?

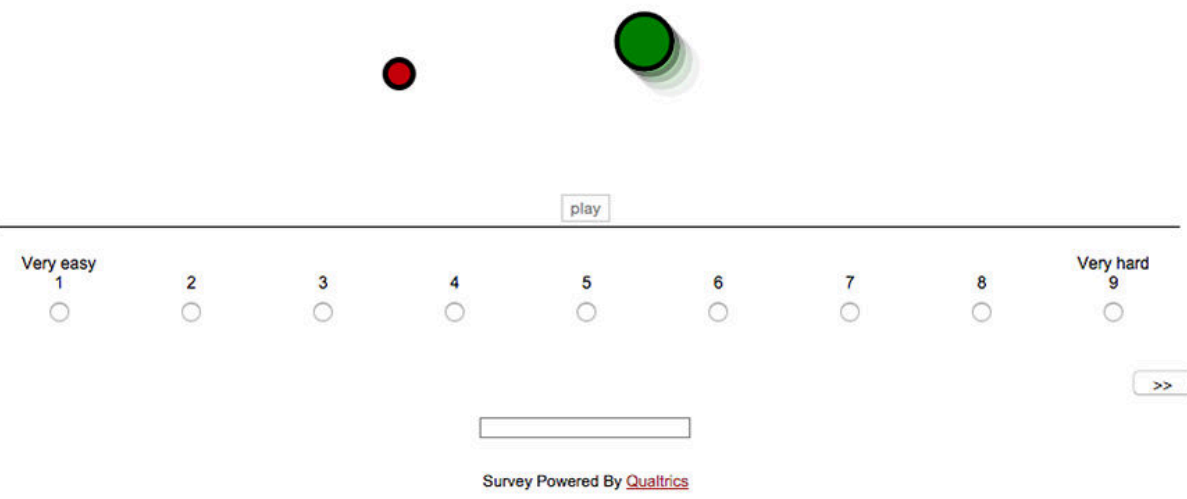

Figure 4.3. Screenshot of a moving-target question with $\phi=45^{\circ}$. The anchor is represented with a blue target.

To speed up survey completion, once the participant selected a $P D$ rating, the survey would automatically advance to the next question, as opposed to selecting an answer and clicking on the "next" button; the "next" button served only to skip questions, and there was 
no "back" button to revisit previous answers. The trade-off was that the participant could not correct misclicked responses, but this was mitigated by implementing "auto-advance" in the consent and browser-check questions, so that the participant would expect the same behavior when answering horizontally-arranged multiple-choice questions. Nevertheless, two participants commented on a few misclicks they made during the PD ratings.

At the end of each block, the participant was asked to rate how seriously they answered the preceding block, using a 1-5 "Likert-type" scale, where 1 was labeled as "Not seriously at all" and 5 was labeled as "Very seriously."

\subsubsection{Design}

A within-subjects, factorial design was used, with two blocks of trials.

The first block consisted of static targets. Factors were target distance, $D \in\{200,400\}$ pixels (px); target radius, $r \in\{20,40,80\} \mathrm{px}$; and target angle, $\theta \in\{-45,0,45\}^{\circ}$. Notice that $\theta$ was measured clockwise, consistently with the $y$-down screen coordinate system. Each of the 18 conditions was presented once to the participant in a random order. A sample of this task, with a depiction of its parameters, is shown in Figure 4.4.

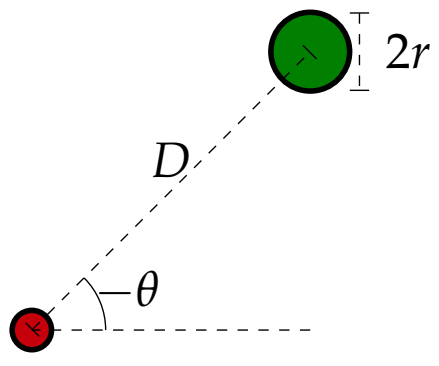

Figure 4.4. Static-target task. The red circle is the starting point, the green circle is the target.

The second block consisted of moving targets. Factors were initial target distance, $D \in\{200,400\} \mathrm{px}$; target radius, $r \in\{20,40,80\} \mathrm{px}$; target speed, $V \in\{200,400,800\} \mathrm{px} / \mathrm{s}$; and initial target-movement-direction, $\phi \in\{-45,0,45\}^{\circ}$; the initial target angle $\theta$ was always $0^{\circ}$. Each of the 54 conditions was presented once to the participant in a random order. A sample of this task, with a depiction of its parameters, is shown in Figure 4.5.

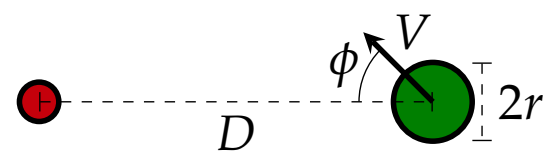

Figure 4.5. Moving-target task. The red circle is the starting point, the green circle is the moving target. 
The anchor for both static- and moving-target tasks was set as $(D=200 \mathrm{px}, r=20 \mathrm{px}$, $\theta=0^{\circ}, V=0 \mathrm{px} / \mathrm{s}$ ). The choice of the same static-target anchor for both types of task was based on the notion that moving-target selection is an extension to static-target selection.

\subsubsection{Data integrity}

Within the 49 complete survey attempts, two respondents rated more than $75 \%$ of their static-target responses with the minimum value (1), which suggests that they did not relate to the given anchor to rate their answers, therefore, all of their responses were discarded. Out of the 47 remaining attempts, six respondents rated more than $75 \%$ of their $1-\mathrm{D}$ moving-target responses with the minimum value (1), which suggests that they may have been executing a "crossing" task, in which they waited for the moving target to go over the cursor rather than touching the target as fast as possible, as instructed, ${ }^{5}$ therefore, all of their responses were removed. Within the 41 retained attempts, there were 2 missing answers in the static block, and none in the moving block. In total, there were 736 complete answers for the static block, and 2214 for the moving block.

\subsubsection{Statistical methods}

In general, the analyses in this chapter are conducted using Bayesian methods using Markov Chain Monte Carlo (MCMC). Bayesian methods have several advantages compared to traditional frequentist methods, including the use of prior knowledge, the generation of full parameter distributions, the ability to test complex models, and robustness against unbalanced samples and multiple comparisons [Kruschke et al. 2012].

By generating full distributions for the parameters of each model, Bayesian methods allow the formulation of probabilistic statements on the parameters and the models, as opposed to relying on approximate confidence intervals, or the seemingly unreliable $p$-values [Kruschke et al. 2012; Kaptein and Robertson 2012; Dragicevic et al. 2014]. Within this framework, testing for the "statistical significance" of null, or point hypothesis are avoided; instead, the emphasis is placed on the estimation of the different parameters, and the assessment of their credible values using the $95 \%$ highest density intervals (HDI) of their distributions. Following the recommendations from Gelman et al. [2013], the point estimates of each parameter are given using the median value of the posterior distribution.

\subsubsection{Models for inferential statistics}

Since participant responses are recorded in an ordinal scale, to evaluate the main effects of the experimental conditions and their interactions, on $P D$, the ordered probit regression model with per-subject intercept of the form

\footnotetext{
${ }^{5}$ This behavior was identified in the comments section of the survey, wherein a respondent reported being unsure if touching the target meant "crossing" it or "landing" in it, ultimately opting for the former.
} 


$$
\begin{aligned}
& P D_{i}=\left\{\begin{array}{lll}
1 & \text { if } \quad P D_{i}^{*}<\gamma_{1} \\
2 & \text { if } \quad \gamma_{1}<P D_{i}^{*} \leq \gamma_{2} \\
\cdots & & \\
8 & \text { if } & \gamma_{7}<P D_{i}^{*} \leq \gamma_{8} \\
9 & \text { if } & \gamma_{8}<P D_{i}^{*}
\end{array}\right. \\
& P D_{i}^{*}=\alpha_{i}+\mathbf{X}_{i} \boldsymbol{\beta}+\epsilon_{i} \\
& \alpha_{i} \sim \mathcal{N}\left(0, \sigma_{u}^{2}\right) \\
& \epsilon_{i} \sim \mathcal{N}(0,1)
\end{aligned}
$$

is used, where $\alpha_{i}$ is the per-subject intercept, and $\beta$ is the coefficient vector for $\mathbf{X}_{i}$ that includes different predictors according to the experimental block.

$P D^{*}$ and $\gamma_{k}$ are both defined in the probit scale, however, for simplicity, all the estimates in the inferential statistics sections are presented in the original 1-9 measurement scale. ${ }^{6}$ Once again, the emphasis is on estimating the effect sizes, corresponding to the coefficients of the different predictors within $\beta$, rather than on testing the statistical significance of those predictors.

The probit models are fit using мсмс sampling, using Stan [2014] via R [2014]. For each model, four MCMC chains of 5,000 iterations each, including 2,500 warm-up iterations, are drawn, for a total of 10,000 saved simulations. The convergence of the chains, and the goodness-of-fit of the model is verified to ensure the validity of the inference, but these metrics are not presented in the main body of the chapter.

\subsubsection{Models for Regression Analysis}

Unless otherwise indicated, a modified version of the Bayesian linear regression scripts by Kruschke et al. [2012] are used to regress the proposed models for PD. Each model is fit in Stan [2014], with four MсмС chains of 30,000 iterations, including 5,000 warm-up iterations, for a total of 100,000 saved iterations.

Model fit is assessed using the posterior distribution of the model's standard deviation $\sigma$, and $R^{2}$, calculated as the percentage of explained variance [Gelman and Hill 2007, p. 41],

$$
R^{2}=1-\frac{\sigma^{2}}{s_{y}^{2}},
$$

where $s_{y}$ is the standard deviation of $y$. The central value of this distribution is close to the so-called adjusted r-squared $\left(R_{a d j}^{2}\right) .{ }^{7}$ As opposed to the traditional $R^{2}$, used in most of the

\footnotetext{
${ }^{6}$ The scaling procedure consists in dividing each coefficient in $\beta$ by $M\left(\gamma_{2}-\gamma_{1}, \ldots, \gamma_{8}-\gamma_{7}\right)$.

${ }^{7}$ The equivalency between the adjusted and unadjusted $R^{2}$ is given by, $R_{a d j}^{2}=1-\left(1-R^{2}\right) \frac{n-1}{n-p-1}$, where $n$ is the number of data points, and $p$ is the number of fitted parameters, not including the intercept.
} 
Fitts' literature, this estimate does not necessarily increase with the addition of new predictors, which facilitates direct model comparison between nested models of different complexities.

The point estimates of $R^{2}$ may also be lower than those obtained using least-squares regression, since the $R^{2}$ value is optimized in the latter, but not in Bayesian linear regression. The difference in point estimates, however, is likely small, and less interesting than the $95 \%$ credible intervals of the $R^{2}$ distribution.

\subsubsection{Hypotheses}

All of the following hypotheses assume univariate targets in a 2-D space, i.e., circles. Thus, the difference between task dimensions (1-D, and 2-D) is given by the angles $\theta$ and $\phi$ of the target, with respect to the starting position.

\subsubsection{Static-target selection}

H1. Fitts' ID model with per-angle intercept, described in Equation (4.11), models the prospective difficulty of two-dimensional static-target selection tasks better than the classic Fitts' ID model with single intercept, described in Equation (4.1).

\subsubsection{Moving-target selection}

H2. Hoffmann's two-part model with per-velocity coefficients, described in Equation (4.18), accurately models the prospective difficulty of one-dimensional moving-target selection tasks.

H2.1. The coefficients $a_{V}, b_{V}$, and $c_{V}$ of Equation (4.18) vary linearly with $V$.

H3. The three-part model with per-speed coefficients, and per-velocity intercept, described in Equation (4.20), accurately models the prospective difficulty of two-dimensional moving-target selection tasks.

H3.1. In Equation (4.20) the coefficients $b_{V}, c_{V}$, and $d_{V}$ vary linearly with $V$, as well as the coefficients $a_{V \times \phi}$ within the same angle $\phi$.

\subsection{Analysis}

\subsubsection{Static-target block}

\subsubsection{Inferential statistics}

To evaluate the effects on $P D$ of $D, r, \theta$, and their interactions, the ordered probit regression of Equation (4.21) is fit with the following 13 predictors:

- An indicator for $D=400$ 
- Two indicators for $r \in\{40,80\}$

- Two indicators for $\theta \in\{-45,45\}$

- Two indicators for $D \times r$

- Two indicators for $D \times \theta$

- Four indicators for $r \times \theta$,

which implies that the base values, i.e., $\mathbf{X}_{i}=\mathbf{0}$, are set to $D=200, r=20$, and $\theta=0$.

Main Effects. The estimates in Figure 4.6 show that there are strong main effects of $D$, and $r$, but relatively small effects of $\theta$.

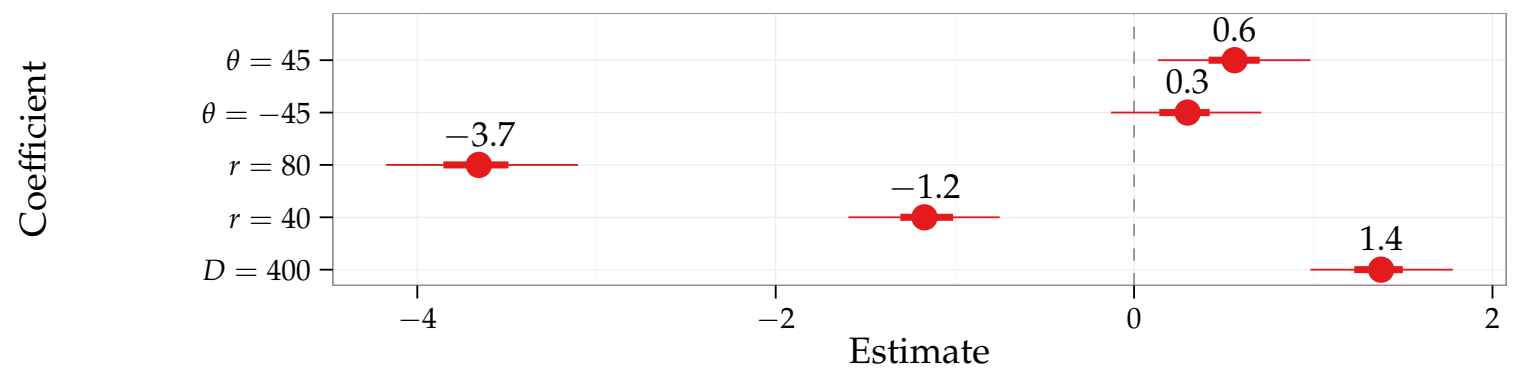

Figure 4.6. Main-effect coefficient plot for the fitted ordered probit model on static-target $P D$ ratings. Points, thick lines, and thin lines represent the medians, 50\%, and 95\% HDI for each of the coefficients in $\beta$.

As expected, $P D$ increases with $D$, and decreases with larger radii $r$. Similarly, downward targets $(\theta=45)$ were perceived as more difficult than horizontal ones, by about $0.56,95 \%$ HDI $[0.13,0.98]$. There is also some evidence that the angle effect on $P D$ is asymmetrical, with downward angles being perceived as more difficult than upward angles, $p\left(\beta_{\theta=45}>\beta_{\theta=-45}\right)=0.89$; however, the average difference in $P D$ between upward and downward angles is very small, $\operatorname{Mdn}\left(\beta_{\theta=45}-\beta_{\theta=-45}\right)=0.26,95 \%$ HDI $[-0.15,0.69]$. This is surprising, and contradicts the findings of Whisenand and Emurian [1995], who found that upward angles took more time to be reached; this discrepancy could indicate that $P D$ is not completely consistent with $M T$.

Two-way Interactions. As shown in Figure 4.7, the interaction effects have small magnitudes compared to the main effects, indicating that most of the changes in $P D$ are explained by the latter.

The $D \times r$ interaction shows that, at $D=400$, the main, negative effect of $r=80$, $\operatorname{Mdn}\left(\beta_{r=80}\right)=-3.66,95 \%$ HDI $[-4.17,-3.1]$, is slightly attenuated by about $0.62,95 \%$ HDI $[0.15,1.07]$. 


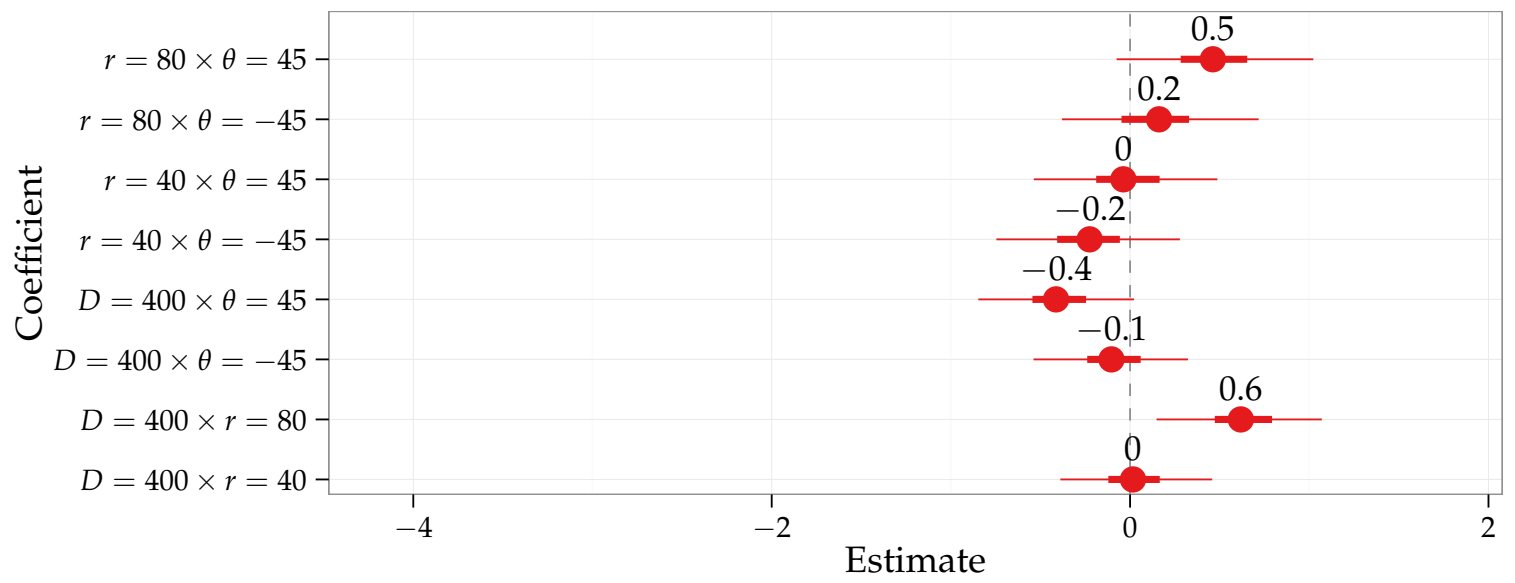

Figure 4.7. Two-way interaction coefficient plot for the fitted ordered probit model on static-target $P D$ ratings. Points, thick lines, and thin lines represent the medians, $50 \%$, and 95\% HDI for each of the coefficients in $\beta$.

Similarly, the $D \times \theta$ interaction shows that, at $D=400$, both main angle effects are considerably reduced and made almost symmetrical,

$$
\begin{aligned}
\operatorname{Mdn}\left(\beta_{\boldsymbol{\theta}=-45}+\beta_{D=400 \times \boldsymbol{\theta}=-45}\right) & =0.19,95 \% \text { HDI }[-0.22,0.61], \text { vs. } \\
\operatorname{Mdn}\left(\beta_{\boldsymbol{\theta}=45}+\beta_{D=400 \times \boldsymbol{\theta}=45}\right) & =0.15,95 \% \text { HDI }[-0.27,0.56] ;
\end{aligned}
$$

suggesting that at high distances, angle differences of $\theta= \pm 45$ contribute less to $P D$.

On the contrary, on targets with radii $r>20$ the asymmetrical effect of $\theta$ observed in the main effects is exacerbated,

$$
\begin{aligned}
& \operatorname{Mdn}\left[\left(\beta_{\theta=45}+\beta_{r=40 \times \theta=45}\right)-\left(\beta_{\theta=-45}+\beta_{r=40 \times \theta=-45}\right)\right]=0.45,95 \% \text { HDI }[0.03,0.9], \text { and } \\
& \operatorname{Mdn}\left[\left(\beta_{\theta=45}+\beta_{r=80 \times \theta=45}\right)-\left(\beta_{\theta=-45}+\beta_{r=80 \times \theta=-45}\right)\right]=0.56,95 \% \text { HDI }[0.11,1.04] .
\end{aligned}
$$

\subsubsection{Regression analysis}

Prospective difficulty ratings are grouped by $(D, r, \theta)$ condition, and summarized using means.

First, following Equation (4.1), a simple linear regression of the form

$$
\overline{P D} \sim \mathcal{N}\left(a+b I D, \sigma^{2}\right)
$$

is fit. The results, presented on Table 4.1, show that the fit is quite good in terms of $R^{2}$. However, Figure 4.8 shows that the regression line consistently underestimates downward 
targets $\left(\theta=45^{\circ}\right.$, represented by the green dots), and overestimates some of the horizontal targets angles $\left(\theta=0^{\circ}\right.$, represented by the red dots).

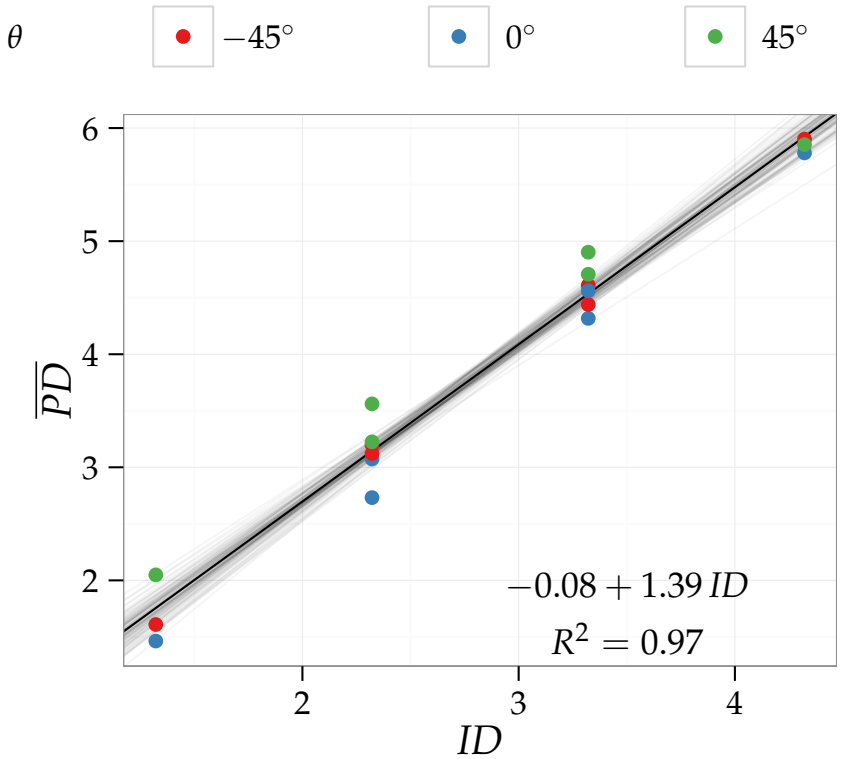

Figure 4.8. Regression for $\overline{P D}=a+b I D$. The thick black line represents the posterior median for the intercept and slope, the gray lines represent 100 posterior simulations from intercept and slope.

Table 4.1. Posterior medians (first row) and 95\% HDI (second row) of the of the regression estimates for $\overline{P D}=a+b I D$.

\begin{tabular}{cccc}
$a$ & $b$ & $\sigma$ & $R^{2}$ \\
\hline-0.08 & 1.39 & 0.24 & 0.97 \\
{$[-0.44,0.29]$} & {$[1.27,1.51]$} & {$[0.16,0.34]$} & {$[0.94,0.99]$}
\end{tabular}

These results suggest that a model accounting for target direction might model the $P D$ of pointing at 2-D static-targets more accurately. Thus, following Equation (4.11), a linear regression with per-angle intercept of the form

$$
\overline{P D} \sim \mathcal{N}\left(a_{\theta}+b I D, \sigma^{2}\right)
$$

is fit.

The regression, displayed in Figure 4.9, shows a great fit, with a very good $R^{2}=0.99$, and no visible non-random patterns around the regression lines. The results, presented in Table 4.2, show estimates of $\sigma$ and $R^{2}$ that are better (lower for $\sigma$, and higher for $R^{2}$ ), and 
more precise than those of the simple linear model, shown in Table 4.1. This is reflected in Figure 4.9, which shows that all of the points in the dataset fall within the lines formed by the posterior draws of $a_{\theta}$ and $b$, with no visible non-random patterns.

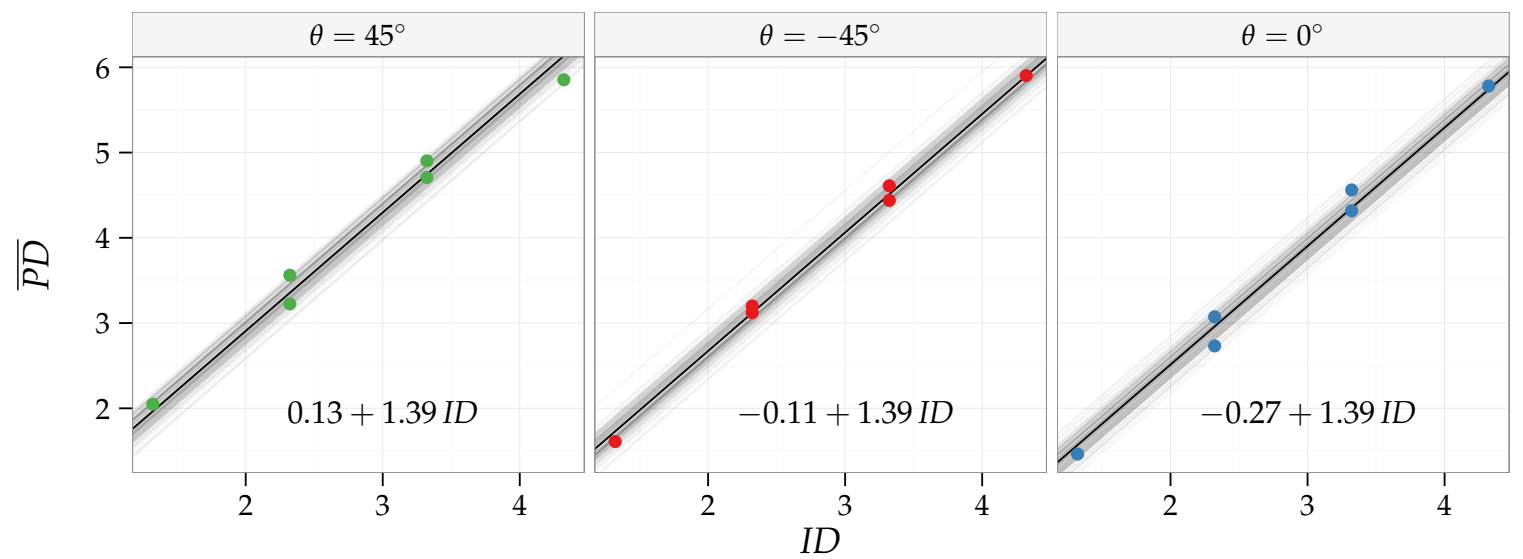

Figure 4.9. Regression for $\overline{P D}=a_{\theta}+b I D$. The thick black lines represent the posterior median for each intercept and slope, the gray lines represent 100 posterior simulations from intercept and slope.

Table 4.2. Posterior medians (first row) and 95\% HDI (second row) of the of the regression estimates for $\overline{P D}=a_{\theta}+b I D$.

\begin{tabular}{cccccc}
$a_{\theta=0}$ & $a_{\theta=-45}$ & $a_{\theta=45}$ & $b$ & $\sigma$ & $R^{2}$ \\
\hline-0.27 & -0.11 & 0.13 & 1.39 & 0.16 & 0.99 \\
{$[-0.55,0.02]$} & {$[-0.4,0.17]$} & {$[-0.16,0.4]$} & {$[1.3,1.48]$} & {$[0.11,0.24]$} & {$[0.97,0.99]$}
\end{tabular}

\subsubsection{Participant performance and self assessment}

The median time participants spent in each question was $5 \mathrm{~s}, 95 \%$ HDI $[1,20]$, and only in five trials the survey window went out of focus. The median seriousness rating with which participants assessed their performance was 4, 95\% HDI [3,5], with 1 being "Not seriously at all," and 5 "Very seriously."

\subsubsection{Moving-target block}

\subsubsection{Inferential statistics}

To evaluate the effects on $P D$ of $D, r, V$, and $\phi$, and their interactions, the ordered probit regression of Equation (4.21) is fit with the following 25 predictors:

- One indicator for $D=400$ 
- Two indicators for $r \in\{40,80\}$

- Two indicators for $V \in\{-400,-800\}$

- Two indicators for $\phi \in\{-45,45\}$

- Two indicators for $D \times r$

- Two indicators for $D \times V$

- Two indicators for $D \times \phi$

- Four indicators for $r \times V$

- Four indicators for $r \times \phi$

- Four indicators for $V \times \phi$,

implying that the base values, i.e., $\mathbf{X}_{i}=0$, are set to $D=200, r=20, V=-200$, and $\phi=0$.

Main Effects. The estimates in Figure 4.10 show that there are strong main effects of $r, V$, and $\phi$. As in the static block, $P D$ decreased with larger $r$, however, as opposed to the static block, $P D$ was virtually unaffected by $D, M d n=-0.02,95 \%$ HDI $[-0.35,0.33]$. Additionally, $P D$ increased with $|V|$, and almost symmetrically with $\phi= \pm 45, M d n\left(\beta_{\phi=45}-\beta_{\phi=-45}\right)=0.07$, $95 \%$ HDI $[-0.32,0.44]$. Given the $G-V$ analogy presented in Section 4.2.3, this is consistent with the subjective assessments of Shoemaker et al. [2012], who found that higher G levels were perceived as more difficult.

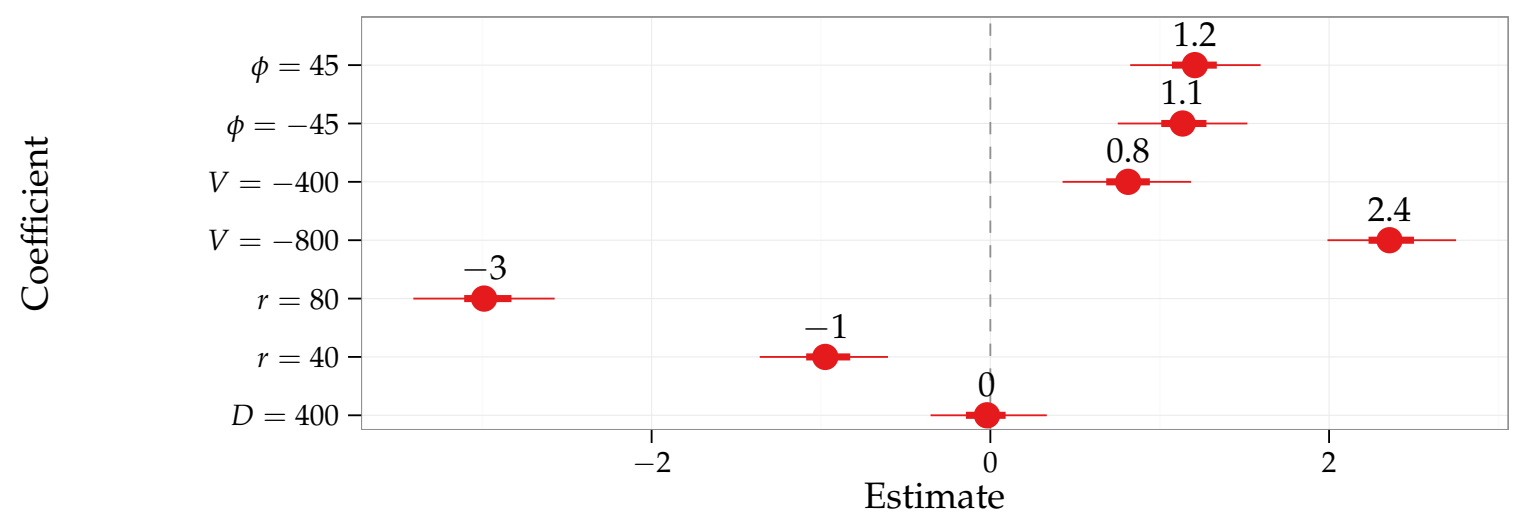

Figure 4.10. Main-effect coefficient plot for the fitted ordered probit model on moving-target $P D$ ratings. Points, thick lines, and thin lines represent the medians, 50\%, and 95\% HDI for each of the coefficients in $\beta$. 
Two-way Interactions. Figure 4.11 shows that interaction effects have small magnitudes compared to the main effects, indicating that most of the changes in $P D$ are explained by the latter.

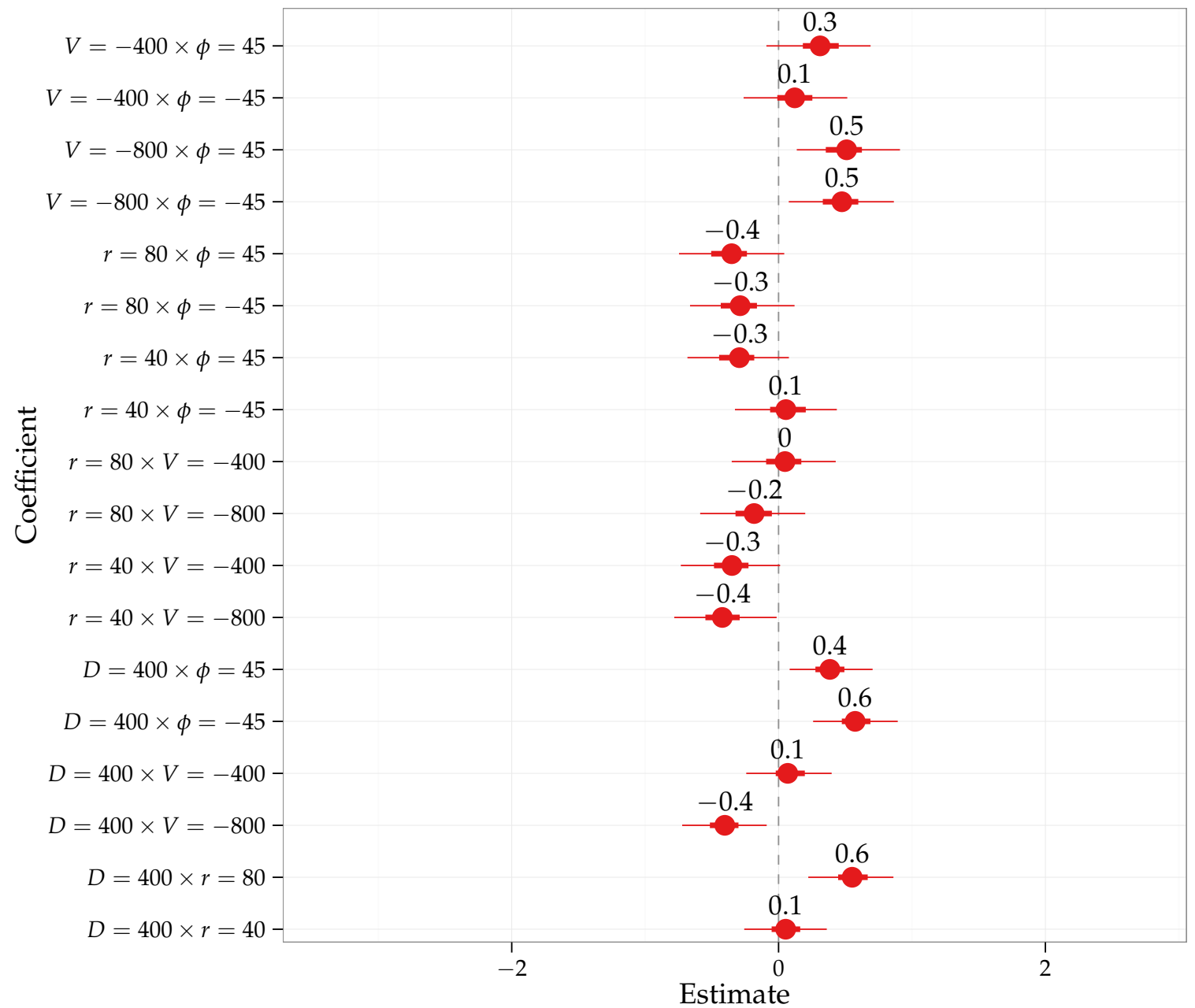

Figure 4.11. Two-way interaction coefficient plot for the fitted ordered probit model on movingtarget $P D$ ratings. Points, thick lines, and thin lines represent the medians, 50\%, and $95 \%$ HDI for each of the coefficients in $\beta$.

Even though the main effect of $D$ was very weak, the interactions between $D$ and the other main effects show some interesting, non-additive relations. For levels of $r<80, D$ had a close-to-zero effect on $P D$; however, when $r=80, D$ added about $0.55,95 \%$ HDI $[0.22,0.86]$, to the prospective difficulty of the task, which indicates that the effect of $r$ is lower on larger distances (similar to the static block). Likewise, for levels of $|V|<800, D$ had a close-to-zero effect on $P D$; however, when $V=-800, D$ actually made the task easier on average by 0.55 , 
$95 \%$ HDI $[0.22,0.86]$, indicating perhaps that $D$ gives more reaction time to participants on high speed scenarios, and this is perceived as easier. Also, the $D \times \phi$ interaction had a positive effect on $P D$, which indicates that non-zero angles are perceived as more difficult with higher distances.

Concerning the interactions between $r$ and the other main effects, $r>20$ mostly decrease the effects of $V$ and $\phi, M d n\left(\beta_{r>20 \times(V \neq-200, \phi \neq 0)}\right)=-0.24,95 \%$ HDI $[-0.78,-0.01]$. The interaction effects of $r=80 \times V=-800$, and $r=40 \times \phi=-45$, however, are close to 0 , $\operatorname{Mdn}\left(\beta_{r=80 \times V=-400}, \beta_{r=40 \times \phi=-400}\right)=0.05,95 \%$ HDI $[-0.35,0.43]$.

Finally, concerning the $V \times \phi$ interaction, when $|V|>200$, the angle effects are somehow exacerbated. At $V=-400$, the $\phi= \pm 45$ effects increase by about $0.22,95 \%$ HDI $[-0.26,0.52]$, and at $V=-800$, the $\phi= \pm 45$ effects increase by about $0.49,95 \%$ HDI $[0.08,0.86]$.

Symmetry of target movement-direction effects. In general, the main and interaction effects of $\phi$ seem to be symmetrical, which suggests that the three-part model with per $V \times \phi$ intercept, described in Equation (4.20), is unnecessarily complex, and a simpler, nested model with intercepts varying only by $V$, such that

$$
P D=a_{V}+b_{V} \sqrt{D_{s}}+c_{V} \sqrt{D_{m}}+d_{V} I D_{m},
$$

may be sufficient. Indeed, this model already includes the interactions between $D, r, V$, and $\phi$ in its non-constant terms.

\subsubsection{Regression analysis}

Prospective difficulty ratings are grouped by $(D, r, V, \phi)$ condition, and summarized using means.

One-dimensional moving-target model. A subset of the moving-target tasks, where $\phi=0$, is fit using Hoffmann's two-part model with per-velocity coefficients, described in Equation (4.18). Therefore, a linear regression of the form

$$
\overline{P D} \sim \mathcal{N}\left(a_{V}+b_{V} \sqrt{D}+c_{V} I D, \sigma^{2}\right)
$$

is fit. The resulting estimates, presented in Table 4.3, show that the fit is very good in terms of $R^{2}$, with credible values above 0.92 , and distributions of $a, b$, and $c$ that increase in magnitude with $V$. To interpret the results graphically, Formula (4.26) is reparametrized to yield one simple regression line per $(D, V)$ condition, each with intercept $\alpha_{D, V}=a_{V}+b_{V} \sqrt{D}$, and slope $c_{V}$.

The six regression lines, presented in Figure 4.12, show that the model fits the data very well, without any noticeable non-random patterns around the regression lines. Based on the intercepts of the reparametrized regression lines, the graph suggests that the $P D-I D$ baseline increases with $|V|$, and decreases with $D$. Based on the slopes, the graph suggests that the rate of $P D$ change per $I D$ change increases slightly with $|V|$. 


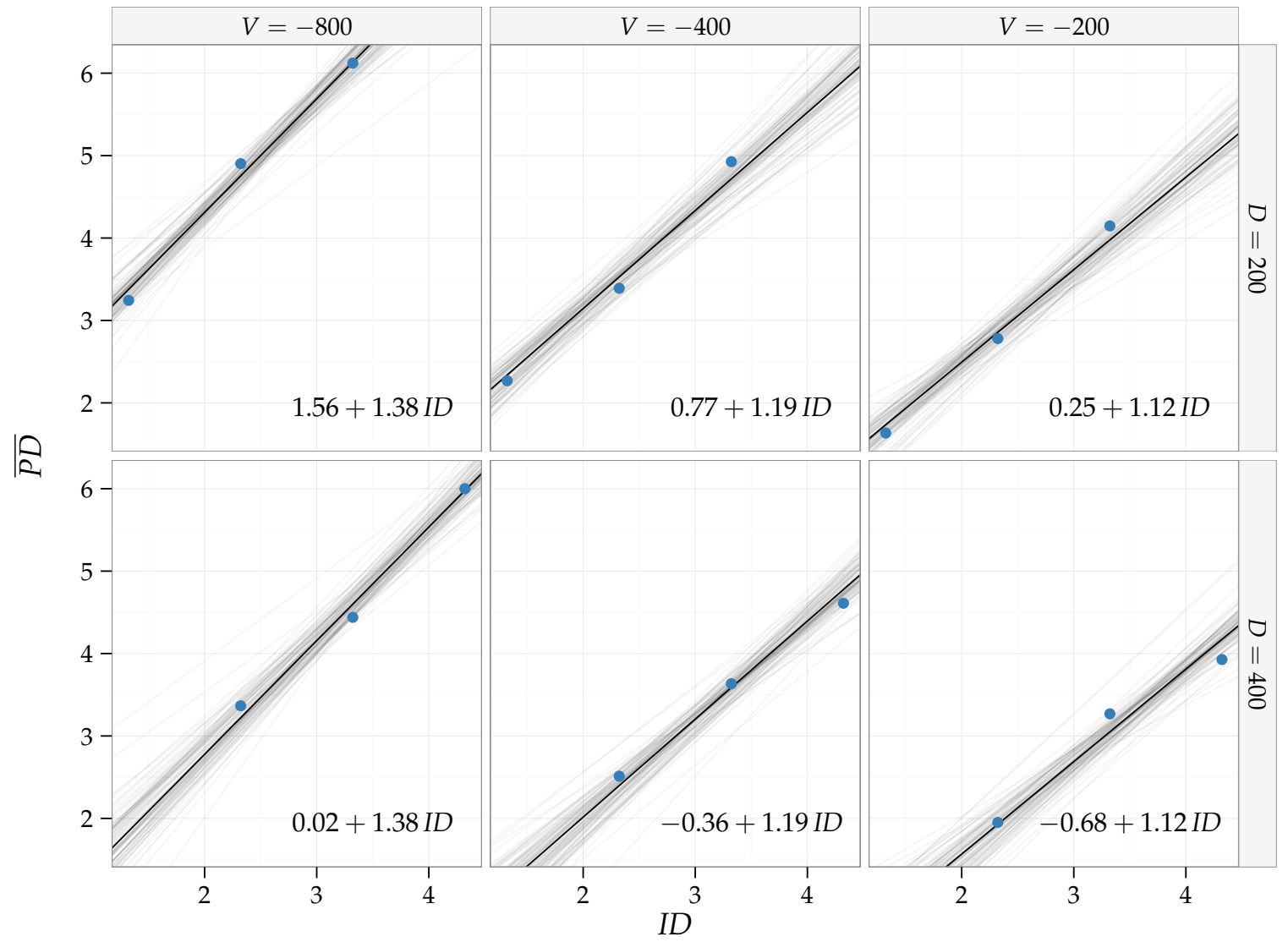

Figure 4.12. Regression for $\overline{P D}=a_{V}+b_{V} \sqrt{D}+c_{V} I D$ reparametrized to yield six simple regression lines per $(D, V)$ condition, each with intercept $\alpha_{D, V}=a_{V}+b_{V} \sqrt{D}$, and slope $c_{V}$. The thick black lines represent the posterior median for each intercept and slope, the gray lines represent 100 posterior simulations from intercept and slope. 
Table 4.3. Regression estimates for $\overline{P D}=a_{V}+b_{V} \sqrt{D}+c_{V} I D$.

\begin{tabular}{ccc} 
Estimand & $M d n$ & $95 \%$ HDI \\
\hline$a_{V=-200}$ & 2.49 & {$[1.32,3.64]$} \\
$a_{V=-400}$ & 3.5 & {$[2.33,4.63]$} \\
$a_{V=-800}$ & 5.26 & {$[4.1,6.42]$} \\
$b_{V=-200}$ & -0.16 & {$[-0.24,-0.08]$} \\
$b_{V=-400}$ & -0.19 & {$[-0.27,-0.11]$} \\
$b_{V=-800}$ & -0.26 & {$[-0.34,-0.18]$} \\
$c_{V=-200}$ & 1.12 & {$[0.88,1.36]$} \\
$c_{V=-400}$ & 1.19 & {$[0.95,1.43]$} \\
$c_{V=-800}$ & 1.38 & {$[1.14,1.62]$} \\
$\sigma$ & 0.22 & {$[0.13,0.36]$} \\
$R^{2}$ & 0.97 & {$[0.92,0.99]$}
\end{tabular}

Change of coefficients per $V$. Consistently with the $k-G$ analyses from Shoemaker et al. [2012], linear regressions of the point estimates from Table 4.3- $\hat{a}_{V}=M d n\left(a_{V}\right), \hat{b}_{V}=\operatorname{Mdn}\left(b_{V}\right)$, and $\hat{c}_{V}=M d n\left(c_{V}\right)$-on target velocity $V$ are calculated using the default least-squares method in $\mathrm{R}$ [2014]. The results, given in Table 4.4, show that there is some evidence of the linear relation between $V$ and $\hat{a}, V$ and $\hat{b}$, as well as $V$ and $\hat{c}$.

Table 4.4. Least-squares estimates for the regressions of the point estimates from Table $4.3-\hat{a}_{V}=\operatorname{Mdn}\left(a_{V}\right), \hat{b}_{V}=\operatorname{Mdn}\left(b_{V}\right)$, and $\hat{c}_{V}=\operatorname{Mdn}\left(c_{V}\right)$-on target velocity $V$.

\begin{tabular}{ccccc} 
Model & Intercept & Slope & $\hat{\sigma}$ & $\hat{R}^{2}$ \\
\hline$\hat{a}_{V} \sim \mathcal{N}\left(\alpha_{1}+\alpha_{2} V \times 10^{-3}, \sigma_{a}^{2}\right)$ & 1.61 & -4.58 & 0.07 & 1 \\
$\hat{b}_{V} \sim \mathcal{N}\left(\beta_{1}+\beta_{2} V \times 10^{-3}, \sigma_{b}^{2}\right)$ & -0.12 & 0.17 & 0 & 1 \\
$\hat{c}_{V} \sim \mathcal{N}\left(\zeta_{1}+\zeta_{2} V \times 10^{-3}, \sigma_{c}^{2}\right)$ & 1.03 & -0.43 & 0.01 & 0.99
\end{tabular}

Similar to the analyses of Shoemaker et al. [2012], however, the least-squares standard deviation estimates, $\hat{\sigma}_{a}, \hat{\sigma}_{b}$, and $\hat{\sigma}_{c}$, are too optimistic, as they ignore the uncertainty on $a, b$, and $c$. This prevents the calculation of accurate confidence/credible intervals for $\alpha_{1}, \alpha_{2}, \beta_{1}, \beta_{2}$, $\zeta_{1}$, and $\zeta_{2}$.

To give a better sense of uncertainty, a hierarchical model, which simultaneously estimates the parameters in Tables 4.3 and 4.4, is fit. Following the recommendations from Gelman [2006] for standard deviation parameters on hierarchical models, especially those with a low number of groups (in the current study, for example, $\#(V)=3$ ), half-Cauchy priors ${ }^{8}$ are chosen for $\sigma_{a}, \sigma_{b}, \sigma_{c}$, and $\sigma$, with scale parameter $A=5$, which is wide with respect to the range of the data (1-9). Since the center of the $\alpha_{1}, \alpha_{2}, \beta_{1}, \beta_{2}, \zeta_{1}$, and $\zeta_{2}$ distributions are

\footnotetext{
${ }^{8}$ The half-Cauchy distribution is equivalent to the right half of a 1-degree-of-freedom $t$ distribution.
} 
not expected to have a considerable change with respect to the least-squares estimates from Table 4.4, the latter are used as mean parameters for their priors; still, a wide prior variance parameter $\left(10^{2}\right)$ on their distribution is used to allow for some flexibility both in the mean and the variance of the marginal posteriors. These weakly-informative priors give reasonable constraints to the posterior distributions, improving the MсмC sampler convergence, while minimally affecting the inferences from the data. In any case, the goal of this hierarchical model is not to get accurate point estimates, especially of $a, b, c, \alpha, \beta$, and $\zeta$, for which rough estimates are already known; rather, the goal is to get more realistic uncertainty around $\alpha, \beta$, and $\zeta$, and better estimates of $\sigma_{a}, \sigma_{b}$, and $\sigma_{c}$.

The model is fit using Stan [2014], with four MCMC chains of 5,000 iterations, including 2,500 warm-up iterations, for a total of 10,000 saved iterations. ${ }^{9}$ The levels and estimates of the model are shown in Table 4.5.

Table 4.5. Levels and estimates of the hierarchical regression $\overline{P D}_{i} \sim \mathcal{N}\left(a_{V[i]}+b_{V[i]} \sqrt{D_{i}}+c_{V[i]} I D_{i}, \sigma^{2}\right) . \hat{\alpha}_{1}, \hat{\alpha}_{2}, \hat{\beta}_{1}, \hat{\beta}_{2}, \hat{\zeta}_{1}$, and $\hat{\zeta}_{2}$ correspond to the least-squares estimates from Table 4.4.

\begin{tabular}{cccc} 
Model & Estimand & $M d n$ & $95 \%$ HDI \\
\hline$a_{V} \sim \mathcal{N}\left(\alpha_{1}+\alpha_{2} V \times 10^{-3}, \sigma_{a}^{2}\right)$ & $a_{V=-200}$ & 2.49 & {$[1.46,3.49]$} \\
& $a_{V=-400}$ & 3.47 & {$[2.55,4.38]$} \\
& $a_{V=-800}$ & 5.25 & {$[4.15,6.32]$} \\
$b_{V=-200}$ & -0.16 & {$[-0.23,-0.09]$} \\
$b_{V} \sim \mathcal{N}\left(\beta_{1}+\beta_{2} V \times 10^{-3}, \sigma_{b}^{2}\right)$ & $b_{V=-400}$ & -0.19 & {$[-0.26,-0.13]$} \\
& $b_{V=-800}$ & -0.26 & {$[-0.34,-0.19]$} \\
$\mathcal{c}_{V} \sim \mathcal{N}\left(\zeta_{1}+\zeta_{2} V \times 10^{-3}, \sigma_{c}^{2}\right)$ & $c_{V=-200}$ & 1.12 & {$[0.89,1.34]$} \\
& $c_{V=-400}$ & 1.2 & {$[0.98,1.39]$} \\
$\sigma \sim$ half-Cauchy $(5)$ & $c_{V=-800}$ & 1.38 & {$[1.15,1.6]$} \\
$\alpha_{j} \sim \mathcal{N}\left(\hat{\alpha}_{j}, 10^{2}\right)$ & $\sigma$ & 0.21 & {$[0.13,0.34]$} \\
$\sigma_{a} \sim$ half-Cauchy $(5)$ & $\alpha_{1}$ & 1.62 & {$[-2.42,6.31]$} \\
$\beta_{j} \sim \mathcal{N}\left(\hat{\beta}_{j}, 10^{2}\right)$ & $\alpha_{2}$ & -4.53 & {$[-11.88,3.67]$} \\
$\sigma_{b} \sim$ half-Cauchy $(5)$ & $\sigma_{a}$ & 0.86 & {$[0,4.72]$} \\
\hline$\zeta_{j} \sim \mathcal{N}\left(\hat{\zeta}_{j}, 10^{2}\right)$ & $\beta_{2}$ & -0.13 & {$[-1.11,0.74]$} \\
$\sigma_{c} \sim$ half-Cauchy $(5)$ & $\sigma_{b}$ & 0.16 & {$[-1.48,2.05]$} \\
\hline & $\zeta_{1}$ & 1.03 & {$[-2.45,3.75]$} \\
\hline & $\zeta_{2}$ & -0.41 & {$[-6.03,5.34]$} \\
& $\sigma_{c}$ & 0.42 & {$[0,3.69]$}
\end{tabular}

The hierarchical-model results show that the standard deviations $\sigma_{a}, \sigma_{b}$, and $\sigma_{c}$ are much higher than those from Table 4.4. Correspondingly, the intervals around $\alpha_{1}, \alpha_{2}, \beta_{1}, \beta_{2}$,

\footnotetext{
${ }^{9}$ Due to the time it took the MCMC sampler to run the iterations compared to the linear regression models, the number of iterations was reduced with respect to the latter. Nevertheless, the 10,000 iterations were sufficient for the sampler to converge.
} 
$\zeta_{1}$, and $\zeta_{2}$ are wider than those that would be obtained from the least-squares regression. Nevertheless, the similarity in the distributions of parameters $a, b, c$, and $\sigma$ between Tables 4.3 and 4.5 suggests that, in spite of the wide uncertainty, linear relations between $V$ and $a$, $V$ and $b$, as well as $V$ and $c$, are plausible and congruent with the data.

Two-dimensional moving-target model. As suggested in the inferential statistics section, the three-part model with per-speed intercepts described in Equation (4.25) is evaluated for all the moving-target tasks. Therefore, a linear regression of the form

$$
\overline{P D} \sim \mathcal{N}\left(a_{V}+b_{V} \sqrt{\overline{D_{s}}}+c_{V} \sqrt{\overline{D_{m}}}+d_{V} I D_{m}, \sigma^{2}\right)
$$

is fit. The resulting estimates are presented in Table 4.6.

Table 4.6. Regression estimates for $\overline{P D}=a_{V}+b_{V} \sqrt{D_{s}}+c_{V} \sqrt{D_{m}}+d_{V} I D_{m}$.

\begin{tabular}{ccc} 
Estimand & $M d n$ & $95 \% \mathrm{HDI}$ \\
\hline$a_{V=-200}$ & 2.98 & {$[2.23,3.7]$} \\
$a_{V=-400}$ & 3.59 & {$[2.86,4.33]$} \\
$a_{V=-800}$ & 5.96 & {$[5.25,6.72]$} \\
$b_{V=-200}$ & 0.08 & {$[0.06,0.1]$} \\
$b_{V=-400}$ & 0.1 & {$[0.09,0.12]$} \\
$b_{V=-800}$ & 0.1 & {$[0.09,0.12]$} \\
$c_{V=-200}$ & -0.22 & {$[-0.27,-0.17]$} \\
$c_{V=-400}$ & -0.24 & {$[-0.29,-0.18]$} \\
$c_{V=-800}$ & -0.31 & {$[-0.36,-0.26]$} \\
$d_{V=-200}$ & 1.31 & {$[1.16,1.47]$} \\
$d_{V=-400}$ & 1.41 & {$[1.26,1.56]$} \\
$d_{V=-800}$ & 1.41 & {$[1.26,1.56]$} \\
$\sigma$ & 0.26 & {$[0.21,0.32]$} \\
$R^{2}$ & 0.97 & {$[0.96,0.98]$}
\end{tabular}

Table 4.6 shows that all of the coefficients are "statistically significant" in the sense that their $95 \%$ HDI do not include 0 . However, the low values of $b_{V}$ indicate that $\sqrt{D_{s}}$ contributes very little to $P D$, and its coefficients show very small variations among levels of $V$, indicating that the contribution of the "static distance" $D_{s}$ to $P D$ is minimally affected by $V$. There is no credible difference between $b_{V=-400}$ and $b_{V=-800}$, and the difference between $b_{V=-400}$ and $b_{V=-200}$ is so small, that a target starting at $D=400$, moving with $(V=-800, \phi= \pm 45)$ would be perceived as harder than a target with the same starting position, but moving with $(V=-200, \phi= \pm 45)$, only by 0.37 units, $95 \%$ HDI $[-0.06,0.8]$.

It is possible that the differences between values of $b_{V}$, and $d_{V}$ will be larger for targets with different configurations, however, with the present experimental-design and observed data this is not the case. This suggests that a model with fixed $b$, and $d$, such that 


$$
P D=a_{V}+b \sqrt{D_{s}}+c_{V} \sqrt{D_{m}}+d I D_{m}
$$

may be sufficient to adequately fit the data. Therefore, its corresponding linear regression,

$$
\overline{P D} \sim \mathcal{N}\left(a_{V}+b \sqrt{D_{s}}+c_{V} \sqrt{D_{m}}+d I D_{m}, \sigma^{2}\right),
$$

is fit. The resulting estimates are presented in Table 4.7. The estimates are very similar to those in Table 4.6; the previous differences between $b_{V=-200}$ and $b_{V \in\{-400,-800\}}$, and between $d_{V=-200}$ and $d_{V \in\{-400,-800\}}$ are now represented in $a_{V}$, whose values present wider changes between levels of $V$ than in the previous model. In terms of goodness of fit both models are very similar, there is no credible $R^{2}$ difference, and the difference of 0.01 in $\sigma$, in both the median and the upper bound of the $95 \% \mathrm{HDI}$, is not of practical importance in the 1-9 measurement scale.

To interpret the results graphically, Formula (4.29) is reparametrized to yield one simple regression line per $(D, V,|\phi|)$ condition, each with intercept $\alpha_{D, V,|\phi|}=a_{V}+b \sqrt{D|\sin \phi|}+c_{V} \sqrt{D|\cos \phi|}$, and slope $d$. The 12 regression lines presented in Figure 4.13 show that the model fits the data very well, with no noticeable non-random patterns around the regression lines.

Table 4.7. Regression estimates for $\overline{P D}=a_{V}+b \sqrt{D_{s}}+c_{V} \sqrt{D_{m}}+d I D_{m}$.

\begin{tabular}{ccc} 
Estimand & $M d n$ & $95 \% \mathrm{HDI}$ \\
\hline$a_{V=-200}$ & 2.74 & {$[2.04,3.43]$} \\
$a_{V=-400}$ & 3.72 & {$[3.03,4.42]$} \\
$a_{V=-800}$ & 6.08 & {$[5.39,6.76]$} \\
$b$ & 0.1 & {$[0.09,0.11]$} \\
$c_{V=-200}$ & -0.22 & {$[-0.27,-0.17]$} \\
$c_{V=-400}$ & -0.23 & {$[-0.28,-0.19]$} \\
$c_{V}=-800$ & -0.3 & {$[-0.35,-0.26]$} \\
$d$ & 1.38 & {$[1.29,1.47]$} \\
$\sigma$ & 0.27 & {$[0.21,0.33]$} \\
$R^{2}$ & 0.97 & {$[0.96,0.98]$}
\end{tabular}

Based on the intercepts of the reparametrized regression lines, the graph suggests that the $P D-I D$ baseline increases with $\phi$ and $|V|$, and decreases with $D$. The slopes suggest that the rate of $P D$ change per $I D$ stays roughly similar for all conditions.

Change of coefficients per $V$. In a manner analogous to the analyses presented in the 1-D moving-target section, linear regressions on the per- $V$ parameter estimates from Table $4.7-$ $\hat{a}_{V}=\operatorname{Mdn}\left(a_{V}\right)$, and $\hat{c}_{V}=\operatorname{Mdn}\left(c_{V}\right)$-are first calculated using the default least-squares method in $\mathrm{R}$ [2014]. The results, shown in Table 4.8, give some evidence in support of the linear relation between $V$ and $\hat{a}$, as well as $V$ and $\hat{c}$. 


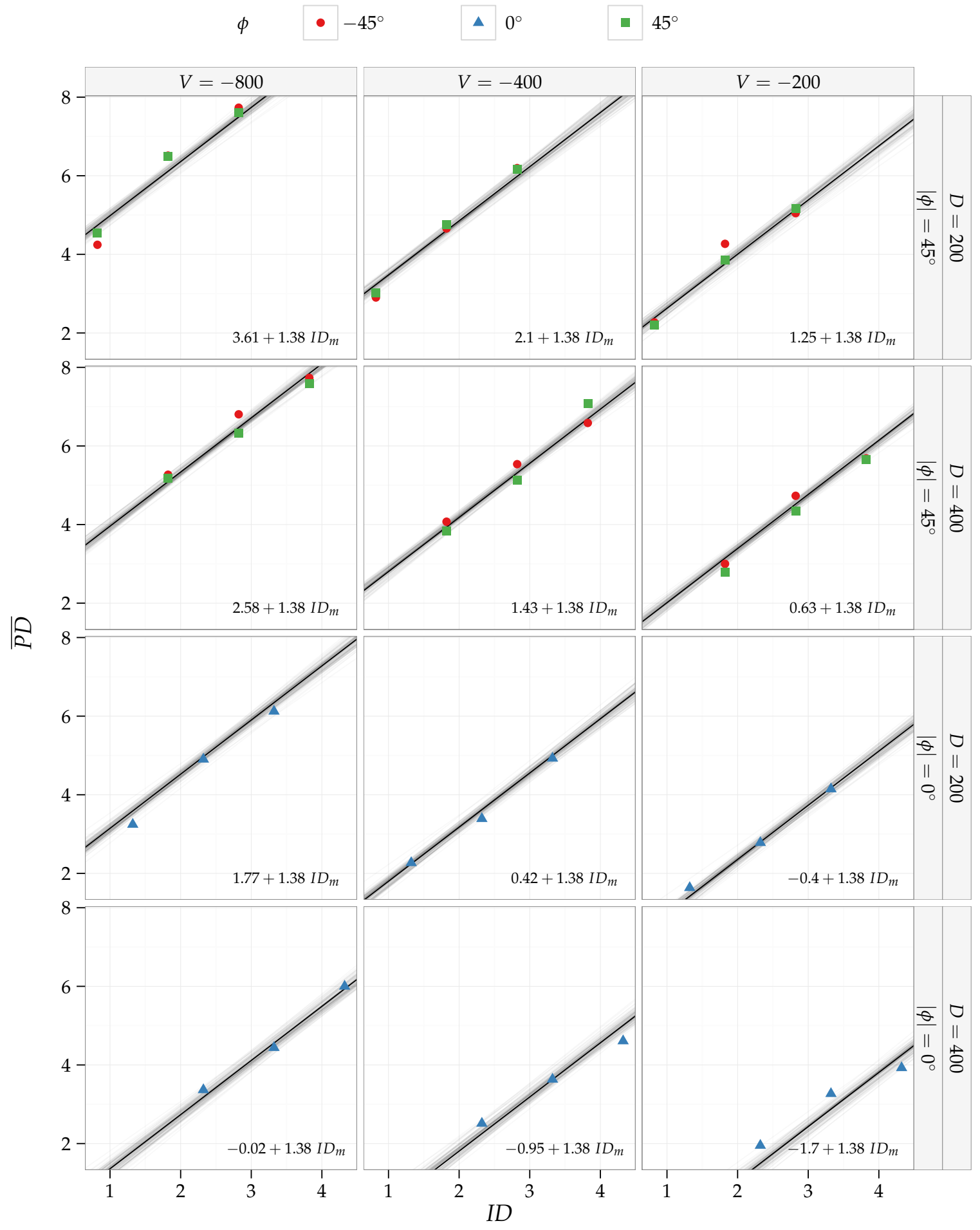

Figure 4.13. Regression for $\overline{P D}=a_{V}+b \sqrt{D_{s}}+c_{V} \sqrt{D_{m}}+d I D_{m}$ reparametrized to yield twelve simple regression lines per $(D, V,|\phi|)$ condition, each with intercept $\alpha_{D, V,|\phi|}=a_{V}+b \sqrt{D|\sin \phi|}+c_{V} \sqrt{D|\cos \phi|}$, and slope $d$. The thick black lines represent the posterior median for each intercept and slope, the gray lines represent 100 posterior simulations from intercept and slope. 
Table 4.8. Least-squares estimates for the regressions of the point estimates from Table $4.7-\hat{a}_{V}=\operatorname{Mdn}\left(a_{V}\right)$, and $\hat{c}_{V}=\operatorname{Mdn}\left(c_{V}\right)$ —on target speed $V$.

\begin{tabular}{ccccc} 
Model & Intercept & Slope & $\hat{\sigma}$ & $\hat{R}^{2}$ \\
\hline$\hat{a}_{V} \sim \mathcal{N}\left(\alpha_{1}+\alpha_{2} V \times 10^{-3}, \sigma_{a}^{2}\right)$ & 1.56 & -5.61 & 0.1 & 1 \\
$\hat{c}_{V} \sim \mathcal{N}\left(\zeta_{1}+\zeta_{2} V \times 10^{-3}, \sigma_{c}^{2}\right)$ & -0.19 & 0.14 & 0.01 & 0.92
\end{tabular}

Once again, the least-squares estimates $\hat{\sigma}_{a}$, and $\hat{\sigma}_{c}$ are too optimistic, as they ignore the uncertainty on $a$, and $c$. Thus, to have a better sense of uncertainty around the estimates, and assess the plausibility of a linear relation between $V$ and $a$, as well as $V$ and $c$, a hierarchical model which simultaneously estimates the parameters in Tables 4.7 and 4.8 , is fit. The hierarchical model reuses the least-squares estimates $\hat{\alpha}_{1}, \hat{\alpha}_{2}, \hat{\zeta}_{1}$, and $\hat{\zeta}_{2}$ from Table 4.8 as priors for the mean parameters of $\alpha_{1}, \alpha_{2}, \zeta_{1}$, and $\zeta_{2}$, respectively, as well as the distribution medians $M d n(b)$, and $M d n(d)$ from Table 4.7, as priors for the mean parameters of $b$, and $d$, respectively; the wide prior variance $\left(10^{2}\right)$ allows for some flexibility both in the mean and the variance of the marginal posteriors. Similar to the 1-D moving-target hierarchical model, half-Cauchy priors are used for the variance parameters $\sigma_{a}, \sigma_{c}$, and $\sigma$.

The model is fit using Stan [2014], with four MCMC chains of 5,000 iterations, including 2,500 warm-up iterations, for a total of 10,000 saved iterations. The levels and estimates of the model shown in Table 4.9, again, show higher estimates for $\sigma_{a}$, and $\sigma_{b}$ than those obtained in Table 4.8, and correspondingly, wider intervals around $\hat{\alpha}_{1}, \hat{\alpha}_{2}, \hat{\zeta}_{1}$, and $\hat{\zeta}_{2}$ than those that would be obtained from the least-squares regression. Nevertheless, the minimal change in the distributions of $a, b, c, d$, and $\sigma$ between Tables 4.7 and 4.8 suggests that, in spite of the wide uncertainty, the linear relations between $V$ and $a$, and $V$ and $c$ are plausible and congruent with the data.

\subsubsection{Participant performance and self assessment}

The median time participants spent in each question was $3 \mathrm{~s}, 95 \%$ HDI $[1,11]$, and only in 11 trials the survey window went out of focus. Participants replayed the animation in $15.31 \%$ of the trials, but within those trials the median number of replays was $1,95 \%$ HDI $[1,3]$. The median seriousness rating with which participants assessed their performance was 4, 95\% HDI [3,5], with 1 being "Not seriously at all," and 5 "Very seriously."

\subsection{Discussion}

Results are summarized according to the study hypotheses, each followed by a discussion. After the hypotheses discussions, the limitations of the current study and possible future work are presented. 
Table 4.9. Levels and estimates of the hierarchical regression $\overline{P D}_{i} \sim \mathcal{N}\left(a_{V[i]}+b \sqrt{D_{s i}}+c_{V[i]} \sqrt{D_{m i}}+d I D_{m i}, \sigma^{2}\right) . \quad \hat{\alpha}_{1}, \hat{\alpha}_{2}, \hat{\zeta}_{1}$, and $\hat{\zeta}_{2}$ correspond to the least-squares estimates from Table 4.8. $\hat{b}$, and $\hat{d}$ correspond to the distribution medians for $b$, and $d$ from Table 4.7 .

\begin{tabular}{cccc} 
Model & Estimand & Mdn & $95 \%$ HDI \\
\hline$a_{V} \sim \mathcal{N}\left(\alpha_{1}+\alpha_{2} V \times 10^{-3}, \sigma_{a}^{2}\right)$ & $a_{V=-200}$ & 2.7 & {$[2.06,3.33]$} \\
& $a_{V=-400}$ & 3.78 & {$[3.19,4.36]$} \\
$b \sim \mathcal{N}\left(\hat{b}, 10^{2}\right)$ & $a_{V=-800}$ & 6.06 & {$[5.4,6.74]$} \\
& $b$ & 0.1 & {$[0.09,0.11]$} \\
$c_{V} \sim \mathcal{N}\left(\zeta_{1}+\zeta_{2} V \times 10^{-3}, \sigma_{c}^{2}\right)$ & $c_{V=-200}$ & -0.22 & {$[-0.26,-0.18]$} \\
& $c_{V=-400}$ & -0.24 & {$[-0.28,-0.2]$} \\
$d \sim \mathcal{N}\left(\hat{d}, 10^{2}\right)$ & $c_{V=-800}$ & -0.3 & {$[-0.35,-0.26]$} \\
$\sigma \sim$ half-Cauchy $(5)$ & $d$ & 1.38 & {$[1.28,1.46]$} \\
$\alpha_{j} \sim \mathcal{N}\left(\hat{\alpha}_{j}, 10^{2}\right)$ & $\sigma$ & 0.26 & {$[0.22,0.33]$} \\
$\sigma_{a} \sim$ half-Cauchy $(5)$ & $\alpha_{1}$ & 1.58 & {$[-2.41,5.3]$} \\
$\zeta_{j} \sim \mathcal{N}\left(\hat{\zeta}_{j}, 10^{2}\right)$ & $\alpha_{2}$ & -5.61 & {$[-12.19,1.22]$} \\
$\sigma_{c} \sim$ half-Cauchy $(5)$ & $\sigma_{a}$ & 0.64 & {$[0,4.18]$} \\
\hline & $\zeta_{1}$ & -0.18 & {$[-0.7,0.43]$} \\
& $\zeta_{2}$ & 0.15 & {$[-0.94,1.28]$} \\
& $\sigma_{c}$ & 0.08 & {$[0,0.6]$}
\end{tabular}

\subsubsection{Prospective difficulty of static-target selection}

H1. Fitts' ID model with per-angle intercept, described in Equation (4.11), models the prospective difficulty of two-dimensional static-target selection tasks better than the classic Fitts' ID model with single intercept, described in Equation (4.1). Partially supported.

Both the fixed intercept model of Equation (4.1),

$$
P D=a+b I D,
$$

and the per-angle intercept model of Equation (4.11),

$$
P D=a_{\theta}+b I D
$$

yielded very good fits with credible values of $R^{2}=[0.94,0.99]$, and $R^{2}=[0.97,0.99]$, respectively. However, compared to the results of the fixed intercept model, presented in Table 4.1, the results of the per-angle intercept model presented in Table 4.2 showed slightly better, and more precise estimates of $\sigma$ and $R^{2}$. The difference in precision, $\operatorname{Mdn}\left(\sigma_{1}-\sigma_{2}\right)=0.07$, $95 \%$ HDI $[-0.04,0.2]$, however represents less than $1 \%$ of the total $1-9$ scale range. 
Even though the 2-D model is "significantly better" in statistical terms, ${ }^{10}$ the difference in precision between both models is not of practical significance. The importance of the model, however, lies in its flexibility and extensibility to other angles, therefore, $H 1$ is partially supported.

In any case, the fact that $I D$ was linearly related to $P D$, supports the usage of the chosen 9-point rating scale, with labels at the poles, and a middle anchor, to measure prospective difficulty. This point is crucial to validate the $P D$ ratings for moving-target selection using this scale.

\subsubsection{Prospective difficulty of moving-target selection}

H2. Hoffmann's two-part model with per-velocity coefficients, described in Equation (4.18), accurately models the prospective difficulty of one-dimensional moving-target selection tasks. Supported.

The 1-D moving-target selection model of Equation (4.18),

$$
P D=a_{V}+b_{V} \sqrt{D}+c_{V} I D,
$$

yielded a very good fit, with credible values of $R^{2}=[0.92,0.99]$, presented in Table 4.3, and regression lines that closely follow the data with no visible non-random patterns in the residuals, as shown in Figure 4.12. This was expected given the structural similarity between Equation (4.18) and Jagacinski's "general" model in Equation (2.15), as well as the G- $V_{\text {target }}$ analogy, presented in Section 4.2.3.

H2.1. The coefficients $a_{V}, b_{V}$, and $c_{V}$ of Equation (4.18) vary linearly with $V$. Supported.

The results suggest that coefficients $a_{V}, b_{V}$, and $c_{V}$ of Equation (4.18) vary linearly with $V$. The analogy with gain, for which these approximated coefficients also seem to increase linearly, also supports this hypothesis.Using the credible intervals presented in Table 4.5 allows PD to be predicted using different $V$, while still propagating the uncertainty in these estimates.

H3. The three-part model with per-speed coefficients, and per-velocity intercept, described in Equation (4.20), accurately models the prospective difficulty of two-dimensional moving-target selection tasks. Supported.

It was determined that the three-part model with per-speed coefficients, and per-velocity intercept of Equation (4.20),

$$
P D=a_{V \times \phi}+b_{V} \sqrt{D_{s}}+c_{V} \sqrt{D_{m}}+d_{V} I D_{m},
$$

\footnotetext{
${ }^{10}$ Which can also verified using a classical $F$-test, not shown.
} 
was unnecessarily complex for the evaluated dataset; however, the simpler model with per-speed intercept and coefficient $c$ described in Equation (4.28),

$$
P D=a_{V}+b \sqrt{D_{s}}+c_{V} \sqrt{D_{m}}+d I D_{m}
$$

yielded a very good fit, with credible values of $R^{2}=[0.96,0.98]$, presented in Table 4.7, and regression lines that closely follow the data, with no visible non-random patterns in the residuals, as shown in Figure 4.13.

Since Equation (4.28) is nested within Equation (4.20), this implies that the latter also fits the data well, therefore $H 3$ is supported. Even though the simpler model was sufficient for the purposes of this chapter, Equation (4.20) should not be discarded for future analyses, especially those having a wider variety of angles, in which angle asymmetry might have a larger effect.

H3.1. In Equation (4.20) the coefficients $b_{V}, c_{V}$, and $d_{V}$ vary linearly with $V$, as well as the coefficients $a_{V \times \phi}$ within the same angle $\phi$. Partially Supported.

The results suggest that coefficients $a_{V}$, and $c_{V}$ of Equation (4.28) increase linearly with $V$. Contrary to what was hypothesized, $b$, and $d$ were almost constant across $V$. For $b$, this suggests that the prospective ballistic motion parallel to the target movement is minimally affected by $V$. For $d$, this suggests that the effect of a same target width does not change by $V$, as shown in the interaction plot in Figure 4.11. Using the credible intervals presented in Table 4.9, this allows $P D$ to be predicted using different $V$, while still propagating the uncertainty in these estimates.

\subsection{Conclusion}

Contrary to most Fitts-related studies in HCI, which have focused mostly on modeling performance in static-target pointing tasks, the main goal of this study was to explore the $P D$ of moving-target pointing tasks in 2-D. This goal was achieved by developing and empirically evaluating models for 2-D static-target pointing, and 1-D and 2-D moving-target pointing. To the author's knowledge, this is the first study to evaluate pointing tasks in which the target is not moving directly toward, or directly away from the cursor, making the models closer to generalization than some of the work in the literature.

The proposed models have, in the author's opinion, relatively simple formulations. These models are inspired on previous work on the MT of 2-D static-target tasks [Murata and Iwase 2001; Appert et al. 2008; Grossman and Balakrishnan 2004], as well as two-part models [Welford et al. 1969; Hoffmann and Chan 2012], which have proven effective to model static-target selection with different levels of $G$ [Shoemaker et al. 2012]. Incidentally, the empirical data analysis was made using Bayesian methods, less prevalent in HCI yet very powerful-in this 
chapter, in particular, hierarchical Bayesian regressions allowed to propagate the uncertainty on the main model parameters to secondary regressions made on those parameters themselves.

As will be discussed in Chapter 6, there are many opportunities to extend the current work. Hopefully, this will incentivize and facilitate further HCI research on moving-target selection and subjective difficulty, which remain overshadowed by static-target selection and performance. Additionally, it is hoped that the analytical methods presented in this chapter will be considered as a viable alternative to the prevailing frequentist methods in HCI, and motivate more analyses to go beyond "statistical significance." 


\section{CHAPTER 5}

\section{Performance And intention in Directed 3-D moving-target SELECTION}

Chapters 1-3 hypothesized that ID was predictive of user intention in moving target selection, based on the premise that users form their intentions by minimizing their prospective effort, as described by $I D$. The results from Chapter 3, however, revealed that separating the $W$ and $D$ components was more effective for predicting intention, in a similar way as the models proposed in Chapter 4 separate $D$ from $I D$ to predict $P D$.

This similarity suggests that the proposed models may also be predictive of user intention, but this relation needs to be further explored. In particular, in order to predict user intention in VR, these models need to be extended to $3^{-D}$, and evaluated during action execution. To achieve these goals, this chapter explores the applicability of the PD models from Chapter 4 to predict selection performance. The performance predictions are, in turn, used to predict user intention by integrating them into the framework described in Chapter 3.

In order to generalize the results from Chapters 3 and 4, this chapter includes a wider span of experimental factors, including more levels of velocity, target angle, and number of spheres. Additionally, this chapter includes tasks in which users do not have to wait for the targets to be within their reach, and tasks in which users must choose a specific target among multiple others.

\subsection{Extending the 2-D formulae to 3-D}

One advantage of the 2-D model proposed in Chapter 4 is that it can theoretically be used to model 3-D target-selection tasks. Consider the general 3-D moving-target pointing task presented in Figure 5.1. Even though the target is moving in $3^{-D}$, the target velocity $(\mathbf{V})$ and initial target position (D) define a right triangle with sides $D, D_{m}$, and $D_{s}$, which, together with $W$, are the inputs in the model in Equation (4.20).

In addition to the target altitude $\theta$, present in 2-D tasks, 3-D tasks require an additional angle to define the target position, in this case a $z-x$ azimuth angle $\alpha$ is chosen. Due to depthperception problems in stereoscopic displays [Grossman and Balakrishnan 2004; Bowman et al. 


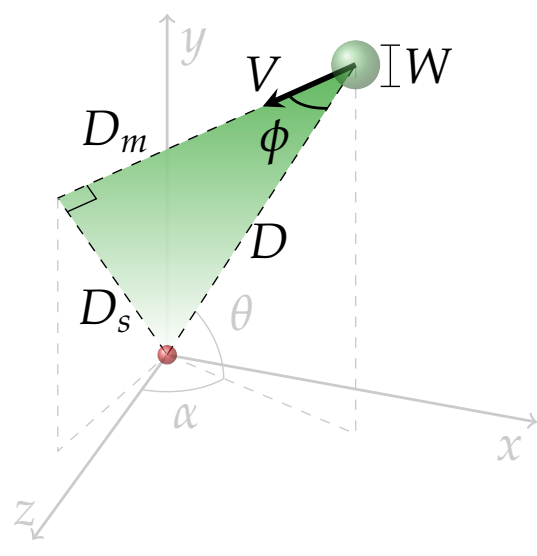

Figure 5.1. Three-dimensional moving-target model. The red and green spheres indicate the starting positions of the cursor and the target, respectively. The initial target point (D) is determined by the initial cursor-target distance $(D), z-x$ azimuth angle $(\alpha)$, and altitude angle $(\theta)$.

2004], targets with identical $(\mathbf{V}, D)$, but displayed at different screen depths, given by $(\theta, \alpha)$, may be perceived with different $P D$. This effect has previously been observed for the $M T$ required to acquire 3-D static targets [Teather and Stuerzlinger 2011].

Analogous to 2-D tasks, the initial angular coordinates $(\alpha, \theta)$ could be represented with different $a_{V \times \alpha \times \theta}$ intercepts. Yet again, it is hypothesized that, as long as the target is defined within the user's field of view, the initial $\alpha$ and $\theta$ will have a minimal effect, since these angles change as the target moves.

Likewise, the target orientation can no longer be solely defined with $\phi$. In fact, as shown in Figure 5.2, any rotation $\gamma$ of $\mathbf{V}$ around $\mathbf{D}$ defines right triangles with equal dimensions $D_{s}$, and $D_{m}$. Since their parameters are identical, all of these triangles are considered equal in the 2-D models of the previous chapter; if the effect of $\gamma$ is large, however, the 2-D moving-target model needs to be extended in order to be used effectively in 3-D.
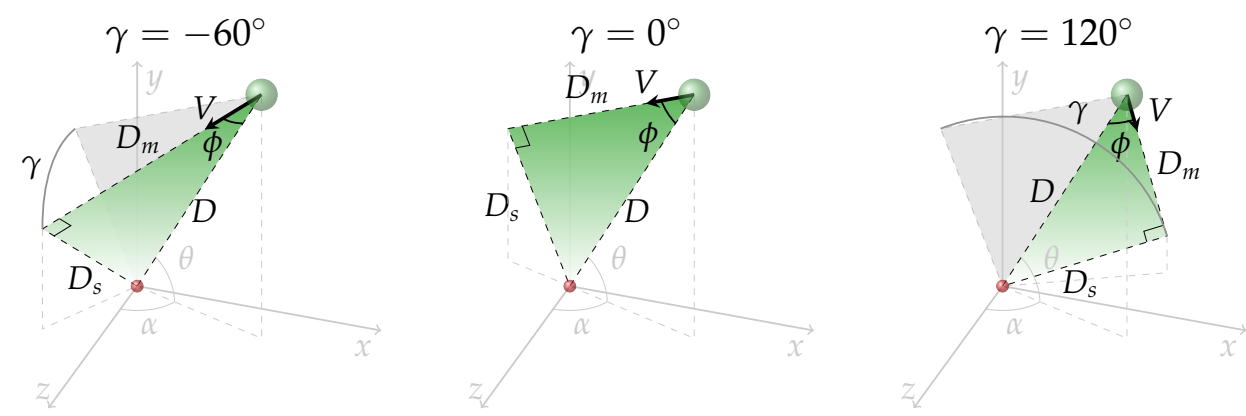

Figure 5.2. Identical model parameters $V, D_{m}$, and $D_{s}$ for three different $\gamma$ rotations of $\mathbf{V}$ around $\mathbf{D}$. 
Finally, to generalize the model to 3-D, the calculations of $D_{m}$, and $D_{s}$ can be simplified by using vector algebra. At any given instant, the displacement vector between the target's position $P$, and the user's wand position $P_{w}$ is given by

$$
\mathbf{D}^{\prime}=P_{w}-P .
$$

Given this, $D_{m}$, and $D_{s}$ correspond to the lengths of the projection and rejection of $\mathbf{D}^{\prime}$ on the target's unit velocity $\hat{\mathbf{V}}$, respectively, such that

$$
\begin{aligned}
D_{m} & =\left|\mathbf{D}^{\prime} \cdot \hat{\mathbf{V}}\right| \\
D_{s} & =\left\|\mathbf{D}^{\prime}-\left(\mathbf{D}^{\prime} \cdot \hat{\mathbf{V}}\right) \hat{\mathbf{V}}\right\| .
\end{aligned}
$$

Alternatively, the value of $D_{m}$ can be reused to calculate $D_{s}$, which can be less expensive computationally if the calculations are executed serially, such that

$$
D_{s}=\sqrt{\mathbf{D}^{\prime} \cdot \mathbf{D}^{\prime}-D_{m}^{2}}
$$

\subsection{Subjective difficulty and performance}

In Chapter 1 it was argued that prospective and performance assessments are related, but the former can complement or even supersede the latter because they are not dependent on action execution. Nonetheless, measures of performance, such as MT or percentage of errors are still important to evaluate users and interfaces objectively. Additionally, measuring the $P D$ of a given task requires either direct participant assessment, which interrupts the task, or the usage of sensors such as EEG [Kourtis et al. 2012], which may be invasive and are not available in all vR setups.

Based on the correlation between subjective difficulty (prospective, and perceived) and MT explored in previous work, it is hypothesized that the proposed PD formulae should also predict $M T$, such that

$$
M T=a_{V}+b_{V} \sqrt{D_{s}}+c_{V} \sqrt{D_{m}}+d_{V} \log _{2}\left(\frac{2 D_{m}}{W}\right) .
$$

The relation between $P D$ and $M T$, however, may be different for moving targets than for static ones. In particular, in Chapter 4 it was observed that $P D$ increases with $V$ even for targets that are moving toward the cursor, but in this case the MT should decrease with $V$ as long as the target has not been missed. This may imply that the signs in some of the regression coefficients in the proposed formulae for $P D$ may be contrary in MT formulae. Therefore, this chapter investigates the relation between the PD models of 2-D moving-target pointing introduced in Chapter 4, and the performance of 3-D moving-target selection tasks. 


\subsection{Predicting user intention in directed tasks}

Chapter 3 evaluated the possibility of predicting intention in undirected tasks using ID, based on the premise that users form their intentions by minimizing their prospective effort, as described by ID. In directed tasks, such as the ones studied in this chapter, the user must select the given target regardless of the prospective effort required to reach it. The principle of minimizing prospective effort, however, may still apply when choosing the path required to reach the specified target, similar to the way people choose routes in everyday life [Christenfeld 1995; Bailenson et al. 2000].

According to the initial segment strategy (ISs) [Christenfeld 1995; Bailenson et al. 2000], people tend to choose routes whose initial segments are straight, and where turns occur last-both characteristics are found in the model for 2-D moving-target selection proposed in Section 4.2.4. Therefore, the hypothesis that the target with the minimal $\widehat{M T}$ as predicted by Equation 5.5 is the intended target is evaluated in this chapter.

Since prediction is based only on one feature, a scoring function based on the one proposed by Ortega [2013], presented in Equation (2.23), is used. At each frame $t, \widehat{M T}$ is calculated for each of the $I$ targets using Equation (5.5). Targets are then ordered ascendingly by $\widehat{M T}$, their order given by $i=0, \cdots, I-1$. The score for each target $i$ is calculated following

$$
\begin{aligned}
& \widehat{T} \operatorname{Score}_{i}(t)=\widehat{T S C o r e}_{i}(t-1)+ \begin{cases}(N-i) \Delta t & \text { if } \quad i<N \\
-(\text { decay } \cdot N) \Delta t & \text { if } \quad i \geq N\end{cases} \\
& \widehat{T} \operatorname{Tcore}_{i}(t) \geq 0
\end{aligned}
$$

where $N$ is an arbitrary number of faster-to-reach targets, and decay is the rate with which scores decrease when a target $i \geq N$. In Ortega's studies with 100 spheres, a distance-based scoring function, $d \operatorname{Score}_{i}(t)$, was used with $N=20$, and decay $=0.5$, in Chapter $3, d \operatorname{Score}_{i}(t)$ was used with $N=1$, and decay $=0.9$. To validate the usefulness of $\widehat{T} S \operatorname{core}{ }_{i}(t)$, its predictive accuracy is compared to $d \operatorname{Score}_{i}(t)$

\subsection{Hypotheses}

The following hypotheses guided the design and analysis of the current study,

\subsubsection{Moving-target selection performance in 3-D}

H1. The three-part model with per-speed coefficients, and per-velocity intercept, described in Equation (5.5), accurately models the movement time of three-dimensional moving-target selection tasks. 
H1.1. The azimuth $\alpha$, altitude $\theta$, and $\gamma$ angles, not modeled in Equation (5.5), have a small effect on $M T$.

H1.2. In Equation (5.5) the coefficients $a_{V}, b_{V}, c_{V}$, and $d_{V}$ vary linearly with $V$.

\subsubsection{Predicting user intention in 3-D moving-target directed-selection tasks}

H2. At any given instant, the minimal movement time estimated by Equation (5.5) can be used to predict the intended target in a 3-D moving-target directed-selection task.

H2.1. The accuracy of the predictions based on estimated movement time will be greater than those based on wand-target distance.

These hypotheses assume univariate targets in a 3-D space, i.e., spheres.

\subsection{Methods}

\subsubsection{Participants}

Participants from Iowa State University were recruited through the Psychology Department research participant pool (sONA), through word of mouth, and through the HCI mailing list.

There were 33 participants, aged 18 to 39 years old $(M=23.48, M d n=21)$; ten participants were females, and only one participant was left handed. Most participants (31) were students, and nine participants reported having past experience in VR.

\subsubsection{Apparatus}

The user study took place in the Virtual Reality Applications Center, at Iowa State University. The code-base from Study 1 was reused and modified to implement this experiment. The VR JuggLua application was deployed in the C6, a six-surface CAVE-like virtual environment. All of the faces of the $3.05 \mathrm{~m}^{3} \mathrm{VE}$, except the back wall, were projected using active stereo at $4096 \times 4096 \mathrm{px}$. The participant's head and wand pose $(P, Q)$ were tracked using a 6-DOF Intersense Is-9oo inertial-ultrasonic hybrid tracker, allowing them to adequately perceive the 3-D world, and interact with it.

\subsubsection{Procedure}

Upon arriving to the study site, the participant was asked to read and accept a study consent form. If consent was granted, the participant was asked to answer a Qualtrics survey regarding their video game, vR, sports, and demographic background. The participant was also asked to complete the "Perspective Taking/Spatial Orientation Test" [Hegarty and Waller 2004], and the Simulator Sickness Questionnaire (ssQ) [Kennedy et al. 1993]. Subsequently, the participant's 
visual acuity was evaluated using the Interactive Visual Acuity Chart (IVAC) [Olitsky et al. 2006].

After completing the pre-survey questionnaire, the participant was asked to enter the C6 and step on a circular landmark located in the middle of the vE $(0,0,0)$, facing the front wall. After inquiring if the participant's perception of the virtual world was adequate, the experimenter asked the participant to choose a comfortable starting position for their wand $\left(P_{w, 0}\right)$, specifying that they should return to this position at the beginning of each trial.

In each trial, the participant was presented with an array of equally-textured virtual spheres of different sizes, initially located in front of them, and moving with different velocities. The spheres would start moving when the participant's wand was placed within a green translucent sphere, $s p h_{\underline{0}}$, of radius $0.05 \mathrm{~m}$ centered at $P_{w, \underline{0}}$.

The participant was asked to keep the wand within $s p h_{\underline{0}}$ until the goal sphere became highlighted. If they did not follow this instruction, $s p h_{\underline{0}}$ would turn red. Once the goal sphere became highlighted, $s p h_{\underline{0}}$ would disappear and the participant was asked to extend their arm to touch the goal sphere. If any sphere was touched during the selection process, whether highlighted or not, it disappeared. The trial, however, would end only when the highlighted sphere was touched, or if its distance to the user's head was greater than $2 \mathrm{~m}$, in which case it was assumed to be unreachable.

Visual and auditory feedback were used to indicate participant performance. A virtual counter was placed in front of the participant at $(-1,-1,-10)$, which showed the number of highlighted spheres missed by the participant during each experimental block. The counter would be reset to zero at the beginning of each block of trials. Two different sounds were played: one when the participant hit any sphere, and another when the trial ended due to the highlighted sphere being too far away, respectively.

At each frame, the elapsed time, the pose of the participant's head $\left(P_{h}, Q_{h}\right)$ and wand $\left(P_{w}, Q_{w}\right)$, each of the sphere positions $\left(P_{i}\right)$, and the possible wand-sphere collisions were recorded in a $\log$ file. The experimental setup is depicted in Figure 5.3.

After all the trials were complete, the participant was asked to exit the C6 and fill a post-experiment survey, which included the ssQ. The total length of the study was less than 60 minutes.

\subsubsection{Design}

A within-subjects, factorial design was used, with three blocks of trials, each with a different number of conditions presented in a random order without replacement.

In the first block there was one sphere per trial. ${ }^{1}$ The target was placed in one of the front vertices of an imaginary tetrahedron whose sides were $1 \mathrm{~m}$, with one vertex placed at $P_{w, \underline{0}}$, and the three others in front of the participant. Factors were sphere position, $P \in$

\footnotetext{
${ }^{1}$ Notice that participants were still instructed to stay at $P_{w, \underline{0}}$ before this sole sphere became highlighted.
} 
$\{$ up, bottom-left, bottom-right $\}$, radius, $r \in\{0.1,0.2\} \mathrm{m}$, speed, $V \in\{0.5,1,1.5\} \mathrm{m} / \mathrm{s}$, and initial movement direction, $\phi \in\{15,30,45\}^{\circ}$. Since there were already 54 conditions, the value of the initial $\gamma$ angle was set randomly between $180^{\circ}$, and $360^{\circ}$; there was one trial per condition, for a total of 1,782 trials.

In the second block there were three spheres per trial. The targets were placed in one of the front vertices of an imaginary tetrahedron whose sides were $1 \mathrm{~m}$, with one vertex placed at $P_{w, \underline{0}}$, and the three others in front of the participant. Factors were sphere position, $P \in\{$ up, bottom-left,bottom-right $\}$, radius, $r \in\{0.1,0.2\} \mathrm{m}$, speed, $V \in\{0.5,1,1.5\} \mathrm{m} / \mathrm{s}$, and initial movement direction, $\phi \in\{15,30,45\}^{\circ}$. The value of the initial $\gamma$ angle was set randomly between $180^{\circ}$, and $360^{\circ}$, and the $(P, r, V, \gamma)$ conditions of the two remaining spheres were also set randomly within the possible experimental values. There was one trial for each of the 54 conditions in this block, for a total of 1,782 trials.

In the last block there were six spheres per trial. The targets were placed in one of the front vertices of two imaginary tetrahedrons, each with sides of $1 \mathrm{~m}$, with one vertex placed at $P_{w, \underline{0}}$, and the three others in front of the participant; the front vertices of the second tetrahedron correspond to the vertically-mirrored positions of the first tetrahedron's front vertices (this arrangement is shown in Figure 5.3, middle). Factors were sphere position, $P \in\{$ up, up-left, bottom-left, bottom, bottom-right, up-right $\}$, radius, $r \in\{0.1,0.2\} \mathrm{m}$, speed, $V \in\{0.5,1,1.5\} \mathrm{m} / \mathrm{s}$, and initial movement direction, $\phi \in\{15,30,45\}^{\circ}$. The value of the initial $\gamma$ angle was set randomly between $180^{\circ}$, and $360^{\circ}$, and the $(P, r, V, \gamma)$ conditions of the five remaining spheres were also set randomly within the possible experimental values. There was one trial for each of the 108 conditions in this block, for a total of 3,564 trials.

Regardless of the block, in all trials the initial sphere positions were set at $P_{i}-\mathbf{V}_{i} * 1 \mathrm{~s}$. The goal sphere became highlighted after getting past its original tetrahedral position $P$, i.e. after approximately $1 \mathrm{~s}$, when approximately at $D=1 \mathrm{~m} .^{2}$ The criterion for highlighting the sphere was $D<1 \mathrm{~m}$. Notice that, at the moment of highlight all spheres were approximately at their tetrahedral position $P_{i}$, at about the same distance from $P_{w, \underline{0}}$. This was done to give participants some time to perceive the motion of the different spheres, and to make the sphere arrangements seemingly aleatory.

\subsection{General results}

\subsubsection{Experimental issues}

There were several technical issues, as well as some participant wellness problems during the execution of the experiment.

\footnotetext{
${ }^{2}$ These values are approximate due to the variable frame rate.
} 

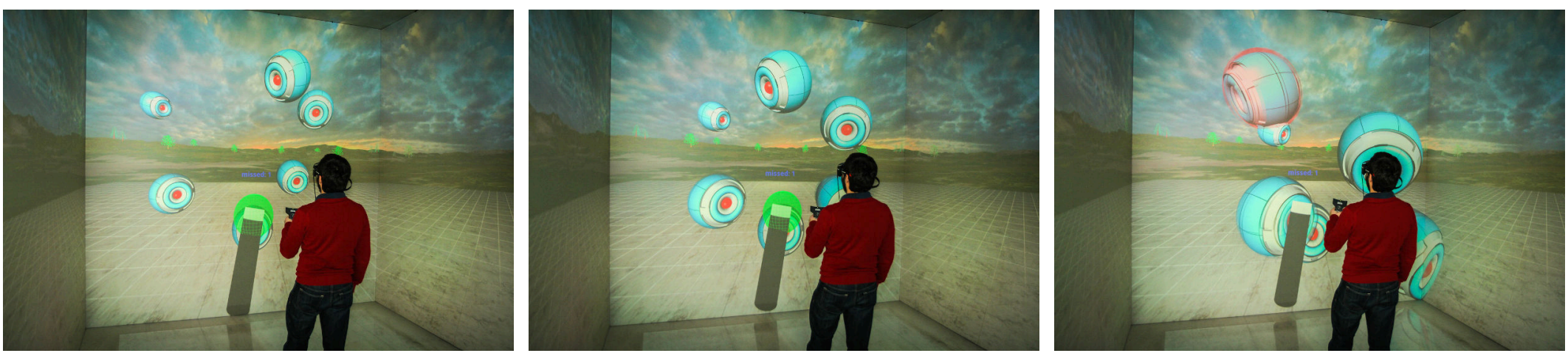

Figure 5.3. Experimental setup with six spheres. Left, the sphere starting positions, middle the spheres approximately at their controlled positions, right the spheres after the goal sphere gets highlighted. The green sphere represents $s p h_{\underline{0}}$.

\subsubsection{Technical issues}

Incomplete logging. Because of logging problems, the data from the first two participants was incomplete, so they were excluded from the performance and intention analyses.

Tracking interruptions. In some trials, the wand tracker lost track of the participant's wand causing a delay between the real and the virtual wand or, in the worst case scenario, a complete cessation of the tracking. In the latter cases, the experimenter asked the participants to stop the motion of the wand to allow the tracking to resume. This was most noticeable on participants with brusque movements. Fortunately, by virtue of the experimental design, in which trials would not start before the participant placed the wand at $s p h_{\underline{0}}$, this issue did not affect consecutive trials. Finally, in several trials the head tracker stopped functioning, an issue that was most often detected and fixed at the end of each block; nonetheless, the participants did not seem to notice this issue. No trials were removed from the data due to tracking interruptions.

Projector problems. During five experimental sessions, one or two of the C6 projectors could not be started. In three instances the affected projector was in the right screen, in one instance the affected projector belonged to the front screen, and in the last instance both the right and front faces were affected. These sessions were, nonetheless, included in the analyses.

\subsubsection{Participant-wellness issues}

Two participants had noticeable wellness problems. The first of these participants had a cast on their right arm upon arrival, but decided to participate using with their left hand. The second participant decided to take a break during the three-sphere block, and declared having some discomfort on their right shoulder; even though the experimenter suggested to terminate the session without any consequences for the participant, they decided to continue 
using their left hand. This last participant commented having some nausea by the end of the experiment. These sessions were retained in the analyses.

\subsubsection{Simulator sickness questionnaire}

The SSQ ratings were scored using the procedure described by Kennedy et al. [1993, p. 212]. The results, shown in Figure 5.4, show that the score distributions assessed before and after the experiment are very similar. Notice that each of the categories, nausea, oculomotor, disorientation, as well as the total score, have different scales.

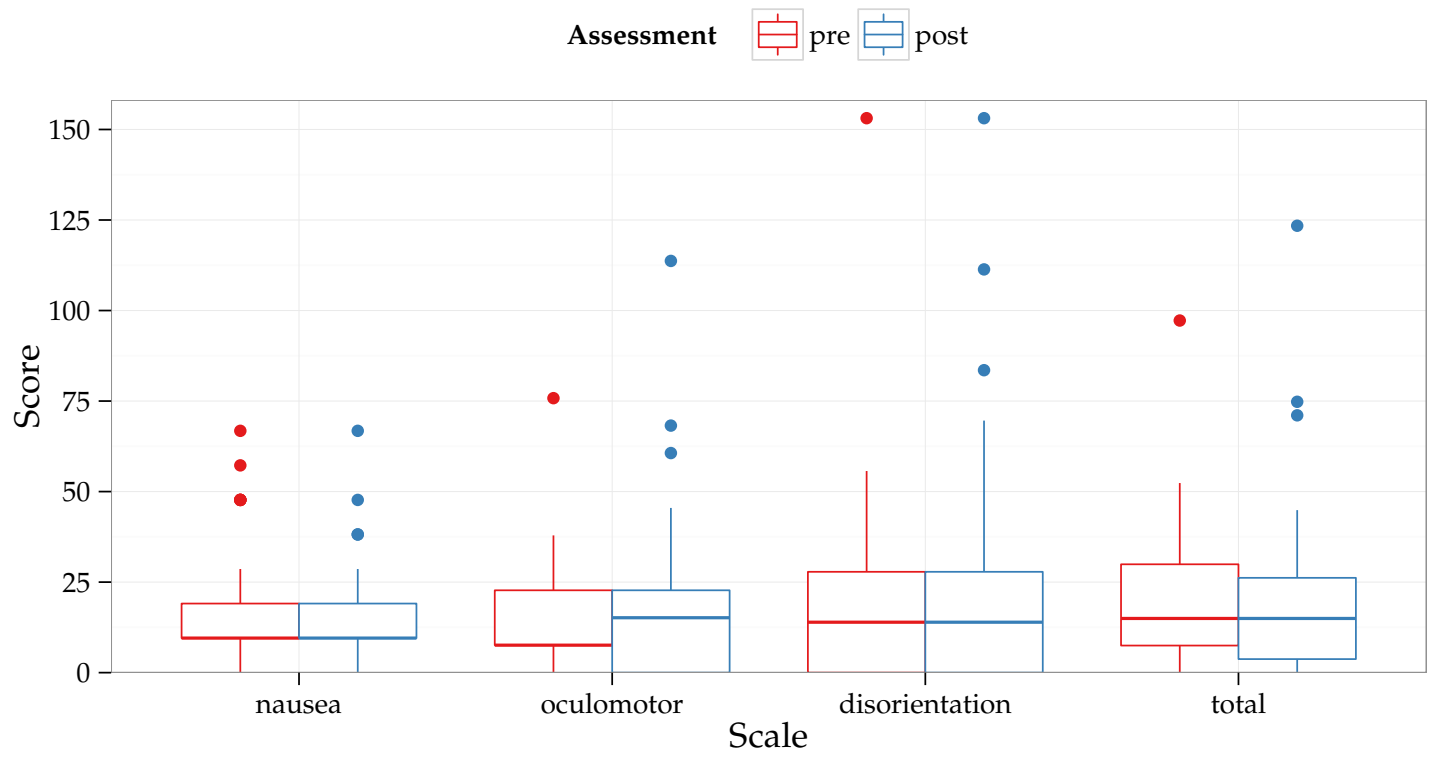

Figure 5.4. Distributions of the pre- and post-experiment assessment scores of the ssQ by scale. Lower and upper lines of the boxes represent the first and third quantiles, their distance called the inter-quantile range (IQR), thick box lines represent the median values, upper and lower whiskers represent values that are within $1.5 \mathrm{IQR}$ of the box hinges. Points represent values that are greater than the third quantile plus $1.5 \mathrm{IQR}$.

Even though the main goal of this study is not to explore simulator sickness, these results give some evidence against a possible effect of simulator sickness in trial performance and participant action. This evidence, however, contradicts the conjecture that simulator sickness is worsened in moving-target selection presented in Chapter 1. 


\subsection{Performance analysis}

\subsubsection{Exploratory data analysis}

In accordance with some of the ideas of Tukey [1980], a brief exploratory analysis is carried out before attempting to apply statistical-modeling techniques to summarize the data. This analysis serves the purpose of presenting the general trends in the data, informally assessing the aforementioned hypotheses, and possibly unveiling (or letting the reader discover) unexpected findings. Following Tukey's [1980, p. 24] statement that "the picture-examining eye is the best finder we have of the wholly unanticipated," this analysis is mostly carried out using data visualization techniques; numerical methods to quantify the magnitude and uncertainty of the different effects are instead presented in the subsequent section.

\subsubsection{Between-block trial performance}

As shown on the left Figure 5.5, the percentage of successful captures $\rho$ per participant decreased with the increase of Spheres per block. An effect of fatigue is discarded, given the similarity between the pre- and post-experiment scores of the SSQ presented above.

It is likely, however, that this difference is due to a delayed reaction time $R T$ as the number of spheres increased, which is congruent with the Hick-Hyman law [Hick 1952; Hyman 1953]. This is consistent with the increase in $T$ that follows the increment of spheres per block displayed on the right of Figure 5.5.
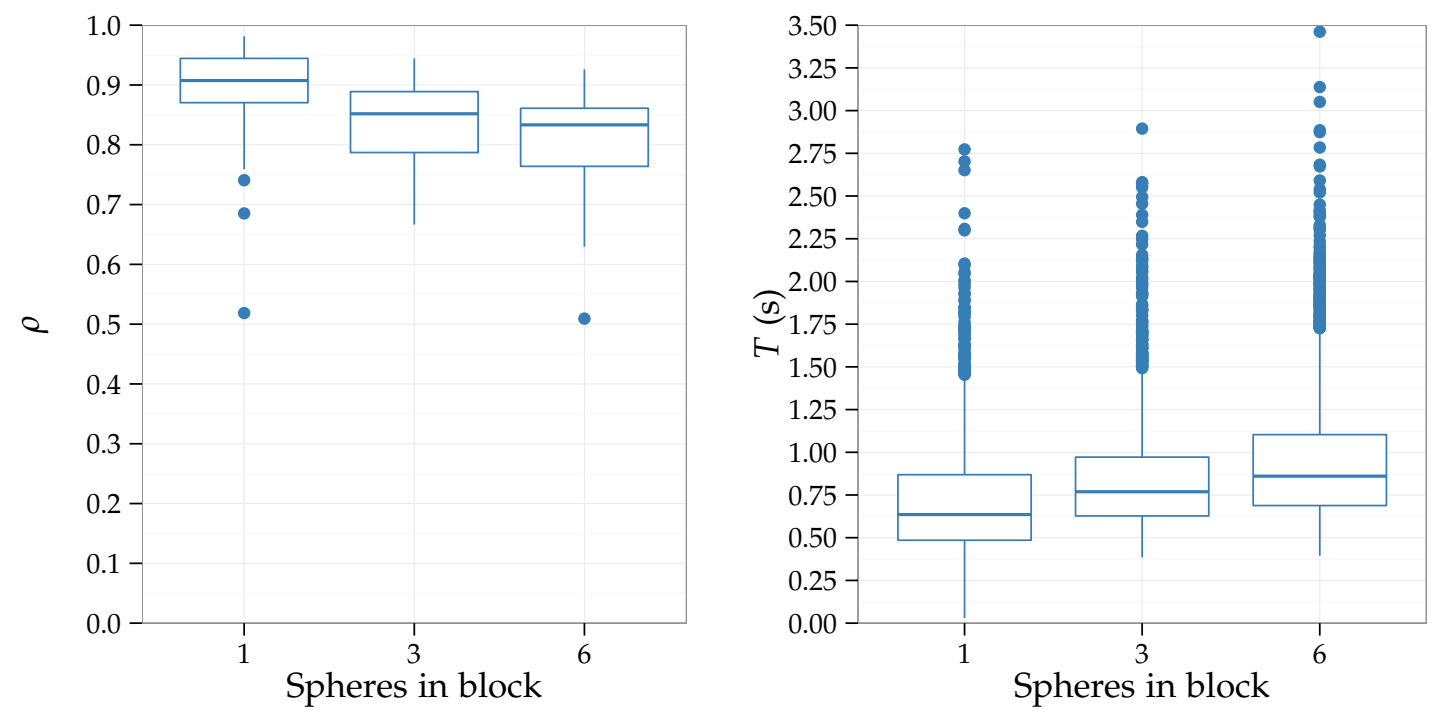

Figure 5.5. Distribution of participant success rates (left), and successful trial completion times (right) per experimental block.

$T$ is used as a proxy for $R T$, which cannot be measured directly from the data. This 
substitution is not perfect since participants may have selected other spheres prior to attaining the target sphere in trials where the number spheres was greater than one. In the 1-sphere trials, additionally, some participants may have "cheated" by approaching the target sphere before it was highlighted, which corresponds to the values of $T$ close to zero at the tip of the lower whisker of the 1-sphere $T$ boxplot.

\subsubsection{Effects on MT for the successful trials in the 1-sphere block}

It is clear that the number of spheres per trial affected participant performance. However, the main goal of the current section, in terms of performance prediction, is to identify the factors that affect the MT required to reach a single moving target. Therefore, the scope of this analysis covers only the successful trials of the 1-sphere block; the extension to multiple targets is left for future work.

In this section, a distinction is made between controlled factors, i.e., $P, r, V$, and $\phi$ that were systematically assigned to each trial, and experimental covariates, i.e., factors that were either assigned randomly, or not controlled for during each trial.

Controlled factors. Among the main factors, $V$ had the strongest effect on the MT of the successful trials, as shown in Figure 5.6. As expected, MT decreased with the increase of $V$. In low velocity trials, additionally, the span of $M T$ was also larger.

Target radius $r$ also had a visible effect on MT. Over all, bigger targets had shorter MT, although this decrease is visibly smaller for targets with high $V$, suggesting an interaction between both. Additionally, in low $V$ trials, the span of $M T$ was smaller for targets with $r=0.2$, than for targets with $r=0.1$.

Targets whose position $P$ was up with respect to $s p h_{\underline{0}}$ had larger MT than targets located below $s p h_{\underline{0}}$. This increase in MT was smaller for targets with high $V$, suggesting an interaction between $P$, and $V$.

Increase in $\phi$ resulted only in small increases of $M T$. In general, this increase was more visible for small targets located above $s p h_{\underline{0}}$.

Experimental covariates. Due to the experimental design, there are certain factors that were not controlled for, which could have an effect on $M T$, including $\gamma$, and the initial wand position at the highlight frame in each trial.

The effect of $\gamma$ on MT. To avoid generating too many experimental conditions, the angle $\gamma$ was randomly assigned between $180^{\circ}$ and $360^{\circ}$. Yet, in order to generalize the 2-D model to 3-D without further modification, its effect on MT must remain small.

As seen at the top of Figure 5.7, the distribution of angles is close to uniform in the $[180,360]^{\circ}$ range. As highlighted by the superimposed smoothing curve, on average, MT remained very similar across the range of assigned $\gamma$ angles. This suggests a very small effect of $\gamma$ on MT, which supports the usage of the 2-D model for 3-D tasks. 


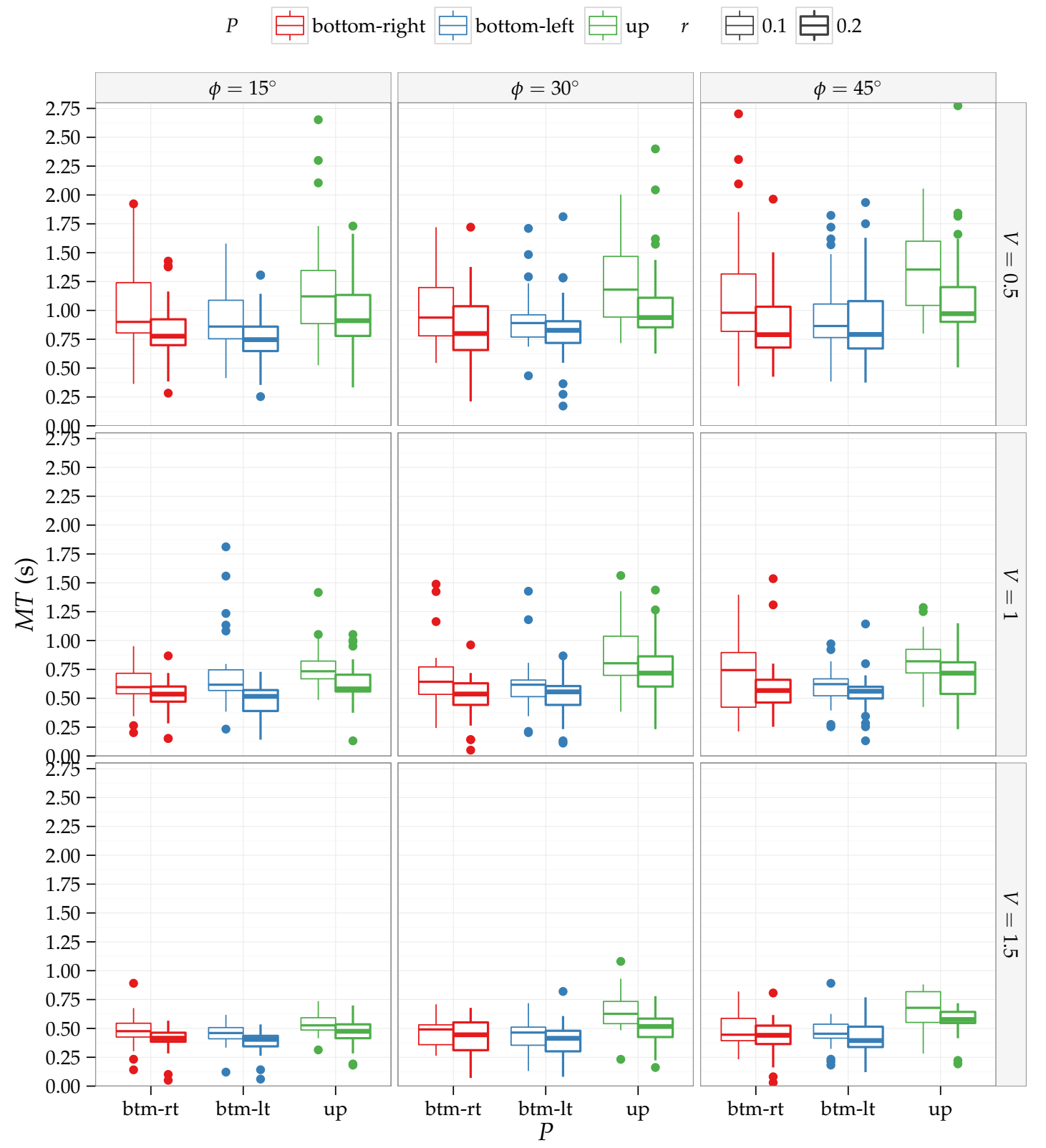

Figure 5.6. Distributions of movement times for the successful trials per experimental factor in the 1-sphere block. 

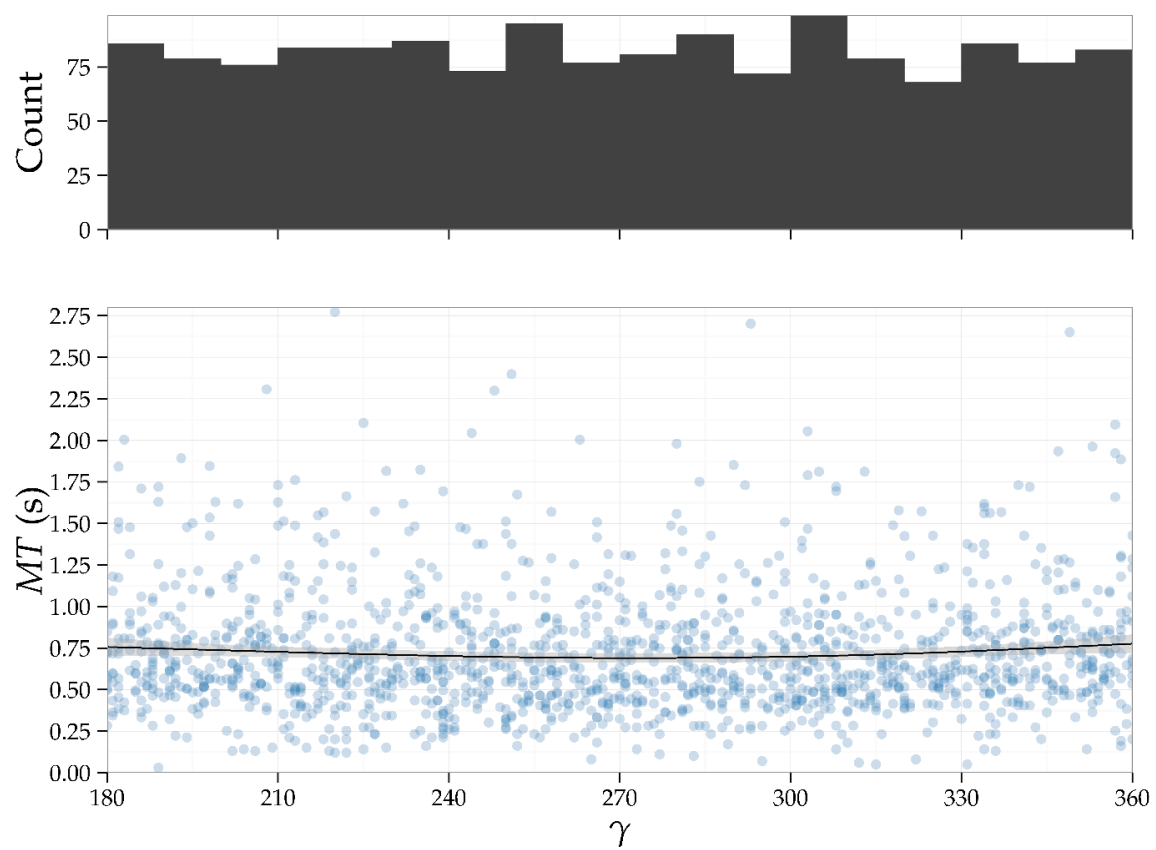

Figure 5.7. Movement times per $\gamma$ angle for the successful trials in the 1-sphere block. The top bars show the distribution of $\gamma$ binned every $10^{\circ}$.

Wand positions at the highlight frame. To avoid hindering the flow of the study, only a soft constraint was placed on the wand position before the highlight frame, i.e., $s p h_{0}$ which would change from green to red whenever it did not encircle the tip of the wand. Even though this allowed participants to approach the target sphere before it became highlighted, the three position density plots of Figure 5.8 show that the highest density of wand positions was within $s p h_{0}, p\left(\left\|P_{w}-P_{w, \underline{0}}\right\|<0.05\right)=0.5$. In a few trials, however, participants seem to have "cheated" by placing their wand beyond $s p h_{\underline{0}}$, thus approaching the target, $p\left(P_{w, z}-z_{\underline{0}}<\right.$ $-0.05)=0.17$. Similarly, the negative skew of the wand positions along the $y$ axis reflects the fact that in some trials participants got closer to the bottom targets by lowering their wands below $s p h_{\underline{0}}$; this phenomenon was less visible for trials were the target was initially above the user. Finally, the distribution of wand positions along the $x$ axis, shown on the left part of Figure 5.8, was symmetrical with respect to $x_{\underline{0}}$. The fact that participants were more compliant with their left-right and up positions relative to $P_{w, \underline{0}}$ may reflect a problem of depth perception and occlusion of the wand tip by $s p h_{\underline{0}}$.

The effect of the different $P_{w}$ at the highlight frame on each trial's MT can be seen in Figure 5.9. Overall, an increase in the wand-sphere distance $\left\|P_{w}-P\right\|$ resulted in an increase of about $0.88 \mathrm{~s}$ in $M T$, as highlighted by the trend line. This implies that even within the bounds of $s p h_{0}$, depicted by the vertical orange lines, the average change in MT is about $0.09 \mathrm{~s}$. The trend line was purposely chosen as a least-squares linear model to represent the approximate 

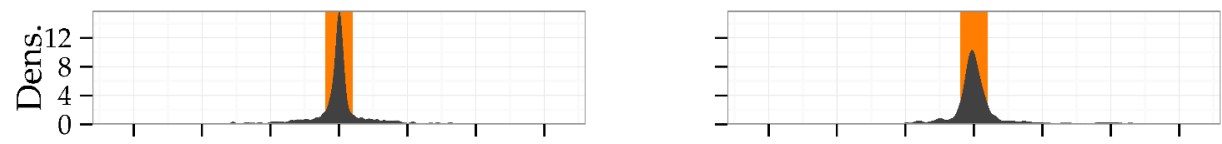

$P$

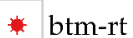

* btm-lt

* up
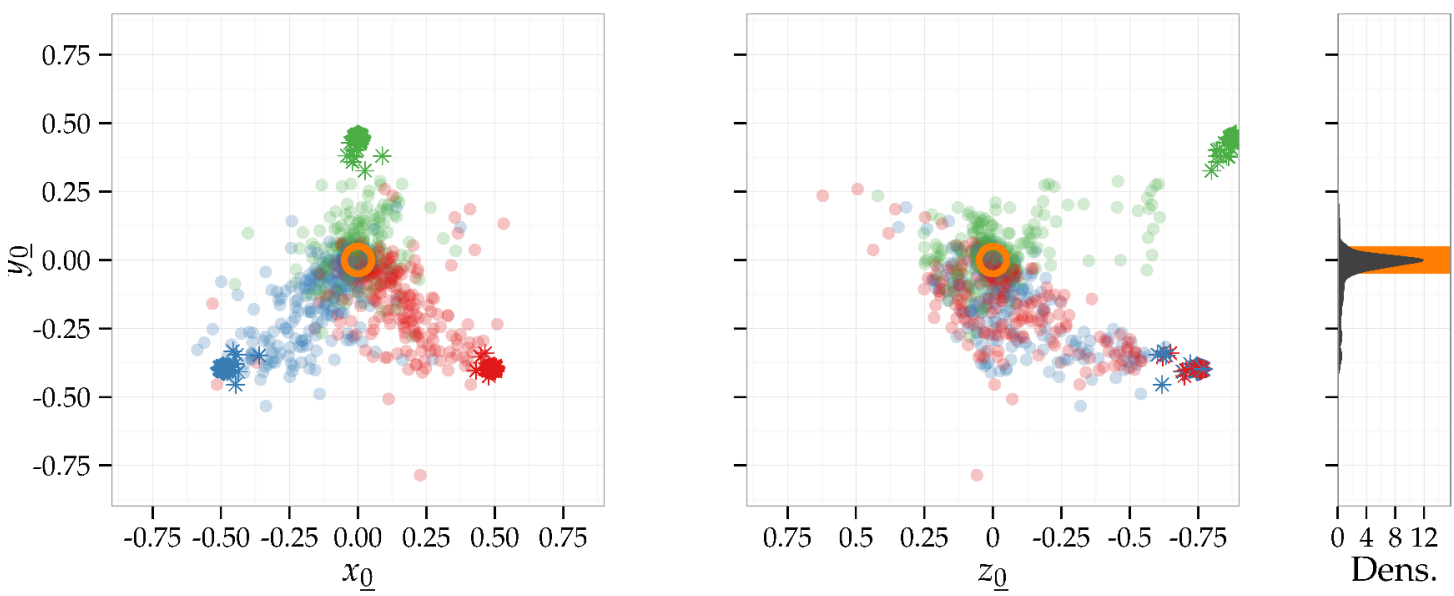

Figure 5.8. Front and right views of the distribution of wand positions at the frame where the sphere was highlighted in the 1-sphere trials. Red, blue, and green points represent the wand positions in trials where the sphere was at the bottom-right, bottom-left, and up, respectively. Correspondingly colored stars represent the sphere positions in those frames. The orange circle represents $s p h_{0}$. The top, and side black ribbons represent the densities of the distributions of $x_{\underline{0}}$ and $z_{\underline{0}}$, and $y_{\underline{0}}$, overlayed on the length of $s p h_{\underline{0}}$ along that coordinate shown in orange. Coordinates are relative to $P_{w, \underline{0}}$. 
increase in $M T$ per $\left\|P_{w}-P\right\|$, but it is clear that such a simple model would yield poor $M T$ predictions. $^{3}$
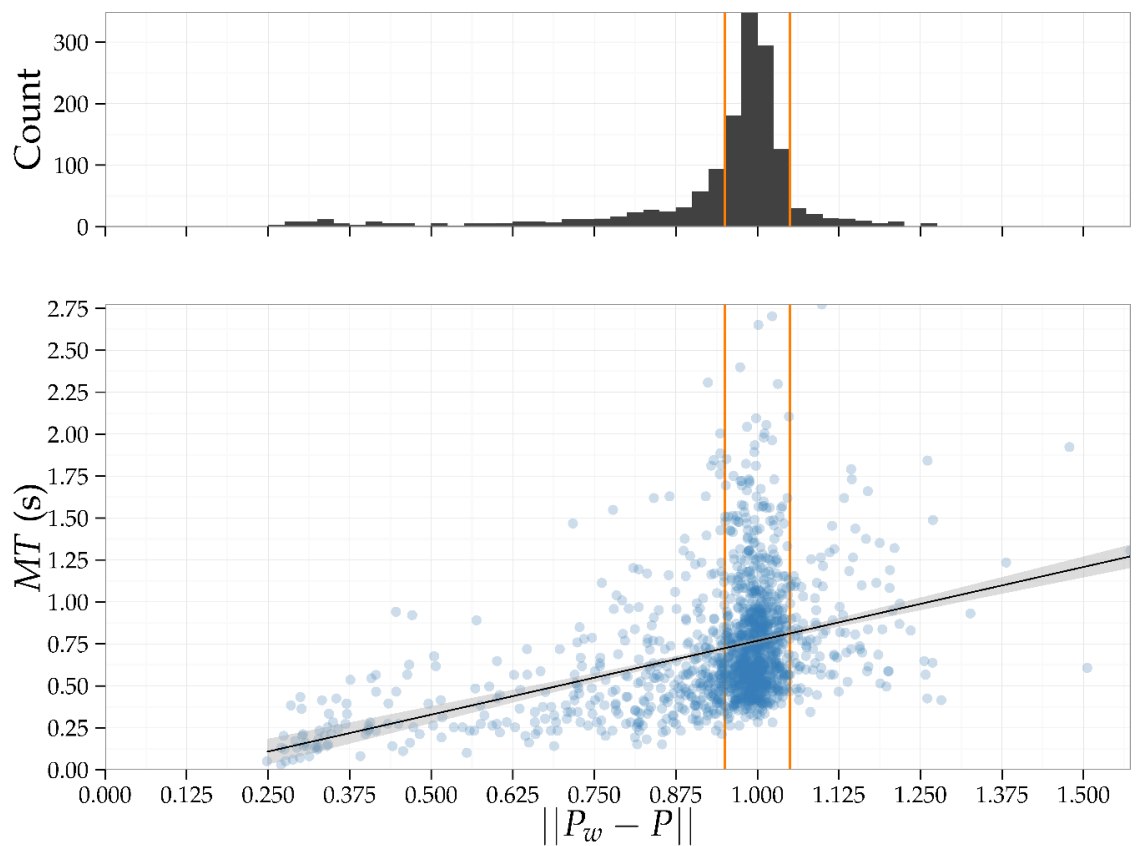

Figure 5.9. Movement times for each of the distances between the target sphere and the wand at the highlight frame $\left\|P_{w}-P\right\|$, for the successful trials in the 1-sphere block. The top bars show the distribution of $\left\|P_{w}-P\right\|$ binned every $0.025 \mathrm{~m}$. The orange lines represent the distance between the target sphere and $P_{w, \underline{0}} \pm 0.05 \mathrm{~m}$.

\subsubsection{Regression analysis}

Successful trials in the 1-sphere block are grouped by $(P, r, V, \phi)$ condition. In accordance with the typical Fitts literature, each condition's MT is summarized using means. Due to their variation with respect to the controlled conditions, the wand and sphere positions per condition are set as their mean at the highlight frame, i.e., $\bar{P}_{w}=\left(\bar{P}_{w, x}, \bar{P}_{w, y}, \bar{P}_{w, z}\right)$, and $\bar{P}=\left(\bar{P}_{x}, \bar{P}_{y}, \bar{P}_{x}\right)$. Using $\bar{P}_{w}$, and $\bar{P}, D_{m}$, and $D_{s}$ are calculated using Equations (5.2), and (5.4).

Following Equation (5.5), a linear regression of the form

$$
\overline{M T} \sim \mathcal{N}\left(a_{V}+b_{V} \sqrt{D_{s}}+c_{V} \sqrt{D_{m}}+d_{V} I D_{m}, \sigma^{2}\right)
$$

is fit. The resulting estimates, presented in Table 5.1, show a very good fit in terms of $R^{2}$, with credible values in $[0.89,0.96]$. The distributions of $a, b, c$, and $d$ decrease in magnitude

\footnotetext{
${ }^{3}$ Such a linear model would suffer from several deficiencies, which includes explaining only a small percentage of the variance of $M T\left(R^{2}=0.15\right.$, portrayed by the narrow confidence interval compared to the span of the data [Gelman and Hill 2007, p. 62]), and the clearly heterogeneous variance in the residual distribution.
} 
with $V$, and the non-intercept parameter with highest magnitude is $c$, potentially indicating the importance of the ballistic component of the movement along the target trajectory on MT. The regression is represented graphically in Figure 5.10.

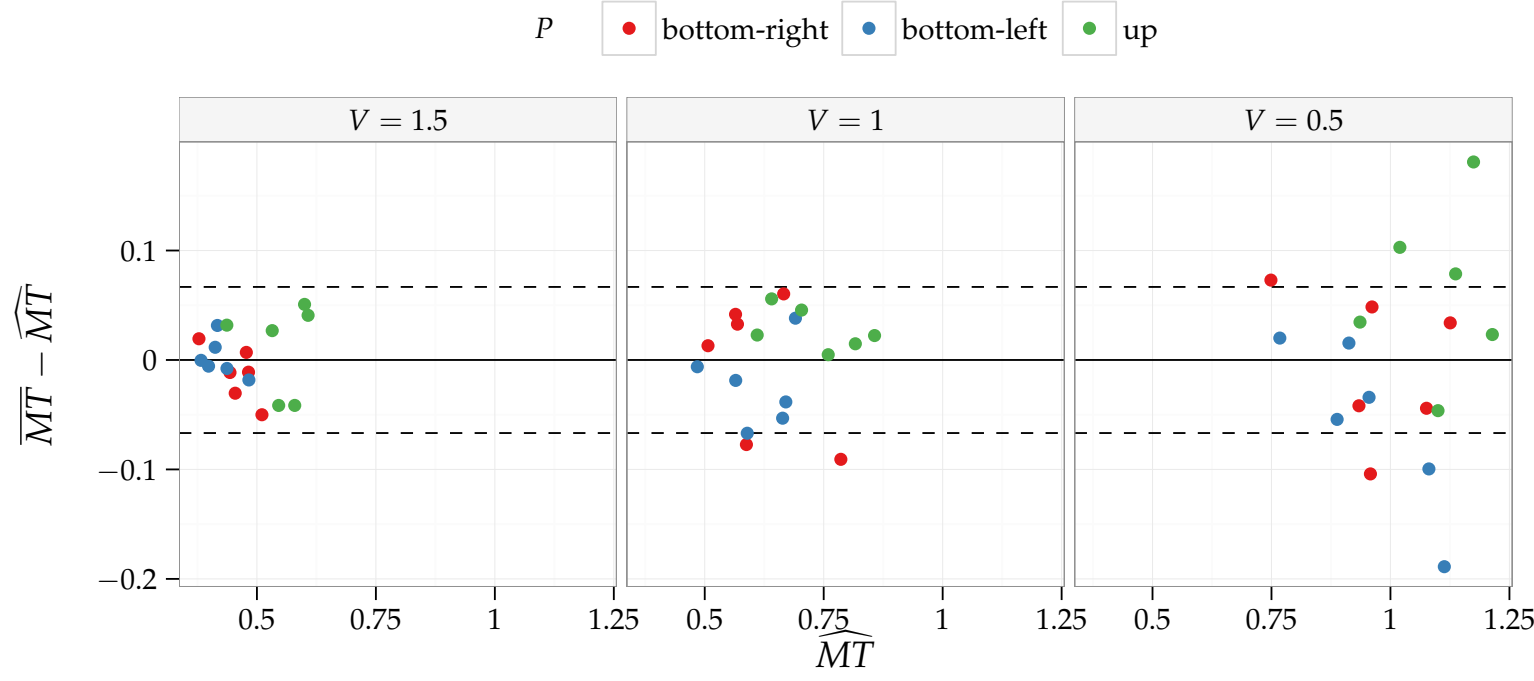

Figure 5.10. Residuals $(\overline{M T}-\widehat{M T})$ vs. fitted values $(\widehat{M T})$ for the $\overline{M T} \sim \mathcal{N}\left(a_{V}+b_{V} \sqrt{D_{s}}+c_{V} \sqrt{D_{m}}+d_{V} I D_{m}, \sigma^{2}\right)$ regression. The dotted lines represent $\pm \hat{\sigma}$.

The residual plot of the regression, presented in Figure 5.10, suggests that the residual variance increases with the decrease in $V$. This implies that $\overline{M T}$ predictions for low speed targets, $V=0.5$, will be less accurate than predictions for high speed targets, $V=1.5$.

Such a result is consistent with existing theories on moving target interception, which suggest that tasks with less temporal constraints (e.g., when targets have low $V$ ) are more prone to sensory-motor noise, thus resulting in more variable MT [Tresilian 2005, 134-135]. Therefore, given that the lack of precision in $\overline{M T}$ predictions for lower $V$ may be due to irreducible noise, and that there are only two visible outliers, the model is not further modified.

\subsubsection{Change of coefficients per $V$}

Consistently with the $P D$ analyses from Chapter 4, and the gain analyses of Shoemaker et al. [2012], linear regressions on the per- $V$ parameter estimates from Table 5.1- $\hat{a}_{V}=M d n\left(a_{V}\right)$, $\hat{b}_{V}=M d n\left(b_{V}\right), \hat{c}_{V}=M d n\left(c_{V}\right)$, and $\hat{d}_{V}=M d n\left(d_{V}\right)$-are first calculated using the default least-squares method in $\mathrm{R}$ [2015]. The results, shown in Table 5.2, give some evidence in support of the linear relation between $V$ and $\hat{a}, V$ and $\hat{b}, V$ and $\hat{c}$, and, to a lesser degree $V$ and $\hat{d}$. 
Table 5.1. Regression estimates for $\overline{P D}=a_{V}+b_{V} \sqrt{D_{s}}+c_{V} \sqrt{D_{m}}+d_{V} I D_{m}$.

\begin{tabular}{ccc} 
Estimand & $M d n$ & $95 \%$ HDI \\
\hline$a_{V=0.5}$ & -3 & {$[-4.75,-1.21]$} \\
$a_{V=1.0}$ & -2.48 & {$[-3.93,-1.04]$} \\
$a_{V=1.5}$ & -1.82 & {$[-3.14,-0.51]$} \\
$b_{V=0.5}$ & 1.25 & {$[0.83,1.67]$} \\
$b_{V=1.0}$ & 1.03 & {$[0.61,1.46]$} \\
$b_{V=1.5}$ & 0.83 & {$[0.4,1.24]$} \\
$c_{V=0.5}$ & 3.09 & {$[1.36,4.76]$} \\
$c_{V=1.0}$ & 2.47 & {$[1.07,3.88]$} \\
$c_{V=1.5}$ & 1.74 & {$[0.54,2.94]$} \\
$d_{V=0.5}$ & 0.16 & {$[0.1,0.23]$} \\
$d_{V=1.0}$ & 0.11 & {$[0.04,0.17]$} \\
$d_{V=1.5}$ & 0.09 & {$[0.03,0.16]$} \\
$\sigma$ & 0.07 & {$[0.05,0.08]$} \\
$R^{2}$ & 0.93 & {$[0.89,0.96]$}
\end{tabular}

Table 5.2. Least-squares estimates for the regressions of the point estimates from Table $5.1-\hat{a}_{V}=\operatorname{Mdn}\left(a_{V}\right), \hat{b}_{V}=\operatorname{Mdn}\left(b_{V}\right), \hat{c}_{V}=\operatorname{Mdn}\left(c_{V}\right)$, and $\hat{d}_{V}=\operatorname{Mdn}\left(d_{V}\right)$ - on target speed $V$.

\begin{tabular}{ccccc} 
Model & Intercept & Slope & $\hat{\sigma}$ & $\hat{R}^{2}$ \\
\hline$\hat{a}_{V} \sim \mathcal{N}\left(\alpha_{1}+\alpha_{2} V, \sigma_{a}^{2}\right)$ & -3.62 & 1.19 & 0.06 & 0.99 \\
$\hat{b}_{V} \sim \mathcal{N}\left(\beta_{1}+\beta_{2} V, \sigma_{b}^{2}\right)$ & 1.46 & -0.42 & 0.01 & 1 \\
$\hat{c}_{V} \sim \mathcal{N}\left(\zeta_{1}+\zeta_{2} V, \sigma_{c}^{2}\right)$ & 3.78 & -1.35 & 0.04 & 1 \\
$\hat{d}_{V} \sim \mathcal{N}\left(\delta_{1}+\delta_{2} V, \sigma_{d}^{2}\right)$ & 0.19 & -0.07 & 0.02 & 0.79
\end{tabular}

As discussed in Section 4.4.2.2, the least-squares estimates on regression coefficients tend to be too optimistic. Thus, to better assess the uncertainty around the estimates of Table 5.2, a hierarchical model which simultaneously estimates the latter parameters and those in Table 5.1, is fit. The hierarchical model reuses the least-squares estimates $\hat{\alpha}_{1}, \hat{\alpha}_{2}, \hat{\beta}_{1}, \hat{\beta}_{2}, \hat{\zeta}_{1}, \hat{\zeta}_{2}$, $\hat{\delta}_{1}$, and $\hat{\delta}_{2}$ from Table 5.2 as priors for the mean parameters of $\alpha_{1}, \alpha_{2}, \beta_{1}, \beta_{2}, \zeta_{1}, \zeta_{2}, \delta_{1}$, and $\delta_{2}$, respectively; the wide prior variance $\left(2^{2}\right)$, with respect to the range of $\overline{M T}(0.38-1.36)$, allows for some flexibility both in the mean and the variance of the marginal posteriors. Given the low number of levels of $\#(V)=3$, and following the recommendations of Gelman [2006] for standard deviation parameters on hierarchical models, half-Cauchy priors are chosen for $\sigma_{a}$, $\sigma_{b}, \sigma_{c}, \sigma_{d}$, and $\sigma$, with scale parameter $A=1$, which, again, is wide with respect to the range of $\overline{M T}$.

The model is fit using Stan [2015], with four MCMC chains of 10,000 iterations, including 5,000 warm-up iterations, for a total of 20,000 saved iterations. The levels and estimates of the model shown in Table 5.3, show higher estimates for $\sigma_{a}, \sigma_{b}, \sigma_{c}$, and $\sigma_{d}$ than those obtained in 
Table 5.2, and correspondingly, wider intervals around $\hat{\alpha}_{1}, \hat{\alpha}_{2}, \hat{\zeta}_{1}, \hat{\zeta}_{2}, \hat{\delta}_{1}$, and $\hat{\delta}_{2}$ than those that would be obtained from the least-squares regression. Nevertheless, the minimal change in the distributions of $a, b, c, d$, and $\sigma$ between Tables 5.1 and 5.3 suggests that, in spite of the wide uncertainty, the linear relations between $V$ and $a, b, c$, and $d$ are plausible and congruent with the data.

Table 5.3. Levels and estimates of the hierarchical regression $\overline{M T}_{i} \sim \mathcal{N}\left(a_{V[i]}+b_{V[i]} \sqrt{D_{s i}}+c_{V[i]} \sqrt{D_{m i}}+d_{V[i]} I D_{m i}, \sigma^{2}\right) . \quad \hat{\alpha}_{1}, \hat{\alpha}_{2}, \hat{\beta}_{1}, \hat{\beta}_{2}, \hat{\zeta}_{1}$, $\hat{\zeta}_{2}, \hat{\delta}_{1}$, and $\hat{\delta}_{2}$ correspond to the least-squares estimates from Table 5.2.

\begin{tabular}{|c|c|c|c|}
\hline Model & Estimand & $M d n$ & 95\% HDI \\
\hline \multirow{3}{*}{$a_{V} \sim \mathcal{N}\left(\alpha_{1}+\alpha_{2} V, \sigma_{a}^{2}\right)$} & $a_{V=0.5}$ & -3.08 & {$[-4.26,-1.93]$} \\
\hline & $a_{V=1.0}$ & -2.48 & {$[-3.39,-1.64]$} \\
\hline & $a_{V=1.5}$ & -1.84 & {$[-2.94,-0.75]$} \\
\hline \multirow{3}{*}{$b_{V} \sim \mathcal{N}\left(\beta_{1}+\beta_{2} V, \sigma_{b}^{2}\right)$} & $b_{V=0.5}$ & 1.27 & {$[0.96,1.59]$} \\
\hline & $b_{V=1.0}$ & 1.04 & {$[0.75,1.31]$} \\
\hline & $b_{V=1.5}$ & 0.83 & {$[0.48,1.2]$} \\
\hline \multirow{3}{*}{$c_{V} \sim \mathcal{N}\left(\zeta_{1}+\zeta_{2} V, \sigma_{c}^{2}\right)$} & $c_{V=0.5}$ & 0.16 & {$[0.1,0.22]$} \\
\hline & $c_{V=1.0}$ & 0.11 & {$[0.05,0.17]$} \\
\hline & $c_{V=1.5}$ & 0.09 & {$[0.03,0.16]$} \\
\hline \multirow{3}{*}{$d_{V} \sim \mathcal{N}\left(\delta_{1}+\delta_{2} V, \sigma_{d}^{2}\right)$} & $d_{V=0.5}$ & 0.16 & {$[0.1,0.22]$} \\
\hline & $d_{V=1.0}$ & 0.11 & {$[0.05,0.17]$} \\
\hline & $d_{V=1.5}$ & 0.09 & {$[0.03,0.16]$} \\
\hline$\sigma \sim$ half-Cauchy $(1)$ & $\sigma$ & 0.07 & {$[0.05,0.08]$} \\
\hline \multirow{2}{*}{$\alpha_{j} \sim \mathcal{N}\left(\hat{\alpha}_{j}, 2^{2}\right)$} & $\alpha_{1}$ & -3.7 & {$[-5.69,-1.76]$} \\
\hline & $\alpha_{2}$ & 1.22 & {$[-0.47,2.98]$} \\
\hline$\sigma_{a} \sim \operatorname{half-Cauchy}(1)$ & $\sigma_{a}$ & 0.28 & {$[0,1.34]$} \\
\hline \multirow{2}{*}{$\beta_{j} \sim \mathcal{N}\left(\hat{\beta}_{j}, 2^{2}\right)$} & $\beta_{1}$ & 1.48 & {$[0.21,2.71]$} \\
\hline & $\beta_{2}$ & -0.44 & {$[-1.57,0.77]$} \\
\hline$\sigma_{b} \sim \operatorname{half-Cauchy}(1)$ & $\sigma_{b}$ & 0.2 & {$[0,1.14]$} \\
\hline \multirow{2}{*}{$\zeta_{j} \sim \mathcal{N}\left(\hat{\zeta}_{j}, 2^{2}\right)$} & $\zeta_{1}$ & 3.86 & {$[1.99,5.8]$} \\
\hline & $\zeta_{2}$ & -1.39 & {$[-3.14,0.27]$} \\
\hline$\sigma_{c} \sim$ half-Cauchy $(1)$ & $\sigma_{c}$ & 0.28 & {$[0,1.36]$} \\
\hline \multirow{2}{*}{$\delta_{j} \sim \mathcal{N}\left(\hat{\delta}_{j}, 2^{2}\right)$} & $\delta_{1}$ & 0.19 & {$[-0.74,1.19]$} \\
\hline & $\delta_{2}$ & -0.07 & {$[-0.96,0.82]$} \\
\hline$\sigma_{d} \sim \operatorname{half-Cauchy}(1)$ & $\sigma_{d}$ & 0.11 & {$[0,0.86]$} \\
\hline
\end{tabular}

\subsection{Predictive analysis}

The $\widehat{T} S \operatorname{core}_{i}(t)$ scoring function used in this section is calculated using the $\widehat{M T}$ predictions given by Equation 5.5 with the parameter estimates from Table 5.1. 


\subsubsection{Three-sphere trials}

\subsubsection{Wand positions at the highlight frame}

Before attempting to predict the intended sphere in each trial, the wand positions at the highlight frame are visualized to identify potential issues, and differences with the 1-sphere trials, that could affect the predictions.

As shown in Figure 5.11, compared to the 1-sphere block of trials, there was a larger percentage of wand positions within $s p h_{\underline{0}}$ at the highlight frame, $p\left(\left\|P_{w}-P_{w, 0}\right\|<.05\right)=0.64$. "Cheating" was not possible in this block since the goal sphere was unknown before it became highlighted, as opposed to the 1-sphere block. Thus, as shown in the top right, and rightmost distributions of Figure 5.11, instead of placing their wand beyond $s p h_{0}$, participants who did not have their wand within $s p h_{\underline{0}}$ tended to place their wand closer to their bodies, $p\left(P_{w, z}-z_{\underline{0}}>.05\right)=0.22$, and below $s p h_{\underline{0}}, p\left(P_{w, y}-y_{\underline{0}}<-.05\right)=0.13$.
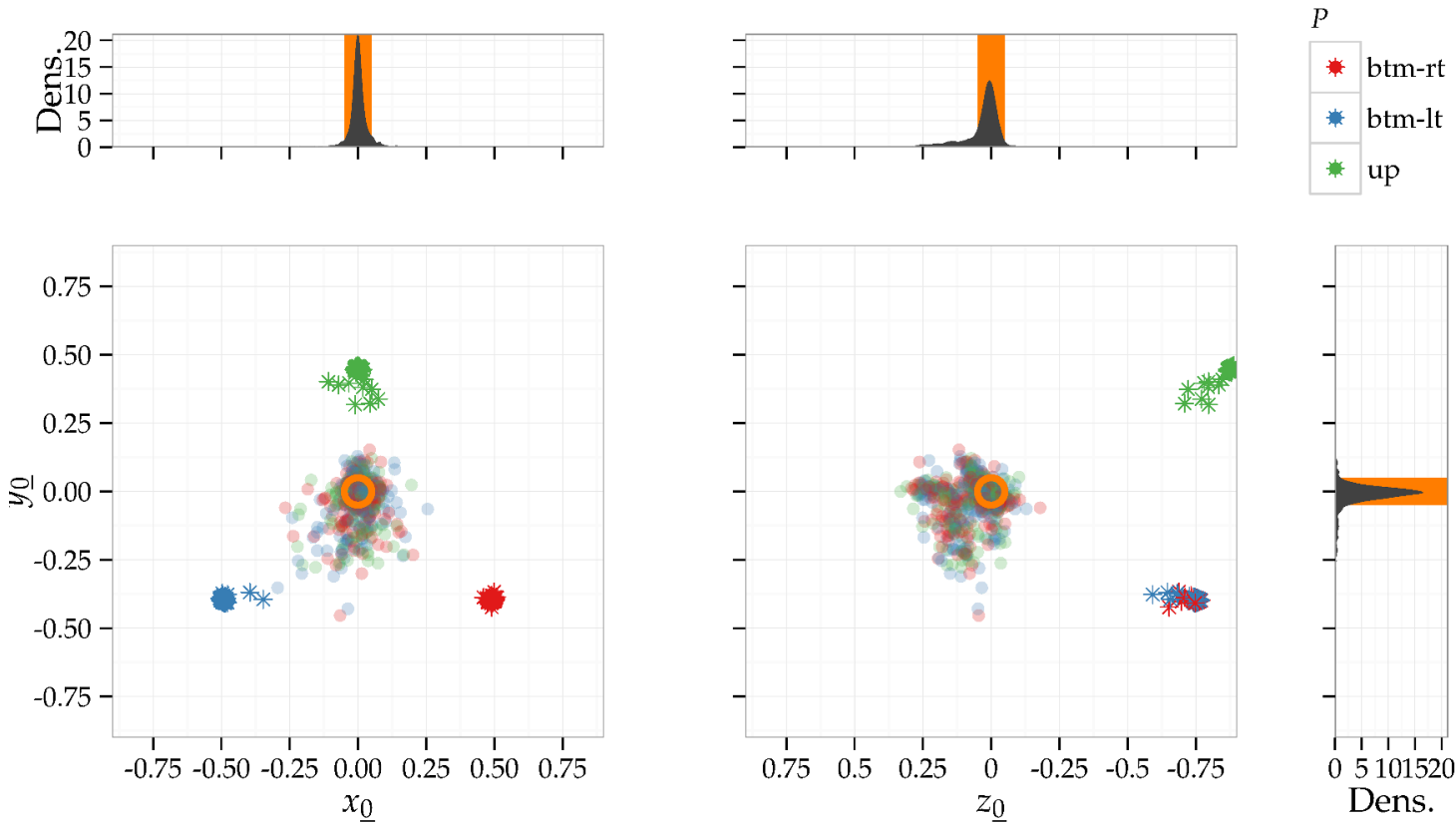

Figure 5.1 1. Front and right views of the distribution of wand positions at the frame where the goal sphere was highlighted in the 3-sphere trials. Red, blue, and green points represent the wand positions in trials where the sphere was at the bottom-right, bottom-left, and up, respectively. Correspondingly colored stars represent the sphere positions in those frames. The orange circle represents $s p h_{\underline{0}}$. The top, and side black ribbons represent the densities of the distributions of $x_{\underline{0}}$ and $z_{\underline{0}}$, and $y_{\underline{0}}$, overlayed on the length of $s p h_{\underline{0}}$ along that coordinate shown in orange. Coordinates are relative to $P_{w, \underline{0}}$. 


\subsubsection{Predictive accuracy}

At each frame $(t)$ after the goal sphere is highlighted, $\widehat{T}$ Score, and $d$ Score are calculated, with $N \in\{1,2\} \times$ decay $\in\{0.5,0.9\}$. For each of these scores, the predicted sphere corresponds to the sphere with the maximum score. Subsequently, nine frames per trial are extracted, corresponding to the last frame before $0.1 T, 0.2 T, \cdots, 0.9 T, T$, where $T$ is the time required to complete each trial. Finally, the accuracy across trial percentages $p T$ is calculated, the results are presented in Figure 5.12.

The results indicate that the $\widehat{T} S$ core predictor is better than the $d S c o r e$ predictor before $50 \%$ of the total trial time $T$ has elapsed. Before $0.5 \mathrm{~T}$, the $d S$ core predictor is less reliable than chance; after $0.5 \mathrm{~T}$, the $d$ Score predictor's accuracy gets gradually better than that of $\widehat{T}$ Score. This change in accuracy may reflect an increasing error in the $\widehat{M T}$ predictions yielded by Equation 5.5 with the parameter estimates from Table 5.1. The increased error, however, is expected given that the $D_{s}$, and $D_{m}$ predictors at $T>0$ are outside of the range of the $D_{s}$, and $D_{m}$ used to train the model, i.e., those at $T=0$. Alternatively, this change could indicate a shift to a ballistic phase in which $D \simeq D_{m}$ is most influential on $T$. Finally, notice that at $1.0 T$, none of the predictors have $100 \%$ accuracy, suggesting that the decay rates may be too low for the explored tasks.

\subsubsection{Six-sphere trials}

\subsubsection{Wand positions at the highlight frame}

As shown in Figure 5.13, the percentage of wand positions within $s p h_{0}$ at the highlight frame was very similar to the 3-sphere block, $p\left(\left\|P_{w}-P_{w, \underline{0}}\right\|<0.05\right)=0.6$. As in the 3-sphere block, participants who did not have their wand within $s p h_{\underline{0}}$ tended to place their wand closer to their bodies, $p\left(P_{w, z}-z_{\underline{0}}>0.05\right)=0.25$, but also to the right of $s p h_{\underline{0}}, p\left(P_{w, x}-x_{\underline{0}}>\right.$ $0.05)=0.13$. Given that most participants were right handed, this suggests a search for a more comfortable waiting position.

\subsubsection{Predictive accuracy}

The accuracy of $\widehat{T} S$ core, and $d$ Score across trial percentages $0.1 T, 0.2 T, \cdots, 0.9 T, T$ is calculated following the same procedure as in the 3-sphere trials. The results are presented in Figure 5.12.

The results indicate that the $\widehat{T} S$ core predictor is better than the $d S c o r e$ predictor before $40 \%$ of the total trial time $T$ has elapsed. Before $0.4 T$, the $d S c o r e$ predictor is less reliable than chance; instead, between $[0.5 \mathrm{~T}, 0.7 \mathrm{~T}]$, the $\widehat{T}$ Score predictor is less reliable than chance; and between $[0.8 T, 1.0 T]$, both predictors have fairly similar accuracy except when $N=1$, and decay $=0.9$, in which case the $d S$ core predictor is about $5 \%$ better. Notice, once again, that at $1.0 \mathrm{~T}$, none of the predictors get $100 \%$ accuracy, suggesting that the decay rates may be too low for the explored tasks. 


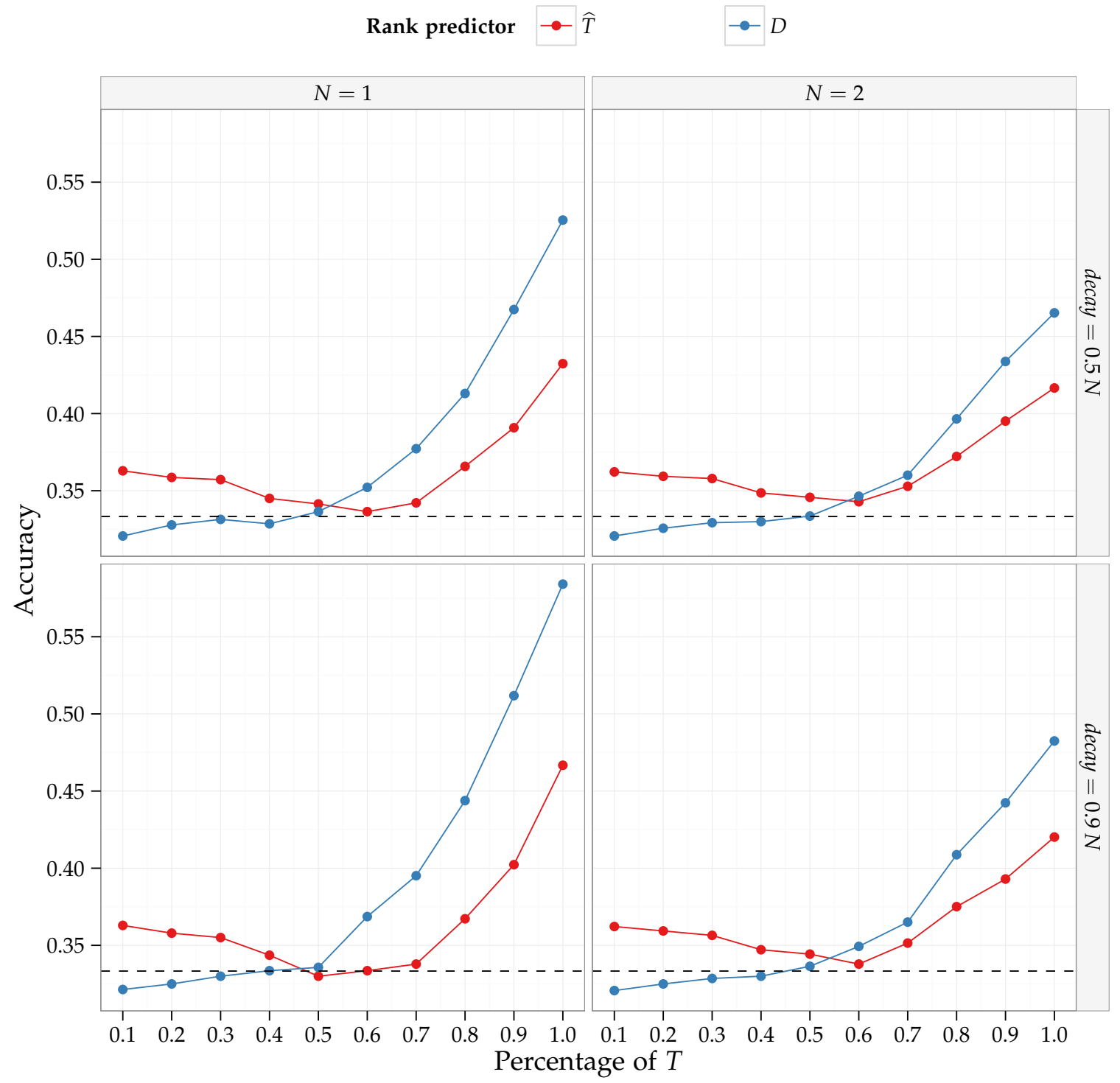

Figure 5.12. Three-sphere accuracy comparison for the $\widehat{T} S$ core, and $d S c o r e$ predictors for trial percentages $0.1 T, 0.2 T, \cdots, 0.9 T, T$, using different $N$, and decay parameters. The dotted lines represent the accuracy given by chance, i.e. $1 / 3$. 

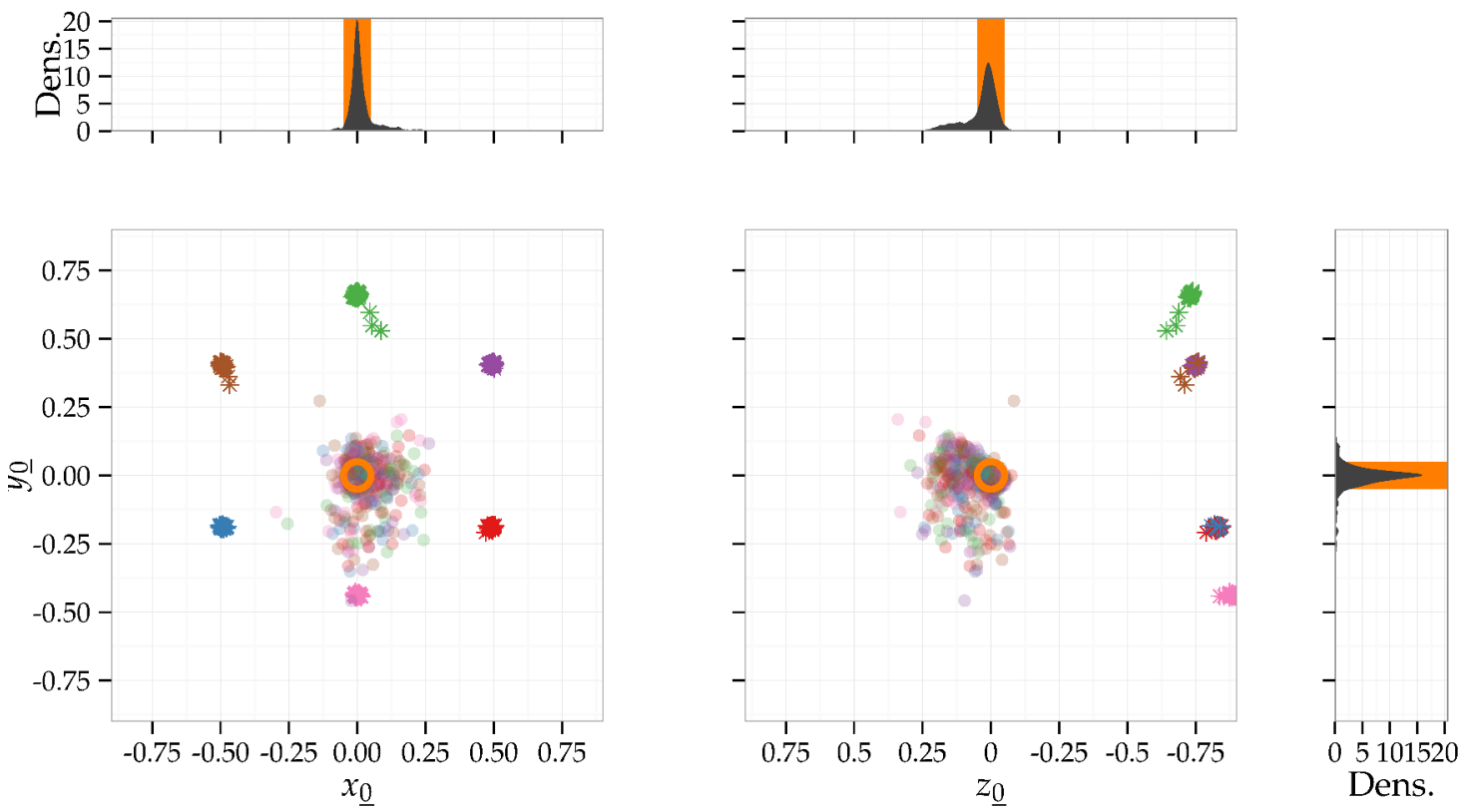

Figure 5.13. Front and right views of the distribution of wand positions at the frame where the goal sphere was highlighted in the 6-sphere trials. Red, pink, blue, brown, green, and purple points represent the wand positions in trials where the sphere was at the bottom-right, bottom, bottom-left, up-left, up, and up-right, respectively. Correspondingly colored stars represent the sphere positions in those frames. The orange circle represents $s p h_{\underline{0}}$. The top, and side black ribbons represent the densities of the distributions of $x_{\underline{0}}$ and $z_{\underline{0}}$, and $y_{\underline{0}}$, overlayed on the length of $s p h_{\underline{0}}$ along that coordinate shown in orange. Coordinates are relative to $P_{w, \underline{0}}$. Only a sample of 1,000 trials out of the 2,705 successful trials is shown. 


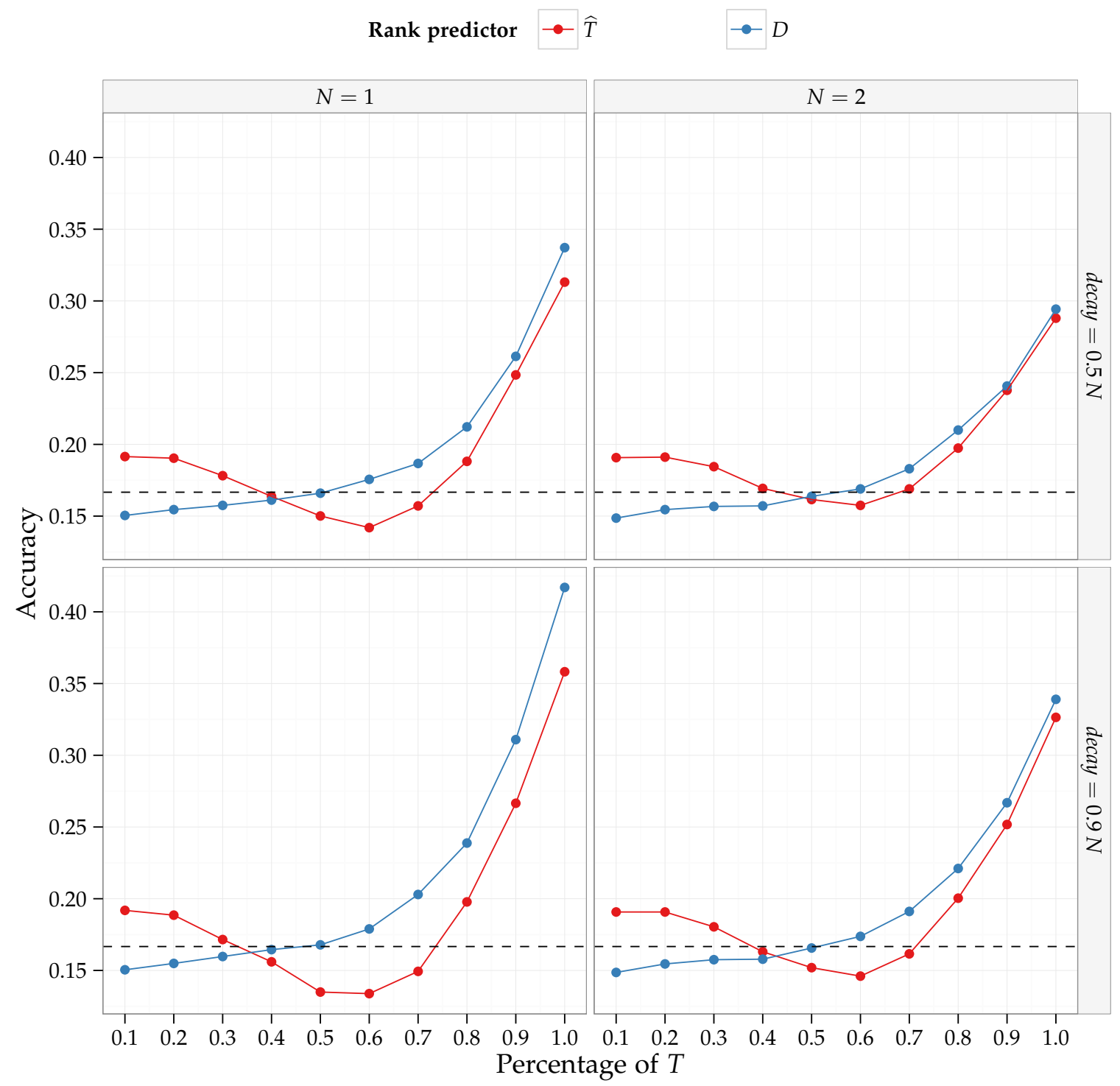

Figure 5.14. Three-sphere accuracy comparison for the $\widehat{T} S$ core, and $d S$ core predictors for trial percentages $0.1 T, 0.2 T, \cdots, 0.9 T, T$, using different $N$, and decay parameters. The dotted lines represent the accuracy given by chance, i.e. $1 / 3$. 


\subsection{Discussion}

Results are summarized according to the study hypotheses, each followed by a discussion.

\subsubsection{Moving-target selection performance in 3-D}

H1. The three-part model with per-speed coefficients, and per-velocity intercept, described in Equation (5.5), accurately models the movement time of three-dimensional moving-target selection tasks. Supported.

The three-part moving-target selection model of Equation (5.5),

$$
M T=a_{V}+b_{V} \sqrt{D_{s}}+c_{V} \sqrt{D_{m}}+d_{V} \log _{2}\left(\frac{2 D_{m}}{W}\right),
$$

yielded a good fit, with credible values of $R^{2} \in[0.89,0.96]$, presented in Table 5.1. The residuals, displayed in Figure 5.10, indicated a wider variance for $V=0.5$, compared to $V \in 1.0,1.5$, but given that there were only two visible outliers, no further modifications to the model were considered. Given that a very similar model was used to predict $P D$, these results give further evidence to the relation between $P D$, and $M T$.

H1.1. The azimuth $\alpha$, altitude $\theta$, and $\gamma$ angles, not modeled in Equation (5.5), have a small effect on MT. Partially supported.

The $\alpha$, and $\theta$ angles were varied simultaneously as sphere positions up, bottom-right, and bottom-left, which impeded the evaluation of their effects separately. Figure 5.6 suggested that targets located upward with respect to $s p h_{\underline{0}}$ had a larger MT than targets located below $s p h_{\underline{0}}$, but this effect was not visible in the residual plot (Figure 5.10).

Finally, as seen in Figure 5.7, MT remained very similar across the range of assigned $\gamma$ angles. This suggests a small effect of $\gamma$ on $M T$.

H1.1. In Equation (5.5) the coefficients $a_{V}, b_{V}, c_{V}$, and $d_{V}$ vary linearly with $V$. Supported.

The results suggest that coefficients $a_{V}, b_{V}, c_{V}$, and $d_{V}$ of Equation (5.5) decrease linearly with the increase in $V$. Using the credible intervals presented in Table 5.3 allows MT to be predicted using different $V$, while still propagating the uncertainty in these estimates.

\subsubsection{Predicting user intention in 3-D moving-target directed-selection tasks}

H2. At any given instant, the minimal movement time estimated by Equation (5.5) can be used to predict the intended target in a 3-D moving-target directed-selection task. Partially supported. 
Even though it was possible to predict the intended sphere using the estimated MT via the $\widehat{T} \operatorname{Score}_{i}(t)$ scoring function with mostly above random accuracy, the resulting accuracy was very low. In certain cases in the 6-sphere block, the accuracy was actually worse than chance, as shown in Figure 5.14.

Notice, however, that the parameter estimates used to calculate $\widehat{M T}$ were obtained from the 1-sphere block, which included a different range of $D_{s}$, and $D_{m}$ inputs, had $R T=0$, and did not have issues of target clutter or occlusion, as opposed to the 3- and 6-sphere blocks. Even though the Hick-Hyman law allows modeling $R T=\log _{2}(I+1)$, where $I$ is the number of targets in the scene, as the $R T$ elapses, the $D_{m}$ is reduced by $R T V$, and the initial wand position may change, possibly resulting in a different $D_{s}$. Therefore, integrating the Hick-Hyman law in the current model is not straightforward.

A more viable way to enhance $\widehat{M T}$ predictions in blocks with multiple targets, and presumptively the accuracy of $\widehat{T} S \operatorname{core}_{i}(t)$, would be to include subject variations in the parameters of Equation (5.5). Indeed, the parameter estimates of Table 5.1 are given for the average $M T$ per-condition, and not for all trials.

H2.1. The accuracy of the predictions based on estimated movement time will be greater than those based on wand-target distance. Partially supported.

The $\widehat{T} S \operatorname{core}_{i}(t)$ scoring function yielded better accuracies than $d \operatorname{Score}_{i}(t)$ only for trials before $0.5 \mathrm{~T}$ and $0.4 \mathrm{~T}$ in the 3- and 6-sphere blocks, respectively. In all subsequent times, $d \operatorname{Score}_{i}(t)$ was consistently more accurate. As previously suggested, this inversion in prediction accuracy may indicate a shift in movement to a ballistic phase as the trial advances, in which $D \simeq D_{m}$ is most influential on $T$.

\subsection{Conclusion}

This chapter explored the possibility of using the 2-D moving-target models for PD presented in Chapter 4 to predict performance and intention in 3-D moving-target selection. The model yielded a good fit on the $\overline{M T}$ values of the 1-sphere block, but it exhibited poor, yet above random, accuracy predicting intended targets in the 3- and 6-sphere blocks. Future work should explore possible extensions to this model to accommodate for user variations. Additionally, as shown in Chapter 3, combining this predictor with other features may result in considerable increases in accuracy.

To the author's knowledge, this is the first study to model MT in 3-D moving-target selection tasks, as well as the first study to model MT for moving-target selection tasks of any dimensionality in which the target is not moving directly toward, or directly away from the cursor. 


\section{ChAPTER 6}

\section{CONCLUSION}

The current work provides an in-depth, empirical investigation on moving-target selection, a task that is increasingly common, yet largely understudied in human-computer interaction. In particular, predictive models were developed for three of the stages involved in the action of selecting a moving target: the intended selection, the prospective difficulty selection, and the selection performance.

Intention prediction models were developed using decision trees and scoring functions with features specific to undirected selection tasks (i.e., tasks in which the users are free to select an object among multiple others), and generalizable features that can also be used in directed selection tasks (i.e., the more common experimental tasks in which users are instructed to select a specific object). As shown in Chapter 3, target size $(W)$ was deemed as the most predictive feature for intention in undirected selection, with an accuracy of $(\sim 71 \%)$ for two targets with equal velocities, but different initial positions-this was contrary to the expectation that $I D$, measured at the start of each trial, would be the most predictive feature in this type of task. In terms of generalizable features in undirected selection, results presented in Chapter 3 indicate that a combination of head-target relative gaze $(\Delta d o t)$, and cursor-target relative distance $(\Delta D)$, averaged in a 1-1.5 s time window, were predictive of selection intention with an accuracy of $(\sim 72 \%)$. The combination of these task-specific and generalizable features in undirected selection resulted in an accuracy of $(\sim 78 \%)$. Finally, in Chapter 5, scoring functions based on the predicted movement time $(\widehat{M T})$, and cursor-target distance, yielded poor, yet above random, accuracy in predicting the intended target in a directed selection task with 3 and 6 spheres.

Prospective difficulty $(P D)$ models were developed and evaluated in 1-D, and 2-D movingtarget selection tasks in Chapter 4 . These models describe the motion required to attain a target in three parts: two ballistic motions, one to align the cursor with the target's movement axis and one in-line with the target's movement axis, and a homing in motion in-line with the target's movement axis. The ballistic motions were represented with square-root distances, whereas the homing-in motion was represented with Fitts' ID. Overall, PD was shown to increase with target speed $(V), I D$, and the cursor-target-axis distance, and to decrease with 
the cursor-target distance along the target's movement axis. Contrary to the expectations, the ballistic motion required to align the cursor with the target's movement axis, and the homing-in motion along the target's movement axis were minimally affected by target speed.

Performance prediction was explored in Chapter 5 by evaluating the usage of the 2-D model of $P D$ presented in Chapter 4 as a mean movement time $(\overline{M T})$ predictor in 3-D movingtarget selection. The three-part model yielded a good fit with the observed data, but, contrary to the PD model, the coefficients related to ballistic and homing-in motions decreased as the target speed increased. Such a difference was expected given that faster targets require shorter movement times to be successfully selected.

\subsection{Limitations and future work}

\subsubsection{Intention prediction models}

Overall, the main limitation of the studied intention prediction models is that they were generated based on a post hoc analysis of moving-target selection. Nevertheless, their usefulness to address the challenges of moving-target selection needs to be assessed in an interactive context, where predictions can be integrated to enhance the moving-target task (e.g., using predictions as inputs of the enhancements described in Section 1.1.2.

Decision trees can integrate different predictive features and can be interpreted as simple ifelse rules, allowing them to be implemented in real-time. If the predictions were to be adapted during execution, however, the major difficulty would be to recalculate the trees in real-time without impacting performance. Future work should explore the possible optimizations that would allow adaptation of decision trees during interaction.

Scoring functions, on the other hand, use only one input feature, but can adapt in real-time to changes in user and target states with a small computational overhead. Unfortunately, the accuracy of such functions was very poor in directed tasks, which could indicate the limitations of using a single feature for scoring. Future work should explore accuracy enhancements to the proposed scoring functions, which could include integration of different features as composite scores, as well as parameter tuning.

\subsubsection{PD prediction models}

In order to develop the models for $P D$ in 2-D moving-target selection mentioned earlier in this chapter, the existing PD model for 1-D static-target selection was first extended to 2-D. Thus, the limitations and future work related to the formulation of this 2-D static-target model are presented prior to those related to the $1-\mathrm{D}$, and 2-D moving-target models. 


\subsubsection{Two-dimensional static-target tasks}

Target angle $\theta$. The main limitation of the 2-D static target tasks evaluated in Chapter 4 was the small range of explored target angles $\theta$. Although the literature shows that both diagonal and vertical targets are the hardest to attain, this study explored only diagonal, right-handed angles, for which the angle effect was very small. It is possible, however, that vertical targets could have a larger impact on $P D$, due to the tendency to perceive vertical distances as longer than horizontal distances [Higashiyama 1996]. To have a better understanding of the effect of $\theta$, future work on 2-D static-targets should include a wider range of target angles.

Underlying function for $a_{\theta}$. Perhaps related to the small range of explored angles, was the inability to find an underlying function for $a_{\theta}$. Neither of the functional forms of $f(\theta)$ suggested by Murata and Iwase [2001] or Appert et al. [2008] gave a reasonable approximation to $a_{\theta}$.This prevents generalization of the 2-D model, and making $P D$ predictions for other angles based on it. Yet, assuming that the target-angle effect is small for most angles $\theta \in[0,360)^{\circ}$, it may be possible to get reasonable predictions using the 1-D static-target formula.

Future work should continue to explore the underlying function for the angle effect represented in $a_{\theta}$. Once again, this exploration may be facilitated by including a wider variety of $\theta$ angles in the experimental conditions.

\subsubsection{Moving-target tasks}

Target velocity $(V, \phi)$. Similar to static-target tasks, the main limitation in the evaluated moving-target tasks was the small range of explored target speeds $V$, and movement directions $\phi .{ }^{1}$ With the current range of evaluated velocities, it is difficult to generalize the results to additional experimental conditions; even though the wide credible intervals obtained from the hierarchical models allow propagating this uncertainty to predictions on new data. Future work should include a wider variety of velocities and compare the current models' predictions to the actual observations.

Initial target angle $\theta$. In all of the evaluated moving-target pointing tasks, the initial target angle $\theta=0$. Based on the 2-D static-target model, different target angles could be represented with different intercepts, which would result in a $a_{V \times \theta}$ term. It is hypothesized, however, that the $\theta$ effect will have a minimal impact on $P D$, especially for tasks where $\phi \notin\{0,180\}^{\circ}$, since in those cases the $\theta$ angle is changing at every frame. Future work should explore these claims with experiments including initial target angles $\theta \in[0,360)^{\circ}$.

Inter-formula compatibility. The 1-D moving-target pointing model is not nested within the 2-D model, i.e., there is no constraint on the 2-D model that would lead to the 1-D one. Specifically, the ID term varies per speed $V$ for the 1-D moving-target formula, but it stays

\footnotetext{
${ }^{1}$ It is worth noting that previous experiments on moving-target selection included only two [Jagacinski et al. 1980] or three [Al Hajri et al. 2011] levels of non-zero speeds, and none of them explored target movement directions $\phi \notin\{0,180\}^{\circ}$.
} 
almost constant in the 2-D formula. This may imply that there is a shortcoming in the derivation of the 2-D formula, or that 1-D moving-target tasks are inherently different from 2-D ones (e.g., because users may be more inclined to wait for the target in the former), and this difference is reflected in an interaction between ID and $V$ that does not happen in 2-D.

Nevertheless, the six bottom panes of Figure 4.13 indicate that the 1-D moving-target data can be adequately described by the 2-D moving-target model. Once again, future work should evaluate these formulae with an extended range of velocities to evaluate this apparent incongruence and explore the theoretical and practical limitations of the proposed models.

\subsubsection{Methods for assessing PD}

A possible shortcoming in the evaluation of the proposed PD models concerns the reliability of the prospective assessments given by the user on each task. By using a discrete scale to evaluate a continuous quantity, there is an inherent loss of precision in each assessment. Regardless of the scale, it may be difficult for participants to quantify their prospective judgments. Therefore, future work should also attempt to assess the proposed models of $P D$ in moving-target selection using measures that do not rely on self assessment, such an approach has been explored in static-target selection using EEG [Kourtis et al. 2012].

\subsubsection{Performance prediction models}

\subsubsection{Target velocity}

Similar to $P D$, the study of $M T$ for tasks with a wider range of $V$, and $\phi$ values would allow further generalization of the proposed models. In particular, more precise estimates for the linear relation between $V$ and the coefficients of Equation (5.5) can be obtained by studying selection tasks with additional levels of $V$.

\subsubsection{Target distance}

In the design of the experiment described in Chapter 5, D was kept constant at $1 \mathrm{~m}$, and the change in $D_{m}$, and $D_{s}$, was ensured by changing the $\phi$ angle. This constraint allowed the study of other experimental conditions without making the main study too long, but led to heavily correlated $D_{m}$, and $D_{s}$ values. Even though the variability in initial wand positions mitigated this effect, such a correlation impedes a clear distinction of the effects of $D_{m}$, and $D_{s}$ on $M T$. Therefore, future work should explore different $D$ values.

\subsubsection{Additional measures of performance}

In accordance with the main body of target selection in HCI, the analysis of performance was mostly limited to MT. However, considering that there is a critical velocity beyond which selection is impossible, it seems important to quantify the effect of $V$, and other experimental factors, on target success rate $(\rho)$. Predicting $\rho$ would allow a reevaluation of 
the Hoffmann [1991] moving-target model, ${ }^{2}$ as well as the refinement of intention prediction models, which are currently limited to successful trials. Thus, future work on moving-target selection should also evaluate and attempt to model $\rho$, and other measures of performance.

\subsubsection{General issues}

\subsubsection{Target shape}

Target shape was not considered in the development of any of the models. Nevertheless, a formulation for 2-D bivariate targets (e.g., rectangles, where $W \neq H$ ) was suggested for $P D$, in which each $\theta \times(W / H)$ combination should have its own intercept, as specified in Equation (4.10). The major drawback of this solution is that the degrees of freedom of the model are reduced with each $\theta \times(W / H)$ combination, requiring a large number of data points to be evaluated. This approach can also be generalized to 3-D.

For more complex shapes a different ID term, such as those proposed in [Sheikh and Hoffmann 1994; Murata 1999; Grossman and Balakrishnan 2005], may be necessary. Since these ID terms implicitly include the target's angular position effect, the per-angle intercept may add unnecessary redundancy to the model. Therefore, future work should explore the effect of target shape on $P D$ and $M T$.

\subsubsection{Target acceleration}

In the explored moving-target selection tasks, the target velocity was always constant. Such simplification is acceptable in certain tasks where targets have nearly constant velocities (e.g., in air traffic control systems, or in tasks where objects are moving on a conveyor belt) but may not be reasonable for most motions that follow real-world dynamics. Thus, it is likely that the proposed models for $P D$, and $M T$ will yield less accurate responses for targets whose velocities are markedly non-constant. On the other hand, intention predictions based on inputs measured throughout a time window are less likely to suffer from a loss in accuracy due to changes in velocity. ${ }^{3}$ To evaluate these claims, future work should investigate the effect of target acceleration on selection performance, prospective difficulty, and intention detection.

\footnotetext{
${ }^{2}$ As described in Chapter 2, the usage of the Hoffmann moving-target model without $\rho$ results in potentially harder and unreliable calculations.

${ }^{3}$ Indeed, in Ortega's experiment [Ortega 2013], target movement directions were constantly changing.
} 


\section{BIBLIOGRAPHY}

Johnny Accot and Shumin Zhai. 2003. Refining Fitts' Law Models for Bivariate Pointing. In Proceedings of the SIGCHI Conference on Human Factors in Computing Systems (CHI '03). ACM, Ft. Lauderdale, Florida, USA, 193-200. DoI : http:/ / dx.doi.org/10.1145/642611.642646

Abir Al Hajri, Sidney Fels, Gregor Miller, and Michael Ilich. 2011. Moving Target Selection in 2D Graphical User Interfaces. In Proceedings of the 13th IFIP TC 13 International Conference on Human-Computer Interaction - Volume Part II (INTERACT '11), Pedro Campos, Nicholas Graham, Joaquim Jorge, Nuno Nunes, Philippe Palanque, and Marco Winckler (Eds.). SpringerVerlag, Lisbon, Portugal, 141-161. Dor :http:/ / dx.doi.org/10.1007/978-3-642-23771-3_12

Caroline Appert, Olivier Chapuis, and Michel Beaudouin-Lafon. 2008. Evaluation of Pointing Performance on Screen Edges. In Proceedings of the Working Conference on Advanced Visual Interfaces (AVI '08). ACM, Napoli, Italy, 119-126. DoI: http:/ / dx.doi.org/10.1145/1385569.1385590

Ferran Argelaguet and Carlos Andujar. 2013. A survey of 3D object selection techniques for virtual environments. Computers $\mathcal{E}$ Graphics 37, 3 (2013), 121-136. DoI : http:/ / dx.doi.org/10.1016/j.cag.2012.12.003

Jeremy N. Bailenson, Michael S. Shum, and David H. Uttal. 2000. The initial segment strategy: a heuristic for route selection. Memory $\mathcal{E}$ Cognition 28, 2 (March 2000), 306-18. DoI : http: / / dx.doi.org/10.3758/BF03213808

Christopher M. Bishop. 2006. Pattern Recognition and Machine Learning (9 ed.). Springer, New York, New York, USA. 738 pages.

Renaud Blanch and Michaël Ortega. 2009. Rake cursor: improving pointing performance with concurrent input channels. In Proceedings of the SIGCHI Conference on Human Factors in Computing Systems (CHI '09). ACM, Boston, Massachusetts, USA, 1415-1418. DoI : http:/ / dx.doi.org/10.1145/1518701.1518914

James Boritz, Kellogg S. Booth, and William B. Cowan. 1991. Fitts's Law Studies of Directional Mouse Movement. In Graphics Interface'91 (GI '91). Canadian Information Processing Society, Calgary, Alberta, Canada, 216-223. 
Doug A. Bowman, Ernst Kruijff, Joseph J. LaViola, and Ivan Poupyrev. 2004. 3D User Interfaces: Theory and Practice. Addison-Wesley Professional, Boston, Massachusetts, USA. 512 pages.

George E. P. Box and Norman R. Draper. 1987. Empirical Model-Building and Response Surfaces. John Wiley \& Sons, Inc., New York, New York, USA. 669 pages.

Stuart K. Card, William K. English, and Betty J. Burr. 1978. Evaluation of Mouse, RateControlled Isometric Joystick, Step Keys, and Text Keys for Text Selection on a CRT. Ergonomics 21, 8 (1978), 601-613. Dor : http:/ / dx.doi.org/10.1080/00140137808931762

Stuart K. Card, Thomas P. Moran, and Allen Newell. 1980. The Keystroke-Level Model for User Performance Time with Interacive Systems. Commun. ACM 23, 7 (July 1980), 396-410. Dor :http:/ / dx.doi.org/10.1145/358886.358895

Stuart K. Card, Thomas P. Moran, and Allen Newell. 1983. The Psychology of Human-Computer Interaction. Vol. 97. Lawrence Erlbaum Associates, Publishers, Hillsdale, New Jersey. 625 pages. DoI:http://dx.doi.org/10.2307/1422176

Heather Carnahan and Bradford J. McFadyen. 1996. Visuomotor control when reaching toward and grasping moving targets. Acta Psychologica 92, 1 (1996), 17-32. DoI : http:/ / dx.doi.org/10.1016/0001-6918(95)00006-2

Juan Sebastián Casallas, James H. Oliver, Jonathan W. Kelly, Frédéric Merienne, and Samir Garbaya. 2013. Towards a Model for Predicting Intention in 3D Movingtarget Selection Tasks. In Proceedings of the 10th International Conference on Engineering Psychology and Cognitive Ergonomics: Understanding Human Cognition - Volume Part I (EPCE '13), Don Harris (Ed.). Springer-Verlag, Las Vegas, Nevada, USA, 13-22. DoI : http:/ / dx.doi.org/10.1007/978-3-642-39360-0_2

Juan Sebastián Casallas, James H. Oliver, Jonathan W. Kelly, Frédéric Merienne, and Samir Garbaya. 2014. Using Relative Head and Hand-Target Features to Predict Intention in 3D Moving-Target Selection. In Proceedings of the 2014 IEEE Virtual Reality Conference (VR 2014). IEEE, Minneapolis, Minnesota, USA, 51-56. Dor :http:/ / dx.doi.org/10.1109/VR.2014.6802050

Alan H. S. Chan and Errol R. Hoffmann. 2013. Subjective Difficulty of Movements With Ongoing Visual Control. Journal of Motor Behavior 45, 6 (2013), 507-517. DoI : http:/ / dx.doi.org/10.1080/00222895.2013.833081

Nicholas Christenfeld. 1995. Choices from Identical Options. Psychological Science 6, 1 (1995), 50-55. Dor: http:/ / dx.doi.org/10.1111/j.1467-9280.1995.tb00304.x 
Sue V. G. Cobb, Sarah Nichols, Amanda Ramsey, and John R. Wilson. 1999. Virtual RealityInduced Symptoms and Effects (VRISE). Presence: Teleoperators E Virtual Environments 8, 2 (April 1999), 169-186. DoI: http://dx.doi.org/10.1162/105474699566152

Gerwin de Haan, Michal Koutek, and Frits H. Post. 2005. IntenSelect: using dynamic object rating for assisting 3D object selection. In Proceedings of the 11th Eurographics conference on Virtual Environments (EGVE'05). Eurographics Association, Aalborg, Denmark, 201-209. DoI : http:/ / dx.doi.org/10.2312/EGVE/IPT_EGVE2005/201-209

Didier Delignières. 1990. La difficulté en escalade : exigences objectives et perception des exigences des tâches motrices. Diplôme de l'I.N.S.E.P. Institut national du sport et de l'éducation physique.

Didier Delignières. 1993. Approche psychophysique de la perception de la difficulté dans les tâches perceptivo-motrices. Ph.D. Dissertation. Université Paris V.

Didier Delignières and Jean-Pierre Famose. 1992. Perception de la difficulté, entropie et performance. Science $\mathcal{E}$ Sports 7, 4 (1992), 245-252. DoI : http:/ / dx.doi.org/10.1016/S0765-1597(05)80097-8

Pierre Dragicevic, Fanny Chevalier, and Stephane Huot. 2014. Running an HCI Experiment in Multiple Parallel Universes. In CHI '14 Extended Abstracts on Human Factors in Computing Systems (CHI EA '14). ACM, Paris, France, 607-618. DoI : http: / / dx.doi.org/10.1145/2559206.2578881

Heiko Drewes. 2010. Only One Fitts' Law Formula Please!. In CHI '10 Extended Abstracts on Human Factors in Computing Systems (CHI EA '10). ACM, Atlanta, Georgia, USA, 2813-2822. DoI : http:/ / dx.doi.org/10.1145/1753846.1753867

J.P. Famose, D. Delignières, and J. Genty. 1991. Perception de la difficulté dans une tâche d'anticipation-coïncidence. In IVèmes Journées Internationales d'Automne de L'A.C.A.P.S. Lille, France, $1-4$.

Paul M. Fitts. 1954. The Information Capacity of the Human Motor System in Controlling the Amplitude of Movement. Journal of Experimental Psychology 47, 6 (1954), 381-391. Dor : http:/ / dx.doi.org/10.1037/h0055392

Jérémie Francone and Laurence Nigay. 2011. Using the user's point of view for interaction on mobile devices. In 23rd French Speaking Conference on Human-Computer Interaction (IHM '11). ACM, Sophia Antipolis, France, 4:1-4:8. DoI : http://dx.doi.org/10.1145/2044354.2044360

Khai-Chung Gan and Errol R. Hoffmann. 1988. Geometrical conditions for ballistic and visually controlled movements. Ergonomics 31, 5 (1988), 829-839. DoI : http:/ / dx.doi.org/10.1080/00140138808966724 
Andrew Gelman. 2006. Prior distributions for variance parameters in hierarchical models (Comment on Article by Browne and Draper). Bayesian Analysis 1, 3 (2006), 515-534. Dor:http:/ / dx.doi.org/10.1214/06-BA117A

Andrew Gelman, John B. Carlin, Hal S. Stern, David B. Dunson, Aki Vehtari, and Donald B. Rubin. 2013. Bayesian Data Analysis (3 ed.). CRC press, Boca Raton, Florida, USA. 675 pages.

Andrew Gelman and Jennifer Hill. 2007. Data Analysis Using Regression and Multilevel/Hierarchical Models (1 ed.). Cambridge University Press, New York, New York, USA. 648 pages.

Suzanne M. Grilli. 2011. Perceived Difficulty in a Fitts Task. Master's Thesis. Cleveland State University. http://rave.ohiolink.edu/etdc/view?acc_num=csu1322544972

Tovi Grossman and Ravin Balakrishnan. 2004. Pointing at Trivariate Targets in 3D Environments. In Proceedings of the SIGCHI Conference on Human Factors in Computing Systems (CHI '04). ACM, Vienna, Austria, 447-454. Dor : http://dx.doi.org/10.1145/985692.985749

Tovi Grossman and Ravin Balakrishnan. 2005. A Probabilistic Approach to Modeling Twodimensional Pointing. ACM Transactions on Computer-Human Interaction 12, 3 (Sept. 2005), 435-459. Dor: http:/ / dx.doi.org/10.1145/1096737.1096741

Yves Guiard and Michel Beaudouin-Lafon. 2004. Fitts' law 50 years later: applications and contributions from human-computer interaction. International Journal of Human-Computer Studies 61, 6 (Dec. 2004), 747-750. Dor : http:/ / dx.doi.org/10.1016/j.ijhcs.2004.09.003

Tyler J Gunn, Pourang Irani, and John Anderson. 2009. An evaluation of techniques for selecting moving targets. In CHI '09 Extended Abstracts on Human Factors in Computing Systems (CHI EA '09). ACM, New York, NY, USA, 3329-3334. Dor :http:/ / dx.doi.org/10.1145/1520340.1520481

Mark Hall, Hazeltine National, Eibe Frank, Geoffrey Holmes, Bernhard Pfahringer, Peter Reutemann, and Ian H. Witten. 2009. The WEKA Data Mining Software : An Update. SIGKDD Explorations Newsletter 11, 1 (2009), 10-18. DoI : http:/ / dx.doi.org/10.1145/1656274.1656278

Mark S. Hancock and Kellogg S. Booth. 2004. Improving Menu Placement Strategies for Pen Input. In Proceedings of Graphics Interface 2004 (GI '04). Canadian Human-Computer Communications Society, London, Ontario, Canada, 221-230. http://dl.acm.org/citation. cfm?id=1006058.1006085 
Khalad Hasan, Tovi Grossman, and Pourang Irani. 2011. Comet and Target Ghost: Techniques for Selecting Moving Targets. In Proceedings of the SIGCHI Conference on Human Factors in Computing Systems (CHI '11). ACM, Vancouver, British Columbia, Canada, 839-848. DoI: http:/ / dx.doi.org/10.1145/1978942.1979065

Mary Hegarty and David Waller. 2004. A dissociation between mental rotation and perspective-taking spatial abilities. Intelligence 32, 2 (2004), 175-191. DoI : http:/ / dx.doi.org/10.1016/j.intell.2003.12.001

William Edmund Hick. 1952. On the rate of gain of information. Quarterly Journal of Experimental Psychology 4, 1 (1952), 11-26. DoI : http:/ / dx.doi.org/10.1080/17470215208416600

Atsuki Higashiyama. 1996. Horizontal and vertical distance perception: The discorded-orientation theory. Perception $\mathcal{E}$ Psychophysics 58, 2 (1996), 259-270. DoI : http:/ / dx.doi.org/10.3758/BF03211879

Errol R. Hoffmann. 1991. Capture of moving targets: a modification of Fitts' Law. Ergonomics 34, 2 (1991), 211-220. Dor : http://dx.doi.org/10.1080/00140139108967307

Errol R. Hoffmann. 2013. Which Version/Variation of Fitts' Law? A Critique of Information-Theory Models. Journal of Motor Behavior 45, 3 (2013), 205-215. DoI : http:/ / dx.doi.org/10.1080/00222895.2013.778815

Errol R. Hoffmann. 2014. Validity of the Shannon form of Fitts' law: a response to MacKenzie (2013). Technical Report. 22 pages. erroldot@tpg.com.au

Errol R. Hoffmann and Alan H. S. Chan. 2012. Underwater movement times with ongoing visual control. Ergonomics 55, 12 (2012), 1513-1523. Dor : http:/ / dx.doi.org/10.1080/00140139.2012.719038

Errol R. Hoffmann and Ilyas H. Sheikh. 1994. Effect of varying target height in a Fitts' movement task. Ergonomics 37, 6 (1994), 1071-1088. Dor : http:/ / dx.doi.org/10.1080/00140139408963719

Christian Holz and Patrick Baudisch. 2010. The Generalized Perceived Input Point Model and How to Double Touch Accuracy by Extracting Fingerprints. In Proceedings of the SIGCHI Conference on Human Factors in Computing Systems (CHI '10). ACM, Atlanta, Georgia, USA, 581-590. Dor: http://dx.doi.org/10.1145/1753326.1753413

Ray Hyman. 1953. Stimulus information as a determinant of reaction time. Journal of Experimental Psychology 45, 3 (March 1953), 188-196. Dor: http://dx.doi.org/10.1037/h0056940

Michael Victor Ilich. 2009. Moving Target Selection in Interactive Video. Master's Thesis. The University of British Columbia. http:/ /hdl.handle.net/2429/17444 
Richard J. Jagacinski and Donald L. Monk. 1985. Fitts' Law in Two Dimensions with Hand and Head Movements. Journal of Motor Behavior 17, 1 (1985), 77-95. DoI : http:/ / dx.doi.org/10.1080/00222895.1985.10735338

Richard J. Jagacinski, Daniel W. Repperger, Sharon L. Ward, and Martin S. Moran. 1980. A Test of Fitts' Law with Moving Targets. Human Factors: The Journal of the Human Factors and Ergonomics Society 22, 2 (1980), 225-233. Dor : http:/ / dx.doi.org/10.1177/001872088002200211

Roland S. Johansson, Göran Westling, Anders Bäckström, and J. Randall Flanagan. 2001. Eye-hand Coordination in Object Manipulation. The Journal of Neuroscience 21, 17 (Sept. 2001), 6917-32. http://www.ncbi.nlm.nih.gov/pubmed/11517279

Helmut Jorke, Arnold Simon, and Markus Fritz. 2008. Advanced Stereo Projection Using Interference Filters. In 3DTV Conference: The True Vision - Capture, Transmission and Display of 3D Video, 2008 (3DTV 2008). IEEE, Istanbul, Turkey, 177-180. DoI : http:/ / dx.doi.org/10.1109/3DTV.2008.4547837

Marcel Adam Just and Patricia A. Carpenter. 1976. Eye fixations and cognitive processes. Cognitive Psychology 8, 4 (Oct. 1976), 441-480. Dor : http:/ / dx.doi.org/10.1016/0010-0285(76)90015-3

Maurits Kaptein and Judy Robertson. 2012. Rethinking Statistical Analysis Methods for CHI. In Proceedings of the SIGCHI Conference on Human Factors in Computing Systems (CHI '12). ACM, Austin, Texas, USA, 1105-1114. Dor :http:/ / dx.doi.org/10.1145/2207676.2208557

Robert S. Kennedy, Norman E. Lane, Kevin S. Berbaum, and Michael G. Lilienthal. 1993. Simulator Sickness Questionnaire: An Enhanced Method for Quantifying Simulator Sickness. The International Journal of Aviation Psychology 3, 3 (1993), 203-220. DoI : http:/ / dx.doi.org/10.1207/s15327108ijap0303_3

Regis Kopper, Doug A. Bowman, Mara G. Silva, and Ryan P. McMahan. 2010. A human motor behavior model for distal pointing tasks. International Journal of Human-Computer Studies 68, 10 (Oct. 2010), 603-615. Dor :http:/ / dx.doi.org/10.1016/j.ijhcs.2010.05.001

D. Kourtis, N. Sebanz, and G. Knoblich. 2012. EEG correlates of Fitts's law during preparation for action. Psychological Research 76, 4 (July 2012), 514-24. Dor : http:/ / dx.doi.org/10.1007/s00426-012-0418-z

John K. Kruschke, Herman Aguinis, and Harry Joo. 2012. The Time Has Come: Bayesian Methods for Data Analysis in the Organizational Sciences. Organizational Research Methods 15, 4 (2012), 722-752. Dor : http:/ / dx.doi.org/10.1177/1094428112457829 
Edward Lank, Yi-Chun Nikko Cheng, and Jaime Ruiz. 2007. Endpoint Prediction Using Motion Kinematics. In Proceedings of the SIGCHI Conference on Human Factors in Computing Systems (CHI '07). ACM, San Jose, California, USA, 637-646. DoI: http:/ / dx.doi.org/10.1145/1240624.1240724

I. Scott MacKenzie. 1989. A Note on the Information-Theoretic Basis for Fitts' Law. Journal of Motor Behavior 21, 3 (1989), 323-330. Dor : http:/ / dx.doi.org/10.1080/00222895.1989.10735486

I. Scott MacKenzie. 1992. Fitts' Law as a Research and Design Tool in HumanComputer Interaction. Human-Computer Interaction 7, 1 (March 1992), 91-139. Dor : http:/ /dx.doi.org/10.1207/s15327051hci0701_3

Ian Scott MacKenzie. 2013. A Note on the Validity of the Shannon Formulation for Fitts' Index of Difficulty. Open Journal of Applied Sciences 3, 6 (2013), 360-368. DoI: http:/ / dx.doi.org/10.4236/ojapps.2013.36046

I. Scott MacKenzie and William Buxton. 1992. Extending Fitts' Law to Two-dimensional Tasks. In Proceedings of the SIGCHI Conference on Human Factors in Computing Systems (CHI '92). ACM, Monterey, California, USA, 219-226. Dor : http://dx.doi.org/10.1145/142750.142794

Regan L Mandryk and Calvin Lough. 2011. The Effects of Intended Use on Target Acquisition. In Proceedings of the SIGCHI Conference on Human Factors in Computing Systems (CHI '11). ACM, Vancouver, British Columbia, Canada, 1649-1652. DoI: http:/ / dx.doi.org/10.1145/1978942.1979182

Michael J. McGuffin and Ravin Balakrishnan. 2005. Fitts' law and expanding targets: Experimental studies and designs for user interfaces. ACM Transactions on Computer-Human Interaction 12, 4 (Dec. 2005), 388-422. Dor : http:/ / dx.doi.org/10.1145/1121112.1121115

Tom M. Mitchell. 1997. Machine learning (1 ed.). McGraw-Hill, Boston, MA. 432 pages.

David Mould and Carl Gutwin. 2004. The effects of feedback on targeting with multiple moving targets. In Proceedings of Graphics Interface 2004 (GI '04). Canadian Human-Computer Communications Society, School of Computer Science, University of Waterloo, Waterloo, Ontario, Canada, 25-32. http://dl.acm.org/citation.cfm?id=1006058.1006062

Atsuo Murata. 1999. Extending Effective Target Width in Fitts' Law to a Two-Dimensional Pointing Task. International Journal of Human-Computer Interaction 11, 2 (1999), 137-152. Dor: http:/ / dx.doi.org/10.1207/S153275901102_4

Atsuo Murata and Hirokazu Iwase. 2001. Extending fitts' law to a threedimensional pointing task. Human Movement Science 20, 6 (2001), 791-805. DoI : http:/ / dx.doi.org/10.1016/S0167-9457(01)00058-6 
Norman Murray, Dave Roberts, Anthony Steed, Paul Sharkey, Paul Dickerson, and John Rae. 2007. An assessment of eye-gaze potential within immersive virtual environments. ACM Transactions on Multimedia Computing, Communications and Applications 3, 4 (Dec. 2007), 8:1-8:17. Dor:http://dx.doi.org/10.1145/1314303.1314311

Mathieu Nancel, Olivier Chapuis, Emmanuel Pietriga, Xing-Dong Yang, Pourang P Irani, and Michel Beaudouin-Lafon. 2013. High-precision pointing on large wall displays using small handheld devices. In Proceedings of the SIGCHI Conference on Human Factors in Computing Systems (CHI '13). ACM, Paris, France, 831-840. DoI: http:/ / dx.doi.org/10.1145/2470654.2470773

Kai Nickel and Rainer Stiefelhagen. 2003. Pointing gesture recognition based on 3D-tracking of face, hands and head orientation. In Proceedings of the 5th International Conference on Multimodal Interfaces (ICMI '03). ACM, Vancouver, British Columbia, Canada, 140-146. DoI : http:/ / dx.doi.org/10.1145/958432.958460

Karin Nieuwenhuizen, Jean-Bernard Martens, Lei Liu, and Robert Van Liere. 2009. Insights from Dividing 3D Goal-Directed Movements into Meaningful Phases. IEEE Computer Graphics and Applications 29, 6 (Nov. 2009), 44-53. Dor :http://dx.doi.org/10.1109/MCG.2009.121

Donald A. Norman. 1986. Cognitive Engineering. In User Centered System Design: New Perspectives on Human-Computer Interaction, Donald A. Norman and Stephen W. Draper (Eds.). Lawrence Erlbaum Associates, Hillsdale, New Jersey, USA, Chapter 3, 31-61.

Donald A. Norman. 2002. The Design of Everyday Things. Basic Books, New York, New York, USA. 272 pages.

David Noy. 2001. Predicting User Intentions in Graphical User Interfaces Using Implicit Disambiguation. In CHI '01 Extended Abstracts on Human Factors in Computing Systems (CHI EA '01). ACM, Seattle, Washington, USA, 455-456. Dor : http:/ / dx.doi.org/10.1145/634067.634330

Scott Olitsky, Henry Lee, and Edward Young. 2006. IVAC_-Interactive Visual Acuity Chart. (2006). http:/ / www.smbs.buffalo.edu/oph/ped/IVAC/IVAC.html

Michaël Ortega. 2013. Hook: Heuristics for Selecting 3D Moving Objects in Dense Target Environments. In Proceedings of the IEEE 8th Symposium on 3D User Interfaces (3DUI 2013). IEEE, Orlando, Florida, USA, 119-122. DoI : http:/ / dx.doi.org/10.1109/3DUI.2013.6550208

Ryan A. Pavlik and Judy M. Vance. 2012. VR JuggLua: A framework for VR applications combining Lua, OpenSceneGraph, and VR Juggler. In 2012 5th Workshop on Software Engineering and Architectures for Realtime Interactive Systems (SEARIS). IEEE, Singapore, 29-35. DoI : http:/ / dx.doi.org/10.1109/SEARIS.2012.6231166 
Andriy Pavlovych and Carl Gutwin. 2012. Assessing Target Acquisition and Tracking Performance for Complex Moving Targets in the Presence of Latency and Jitter. In Proceedings of Graphics Interface 2012 (GI' 12). Canadian Information Processing Society, Toronto, Ontario, Canada, 109-116. http: / / dl.acm.org / citation.cfm?id=2305276.2305295

James G. Phillips and Thomas J. Triggs. 2001. Characteristics of cursor trajectories controlled by the computer mouse. Ergonomics 44, 5 (2001), 527-536. Dor : http:/ / dx.doi.org/10.1080/00140130121560

Sébastien Pierard, Vincent Pierlot, Antoine Lejeune, and Marc Van Droogenbroeck. 2012. I-see-3D! An Interactive and Immersive System that dynamically adapts 2D projections to the location of a user's eyes. In International Conference on 3D Imaging (IC3D). Liège, Belgium. http:/ / orbi.ulg.ac.be/handle/2268/134431

Ivan Poupyrev, Suzanne Weghorst, Mark Billinghurst, and Tadao Ichikawa. 1997. A framework and testbed for studying manipulation techniques for immersive VR. In Proceedings of the ACM Symposium on Virtual Reality Software and Technology (VRST '97). ACM, Lausanne, Switzerland, 21-28. DoI: http://dx.doi.org/10.1145/261135.261141

J. R. Quinlan. 1986. Induction of Decision Trees. Machine Learning 1, 1 (1986), 81-106. DoI :http:/ / dx.doi.org/10.1023/A:1022643204877

John Ross Quinlan. 1993. C4.5: Programs for Machine Learning. Morgan Kaufmann, San Mateo, California, USA. 302 pages. http://books.google.com/books?hl=en\&lr=\&id= HExncpjbYroC\&pgis=1

R Core Team. 2014. R: A Language and Environment for Statistical Computing. R Foundation for Statistical Computing, Vienna, Austria. http:/ / www.r-project.org/

R Core Team. 2015. R: A Language and Environment for Statistical Computing. R Foundation for Statistical Computing, Vienna, Austria. http:/ / www.r-project.org/

Martin Reddy. 1994. Reducing lags in virtual reality systems using motion-sensitive level of detail. In Proceedings of the 2nd UK VR-SIG Conference, Robin Hollands (Ed.). Theale, Berkshire, UK, 25-31. http: / / citeseerx.ist.psu.edu/viewdoc/download?doi=10.1.1.50.15\& rep $=$ rep $1 \&$ type $=$ pdf\#page $=27$

Jaime Ruiz and Edward Lank. 2014. Analyzing Intended Use Effects in Target Acquisition. In Proceedings of the 2014 International Working Conference on Advanced Visual Interfaces (AVI '14). ACM, Como, Italy, 145-152. DoI : http:/ / dx.doi.org/10.1145/2598153.2598158

Reza Shadmehr, Maurice A. Smith, and John W. Krakauer. 2010. Error correction, sensory prediction, and adaptation in motor control. Annual Reviews of Neuroscience 33, 1 (2010), 89-108. DoI: http:/ / dx.doi.org/10.1146/annurev-neuro-060909-153135 
Ilyas H. Sheikh and Errol R. Hoffmann. 1994. Effect of target shape on movement time in a Fitts task. Ergonomics 37, 9 (1994), 1533-1547. Dor : http:/ / dx.doi.org/10.1080/00140139408964932

Garth Shoemaker, Takayuki Tsukitani, Yoshifumi Kitamura, and Kellogg S. Booth. 2012. Two-Part Models Capture the Impact of Gain on Pointing Performance. ACM Transactions on Computer-Human Interaction 19, 4 (Dec. 2012), 28:1-28:34. DoI: http:/ / dx.doi.org/10.1145/2395131.2395135

João Silva, Diogo Cabral, Carla Fernandes, and Nuno Correia. 2012. Real-time Annotation of Video Objects on Tablet Computers. In Proceedings of the 11th International Conference on Mobile and Ubiquitous Multimedia (MUM '12). ACM, Ulm, Germany, 19:1-19:9. DoI: http:/ / dx.doi.org/10.1145/2406367.2406391

Andrew B. Slifkin and Suzanne M. Grilli. 2006. Aiming for the future: prospective action difficulty, prescribed difficulty, and Fitts' law. Experimental Brain Research 174, 4 (Oct. 2006), 746-753. DoI: http:/ / dx.doi.org/10.1007/s00221-006-0518-3

Dan Song, Nikolaos Kyriazis, Iason Oikonomidis, Chavdar Papazov, Antonis Argyros, Darius Burschka, and Danica Kragic. 2013. Predicting human intention in visual observations of hand/object interactions. In Proceedings of the 2013 IEEE International Conference on Robotics and Automation (ICRA 2013). IEEE, Karlsruhe, Germany, 1608-1615. Dor : http:/ / dx.doi.org/10.1109/ICRA.2013.6630785

R. William Soukoreff and I. Scott MacKenzie. 2004. Towards a standard for pointing device evaluation, perspectives on 27 years of Fitts' law research in HCI. International Journal of Human-Computer Studies 61, 6 (Dec. 2004), 751-789. DoI: http:/ / dx.doi.org/10.1016/j.ijhcs.2004.09.001

Andrej-Nikolai Spiess and Natalie Neumeyer. 2010. An evaluation of R2 as an inadequate measure for nonlinear models in pharmacological and biochemical research: a Monte Carlo approach. BMC Pharmacology 10, 1 (2010), 6. Dor : http:/ /dx.doi.org/10.1186/1471-2210-10-6

Martin Spindler, Wolfgang Büschel, and Raimund Dachselt. 2012. Use Your Head: Tangible Windows for 3D Information Spaces in a Tabletop Environment. In Proceedings of the 2012 ACM International Conference on Interactive Tabletops and Surfaces (ITS '12). ACM, Cambridge, Massachusetts, USA, 245-254. Dor : http://dx.doi.org/10.1145/2396636.2396674

Stan Development Team. 2014. Stan: A C++ Library for Probability and Sampling, Version 2.4.0. (2014). http:// mc-stan.org/

Stan Development Team. 2015. Stan: A C++ Library for Probability and Sampling, Version 2.6.0. (2015). http://mc-stan.org/ 
Rainer Stiefelhagen. 2002. Tracking focus of attention in meetings. In Fourth IEEE International Conference on Multimodal Interfaces (ICMI '02). IEEE Comput. Soc, Pittsburgh, Pennsylvania, USA, 273-280. DoI: http://dx.doi.org/10.1109/ICMI.2002.1167006

Rainer Stiefelhagen and Jie Zhu. 2002. Head Orientation and Gaze Direction in Meetings. In CHI '02 Extended Abstracts on Human Factors in Computing Systems (CHI EA '02). ACM, Minneapolis, Minnesota, USA, 858-859. Dor : http://dx.doi.org/10.1145/506443.506634

Robert J. Teather and Wolfgang Stuerzlinger. 2011. Pointing at 3D Targets in a Stereo HeadTracked Virtual Environment. In Proceedings of the 2011 IEEE Symposium on 3D User Interfaces (3DUI 2011). IEEE, Singapore, 87-94. DoI : http:/ / dx.doi.org/10.1109/3DUI.2011.5759222

James R. Tresilian. 2005. Hitting a moving target: Perception and action in the timing of rapid interceptions. Perception $\mathcal{E}$ Psychophysics 67, 1 (2005), 129-149. DoI : http: / / dx.doi.org/10.3758/BF03195017

John W. Tukey. 1980. We Need Both Exploratory and Confirmatory. The American Statistician 34, 1 (1980), pp. 23-25. DoI: http:/ /dx.doi.org/10.1080/00031305.1980.10482706

Paul Van Schaik and Jonathan Ling. 2007. Design Parameters of Rating Scales for Web Sites. ACM Transactions on Computer-Human Interaction 14, 1 (May 2007), 35 pages. DoI: http:/ / dx.doi.org/10.1145/1229855.1229859

W3C. 2011. Scalable Vector Graphics (SVG). (2011). http://www.w3.org/TR/2011/ REC-SVG11-20110816/

A. T. Welford, A. H. Norris, and N. W. Shock. 1969. Speed and accuracy of movement and their changes with age. Acta Psychologica 30, 0 (1969), 3-15. DoI: http:/ / dx.doi.org/10.1016/0001-6918(69)90034-1

Thomas G. Whisenand and Henry H. Emurian. 1995. Some Effects of Angle of Approach on Icon Selection. In Conference Companion on Human Factors in Computing Systems (CHI '95). ACM, Denver, Colorado, USA, 298-299. Dor : http://dx.doi.org/10.1145/223355.223683

Thomas G. Whisenand and Henry H. Emurian. 1996. Effects of angle of approach on cursor movement with a mouse: Consideration of Fitt's law. Computers in Human Behavior 12, 3 (1996), 481-495. Dor: http://dx.doi.org/10.1016/0747-5632(96)00020-9

T. G. Whisenand and H. H. Emurian. 1999. Analysis of cursor movements with a mouse. Computers in Human Behavior 15, 1 (1999), 85-103. DoI : http:/ / dx.doi.org/10.1016/S0747-5632(98)00036-3

Hugh R. Wilson, Frances Wilkinson, Li-Ming Lin, and Maja Castillo. 2000. Perception of head orientation. Vision Research 40, 5 (Jan. 2000), 459-72. DoI : http:/ / dx.doi.org/10.1016/S0042-6989(99)00195-9 
Jonathan Wonner, Jérôme Grosjean, Antonio Capobianco, and Dominique Bechmann. 2011. SPEED : Prédiction de cibles. In 23rd French Speaking Conference on Human-Computer Interaction (IHM '11). ACM, Sophia Antipolis, France, 19:1-19:4. DoI: http:/ / dx.doi.org/10.1145/2044354.2044378

Chuang-Wen You, Yung-Huan Hsieh, and Wen-Huang Cheng. 2012. AttachedShock: Facilitating Moving Targets Acquisition on Augmented Reality Devices Using Goal-crossing Actions. In Proceedings of the 20th ACM International Conference on Multimedia (MM '12). ACM, Nara, Japan, 1141-1144. DoI : http:/ / dx.doi.org/10.1145/2393347.2396403

Chuang-Wen You, Yung-Huan Hsieh, Wen-Huang Cheng, and Yi-Hsuan Hsieh. 2014. AttachedShock: Design of a crossing-based target selection technique on augmented reality devices and its implications. International Journal of Human-Computer Studies 72, 7 (2014), 606-626. Dor:http://dx.doi.org/10.1016/j.ijhcs.2014.03.001

Shumin Zhai, Carlos Morimoto, and Steven Ihde. 1999. Manual and Gaze Input Cascaded (MAGIC) Pointing. In Proceedings of the SIGCHI conference on Human Factors in Computing Systems (CHI '99). ACM, Pittsburgh, Pennsylvania, USA, 246-253. Dor: http:/ / dx.doi.org/10.1145/302979.303053

Xinyong Zhang, Hongbin Zha, and Wenxin Feng. 2012. Extending Fitts' Law to Account for the Effects of Movement Direction on 2D Pointing. In Proceedings of the SIGCHI Conference on Human Factors in Computing Systems (CHI '12). ACM, New York, NY, USA, 3185-3194. DoI : http:/ / dx.doi.org/10.1145/2207676.2208737 
Annexe A

\section{RÉSUMÉ EN FRANÇAIS}




\section{CHAPITRE 1}

\section{INTRODUCTION}

La sélection de cibles en mouvement est une tâche courante et complexe dans l'interaction homme-machine (IHM) en général et en particulier dans le domaine de la réalité virtuelle (RV). La prédiction de l'action est une solution intégrale pour aborder les problèmes liés à l'interaction. Cependant, les techniques actuelles de prédiction sont basées sur le suivi continu des actions de l'utilisateur sans prendre en compte la possibilité que les actions d'atteinte d'une cible puissent avoir une composante importante préprogrammée-cette théorie est appelée la théorie du contrôle préprogrammé. En se basant sur la théorie du contrôle préprogrammé, cette thèse explore la possibilité de prédire les actions de sélection d'objets en mouvement, avant leur exécution. Plus spécifiquement, trois niveaux de prédiction d'action sont étudiés : 1) la performance des actions, mesurée par le temps de mouvement (TM) nécessaire pour atteindre une cible, 2) la difficulté prospective $(D P)$, qui représente la difficulté subjective de la tâche estimée avant son exécution, 3) l'intention de l'utilisateur, qui indique la cible visée par l'utilisateur. Dans le cadre de cette thèse, des modèles de prédiction d'intention sont développés à l'aide d'arbres de décision ainsi que des fonctions de classement-ces modèles sont évalués dans deux expériences en Rv présentées dans les chapitres 3 et 5 . Des modèles 1-D et 2-D de DP pour des cibles en mouvement basés sur la loi de Fitts sont développés et évalués dans une expérience en ligne, présentée dans le chapitre 4. Enfin, des modèles

de TM avec les mêmes caractéristiques structurelles des modèles de DP sont évalués dans l'expérience 3-D en Rv du chapitre 5. 


\section{ChAPITRE 2}

\section{ReVUe LITTÉRAIRE}

\subsection{Performance des tâches de sélection}

La performance des tâches de sélection dans l'IHM est usuellement étudiée en utilisant des modèles liés à la théorie de l'information, telles que les lois de Fitts [Fitts 1954] et de Hick-Hyman [Hick 1952; Hyman 1953].

\subsubsection{La loi de Hick-Hyman}

La loi de Hick-Hyman décrit la relation entre le temps de réaction (TR) requis pour faire un choix et le nombre $(n)$ et la probabilité $(p)$ de chacun des choix possibles (cf. équation (2.1)). Si tous les choix ont la même probabilité $(p=1 / n)$, l'équation (2.1) est réduite à l'équation (2.2). Le TR décrit juste le temps pour faire le choix, et non le temps de mouvement (TM) requis pour exécuter la sélection.

\subsubsection{La loi de Fitts}

La loi de Fitts décrit la relation entre le temps de mouvement (TM) requis pour atteindre une cible à l'index de difficulté (ID) de la cible, cf. équation (2.3). L'ID donne une mesure objective de la difficulté liée au pointage sur une cible comme une fonction du ratio entre la distance $(D)$ et la taille $(W)$ de la cible. Les deux formes les plus communes d'ID sont la formule originale de Fitts [1954], cf. équation (2.4), et la formule originale de Shannon, proposée par MacKenzie [1989], cf. équation (2.5).

\subsubsection{Formules en deux parties de la loi de Fitts et le gain contrôle-écran}

Welford et al. [1969] ont suggéré que le pointage peut être séparé en deux processus de contrôle : une partie motrice et une partie visuelle. Pour tenir compte de ces deux processus, Welford et ses collègues proposent la variation de la loi de Fitts présentée dans l'équation (2.6).

Shoemaker et al. [2012] ont trouvé que cette formule décrit le TM des tâches de pointage avec différents niveaux de gain contrôle-écran $(G$, le ratio de la vitesse du curseur par rapport 
à la vitesse d'entrée). De plus, ils ont observé que le ratio $k=c / b$ incrémentait linéairement avec $G$, mais aucune mention n'a été faite sur les relations entre $a$ et $G, b$ et $G$, et $c$ et $G$.

Formule alternative en deux parties. Hoffmann and Chan [2012] reformulèrent le modèle en deux parties de Welford, cf. (2.8). Dans celle-ci, le terme $b^{\prime} \sqrt{D}$ indique que la partie motrice $\mathrm{du}$ mouvement de pointage et principalement balistique, tandis que le terme $c I D$ indique que la partie visuelle du mouvement suit l'ID de Fitts [Hoffmann and Chan 2012].

Les équations (2.6) et (2.8) sont approximativement équivalentes pour certains rangs de D. Cependant, selon Hoffmann and Chan [2012], l'équation (2.8) est préférable à la l'équation (2.6), car les logarithmes doivent seulement être calculés sur des quantités sans unité, ce qui n'est pas le cas de $D$ et $W$.

\subsubsection{Formules 2-D de la loi de Fitts pour des tâches de pointage statiques}

La loi de Fitts est intrinsèquement $1-D$, mais est utilisée en pratique dans des tâches 2-D, ignorant les effets de l'angle (thêta) et la forme de la cible sur le TM. Une tâche de sélection 2-D avec une cible bivariée (i.e., avec largeur $W$ et hauteur $H$ différentes) est montrée dans la figure 2.1.

Cependant, plusieurs travaux ont pour étude l'effet de thêta sur TM. Dans la littérature, il y a trois modifications de l'ID de Shannon qui modélisent l'effet de thêta :

- La dérivation empirique de Murata and Iwase [2001] avec une constante empirique c, cf. équation (2.10),

- le modèle d'Appert et al. [2008] qui modélise aussi des cibles avec des $W$ et $H$ différentes, cf. équation (2.11)

- et la formule d'ID par Grossman and Balakrishnan [2004] qui permet de modeler l'effet de l'angle séparément pour chacune des dimensions de la cible (formulée et testée en 3-D mais réduite ici en 2-D), cf. équation (2.12).

Les équations (2.10)-(2.12) représentent la contribution de thêta de trois façons différentes. $\mathrm{Vu}$ que ces dernières ont été dérivées empiriquement ou par analogie, cela pose la question sur la forme réelle de la contribution de thêta.

En termes de facilité d'utilisation, les équations (2.10) et (2.11) sont les plus simples, nécessitant juste des procédures telle que la méthode linéaire des moindres carrées. Au contraire, l'équation (2.12) nécessite une procédure plus spécialisée telle que la méthode non-linéaire des moindres carrées. Dans le cas de l'équation (2.12), l'ajustement du modèle aux données en terme de coefficient de corrélation $\left(R^{2}\right)$ peut être artificiellement plus élevé que les équations (2.10) et (2.11) dû aux $2 \times \#(\theta)$ termes additionnels représentés par $f_{W}(\theta)$ et $f_{H}(\theta)$. Plus important encore, l'usage de $R^{2}$ serait inadéquat pour évaluer l'ajustement des modèles non-linéaires [Spiess and Neumeyer 2010]. Ces deux derniers points sont critiques 
dans le statu quo de la littérature de Fitts, où l'ajustement des modèles est habituellement évalué en utilisant $R^{2}$.

\subsubsection{Formules 3-D de la loi de Fitts pour des tâches de pointage statiques}

A la connaissance de l'auteur, le seul travail formulant des modèles d'ID spécifiques pour 3-D est celui de Grossman and Balakrishnan [2004]. Ce modèle est une extension de l'équation (2.12) qui ne tient pas compte de tous les paramètres spatiaux des tâches 3-D, tel qu'il est montré dans la figure 2.2.

\subsubsection{Formules de la loi de Fitts pour des tâches de pointage sur des cibles en mouve- ment 1-D}

La recherche sur la loi de Fitts pour des tâches de pointage sur des cibles en mouvement reste rare comparée aux tâches de pointage sur des cibles statiques. Jagacinski et al. [1980], pionniers dans ce type d'étude, trouvèrent que l’ID de Fitts était faiblement corrélé avec le TM requis pour atteindre des cibles en mouvement en utilisant une technique de contrôle positionnelle. A la place, ils proposèrent une formule avec des caractéristiques analogues à celles de formules en deux parties. Leur formule générale se différencie d'autres modèles présentés dans cette thèse car non composés de logarithmes ou racines carrées, cf. (2.15).

Une particularité de l'étude de Jagacinski, est que les essais de pointage étaient considérés réussis lorsque le curseur restait sur la cible au moins $350 \mathrm{~ms}$. Ce temps de capture $\left(T_{c}\right)$ n'est, sans doute, pas approprié pour des périphériques d'entrée différents au joystick utilisé dans leur expérience.

Hoffmann [1991] formula un ID pour la sélection des cibles en mouvement plus proche en forme à l'ID originale de Fitts, cf. (2.17). Le principal problème de cette formule est que le domaine des valeurs de $K$ dépend du plan expérimental. Ainsi, une fois $K$ calculé, l'ensemble des conditions expérimentales $(D, W, V)$ qui peuvent être mathématiquement modélisées par l'équation (2.17) est restreint, cf. équations (2.19) et (2.20).

\subsubsection{Formules de la loi de Fitts pour des tâches de pointage sur des cibles en mouve- ment 2-D}

Al Hajri et al. [2011] ont suggèré de combiner l'équation (2.17) avec, notamment, l'équation (2.12) pour modéliser des tâches 2-D, ce qui résulte dans l'équation (2.21). Même si cette dernière peut modéliser des cibles en mouvement en 2-D, les études d'Al Hajri et al. [2011], incluent seulement des conditions où les cibles se déplacent directement vers le pointeur ou s'éloignent directement du pointeur, i.e. $\phi \in\{0,180\}$, même si $\theta \in[0,360)^{\circ}$.

\subsection{Difficulté subjective}

En dehors du TM, l'ID a aussi été corrélé avec la difficulté subjective [Delignières and Famose 1992; Slifkin and Grilli 2006; Grilli 2011; Chan and Hoffmann 2013]. Il y a deux types de 
difficulté subjective (qui seraient aussi corrélées entre elles) dans la littérature :

1. La difficulté prospective $(D P)$, ou estimée, qui désigne les évaluations subjectives précédant l'exécution de la tâche.

2. La difficulté perçue, qui désigne les évaluations subjectives succédant l'exécution de la tâche.

A la connaissance de l'auteur, il n'y a aucune étude corrélant directement la difficulté subjective et l'ID pour des tâches de sélection de cibles en mouvement.

\subsection{Prédiction d'intention}

La prédiction d'intention (entendue ici comme la prédiction de cibles voulues) a été proposée dans la sélection statique pour résoudre les problèmes de clutter et superposition de cibles. Cependant, les techniques utilisées dans la sélection statique ne sont pas appropriées pour la prédiction des cibles en mouvement. Pour ces dernières, il existe deux techniques plus prometteuses : les fonctions de scoring et le regard.

\subsubsection{Les fonctions de scoring}

De Haan et al. [2005] et Ortega [2013] ont démontré la possibilité de prédire les cibles voulues dans des scènes complexes en utilisant des fonctions de scoring. En général, les étapes pour utiliser ces fonctions peuvent être décrites ainsi :

1. Filtrage des cibles : un sous-ensemble de cibles qui satisfont un critère donné est choisi.

2. Attribution des scores aux cibles: Les scores des cibles qui satisfont le critère sont incrémentés, et décrémentés le cas échéant.

3. Marquage : la cible avec le score le plus élevé est marquée.

4. Sélection : l'utilisateur confirme la sélection de la cible marquée ou continue à mouvoir le curseur jusqu'à ce que la cible voulue soit marquée.

De Haan et al. [2005] utilisèrent un critère basé sur la distance angulaire entre la cible et le curseur, tandis qu'Ortega [2013] basa son critère sur la distance euclidienne entre le curseur et la cible. 


\subsubsection{Le regard}

L'objet de regard est considéré comme un indicateur de ce qui est " au sommet de la pile » d'un processus cognitif [Just and Carpenter 1976]. Le regard précède le mouvement des mains [Johansson et al. 2001] et est composé par l'orientation de la tête et l'orientation des yeux par rapport à la tête. Le tracking des yeux est une solution idéale pour prédire l'intention, mais des difficultés techniques rendent son usage limité dans des environnements virtuels immersifs de type CAVE. Le tracking de tête, au contraire, est souvent disponible dans ce type de système et a été utilisé avec succès pour prédire la direction du regard, et plus spécifiquement la cible voulue [Stiefelhagen and Zhu 2002; Stiefelhagen 2002; Nickel and Stiefelhagen 2003]. 


\section{ChAPITRE 3}

\section{L'INTENTION DANS LA SÉLECTION NON-DIRIGÉE DE CIBLES EN MOUVEMENT 3-D}

Basée sur l'hypothèse que les utilisateurs forment leurs intentions en minimisant leur effort prospectif, décrit par l'ID, au chapitre 1, l'hypothèse que l'intention peut être prédite en utilisant l'ID dans des taches de sélection non-dirigée a été formulée.

Malheureusement, comme ont été montrées dans le chapitre 2, les mesures existantes d'ID pour la sélection de cibles en mouvement ne sont pas compatibles avec le cadre théorique DP-ID. Le modèle de Hoffmann [1991] pour les cibles en mouvement, présenté dans l'équation (2.17), et son extension en 2-D par Al Hajri et al. [2011], présentée dans l'équation (2.21), requièrent le pourcentage de captures réussites, ce qui est inconnu dans l'action prospective, faute de quoi l'application de ces modèles résulte sur des calculs plus complexes et non généralisables.

D'autre part, les études de Jagacinski et al. [1980] révèlèrent que même si l'ID de Fitts fut peu corrélé avec le TM des cibles en mouvement, les résultats furent à peine meilleurs dans des conditions avec des vitesses de cible identiques. De plus, la corrélation entre TM et $I D$ s'avera plus élevée dans des conditions où la vitesse des cibles était plus lente. Tenant compte de ces limitations, l'hypothèse selon laquelle l'ID peut prédire l'intention dans de tâches de sélection de cibles en mouvement avec une seule $V$ relativement basse, est évaluée dans ce chapitre. Similaire au modèle de Jagacinski et al. [1980], équation (2.14), utilisant $D$ et $W$ séparément pour prédire $T M$, la précision des prédictions basées sur l'ID est comparée à celle de ces composantes $D$ et $W$.

L'utilité potentielle de $D$ et $W$, cependant, suppose que l'utilisateur est libre de choisir sa cible, autrement dit, elle est limitée aux tâches non-dirigées. C'est pourquoi, ces mesures spécifiques à la tâche sont complémentées avec des caractéristiques généralisables qui peuvent aussi être utilisées dans des tâches dirigées. Plus spécifiquement, ces caractéristiques généralisables proposées, consistent en des états utilisateur-cibles.

Les contenus de ce chapitre sont basés sur des publications par l'auteur et certains membres du jury. Plus précisément, l'analyse d'ID, D et $W$ parue dans Casallas et al. [2013], et l'extension avec des caractéristiques généralisables parue dans Casallas et al. [2014]. 


\subsection{Hypothèses}

\subsubsection{Caractéristiques spécifiques à la tâche pour la prédiction d'intention dans des tâches de sélection non-dirigée de cibles en mouvement}

H1. L'ID peut prédire l'intention de façon précise dans des tâches de sélection non-dirigée de cibles en mouvement.

\subsubsection{Caractéristiques généralisables pour la prédiction d'intention dans des tâches de sélection non-dirigée de cibles en mouvement}

H2. Les caractéristiques relatives utilisateur-cible peuvent prédire l'intention de façon précise dans des tâches de sélection non-dirigée des cibles en mouvement.

H3. L'utilisation simultanée des caractéristiques généralisables et spécifiques à la tâche résultent en des prédictions d'intention plus précises que leur utilisation séparée.

\subsection{Etude pilote}

Avant la mise en place de l'expérience, une étude pilote évalua le meilleur algorithme, ainsi que le temps optimal pour mesurer les caractéristiques $D$ et $W$, pour prédire l'intention dans des tâches de sélection non-dirigée de cibles en mouvement. Les algorithmes évalués furent : le classifieur bayésien naïf [Mitchell 1997, 177-180], les réseaux de neurones [Bishop 2006, 225-290] et les arbres de décision c4.5 [Quinlan 1993]. L'étude pilote fut conduite avec Ashwin S. Natarajan et Keji Hu, dans le cadre du projet de cours de machine learning (cs 573) à Iowa State University au printemps 2012.

Les résultats favorisèrent l'utilisation de l'algorithme c4.5, dû principalement à la facilité pour comprendre les règles produites. De plus, les résultats prônent la mesure des caractéristiques $\mathrm{D}$ et $\mathrm{W}$ en début de chaque essai. L'algorithme c4.5 est décrit dans les équations (3.1)-(3.4).

\subsection{Méthode}

\subsubsection{Participants}

Vingt-six personnes âgées de 23 à 47 ans participèrent à l'étude. Il y eut 18 hommes et 8 femmes ; seulement deux sujets étaient gauchers. 


\subsubsection{Matériel}

L'expérience fut développée en vR Jugglua [Pavlik and Vance 2012], et déployée dans le MOVE, un environnement virtuel (Ev) de type CAve à 4 faces ( 3 murs et sol) de $3 \times 3 \times 2.67 \mathrm{~m}$. Des projecteurs stéreo passifs Infitec [Jorke et al. 2008] avec une résolution de $1160 \times 1050$ par face furent utilisés. Quatre caméras ART suivirent la pose (position, $P$, et orientation, $Q$ ) de la tête et le wand de l'utilisateur, permettant à ce dernier une perception et interaction 3-D adéquate de l'environnement virtuel.

Un système de coordonnées " $y$-up » fut utilisé, avec son origine placé au niveau du sol au milieu de l'Ev, $z$ décrémentant vers le mur frontal et $x$ incrémentant vers le mur de droite. La configuration expérimentale peut être appréciée dans la figure 3.2.

\subsubsection{Procédure}

Après avoir rempli un questionnaire court, le participant a été dirigé vers un repère circulaire $(r=0.25 \mathrm{~m})$ localisé au centre du move $(0,0,0)$, faisant face au mur frontal de ce dernier, où il completa une série de tâches de sélection d'objets. Lors de chaque essai, fut présenté au participant un ensemble horizontal de sphères virtuelles de tailles différentes, commençant face à lui et volant dans sa direction en $z$. Toutes les sphères avaient la même texture mise à l'échelle de la sphère. On demanda au participant de toucher chaque sphère en étirant son bras juste pour toucher la sphère ; au lieu d'attendre les sphères avec le bras étiré. Chaque sphère disparaissait lors du toucher, ou au moment de dépasser la tête du participant par plus de $0.5 \mathrm{~m}$. L'essai finissait quand le participant eut touché toutes les sphères ou quand les sphères restantes eurent passé sa tête.

\subsubsection{Protocole}

Un protocole factoriel, intra-sujets a été utilisé, avec trois blocs d'essais. Dans chaque essai, toutes les sphères apparaissaient $0.3 \mathrm{~m}$ en-dessous et $5 \mathrm{~m}$ en face de la tête du participant $\left(\left(P_{i, y}=P_{h, y}-0.3, P_{i, z}=-5\right)\right)$.

Lors du premier bloc, chaque essai avait une seule sphère, se déplaçant à une vitesse de $2.5 \mathrm{~m} / \mathrm{s}$ en $z$. Les facteurs expérimentaux étaient le rayon de la sphère $\left(r_{1} \in\{0.1,0.2\}\right)$ et la position de la sphère (gauche : $P_{1, x}=0.5$, centre : $P_{1, x}=0$, et droite : $P_{1, x}=0.5$ ). Chacune des six conditions fut présentée au participant dans un ordre aléatoire jusqu'à ce qu'il complète cinq essais par condition (30 au total). Le premier bloc fut planifié juste pour l'entrainement, de façon à ce que les participants puissent se familiariser avec l'environnement et la tâche.

Après avoir complété le premier bloc d'essais, le nombre de sphères fut incrémenté à deux et la vitesse fut décrémentée à $1.5 \mathrm{~m} / \mathrm{s}$ en $z$. Les sphères furent placées à $0.5 \mathrm{~m}$ de distance en $x$ les unes des autres, mais toutefois la paire pouvait apparaître vers la gauche $\left(P_{1, x}=-0.5, P_{2, x}=0\right)$, vers la droite $\left(P_{1, x}=0, P_{2, x}=0.5\right)$, ou au centre $\left(P_{1, x}=-0.25, P_{2, x}=0.25\right)$ 
par rapport à l'utilisateur (cf. figure 3.3). Les facteurs expérimentaux étaient le rayon de la sphère $\left(r_{i} \in\{0.1,0.2\}\right.$ ) et la position de l'ensemble (gauche, centre, ou droite). Chacune des 12 conditions fut présentée au participant dans un ordre aléatoire jusqu'à ce qu'il complète cinq essais par condition (60 au total).

Il y eut un troisième bloc avec trois sphères, mais ce dernier n'est pas présenté. L'analyse d'une tâche plus complexe avec une, trois et six sphères et vitesses différentes est présentée dans le chapitre 5.

\subsubsection{Discussion}

Les résultats sont synthétisés selon les hypothèses de l'étude, chacun suivi par une discussion.

\subsubsection{Caractéristiques spécifiques à la tâche pour la prédiction d'intention dans des tâches de sélection non-dirigée de cibles en mouvement}

Compte tenu que les arbres de décision à caractéristiques spécifiques de la tâche ont été construits en se basant seulement sur la position initiale du wand de l'utilisateur et la taille et position initiales des sphères, les prédictions eurent des précisions relativement hautes, comparés aussi bien aux résultats obtenus par chance que par un prédicteur sans-règle (zero-rule). C'est probable, néanmoins, que la précision faiblisse si le nombre de cibles est incrémenté, mais on peut espérer que la précision soit toujours meilleure que la chance et le prédicteur sans-règle.

H1. L'ID peut prédire l'intention de façon précise dans des tâches de sélection non-dirigée de cibles en mouvement. Partiellement validée.

Même si l'ID servit comme une bonne caractéristique spécifique pour prédire la cible voulue, l'utilisation des rayons $\left(r_{1}, r_{2}\right)$ des sphères, résulta en une précision équivalente $(\sim 72 \%)$. Ceci suggère que la taille de la cible résulte dans un gain d'information plus important que la distance initiale à la cible et suggère une stratégie très basique des participants où la distance ne joue pas de rôle important dans le choix des cibles.

\subsubsection{Caractéristiques généralisables pour la prédiction d'intention dans des tâches de sélection non-dirigée de cibles en mouvement}

H2. Les caractéristiques relatives utilisateur-cible peuvent prédire l'intention de façon précise dans des tâches de sélection non-dirigée des cibles en mouvement. Partiellement validée. 
Les caractéristiques relatives tête-cible et wand-cible, $\overline{\Delta d o t}$ et $\overline{\Delta D}$ prédirent les cibles voulues avec succès dans les tâches étudiées. Etant combinées, les caractéristiques résultèrent en une précision de $\sim 72 \%$ pour prédire les cibles voulues, ce qui est significativement meilleur que la caractéristique $\overline{\Delta D}$ isolée $(\triangle a c c=3.31 \%)$, et la fonction de scoring d'Ortega [2013] $(\triangle a c c=3.63 \%)$.

La caractéristique relative tête-cible, $\Delta d o t$, fut utile non seulement pour la prédiction $\mathrm{d}$ 'intention, mais aussi pour établir une fenêtre de temps pour moyenner les caractéristiques généralisables. Actuellement, la fenêtre est établie empiriquement à partir du graphique de $\Delta$ dot vs. $t$ (cf. figure 3.5).

H3. L'utilisation simultanée des caractéristiques généralisables et spécifiques à la tâche résultent en des prédictions d'intention plus précises que leur utilisation séparée. Validée.

L'utilisation des caractéristiques $\overline{\Delta d o t}$ et $\overline{\Delta D}$ conjointement avec $r_{1}$ et $r_{2}$ améliora la précision des prédictions des cibles voulues. Cet ensemble combiné de caractéristiques performa significativement mieux que tous les autres ensembles de caractéristiques, surpassant le meilleur ensemble de caractéristiques spécifiques à la tâche, $\left\{r_{1}, r_{2}\right\}$, par presque $7 \%$, et le meilleur ensemble de caractéristiques généralisables, $\{\overline{\Delta d o t}, \overline{\Delta D}\}$, par environ $6 \%$. 


\section{CHAPITRE 4}

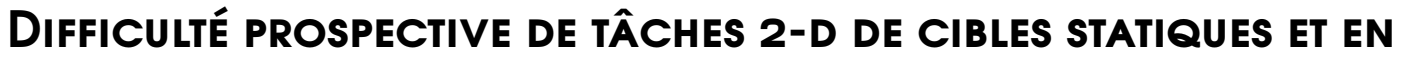 MOUVEMENT}

Dans le chapitre 3, on a évalué l'hypothèse présentée dans le chapitre 1 , selon laquelle l'ID était prédictif de l'intention dans des tâches non-dirigées de sélection de cibles en mouvement, basé sur la prémisse que les utilisateurs forment leurs intentions en minimisant leur effort prospectif, décrit par l'ID. Dû aux déficiences des mesures existantes d'ID pour la sélection des cibles en mouvement, le chapitre 3 recourut à l'utilisation d'une formule d'ID pour des cibles statiques, ainsi que ses composantes $D$ et $W$ séparément. En accord avec Jagacinski et al. [1980] pour le TM des cibles en mouvement, les résultats révélèrent que cet $I D$ est limité en utilité pour prédire l'intention dans la sélection de cibles en mouvement, indiquant par conséquent son caractère inadéquat en tant que mesure de $D P$ pour ce genre de tâche.

Pour adresser ces limitations, ce chapitre essaie d'étendre le paradigme $D P-I D$ aux tâches de sélection de cibles en mouvement. Le modèle proposé est formulé et évalué initialement dans de tâches 2-D pour éviter les problématiques inhérentes à l'interaction 3-D, telle que la perception incorrecte de la profondeur, ayant toutefois l'extensibilité en 3-D en tête. Une tâche générale de pointage de cibles en 2-D est présentée dans la figure 4.1, avec ces attributs relevants.

Pour atteindre ce but, le chapitre 4 introduit trois modèles basés sur l'ID de Fitts pour la $D P$ de pointer sur des cibles statiques en 2-D, et des cibles en mouvement en 1-D et 2-D. Les formules sont présentées dans les sections 4.1.1-4.1.3.

\subsection{Formules}

\subsubsection{Formule de DP pour des tâches 2-D de sélection de cibles statiques}

La sélection 2-D de cibles statiques fait référence aux tâches où l'angle initiale $\theta \in[0,360)^{\circ}$. Pour ce type de tâche, le modèle proposé dans ce chapitre sépare l'effet de l'angle de la cible $(\theta)$ du terme $I D=\log _{2}(2 D / W)$, tel que 


$$
D P=a_{\theta}+b \log _{2}\left(\frac{2 D}{W}\right),
$$

où $a_{\theta}$ et $b$ sont des coefficients déterminés empiriquement; $a_{\theta}$ est l'intercepte par-angle $\theta$. Cette formule est basée sur la formule originale de Fitts pour TM, ${ }^{1}$ et les extensions 2-D de l'ID par Murata and Iwase [2001], Appert et al. [2008] et Grossman and Balakrishnan [2004]. Cette équation est développée dans la section 4.2.2.

\subsubsection{Formule de DP pour des tâches 1-D de sélection de cibles en mouvement}

La sélection 1-D de cibles en mouvement fait référence aux tâches où l'angle initial de la cible $\theta \in\{0,180\}^{\circ}$, et $1^{\prime}$ angle de mouvement de la cible $\phi \in\{0,180\}^{\circ}$. Pour ce type de tâche, le modèle proposé dans ce chapitre est basé sur le modèle en deux parties de Hoffmann and Chan [2012] avec des coefficients par vitesse $(V)$, tel que

$$
D P=a_{V}+b_{V} \sqrt{D}+c_{V} \log _{2}\left(\frac{2 D}{W}\right),
$$

où $a_{V}, b_{V}$ et $c_{V}$ sont des coefficients dérivés empiriquement. Cette formule, basée sur l'analogie entre le gain contrôle-écran $(G)$ et $V$, est inspirée par le travail de Shoemaker et al. [2012], qui trouvèrent que les modèles en deux parties peuvent être utilisés pour décrire le pointage sur des cibles statiques avec des niveaux différents de $G$.

Cette formule est développée dans la section 4.2.3. De plus, comme montrés dans la section 4.4.2.2, les coefficients $a_{V}, b_{V}$ et $c_{V}$ sont vraisemblablement corrélés linéairement avec la vitesse $V$ de la cible.

\subsubsection{Formule de DP pour des tâches 2-D de sélection de cibles en mouvement}

La sélection 2-D de cibles en mouvement fait référence aux tâches où l'angle de mouvement de la cible $\phi \in[0,360)^{\circ}$, quelque soit l'angle initiale $\theta$ de la cible. Pour ce type de tâche, le modèle proposé est une extension du modèle 1-D pour des cibles en mouvement, séparant la distance initiale vers la cible $(D)$ en deux composantes, telles que

$$
\begin{gathered}
D P=a_{V}+b \sqrt{D_{s}}+c_{V} \sqrt{D_{m}}+d \log _{2}\left(\frac{2 D_{m}}{W}\right) \\
D_{s}=|D \sin \phi| \\
D_{m}=|D \cos \phi|,
\end{gathered}
$$

\footnotetext{
${ }^{1}$ Le choix de formule d'ID de Fitts est expliqué dans la section 4.2.1.
} 
où $a_{V}, b, c_{V}$ et $d$ sont des coefficients dérivés empiriquement; $a_{V}$ et $c_{V}$ sont des coefficients par vitesse $(V)$. Le développement de cette formule est aussi bien analytique, comme exploré dans la section 4.2.4, et empirique, comme exploré dans la section 4.4.2.2. De plus, comme montrés dans la section 4.4.2.2, $a_{V}$ et $c_{V}$ sont vraisemblablement corrélés linéairement avec la vitesse $V$ de la cible.

\subsection{Méthode}

\subsubsection{Matériel}

Pour évaluer les modèles proposés, une expérience a été implementée en utilisant des scalable vector graphics (svG) [W3C 2011] et déployé dans un questionnaire Qualtrics en ligne.

\subsubsection{Participants}

Des participants ont été recrutés en distribuant le lien du questionnaire sur des réseaux sociaux. Quarante-neuf sur 83 répondants répondirent à la totalité du questionnaire-seulement les réponses complètes furent prises en compte. Vingt-et-un participants reportèrent être étudiants, et il a été déterminé, en utilisant des horodatages, que 25 participants venaient du continent américain, un de l'Inde et le reste de l'Europe ou l'Afrique.

Les participants étaient âgés de 20 à 48 ans $(M=29.65, M d n=29)$; il y eut 14 femmes et 35 hommes. La plupart de répondeurs reportèrent être droitiers (45) ; aucun répondeur reporta être ambidextre.

Seulement 41 réponses furent retenues, les critères de sélection sont expliqués dans la section 4.3.5.

\subsubsection{Procédure}

En ouvrant le lien du questionnaire, le participant fut demandé d'accepter un formulaire de consentement pour participer dans l'étude. En acceptant, une animation d'un objet en mouvement était présentée. Après la lecture de cette animation, le participant devait confirmer si l'objet en mouvement était visible sur l'écran-cette confirmation servit à valider le navigateur. Après avoir validé celui-ci, le participant remplissait un questionnaire court concernant son background et son information démographique.

Subséquemment, on présenta deux blocs de questions. Dans chaque question, le participant devait évaluer la difficulté prospective requise pour toucher, le plus vite possible, une cible circulaire à partir d'un point de départ donné, sans toucher l'écran ni déplacer les doigts. On conseilla au participant de maximiser la fenêtre du questionnaire avant le début de chaque bloc pour éviter les distractions et réduire le défilement du navigateur. Dans le premier bloc, les cibles étaient statiques; dans le deuxième bloc les cibles étaient en mouvement. 
Chaque question était présentée dans une nouvelle page et était évaluée en utilisant des boutons radio, comme conseillé par Van Schaik and Ling [2007]. Chaque question était évaluée en utilisant une échelle de type Likert à neuf points, où 1 était libellé " Très facile " et 9 était libellé « Très difficile ».

Pour donner un point de comparaison aux évaluations, une image-type (« l'ancre »), correspondant à la difficulté intermédiaire (5), fut donnée avant chaque bloc ainsi que dans l'entête de chaque question. La même image ancre avec une cible statique fut utilisée pour les questions avec des cibles statiques et les questions avec des cibles en mouvement.

Pour éviter que le participant tente d'exécuter la tâche de pointage, le curseur de la souris disparaissait en passant sur chaque image. Il n'y avait pas de limite pour chaque réponse et, dans le cas des cibles en mouvement, les animations pouvaient être relues à la discrétion du participant. La capture d'écran de l'une des questions avec une cible en mouvement est présentée dans la figure 4.3.

\subsubsection{Protocole}

Un protocole factoriel, intra-sujets à été utilisé, avec deux blocs d'essais. Le premier bloc consistait en des cibles statiques. Les facteurs expérimentaux étaient la distance $D \in\{200,400\}$ pixels (px) ; le rayon des cibles $r \in\{20,40,80\}$ px ; et l'angle de la cible, $\theta \in\{-45,0,45\}^{\circ}$. Notez que l'angle $\theta$ était mesuré dans le sens des manettes de l'horloge, en accord avec le système de coordonnées d'écran "y-down ». Chacune des 18 conditions fut présentée une fois au participant dans un ordre aléatoire. Un exemple de cette tâche, avec ses paramètres, est montré dans la figure 4.4.

Le deuxième bloc consistait en des cibles en mouvement. Les facteurs étaient la distance initiale $D \in\{200,400\}$ px ; le rayon des cibles, $r \in\{20,40,80\}$ px ; la vitesse des cibles, $V \in\{200,400,800\} \mathrm{px} / \mathrm{s}$; et la direction initiale du mouvement, $\phi \in\{-45,0,45\}^{\circ}$. L'angle initial $\theta$ de la cible était toujours $0^{\circ}$. Chacune des 54 conditions fut présentée au participant une fois, dans un ordre aléatoire. Un exemple de cette tâche, avec ses paramètres, est montré dans la figure 4.5.

L'image ancre des tâches avec des cibles statiques et avec des cibles en mouvement était $\left(D=200 \mathrm{px}, r=20 \mathrm{px}, \theta=0^{\circ}, V=0 \mathrm{px} / \mathrm{s}\right)$. Le choix de la même ancre pour les cibles statiques et en mouvement fut basé sur la notion selon laquelle la sélection des cibles en mouvement est une extension de la sélection des cibles statiques.

\subsection{Discussion}

Les résultats sont synthétisés par rapport aux hypothèses formulées, chacune suivie par une discussion. 


\subsubsection{Prospective difficulty of static-target selection}

H1. Le modèle d'ID de Fitts avec un intercepte par angle, décrit dans l'équation (4.11), modélise la difficulté prospective des tâches 2-D de sélection de cibles statiques mieux que le modèle classique d'ID de Fitts avec un intercepte unique, décrit dans l'équation (4.1). Partiellement validée.

Le modèle à intercepte fixe de l'équation (4.1) et le modèle à intercepte par angle de l'équation (4.11) étaient bien ajustés aux données, avec des intervalles crédibles de $R^{2}=$ $[0.94,0.99]$ et $R^{2}=[0.97,0.99]$. Cependant, comparé aux résultats du modèle d'intercepte fixe, présentés dans le tableau 4.1, les résultats du modèle à intercepte par angle présentés dans le tableau 4.2 rendirent des estimés légèrement meilleurs et plus précis de $\sigma$ et $R^{2}$. La différence en précision, $M d n\left(\sigma_{1}-\sigma_{2}\right)=0.07$, 995\% HDI $[-0.04,0.2]$ représente, pourtant, moins de $1 \%$ du rang total de l'échelle (1-9).

Même si le modèle 2-D est " significativement meilleur » en termes statistiques, ${ }^{2}$ la différence en précision entre les deux modèles n'est pas pratiquement significative. L'importance du modèle, toutefois, demeure dans sa flexibilité et extensibilité à d'autres angles. C'est pourquoi $H 1$ est partiellement validée.

Dans tous les cas, le fait que l'ID fut linéairement corrélé avec $D P$, supporte l'utilisation de l'échelle à 9 points choisie pour l'expérience. Ce point est crucial pour valider les évaluations de $D P$ pour la sélection de cibles en mouvement utilisant cette échelle.

\subsubsection{Difficulté prospective de la sélection de cibles en mouvement}

H2. . Le modèle en deux parties de Hoffmann avec des coefficients par vitesse, décrit dans l'équation (4.18), modélise précisément la difficulté prospective dans des tâches 1-D de sélection de cibles en mouvement. Validée.

Le modèle 1-D de sélection de cibles en mouvement de l'équation (4.18) s'ajusta très bien aux données, avec des valeurs crédibles de $R^{2}=[0.92,0.99]$ (cf. tableau 4.3), et des lignes de régression qui suivent les données de près sans générer des motifs non-aléatoires dans les résidus (cf. figure 4.12). Ce résultat était prévisible, étant donnée la similarité entre l'équation (4.18) et le modèle " général » de Jagacinski (équation (2.15)), ainsi que l'analogie $G-V_{\text {cible, }}$ présentée dans la section 4.2.3.

H2.1. Les coefficients $a_{V}, b_{V}$ et $c_{V}$ de l'équation (4.18) varient linéairement avec $V$. Validée.

\footnotetext{
${ }^{2}$ Ce qui peut être vérifié en utilisant un test $F$ classique, non montré.
} 
Les résultats suggèrent que les coefficients $a_{V}, b_{V}$ et $c_{V}$ de l'équation (4.18) varient linéairement avec $V$. L'analogie avec le gain contrôle-écran, pour lequel ces coefficients semblent aussi varier linéairement, soutient aussi cette hypothèse. En utilisant aussi les intervalles crédibles du tableau 4.5, on peut prédire $D P$ pour d'autres $V$, tout en propageant l'incertitude sur ces estimations.

H3. Le modèle en trois parties, avec des coefficients par vitesse et intercepte par vélocité, décrit dans l'équation (4.20), modélise de façon précise la difficulté prospective des tâches 2-D de sélection de cibles en mouvement. Validée.

On a déterminé que le modèle en trois parties avec des coefficients par-vitesse et intercepte par-vélocité de l'équation (4.20) était vainement complexe pour les données évaluées ; cependant, le modèle avec intercepte et coefficient c par vitesse, décrit dans l'équation (4.28) s'ajusta très bien aux données, avec des valeurs crédibles de $R^{2}=[0.96,0.98]$ (cf. tableau 4.7), et des lignes de régression qui suivent les données de près sans générer des motifs non-aléatoires dans les résidus (cf. figure 4.13).

Etant donné que l'équation (4.28) est comprise dans l'équation (4.20), ceci implique que la dernière s'ajuste aussi bien aux données, par conséquent $H 3$ est validée. Même si le modèle plus simple était suffisant pour ces données, l'équation (4.20) ne devrait pas être ignorée dans des analyses futures, spécialement celles avec une variété plus importante d'angles, où l'asymétrie angulaire pourrait avoir un effet plus large.

H3.1. Dans l'équation (4.20) les coefficients $b_{V}, c_{V}$ et $d_{V}$ varient linéairement avec $V$, ainsi que les coefficients $a_{V \times \phi}$ dans le même angle $\phi$. Partiellement validé.

Les résultats suggèrent que les coefficients $a_{V}$ et $c_{V}$ de l'équation (4.28) croissent linéairement avec $V$. Contrairement à l'hypothèse, $b$ et $d$ étaient presque constants parmi les $V$ évalués. Pour $b$, ceci suggère que le mouvement balistique prospectif parallèle au mouvement de la cible est affecté à minima par $V$. Pour $d$, ceci suggère que l'effet de $W$ ne change pas entre les $V$ (cf. graphe d'interaction de la figure 4.11). En utilisant les intervalles crédibles présentés dans le tableau 4.9, ceci permet de prédire $D P$ en utilisant d'autres $V$, tout en propageant l'incertitude sur ces estimées. 


\section{ChAPITRE 5}

\section{Performance et intention dans la sélection dirigée de Cibles en MOUVEMENT EN 3-D}

Dans les chapitres 1-3 on formula l'hypothèse que l'ID était prédictif de l'intention de l'utilisateur dans des tâches de sélection de cibles en mouvement, basé sur la prémisse que les utilisateurs forment leurs intentions en minimisant leur effort prospectif, décrit par l'ID. Les résultats du chapitre 3 révélèrent, pourtant, que le fait de séparer les composantes $W$ et $D$ était plus effectif pour prédire l'intention, similaire aux modèles proposés dans le chapitre 4, qui séparent $D$ de l'ID pour prédire la $D P$.

Cette similarité suggère que les modèles proposés dans le chapitre dernier peuvent aussi être prédictifs de l'intention de l'utilisateur, mais cette relation doit être explorée plus en profondeur. En particulier, pour prédire l'intention dans la RV, ces modèles doivent être étendus en 3-D et évalués lors de l'exécution des actions. Pour ce faire, ce chapitre explore l'applicabilité des modèles de DP du chapitre 4 pour prédire la performance des sélections. Ces prédictions de performance sont, à leur fois, utilisées pour prédire l'intention en les intégrant dans le cadre prédictif décrit dans le chapitre 3.

Pour généraliser les résultats des chapitres 3 et 4 , ce chapitre intègre un ensemble plus compréhensif de facteurs expérimentaux, ce qui inclut plus de niveaux de vélocité, d'angle initiale de la cible, et de nombre de sphères. De plus, ce chapitre est composé des tâches où les utilisateurs ne sont pas obligés d'attendre les cibles pour pouvoir les atteindre, ainsi que des tâches où les utilisateurs doivent choisir une cible spécifique parmi plusieurs autres.

\subsection{L'extension des modèles 2-D en 3-D}

Un avantage du modèle 2-D proposé dans le chapitre 4 est qu'il peut, théoriquement, être utilisé pour modéliser des tâches 3-D de sélection. Considérez la tâche générale de sélection 3-D présentée dans la figure 5.1. Même si la cible se déplace en 3-D, la vélocité (V) et la position initiale $(\mathbf{D})$ de la cible définissent un triangle rectangle aux côtés $D, D_{m}$ et $D_{s}$, qui constituent, avec $W$, les entrées du modèle de l'équation (4.20). 
En plus de l'altitude de la cible $(\theta)$, présente dans des tâches 2-D, les tâches 3-D requièrent un angle additionnel pour définir la position de la cible; dans ce travail on choisit un angle azimut $\alpha$, sur $z-x$. Dû à des problèmes de perception de profondeur propres aux affichages stéréoscopiques [Grossman and Balakrishnan 2004; Bowman et al. 2004], les cibles avec des $(\mathbf{V}, D)$ identiques, mais affichés à des profondeurs d'écran différentes, données par $(\theta, \alpha)$, peuvent être perçues avec des $D P$ différentes. Cet effet a été observé dans la littérature pour le TM requis afin d'acquérir des cibles statiques en 3-D [Teather and Stuerzlinger 2011].

De façon analogue aux tâches 2-D, les coordonnées angulaires initiales $(\alpha, \theta)$ pourraient être représentées avec des interceptes $a_{V \times \alpha \times \theta}$ différents. A nouveau, on formule l'hypothèse que, tant que la cible est placée dans le champs de vision de l'utilisateur, les $\alpha$ et $\theta$ initiaux auront un effet minimal sur le TM, puisque ces angles changent avec le mouvement de la cible.

De même, l'orientation de la cible ne peut plus être définie avec $\phi$. Comme montrée dans la figure 5.2, toute rotation $\gamma$ de $\mathbf{V}$ autour de $\mathbf{D}$ définit des triangles rectangles à dimensions $D_{s}$ et $D_{m}$ égales. Ayant des paramètres identiques, tous ces triangles sont considérés égaux dans les modèles 2-D du chapitre dernier ; cependant, si l'effet de $\gamma$ est large, le modèle 2-D de cibles en mouvement doit être adapté pour pouvoir être utilisé effectivement en 3-D.

\subsection{Difficulté subjective et performance}

Dans le chapitre 1 on affirme que les mesures prospectives et de performance sont liées, mais les premières peuvent complémenter ou même remplacer les dernières car elles ne dépendent pas de l'exécution des actions. Cependant, les mesures de performance, tel que le TM ou le pourcentage d'erreurs sont importantes pour évaluer les utilisateurs et les interfaces objectivement. De plus, la mesure de DP pour une tâche donnée requiert soit l'évaluation directe du participant (ce qui interrompt la tâche) ou l'utilisation de senseurs tel que l'EEg [Kourtis et al. 2012], qui peuvent être invasifs et ne sont pas disponibles dans toutes les configurations de Rv.

Se basant sur la corrélation entre la difficulté subjective (prospective et perçue) et le TM exploré dans la littérature, on propose que les formules de DP présentées dans le chapitre dernier peuvent aussi prédire le TM (cf. équation (5.5)).

La relation entre $D P$ et TM peut pourtant être différente pour des cibles en mouvement comparée aux cibles statiques. En particulier, dans le chapitre 4, on observe que le DP incrémente avec $V$ même pour les cibles qui se déplacent vers le curseur, mais dans ce cas-ci le TM devrait décrémenter avec $V$ tant que la cible n'a pas été manquée. Ceci impliquerait que les signes de quelques coefficients de régression dans les formules proposées pour DP peuvent avoir des signes contraires dans des formules équivalentes de TM. Par conséquent, ce chapitre investigue la relation entre les modèles de $D P$ pour la sélection de cibles en 
mouvement introduits lors du chapitre 4, et la performance des tâches 3-D de sélection de cibles en mouvement.

\subsection{Prédiction de l'intention de l'utilisateur dans de tâches dirigées}

Le chapitre 3 évalue la possibilité de prédire l'intention dans des tâches non-dirigées en utilisant l'ID, basée sur le postulat que les utilisateurs forment leurs intentions en minimisant leur effort prospectif, décrit par l'ID. Dans de tâches dirigées, comme celles étudiées dans ce chapitre, l'utilisateur doit choisir la cible indiquée quelque soit l'effort prospectif requis pour l'atteindre. Pourtant, le principe de minimiser l'effort prospectif peut toujours être valable au moment de choisir la trajectoire pour atteindre la cible, de façon similaire aux stratégies utilisées au choix de routes au quotidien [Christenfeld 1995; Bailenson et al. 2000].

Selon la stratégie du segment initiale [Christenfeld 1995; Bailenson et al. 2000], on a tendance a choisir des routes où les segments initiaux sont droits avec tournants se profilant vers la fin. Ces deux caractéristiques font partie du modèle pour la sélection 2-D de cibles en mouvement proposé dans la section 4.2.4. Ainsi, on évalue l'hypothèse que la cible voulue est celle avec le $\widehat{T M}$ minimal, comme prédit par l'équation (5.5), c'est ce qui est évalué dans ce chapitre. Comme la prédiction est basée seulement sur une caractéristique, on utilise une fonction de scoring basée sur celle proposée par Ortega [2013] (cf. équation (2.23)). A chaque frame $t, \widehat{T M}$ est calculé pour chacune des cibles en utilisant l'équation (5.5). Les cibles

sont subséquemment ordonnées de façon ascendante par $\widehat{T M}$, leur ordre étant indiqué par $i=0, \cdots, I-1$. Le score de chaque cible est calculé en suivant l'équation (5.6).

\subsection{Méthode}

\subsubsection{Participants}

Des participants d'Iowa State University furent recrutés à travers le pool de participants de recherche du département de psychologie (sONA), par le bouche à oreille et à travers la liste de courriels du département d'IHM.

Il y eut 33 participants, âgés de 18 à 39 ans $(M=23.48, M d n=21)$ dont dix femmes et un gaucher. La plupart de participants (31) étaient étudiants et 9 participants reportèrent avoir de l'expérience dans la Rv.

\subsubsection{Matériel}

L'expérience eut lieu au Virtual Reality Applications Center d'Iowa State University. Le code de la première expérience fut réutilisé et modifié pour implémenter cette expérience. L'application VR Jugglua fut déployée dans le C6, un Ev de type cave à six faces de $3.05 \mathrm{~m}^{3}$. Chacune des faces, excepté le mur arrière, fut projetée en utilisant du stéreo actif à $4096 \times 4096$ px. 
Les poses $(P, Q)$ de la tête et le wand du participant ont été suivis en utilisant le tracker ultrasonique. Enfin, l'Intersense Is-9oo permettait au participant de percevoir et intéragir avec le monde 3-D de façon adéquate.

\subsubsection{Procédure}

Sur le site de l'expérience, on demanda au participant de lire et accepter un formulaire de consentement pour participer à l'étude. Le consentement donné, on demanda au participant de répondre à une enquête Qualtrics concernant son background en jeux vidéo, RV, sports et ses données démographiques. Le participant devait compléter aussi un test de "prise de perspective/orientation spatiale » ("Perspective Taking/Spatial Orientation Test") [Hegarty and Waller 2004], et un questionnaire du mal en simulateur ("Simulator Sickness Questionnaire", ssQ) [Kennedy et al. 1993]. Subséquement, la vision du participant était évaluée en utilisant le « tableau interactif d'acuité visuelle » (Interactive Visual Activity Chart, IVAC) [Olitsky et al. 2006].

Après avoir completé le questionnaire, le participant fut demandé d'entrer dans le C6 et de se positionner sur une cible circulaire localisée au milieu de l'Ev $(0,0,0)$, faisant face au mur frontal. Si sa perception de l'environnent virtuel était adéquate, l'expérimentateur demanda alors au participant de choisir une position initiale pour le wand $\left(P_{w, \underline{0}}\right)$ qui soit confortable, en lui spécifiant de retourner sur cette position en début de chaque essai.

Dans chaque essai, un ensemble de sphères de tailles différentes, mais texturées identiquement, était initialement localisé en face du participant. Les mouvements démarraient avec des vitesses différentes, à partir du moment où le wand du participant rentrait dans une sphère verte translucide $\left(s p h_{\underline{0}}\right)$, de rayon $0.05 \mathrm{~m}$, centrée sur $P_{w, \underline{0}}$.

On requera au participant de laisser le wand dans $P_{w, \underline{0}}$ jusqu'à ce que la sphère but soit indiquée. Dans le cas contraire, la $P_{w, \underline{0}}$ devenait rouge. Une fois la sphère but indiquée, $P_{w, \underline{0}}$ disparaissait et le participant devait étendre son bras pour atteindre la sphère but. Chaque sphère, but ou non, disparaissait lors du contact avec le wand. L'essai ne finissait qu'au moment où la sphère but était touchée, ou quand sa distance avec la tête de l'utilisateur était supérieure à $2 \mathrm{~m}$, au quel cas elle était considérée inatteignable.

A chaque frame, le temps écoulé, les poses de la tête du participant $\left(P_{h}, Q_{h}\right)$ et du wand $\left(P_{w}, Q_{w}\right)$, chacune des positions des sphères $\left(P_{i}\right)$, et les collisions wand-sphère possibles étaient enregistrés. La configuration expérimentale est montrée dans la figure 5.3.

Une fois tous les essais complétés, on demanda au participant de sortir du C6 et de remplir un questionnaire post-expérience, ce qui incluait le sso. La longueur totale de l'étude était de moins de 60 minutes. 


\subsubsection{Protocole}

Un protocole factoriel, intra-sujets à été utilisé, avec deux blocs d'essais. Dans le premier bloc il y avait une sphère par essai. ${ }^{1}$ La cible était placée sur un des coins d'un tétraèdre imaginaire aux cotés d'1 $\mathrm{m}$, un coin placé à $P_{w, \underline{0}}$ et les trois autres placés en face du participant. Les facteurs étaient la position de la cible, $P \in\{$ haut,bas-gauche,bas-droite $\}$, son rayon, $r \in\{0.1,0.2\} \mathrm{m}$, sa vitesse, $V \in\{0.5,1,1.5\} \mathrm{m} / \mathrm{s}$, et sa direction initiale de mouvement, $\phi \in\{15,30,45\}^{\circ}$. Etant donné qu'il y avait déjà 54 conditions, la valeur initiale de l'angle $\gamma$ était choisie aléatoirement entre $180^{\circ}$ et $360^{\circ}$. Il y avait un essai par condition, pour un total de 1,782 essais.

Dans le deuxième bloc il avait trois sphères par essai. Chaque sphère était placée sur un des trois coins frontaux d'un tétraèdre avec la même configuration que celui décrit dans le paragraphe précédant. Les facteurs étaient la position de la cible $P \in\{$ haut, bas-gauche,bas-droite $\}$, son rayon, $r \in\{0.1,0.2\} \mathrm{m}$, sa vitesse, $V \in\{0.5,1,1.5\} \mathrm{m} / \mathrm{s}$, et sa direction initiale de mouvement, $\phi \in\{15,30,45\}^{\circ}$. La valeur initiale de l'angle $\gamma$ était choisie aléatoirement entre $180^{\circ}$ et $360^{\circ}$, et les conditions $(P, r, V, \gamma)$ des deux sphères restantes étaient aussi choisies aléatoirement parmi les valeurs expérimentales possibles. Il avait un essai par chacune des 54 conditions, pour un total de 1,782 essais.

Dans le dernier bloc il avait six sphères par essai. Chaque sphère était placée sur un des coins frontaux de deux tétraèdres imaginaires, un avec la configuration décrite précédemment et l'autre ayant des coins identiques au premier, mais intervertis verticalement (cf. l'image centrale de la figure 5.3). Les facteurs étaient la position de la cible $P \in\{$ haut, haut-gauche, bas-gauche, bas, bas-droite, haut-droite $\}$, son rayon, $r \in\{0.1,0.2\} \mathrm{m}$, sa vitesse, $V \in\{0.5,1,1.5\} \mathrm{m} / \mathrm{s}$, et sa direction initiale de mouvement, $\phi \in\{15,30,45\}^{\circ}$. La valeur initiale de l'angle $\gamma$ était choisie aléatoirement entre $180^{\circ}$ et $360^{\circ}$, et les conditions $(P, r, V, \gamma)$ des deux sphères restantes étaient aussi choisies aléatoirement parmi les valeurs expérimentales possibles. Il avait un essai par chacune des 108 conditions, pour un total de 3,564 essais.

Dans tous les essais de toutes les conditions, les positions initiales des sphères étaient $P_{i}-\mathbf{V}_{i} * 1 \mathrm{~s}$. La sphère but était marquée après avoir dépassé sa position $P$ dans le tétraèdre, i.e., après approximativement $1 \mathrm{~s}$, quand elle était à environ $D=1 \mathrm{~m} \cdot{ }^{2}$ Le critère pour marquer la sphère but était $D<1 \mathrm{~m}$. Notez qu'au moment où la sphère but était marquée, toutes les sphères étaient approximativement à leur position $P_{i}$ dans le tétraèdre, à la même distance de $P_{w, \underline{0}}$. La raison fut de donner aux participants du temps pour percevoir le mouvement des différentes sphères et pour rendre les arrangements des sphères apparemment aléatoires.

\footnotetext{
${ }^{1}$ On demanda toutefois, au participant de rester dans $P_{w, \underline{0}}$ avant que cette sphère devienne marquée.

${ }^{2}$ Ces valeurs sont approximatives dû au frame rate variable.
} 


\subsection{Discussion}

Les résultats sont synthétisés par rapport aux hypothèses formulées, chacune suivie par une discussion.

\subsubsection{Performance de la sélection 3-D de cibles en mouvement}

H1. Le modèle en trois parties avec des coefficients par-vitesse et l'intercepte par vélocité, décrit dans l'équation (5.5), modélise de façon précise le temps de mouvement des tâches tridimensionnelles de sélection de cibles en mouvement. Validée.

Le modèle en trois parties de sélection de cibles de l'équation (5.5) s'est bien ajusté aux donnés, avec des valeurs crédibles de $R^{2} \in[0.89,0.96]$ (c.f., tableau 5.1). Les résidus, montrés dans la figure 5.10, indiquent une variance plus large pour $V=0.5$, comparée à $V \in 1.0,1.5$, mais étant donné qu'il n'y a que deux données aberrantes visibles, on n'amène plus de modifications au modèle. Etant donné qu'un modèle très similaire fut utilisé pour prédire $D P$, ces résultats apportent plus de support à la relation entre DP et TM.

H1.1. L'azimut $\alpha$, l'altitude $\theta$ et l'angle $\gamma$, non-modélisés dans l'équation (5.5), ont un effet moindre sur le TM. Partiellement validée.

Les angles $\alpha$ et $\theta$ ont été variés simultanément en tant que positions des sphères, ce qui empêcha d'évaluer leurs effets séparément. La figure 5.6 suggère que les cibles localisées en haut par rapport à $s p h_{\underline{0}}$ eurent un TM que les cibles localisées en dessous de $s p h_{\underline{0}}$, mais cet effet n'est pas visible dans le graphique des résidus (cf. figure 5.10).

Finalement, comme montré dans la figure 5.7, le TM resta très similaire à travers le rang des angles $\gamma$. Ceci suggère un effet moindre de $\gamma$ sur le TM.

H1.1. Dans l'équation (5.5) les coefficients $a_{V}, b_{V}, c_{V}$ et $d_{V}$ varient linéairement avec $V$. Validée.

Les résultats suggèrent que ces coefficients décrementent linéairement avec l'increment de $V$. En utilisant les intervalles de crédibilité du tableau 5.3, on peut prédire le TM en utilisant des $V$ différents, tout en propageant l'incertitude sur ces estimés.

\subsubsection{Prédiction d'intention dans des tâches 3-D de sélection dirigée de cibles en mouvement}

H2. A un instant donné, le temps minimal de mouvement estimé par l'équation (5.5) peut être utilisé pour prédire la cible voulue dans une tâche 3-D de sélection dirigée de cibles en mouvement. Partiellement validée. 
Même s'il a été possible de prédire la sphère voulue en utilisant le TM estimé via la fonction de scoring $\widehat{T S} \operatorname{core}_{i}(t)$ avec, la plupart du temps, une précision meilleure que la chance, la précision résultante fut très faible. Dans certains cas du bloc de six sphères, la précision fut même plus basse que la chance (cf. figure 5.14).

Notez, pourtant, que les estimations des paramètres utilisées pour calculer $\widehat{T M}$ furent obtenues du bloc d'une sphère. Contrairement aux blocs de trois et six sphères, ce bloc incluait un rang différent d'entrées $D_{s}$ et $D_{m}$, avait $T R=0$ et n'était affecté ni par des problèmes d'occlusion ni par de problèmes de clutter. Même si la loi de Hick-Hyman permet de modeliser $T R=\log _{2}(I+1)$, où $I$ est le nombre de cibles dans la scène, avec l'increment du $T R$, le $D_{m}$ est réduit par $T R \times V$, et la position initiale du wand peut changer, résultant possiblement dans un $D_{s}$ différent. Par conséquent, l'intégration de la loi de Hick-Hyman dans le modèle actuel n'est pas évidente.

Une façon plus viable d'améliorer les prédictions de $\widehat{T M}$ dans des blocs à plusieurs cibles, améliorant aussi potentiellement la précision de $\widehat{T} S \operatorname{core}_{i}(t)$, serait d'inclure des variations par-sujet dans les paramètres de l'équation (5.5). En effet, les estimations des paramètres du tableau 5.1 sont données pour le temps moyen TM par condition et non pour chaque essai.

H2.1. La précision des prédictions basées sur le temps estimé de mouvement sera meilleure que celle des prédictions basées sur la distance wand-cible. Partiellement validée.

La fonction de scoring $\widehat{T} S \operatorname{score}_{i}(t)$ donna des prédictions plus précises que $d \operatorname{Score}_{i}(t)$ juste pour des essais de 3 et 6 sphères avant $0.5 T$ et $0.4 T$, respectivement. Dans les temps postérieurs, la fonction $d \operatorname{Score}_{i}(t)$ était toujours plus précise. Comme suggéré précédemment, cette inversion dans la précision des prédictions peut indiquer une transition vers une phase balistique de mouvement au fur à mesure que l'essaie avance, dans laquelle $D \simeq D_{m}$ a plus d'influence sur $T$. 


\section{Chapitre 6}

\section{Conclusion}

Cette thèse offre une investigation profonde et empirique sur la sélection de cibles en mouvement, une tâche qui est de plus en plus commune et pourtant peu étudiée dans l'interaction homme-machine. En particulier, des modèles prédictifs furent développés selon trois étapes de l'action la sélection une cible en mouvement : l'intention, la difficulté prospective et la performance.

Les modèles de prédiction d'intention furent developpés en utilisant des arbres de décision et des fonctions de scoring avec des caractéristiques spécifiques à des tâches de sélection non-dirigée (i.e., des tâches où les utilisateurs sont libres de choisir un objet parmi plusieurs autres), et des caractéristiques généralisables qui peuvent aussi être utilisées dans des tâches de sélection dirigée (i.e., les tâches expérimentales les plus communes où l'on dit aux utilisateurs de choisir une cible en particulier). Dans le chapitre 3, on trouva que la taille des cibles $(W)$ était la caractéristique la plus importante pour prédire l'intention dans la sélection non-dirigée, avec une précision de $\sim 71 \%$ pour deux cibles aux vélocités égales et aux positions initiales différentes. Cela était contraire à une expectative selon laquelle l'ID, mesuré au début de chaque essai, aurait été la caractéristique la plus prédictive dans ce type de tâche. En ce qui concerne les caractéristiques généralisables dans la sélection non-dirigée, les résultats du chapitre 3 indiquent qu'une combinaison du regard relatif tête-cible $(\Delta d o t)$ et la distance relative wand-cible $(\Delta D)$, moyennés dans une fenêtre de temps de 1-1.5s, étaient prédictifs de l'intention de sélection avec une précision de $\sim 72 \%$. La combinaison des caractéristiques spécifiques et généralisables dans la sélection non-dirigée ( $\sim 78 \%)$. Enfin, dans le chapitre 5 , les fonctions de scoring basés sur le temps de mouvement estimé $(\widehat{T M})$ et la distance curseur-cible, donnèrent des meilleures précisions que la chance, mais assez faibles, en prédisant la cible voulue dans une tâche dirigée de sélection avec 3 et 6 sphères.

Des modèles de difficulté prospective $(D P)$ furent développés et évalués dans des tâches 1-D et 2-D de sélection de cibles en mouvement, dans le chapitre 4. Ces modèles décrivent le mouvement requis pour atteindre une cible en mouvement en trois parties : deux mouvements balistiques, un pour aligner le curseur avec l'axe de mouvement de la cible et un autre aligné avec l'axe de mouvement de la cible, et un mouvement de rapprochement en ligne avec l'axe 
de mouvement de la cible. Les mouvements balistiques furent représentés avec les racines carrées des distances, tandis que le mouvement de rapprochement fut représenté avec l'ID de Fitts. Dans l'ensemble, la $D P$ incrémentait avec la vitesse $(V), 1^{\prime} I D$, et la distance curseur-cible, et diminuait avec la distance curseur-cible sur l'axe de mouvement de la cible. Contrairement aux expectatives, la vitesse eu une influence moindre sur le mouvement balistique requis pour aligner le curseur avec l'axe de mouvement de la cible, et le mouvement de rapprochement de la cible sur l'axe de mouvement de la cible.

La prédiction de la performance fut explorée dans le chapitre 5 en évaluant l'usage du modèle 2-D présenté dans le chapitre 4 en tant que prédicteur du temps moyen de mouvement $(\overline{T M})$ dans la sélection 3-D de cibles en mouvement. Le modèle en trois parties s'est bien ajusté aux données, mais contrairement au modèle de $D P$, les coefficients liés à des mouvements balistiques et de rapprochement ont décrémenté quand la vitesse incrémentait. Cette différence était espérée, étant donné que les cibles les plus rapides requièrent un temps de mouvement plus court pour pouvoir être sélectionnées de façon satisfaisante. 


\section{ÉTUDE DE LA PRÉDICTION DE L'ACTION DE L'UTILISATEUR DANS UNE TÂCHE DE SÉLECTION DE CIBLES EN MOUVEMENT}

Résumé : La sélection de cibles en mouvement est une tâche courante et complexe dans l'interaction homme-machine (IHM) en général et en particulier dans le domaine de la réalité virtuelle (Rv). La prédiction de l'action est une solution intégrale pour aborder les problèmes liés à l'interaction. Cependant, les techniques actuelles de prédiction sont basées sur le suivi continu des actions de l'utilisateur sans prendre en compte la possibilité que les actions d'atteinte d'une cible puissent avoir une composante importante préprogrammée-cette théorie est appelé la théorie du contrôle préprogrammé.

En se basant sur la théorie du contrôle préprogrammé, cette thèse explore la possibilité de prédire les actions, avant leur exécution, de sélection d'objets en mouvement. Plus spécifiquement, trois niveaux de prédiction d'action sont étudiés : 1) la performance des actions, mesurée par le temps de mouvement (TM) nécessaire pour atteindre une cible, 2) la difficulté prospective $(D P)$, qui représente la difficulté subjective de la tâche estimée avant son exécution, 3) l'intention de l'utilisateur, qui indique la cible visée par l'utilisateur.

Dans le cadre de cette thèse, des modèles de prédiction d'intention sont développés à l'aide des arbres de décision ainsi que des fonctions de classement-ces modèles sont évalués dans deux expériences en RV. Des modèles 1-D et 2-D de DP pour des cibles en mouvement basés sur la loi de Fitts sont développés et évalués dans une expérience en ligne. Enfin, des modèles de $T M$ avec les mêmes caractéristiques structurelles des modèles de $D P$ sont évaluées dans une expérience 3-D en RV.

Mots clés : Sélection de cibles en mouvement, intention, difficulté prospective, performance, loi de Fitts, interaction homme-machine, réalité virtuelle.

\section{Prediction of USER ACtion In MOVING-TARget Selection tasks}

Aвstract: Selection of moving targets is a common, yet complex task in human-computer interaction ( $\mathrm{HCI}$ ), and more specifically in virtual reality (VR). Action prediction has proven to be the most comprehensive enhancement to address moving-target selection challenges. Current predictive techniques, however, heavily rely on continuous tracking of user actions, without considering the possibility that target-reaching actions may have a dominant pre-programmed component-this theory is known as the pre-programmed control theory.

Thus, based on the pre-programmed control theory, this research explores the possibility of predicting moving-target selection prior to action execution. Specifically, three levels of action prediction are investigated: 1) action performance measured as the movement time $(M T)$ required to reach a target, 2) prospective difficulty $(P D)$, i.e., subjective assessments made prior to action execution; and 3) intention, i.e., the target that the user plans to reach.

In this dissertation, intention prediction models are developed using decision trees and scoring functions-these models are evaluated in two vR studies. PD models for 1-D, and 2-D moving-target selection tasks are developed based on Fitts' Law, and evaluated in an online experiment. Finally, MT models with the same structural form of the aforementioned $P D$ models are evaluated in a 3-D moving-target selection experiment deployed in VR.

Keywords: Moving-target selection, user intention, prospective difficulty, performance, Fitts' Law, human-computer interaction, virtual reality. 